\title{
ELECTRONUCLEAR DIVISION
} ANNUAL PROGRESS REPORT

FOR PERIOD ENDING DECEMBER 31, 1962

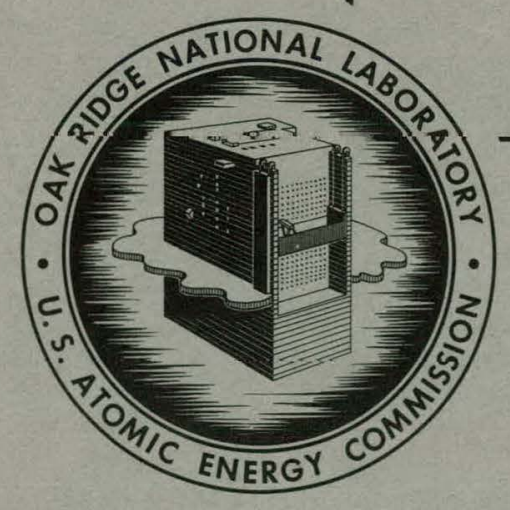

OAK RIDGE NATIONAL LABORATORY

$$
\text { operated by }
$$

UNION CARBIDE CORPORATION

for the

U.S. ATOMIC ENERGY COMMISSION 


\section{DISCLAIMER}

This report was prepared as an account of work sponsored by an agency of the United States Government. Neither the United States Government nor any agency Thereof, nor any of their employees, makes any warranty, express or implied, or assumes any legal liability or responsibility for the accuracy, completeness, or usefulness of any information, apparatus, product, or process disclosed, or represents that its use would not infringe privately owned rights. Reference herein to any specific commercial product, process, or service by trade name, trademark, manufacturer, or otherwise does not necessarily constitute or imply its endorsement, recommendation, or favoring by the United States Government or any agency thereof. The views and opinions of authors expressed herein do not necessarily state or reflect those of the United States Government or any agency thereof. 


\section{DISCLAIMER}

Portions of this document may be illegible in electronic image products. Images are produced from the best available original document. 


\section{Printed in USA. Price: $\$ 2.25$ Available from the}

Office of Technical Services

U. S. Department of Commerce

Washington 25, D. C.

\section{LEGAL NOTICE}

This report was prepared as an account of Government sponsored work. Neither the United States, nor the Commission, nor any person acting on behalf of the Commission:

A. Makes any warranty or representation, expressed or implied, with respect to the accuracy, completeness, or usefulness of the information contained in this report, or that the use of any information, apparatus, method, or process disclosed in this report may not infringe privately owned rights; or

B. Assumes any liabilities with respect to the use of, or for damages resulting from the use of any information, apparatus, method, or process disclosed in this report.

As used in the above, "person acting on behalf of the Commission" includes any employee or contractor of the Cammission, or employee of such contractor, to the extent that such employee or contractor of the Commission, or employee of such contractor prepares, disseminates, or provides access to, any information pursuant to his employment or contract with the Commission, or his employment with such contractor. 
ORNL-3432

UC.34 - Physics

TID.4500 (19th ed., Rev.)

Contract No. W-7405-eng-26

\section{ELECTRONUCLEAR DIVISION}

\section{ANNUAL PROGRESS REPORT}

For Period Ending December 31, 1962

Robert S. Livingston, Director

Alexander Zucker, Associate Director

DATE ISSUED

MAY 241963

OAK RIDGE NATIONAL LABORATORY

Oak Ridge, Tennessee operated by UNION CARBIDE CORPORATION for the

U.S. ATOMIC ENERGY COMMISSION 


\section{THIS PAGE}

\section{WAS INTENTIONALLY \\ LEFT BLANK}




\section{Summary}

Heavy-ion reactions in the low- $Z$ region were investigated with $27-\mathrm{Mev}$ nitrogen ions from the 73-Inch Cyclotron and with 30-Mev oxygen ions from the Tandem Van de Graaff. Experiments included studies of angular distributions and excitation functions for transfer reactions, compound-nucleus reactions, effects of angular momentum on the density of nuclear states, and so-called Ericson fluctuations in the compound nucleus $\mathrm{Si}^{28}$.

Nuclear reactions induced with $22-\mathrm{Mev}$ protons in the 86-Inch Cyclotron were used in studies of the pickup-reaction mechanism, shell-model studies from pickup reactions, scattering and the optical model, and level schemes in highly deformed nuclei.

In theoretical investigations of the mechanisms of nuclear reactions and related nuclear-structure information, the elastic scattering of complex particles was analyzed via the optical model; the applicability of the distorted-wave method to stripping reactions was investigated, and the inelastic scattering of carbon ions from carbon was examined.

Experimental studies with the eight-sector electron model, Analogue II, led to very successful deflection of the beam in November 1962, a significant advance in accelerator technology. This investigation, still incomplete, eases one of the major uncertainties associated with the proposed $\mathrm{Mc}^{2}$ cyclotron. The radioactivity resulting from the residual undeflected beam being dumped onto the structure of the cyclotron can now be estimated much more reliably.

Successful test operation of the Oak Ridge Isochronous Cyclotron (ORIC) at full radius in March 1962 verified the design of the azimuthally varying magnetic field, which is expected to provide for fixed-frequency operation for proton energies up to $75 \mathrm{Mev}$. During these test operations at $8 \mathrm{Mev}$, the properties of the beam at full radius were explored, and the beam was brought through an electrostatic deflector channel. The machine was then shut down for the installation of the full-power if system and the beam-extraction somponents.

Investigation of basic problems connected with the design of large magnets with $\mathrm{Nb}-\mathrm{Zr}$ superconductors was continued. It was found that the use of a copper secondary, bifilar wound with the superconductor, and the use of epoxy casting, to rigidly confine the windings, markedly increase the current capacity of the magnet and eliminate the damage usually encountered when the superconductor goes normal. 


\section{THIS PAGE \\ WAS INTENTIONALLY \\ LEFT BLANK}




\section{Contents}

SUMMARY

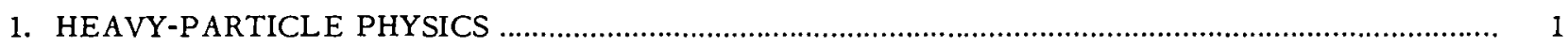

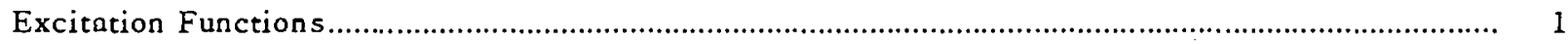

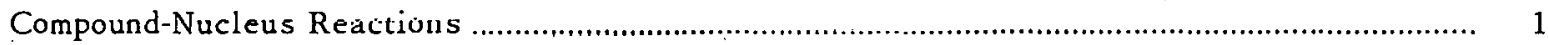

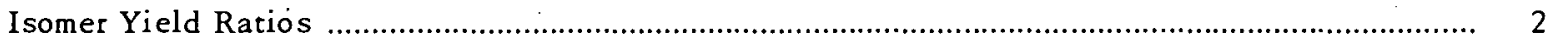

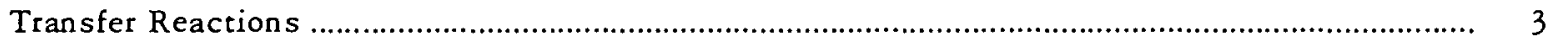

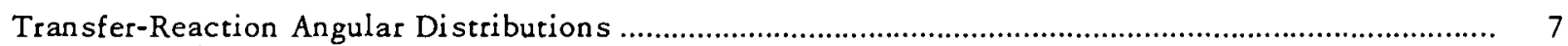

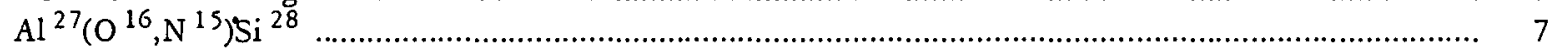

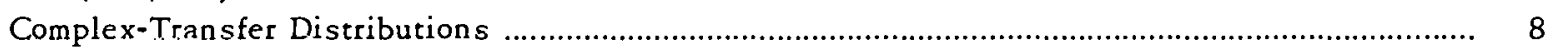

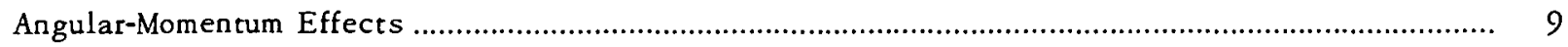

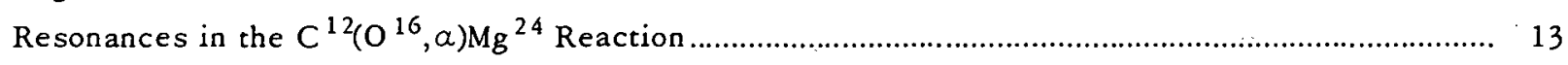

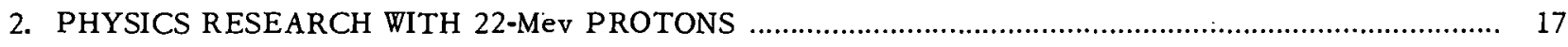

Studies of the Pickup-Reaction Mechanism ............................................................................... 17

Shell-Model Studies Using Pickup Reactions ................................................................................ 18

Scattering and the Optical Model ........................................................................................... 21

Systemaric Properties of Nuclear Levels in Deformed Odd-A Nuclei ............................................... 22

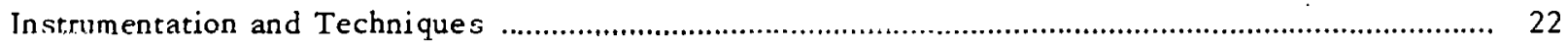

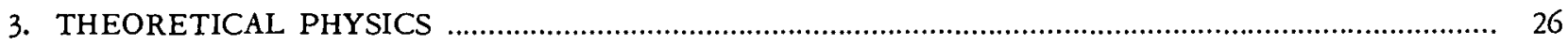

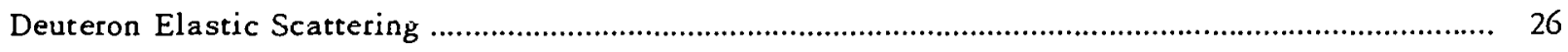

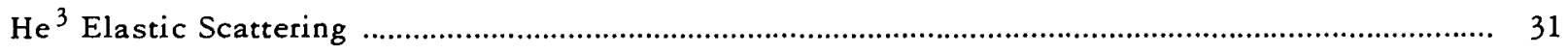

Elastic and Inelastic Scattcring of Carbon Ions from Carbon .......................................................... 32

Finite-Range Correction to Distorted-Wave Approximation ...............................................................

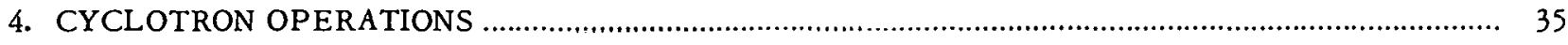

Operation of the Oak Ridge Isochronous Cyclotron ........................................................................ 3.5

Operation of the ORNL 63-Inch Cyclotron ....................................................................................... 35

Operation of the ORNL 86-Inch Cyclotron ..........................................................................................

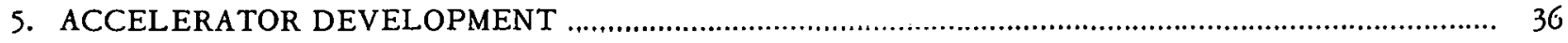

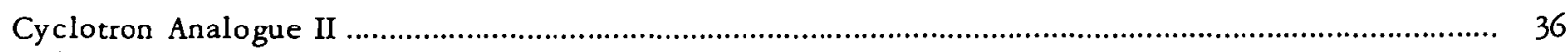

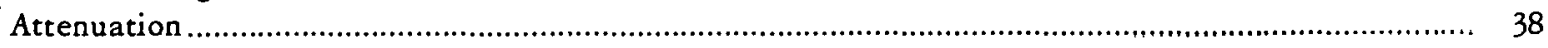

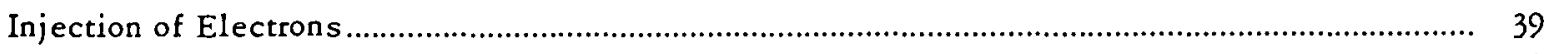

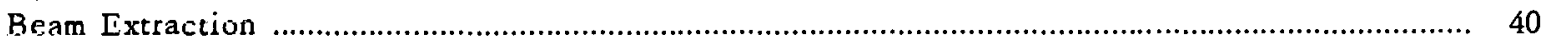




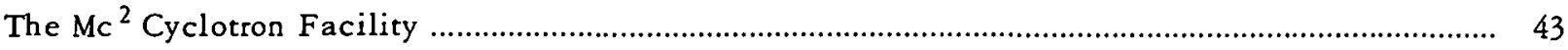

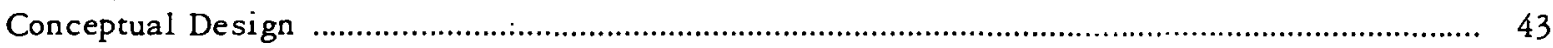

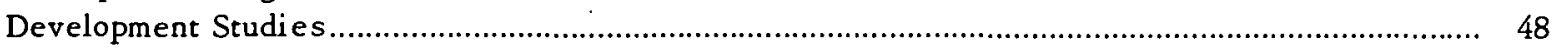

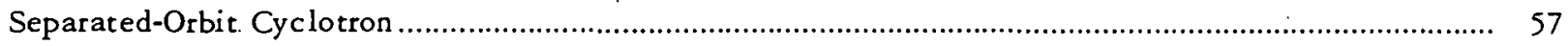

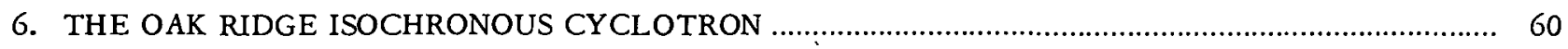

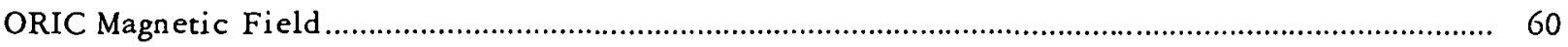

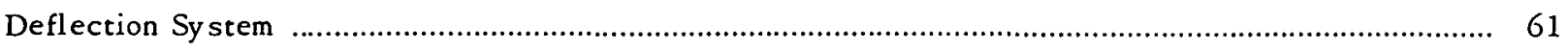

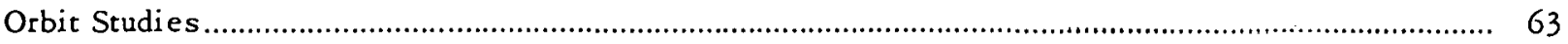

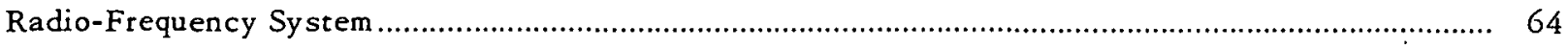

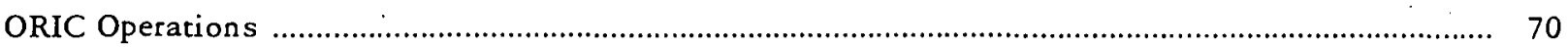

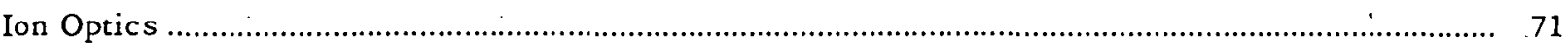

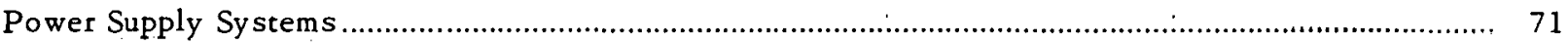

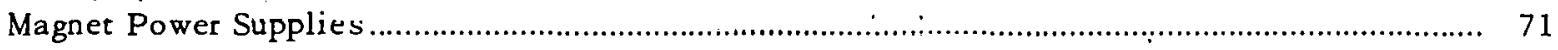

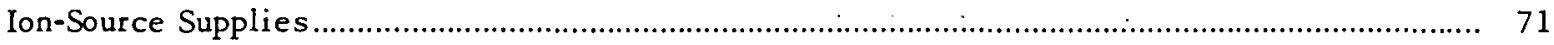

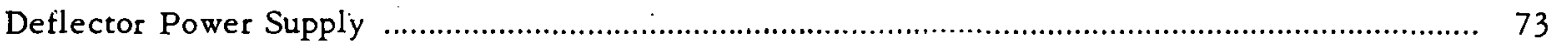

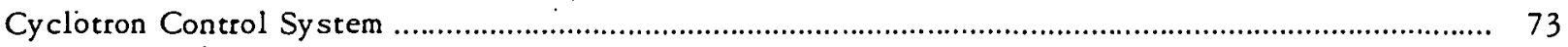

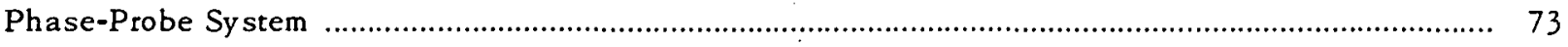

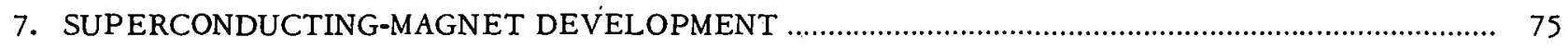

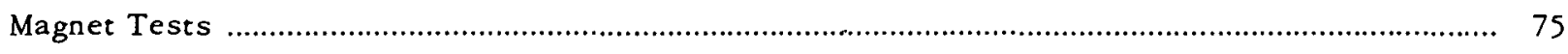

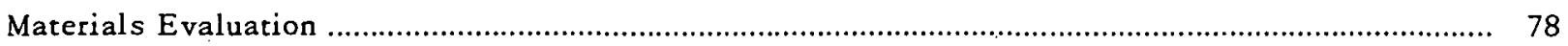

PAPERS PRESENTED AT SCIENTIFIC AND TECHNICAL MEETINGS ........................................... 80

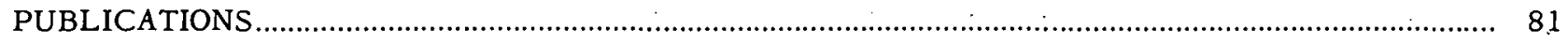

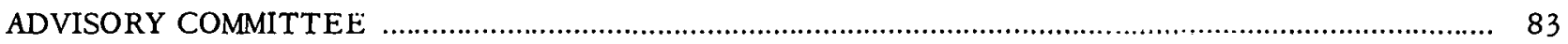

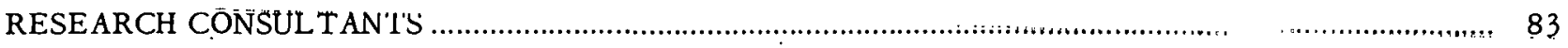

MISCELLANEOUS ACTIVITIES OF THE ELECIRONUCLEAR DIVISION …........................................ 84 


\section{Heavy-Particle Physics}

\author{
M. L. Halbert \\ H. F. Bowsher ${ }^{1}$ \\ F. Newman \\ F. E. Durham ${ }^{2}$
}

' 1 'he study of heavy-ion reactions in the low- $Z$ region included experiments on transfer-reaction excitation functions and angular distributions, compound-nucleus reactions, the effect of angular momentum on the density of nuclear states, and the investigation of so-called Ericson fluctuations in the compound nucleus $\mathrm{Si}^{28}$. In addition, new scattering chambers and other equipment were designed and built for use on ORIC. During six weeks of running time on the tandem accelerator, an $\mathrm{O}^{16}$ beam was used for various experiments. The ORNL 63-Inch Cyclotron was reactivated occasionally for $\mathrm{N}^{14}$ bombardments.

\section{EXCITATION FUNCTIONS}

During the past year an extensive program was carried out in determining excitation functions for reactions induced by $27.5-\mathrm{Mev} \mathrm{N}^{14}$ ions. The excitation functions were determined by the usual method of bombarding thick targets at various energies. The resultant products were identified, and their decay was followed by the use of a calibrated NaI crystal spectrometer. In most instances no chemical separations were necessary, since the radioactive products could be identified unambiguously simply by means of their half-lives and gamma radiations. Twenty-two excitation functions were obtained in three rather separate investiga-

\footnotetext{
${ }^{1}$ Research participant, University of Tennessee.

${ }^{2}$ Research participant, Tulane University.

${ }^{3}$ Graduate student, University of Tennessee.

${ }^{4}$ Physics Division (collaborated on resonances in $\left.\mathrm{C}^{12}+\mathrm{O}^{16}\right)$.
}
K. S. Toth
M. E. Wetzen ${ }^{3}$
A. Zucker
C. D. Moak

tions: (1) the determination of cross sections for reactions proceeding through a compound-nucleus stage; (2) the investigations of isomeric yield ratios for $\mathrm{Sc}^{44 m}$ and $\mathrm{Sc}^{44 \mathrm{~g}}$ as produced by the $\mathrm{N}^{14}$ bombardment of $\mathrm{P}^{31}, \mathrm{~S}^{32}$, and $\mathrm{Cl}^{35}$; and (3) the study of single-nucleon transfer-reaction cross sections.

\section{Compound-Nucleus Reactions}

The production of the following nuclides from various target nuclei was investigated: $\mathrm{Ne}^{23}$ from $\mathrm{B}^{11} ; \mathrm{P}^{30}$ from $\mathrm{F}^{19} ; \mathrm{K}^{38}$ from $\mathrm{Al}^{27} ; \mathrm{Cl}^{34 m}$ and $\mathrm{K}^{38}$ from silicon; $\mathrm{Sc}^{43}, \mathrm{Sc}^{44 m}$, and $\mathrm{Sc}^{44 g}$ from $\mathrm{P}^{31}$; $\mathrm{Sc}^{43}, \mathrm{Sc}^{44 m}$, and $\mathrm{Sc}^{44 \mathrm{~g}}$ from $\mathrm{S}^{32} ; \mathrm{Sc}^{43}, \mathrm{Sc}^{44 m}$, $\mathrm{Sc}^{44} \mathrm{~g}^{2} \mathrm{Ti}^{45^{\prime}}$, and $\mathrm{V}^{47}$ from $\mathrm{Cl}^{35}$; and $\mathrm{Cr}^{49}$ from $\mathrm{Cl}^{37}$. Owing to the lack of space, only the chlorine results are shown in Fig. 1.1, particularly since the ensuing discussion is limited to reactions resulting from the bombardment of $\mathrm{Cl}^{35}$.

Cross sections were measured for the production of four isotopes from the $\mathrm{N}^{14}$ bombardment of $\mathrm{Cl}^{35}$, that is, $\mathrm{V}^{47}, \mathrm{Ti}^{45}, \mathrm{Sc}^{44}$, and $\mathrm{Sc}^{43}$. These experi mental data from final isotopes resulting from one compound nucleus $\left(\mathrm{Cr}^{49}\right)$ can be compared with the predictions of the statistical theory. The theory of the emission of particles from an excited compound nucleus is originally due to Weisskopf. ${ }^{5}$ Recently, in a series of papers Dostrovsky et al. ${ }^{6}$

\footnotetext{
${ }^{5}$ V. F. Weisskopf, Pbys. Rev. 52, 295 (1937).

${ }^{6}$ I. Dostrovsky, Z. Fraenkel, and G. Friedlander, Phys. Rev. 116, 683 (1959); I. Dostrovsky, Z. Fraenkel, and L. Winsberg, Pbys. Rev. 118, 781 (1960); and I. Dostrovsky, Z. 'Fraenkel, and P.' Rabinowitz, Phys. Rev. 118, 791 (1960):
} 


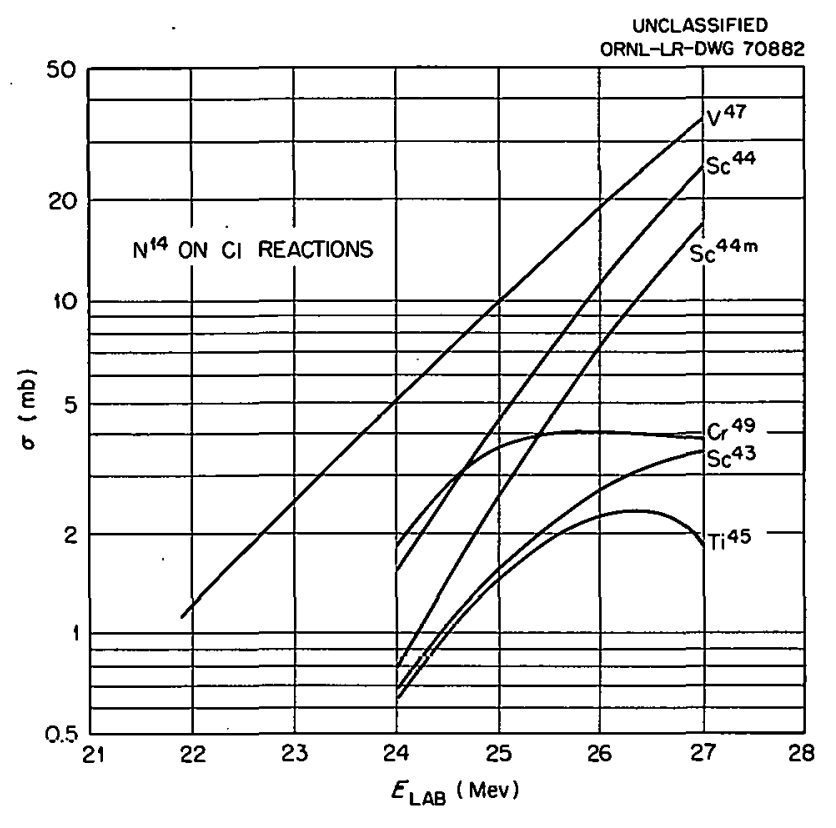

Fig. 1.1. Excitation Functions for Reactions Induced by $\mathrm{N}^{14}$ lons on Chlorine.

described a Monte Carlo calculation based on this theory. Their calculations incorporate an analytic form for the inverse cross section chosen to approximate square-well potential cross sections. The form of the level density does not contain a $1 / E^{2}$ term; neither gamma-ray de-excitations nor angular momentum effects are considered. In addition, no attempt is made to include the effects of nuclear clusters on the initial stages of the cascade. An IBM 7090 program essentially duplicating this approach has become available at ORNL. This program was utilized to determine the applicability of the Monte Carlo calculations in their present form for the compound system of $\mathrm{N}^{14}$ and $\mathrm{Cl}^{35}$.

$A$ search on the level-density parameter $a$ was made to determine the value best fitting the data, for an incident energy of $27 \mathrm{Mev}$. It was found that the predicted ratios did not agree with the experimental ratios for any value of $a$. The best agreement was found, however, when $a$ was equal to 1.70 . The experimental and theoretical crosssection ratios are compared in Table 1.1.

The reason for the poor agreement and the necessity for a low value of $a$ arises from the fact that the Monte Carlo calculations predict very few emitted alphas in comparison with neutrons and protons. The experimental data, on the other hand, clearly show that alpha emission competes successfully with neutron and proton emission. Thus, the dearth of predicted alpha emission is responsible for the lack of agreement with experiment. In addition, it is found that the number of emitted alphas has a maximum for $a \sim 1.70$. This is the reason why it is necessary to select a low value to obtain the best fit with experiment.

The value of $a$ best reproducing the data, excluding the regions near magic numbers, is usually $a$ $\sim A / 10$. One of several possible explanations for the appearance of a small value of the level-density parameter and the poor agreement between theoretical and experimental cross-section ratios is the absence of an $E^{-2}$ term in the level-density exprescion.

The Monte Carlo calculations were modified to include this type of level density, and a search on $a$ was used to determine the best value. As before, the overall agreement is very poor, with the best fit occurring at $a \sim 5.0$. The values obtained are also shown in Table 1.1.

One additional modification was made in the original program. This was the replacement of the analytic form of the inverse cross sections by corrcsponding cross sections calculated from opticalmodel transmission coefficients. It was found that this increased the predicted number of nuclei formed by alpha emission by $\sim 15 \%$. This effect, however, is insufficient to account for the discrepancy between the experimental data and the statistical-model calculations.

\section{Isomer Yield Ratios}

The isomer yield ratio $\mathrm{Sc}^{44 m} / \mathrm{Sc}^{44 g}$ for the production of $\mathrm{Sc}^{44}$ from the reactions $\mathrm{P}^{31}\left(\mathrm{~N}^{14}, p\right) \mathrm{Sc}^{44}$, $\mathrm{S}^{32}\left(\mathrm{~N}^{14}, 2 p\right) \mathrm{Sc}^{44}$, and $\mathrm{Cl}^{35}\left(\mathrm{~N}^{14}, \alpha p\right) \mathrm{Sc}^{44}$ was determined. These ratios are shown in Fig. 1.2, plotted as a function of $E_{\text {c.m. }}$. The steady, sharp increase of the ratio with bombarding energy for $P^{31}$ and $\mathrm{S}^{32}$ is consistent with the $\mathrm{Sc}^{44}$ being produced by means of a compound-nucleus mechanism. As the energy and the angular momentum brought into the compound nucleus are increased, the probability of the residual nucleus being left in a state of higher angular momentum also increases and the ratio is thus expected to increase. The $\mathrm{Cl}^{35}$ ratio, on the other hand, rises gently and then levels off at $\sim 18.6 \mathrm{Mev}$. This behavior can be explained in two ways. First, the loss from the compound system $\left(\mathrm{Cr}^{49}\right)$ of a large amount of angular momentum, 
Table 1.1. Experimental and Theoretical Cross-Section Ratios ${ }^{a}$ for Reactions Resulting from the Bombardment of $\mathrm{Cl}^{35}$ by $\mathrm{N}^{14}$

\begin{tabular}{|c|c|c|c|}
\hline Products & Experimental & $\begin{array}{c}\text { Theoretical Without } 1 / E^{2} \\
(a=1.7)\end{array}$ & $\begin{array}{l}\text { Theoretical With } 1 / E^{2} \\
(a=5.0)\end{array}$ \\
\hline$\frac{\mathrm{Sc}^{44}}{\mathrm{~V}^{47}} \frac{(a p)}{(p n)}$ & $1.18 \pm 0.28$ & $0.27 \pm 0.01$ & $0.26 \pm 0.05$ \\
\hline$\frac{\mathrm{Sc}^{44}}{\mathrm{Ti}^{45}} \frac{(\alpha p)}{(\alpha)}$ & $20.0 \pm 4.74$ & $13.1 \pm 2.1$ & $12.2 \pm 3.6$ \\
\hline$\frac{v^{47}}{T_{i}^{45}} \frac{(p n)}{(a)}$ & $17.0 \pm 4.03$ & $48.5 \pm 7.7$ & $46.9 \pm 9.4$ \\
\hline$\frac{S c^{44}}{S c^{43}} \frac{(a p)}{(a p n)}$ & $11.8 \pm 2.80$ & $35.0 \pm 9.2$ & $32.4 \pm 10.6$ \\
\hline$\frac{\mathrm{V}^{47}}{\mathrm{Sc}^{43}} \frac{(p n)}{(a p n)}$ & $10.0 \pm 2.40$ & $129.3 \pm 33.6$ & $124.6 \pm 37.2$ \\
\hline
\end{tabular}

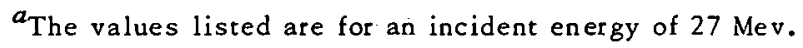

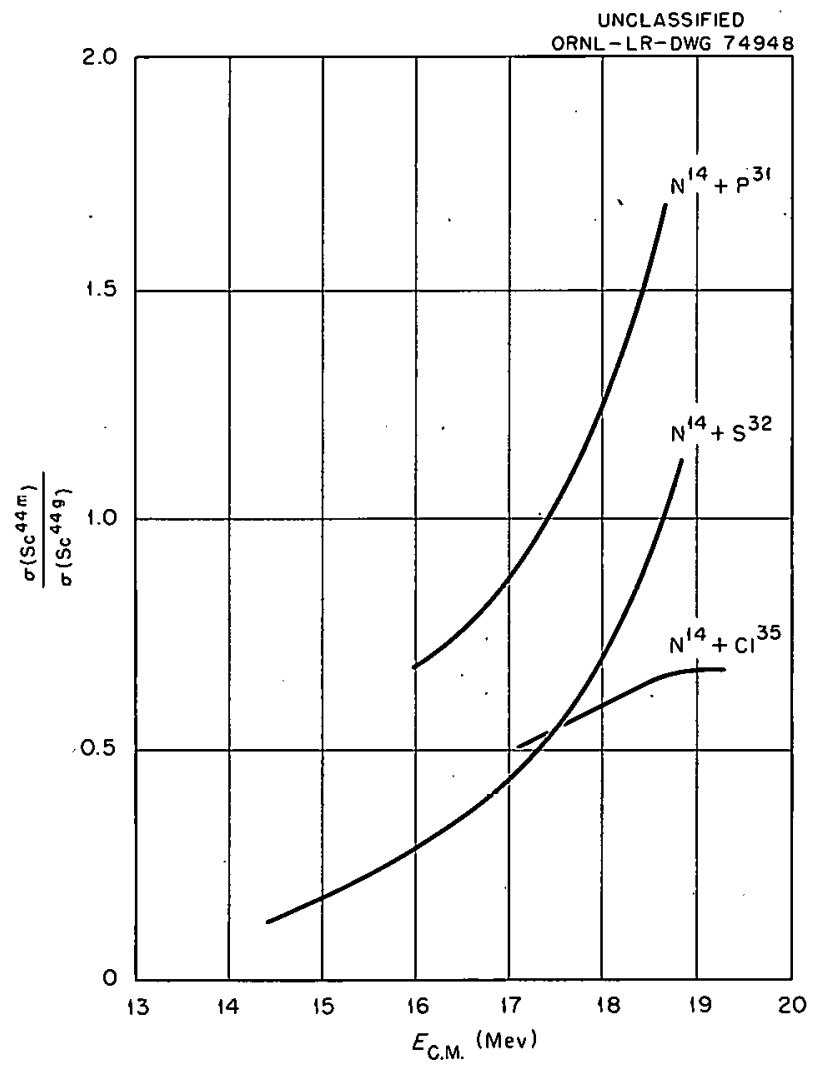

Fig. 1.2. Isomeric Yield Rafios of $\mathrm{Se}^{44}$ Resulting from the $\mathrm{N}^{14}$ Bombardment of $\mathrm{P}^{31}, \mathrm{~s}^{32}$, and $\mathrm{Cl}^{35}$. carried off by the alpha particle, should result in a higher yield of $\mathrm{Sc}^{44 \mathrm{~g}}$ from the chlorine reaction than from the $\mathrm{S}^{32}$ and $\mathrm{P}^{31}$ reactions. Secorid, perhaps some reaction mechanism other than compound-nucleus formation is beginning to set in at $\sim 18.6 \mathrm{Mev}$. Such a mechanism could account for the difference found between calculations and experimental results discussed in the previous section.

The ratio is higher for the $\mathrm{P}^{31}$ reaction than for the $S^{32}$ reaction at all energies investigated. Since both reactions bring about the same average angular momentum into the system (seven or eight units), the number of particles emitted from the compound nucleus in the two reactions should account for this difference. The proton emitted in the phosphorus reaction would carry off less angular momentum than the two protons emitted in the sulfur reaction. One should then predict a higher yield of the high-spin isomer in the system left with more angular momentum - in this case the system produced in the $\mathrm{P}^{31}$ reaction - which indeed is observed.

\section{Transfer Reactions}

The study of transfer reactions in the field of heavy-ion interactions is prompted by the desirability of obtaining information concerning the 
nuclear surface. Specifically, these reactions should ultimately result in the reduced widths of the transferring nucleon in the initial and final states.

The following five transfer reactions were investigated: $\quad \mathrm{K}^{39}\left(\mathrm{~N}^{14}, \mathrm{O}^{15}\right) \mathrm{Ar}^{38}, \mathrm{Cl}^{35}\left(\mathrm{~N}^{14}, \mathrm{O}^{15}\right) \mathrm{S}^{34}$, $\mathrm{Cl}^{35}\left(\mathrm{~N}^{14}{ }^{15} \mathrm{~N}^{15}\right) \mathrm{Cl}^{34 m}, \quad \mathrm{~S}^{32}\left(\mathrm{~N}^{14}, \mathrm{O}^{15}\right) \mathrm{P}^{31}$, and $\mathrm{Al}^{27}\left(\mathrm{~N}^{14}, \mathrm{O}^{15}\right) \mathrm{Mg}^{26}$. The excitation functions are displayed in Fig. 1.3.

In addition to predicting the shape of the angular distributions for transfer reactions, Breit. and collaborators ${ }^{7-9}$ derived an expression for the variation of the total cross section with energy. This expression is

$\sigma=\frac{(n \mathcal{N})^{2}}{2}\left(\frac{1}{a \overline{\bar{a}} \lambda \lambda^{\prime}}\right)$

$$
\times\left[\frac{\overline{b_{1} b_{2}}}{\left(1+a b_{1}\right)\left(1+\bar{a} b_{2}\right)}\right]^{2} \exp (x),(1)
$$

where

$$
\begin{array}{r}
x=\frac{\sqrt{2 M}}{b}\left[\left(b_{1}+\dot{b}_{2}\right) E_{s}^{1 / 2}\left(1-\frac{E_{B}}{E}\right)\right. \\
\left.+\left(\bar{b}_{1}+\bar{b}_{2}\right) \bar{E}_{s}^{1 / 2}\left(1-\frac{\bar{E}_{B}}{\bar{E}}\right)\right],
\end{array}
$$

$b_{1}, b_{2}=$ radii of initial and final nuclei 1 and 2 respectively,

$K=\hbar / M v=$ reduced wavelength of the transferred neutron,

$v=$ relative velocity,

$E_{s}=$ binding energy of the neutron,

$\alpha=\left(2 M E_{s} / \not\right)^{1 / 2}$,

$M=$ neutron mass,

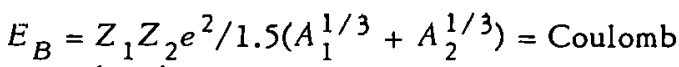
barrier,

$E=$ center-of-mass energy,

${ }^{7} \mathrm{G}$. Breit, Handbucb der Physik, ed. by S. Flugge, vol. XLI, pt 1, pp 367-407, Springer-Verlag, Berlin, 1959.

${ }^{8}$ G. Breit, pp $1-15$ in Proceedings of the Second Conference on Reactions Between Complex Nuclei, ed. by Alexander Zucker, E. C. Halbert, and F. T. Howard, Wiley, New York, 1960.

${ }^{9}$ G. Breit and M. E. Ebel, Pbys. Rev. 103, 679 (1956); 104,1030 (1956).

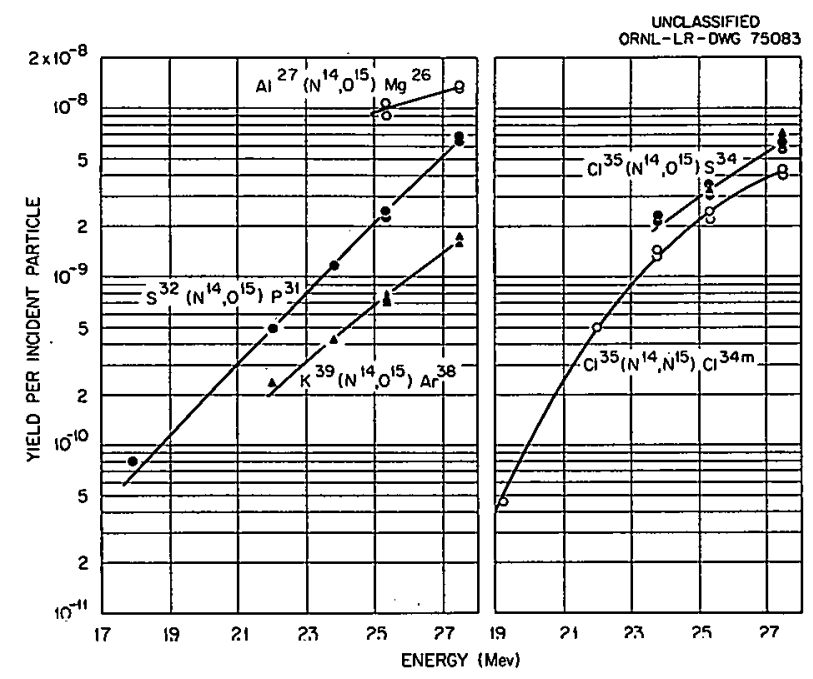

Fig. 1.3. Thick-Target Yields Determined for the Investigated Transfer Reactions. For the reaction $\mathrm{Cl}^{35}\left(\mathrm{~N}^{14}, \mathrm{O}^{15}\right) \mathrm{S}^{34}$, the closed dots show the dato obtained with targets containing natural-abundance chlorine; the closed triangles represent the data from targets enriched in $\mathrm{Cl}^{35}$.

and the unbarred and barred quantities refer to the initial and final systems respectively.

In addition to the various kinematical factors, the cross-section expression contains the product of the reduced widths of the neutron in the two participating nuclei $\lambda \lambda^{\prime}$, where $\lambda$ refers to the nucleus donating the neutron, and $\lambda^{\prime}$ refers to the nucleus which has accepted the transferred neutron. Specifically, for the case $\mathrm{B}^{10}\left(\mathrm{~N}^{14}, \mathrm{~N}^{13}\right) \mathrm{B}^{11}$, $\lambda$ refers to the neutron-reduced width in $\mathrm{N}^{14}$, while $\lambda^{\prime}$ refers to the $\mathrm{B}^{11}$. The probability of finding the neutron in a shell of unit thickness around one of these nuclei is proportional to the reciprocal of the appropriate $\lambda$.

To facilitate comparison between theory and experiment, the excitation functions can be plotted as $\log (\sigma / X)$ vs $x$. In the region where the tunneling theory is applicable $\left(E<E_{B}\right)$, it predicts that $x$ should change by a factor of $\ln 10$ for an order of magnitude change in $\sigma / \mathbb{K}^{2}$.

The available experimental data on singlenucleon transfers ${ }^{10}$ have been grouped into three

\footnotetext{
${ }^{10}$ For a complete list of references see K. S. Toth and Eugene Newman, to be published in the Pbysical Re. view.
} 
sections: (1) $\mathrm{N}^{14}$-induced reactions producing $\mathrm{N}^{13}$ as one of the reaction products, (2) $\mathrm{F}^{19}$. induced transfers resulting in $F^{18}$, and (3) the five reactions investigated during this past year, together with two single-proton transfers studied earlier. These data are plotted in Fig. 1.4. Although the excitation functions, as plotted, obviously do not all have the same slopes, there is a tendency for the heavier-target data to fall on straight lines that group together and have rather similar slopes, close to the value predicted by the tunneling theory. Two targets, $\mathrm{B}^{10}$ and $\mathrm{N}^{14}$, behave anomalously in all three sets of data, the anomaly being manifested by a slope that is much more gentle than the one predicted by the tunneling theory and/or by a break in the curves. Another reaction, $\mathrm{Cl}^{35}\left(\mathrm{~N}^{14}, \mathrm{~N}^{15}\right) \mathrm{Cl}^{34 m}$, involving the detection of the isomer of $\mathrm{Cl}^{34}$, also displays a break in the excitation function.

There are four pairs of reactions in which the same target nucleus has been bombarded with both $\mathrm{N}^{14}$ and $\mathrm{F}^{19}$ beams. The product $\lambda \lambda^{\prime}$ can be extracted from the value of $\sigma / \AA^{2}$ at the point where $x=0$. For the above-mentioned pairs, however, because of the identical targets, four independent values of the ratio $\lambda\left(\mathrm{N}^{14}\right) / \lambda\left(\mathrm{F}^{19}\right)$ can be obtained. Naturally, these four ratios must be identical and serve as a test as to how well the $\lambda$ 's may be extracted from the curves. In Table 1.2 the reactions, the $\lambda \lambda^{\prime}$ products, and the $\lambda\left(\mathrm{N}^{14}\right) / \lambda\left(\mathrm{F}^{19}\right)$ ratios are listed. Two entries for the $\mathrm{Al}^{27}\left(\mathrm{~N}^{14}, \mathrm{~N}^{13}\right) \mathrm{Al}^{28}$ reaction are shown. The values shown are straightline extrapolations of the data of refs 11 and 12 respectively. The measurements of ref 12 exist only below $x=0$, whereas those of ref 11 were made at energies above $x=0$. Of the two $\lambda \lambda^{\prime}$ products the one deduced from ref 11 yields a ratio, $\lambda\left(\mathrm{N}^{14}\right) / \lambda\left(\mathrm{F}^{19}\right)$, which is closer to those derived from the remaining three pairs of reactions. It must be remarked, however, that both sets of data, refs 11 and 12, involve large experimental errors.

\footnotetext{
${ }^{11}$ V. V. Volkov, A. S. Pasiuk, and G. N. Flerov, Soviet Pbys. JETP' (English transl.) 6, 459 (1958).

12 W. H. Webb, H. L. Reynolds, and Alexander Zucker, Phys. Rev. 102, 749 (1956).
}
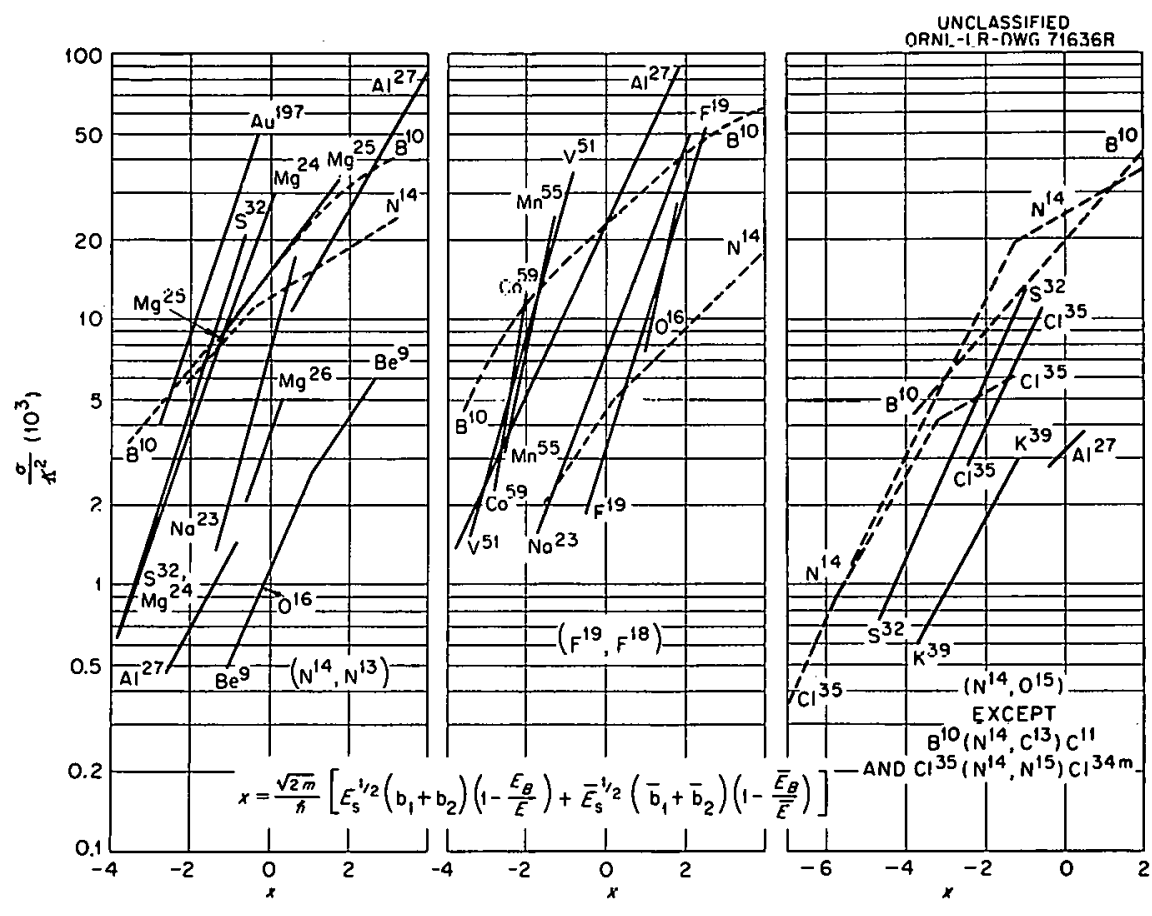

Fig. 1.4. Single-Nucleon Cross-Section Data Platted as $\sigma / A^{2}$ vs $x$. $A=\hbar / M \nu=$ the reduced wavelength of the transferred nucleon; $x=$ the quantity shown in the figure. The figure is divided into three portions as follows: at the left $\left(N^{14}, N^{13}\right)$ transfers; in the center $\left(F^{19}, F^{18}\right)$ transfers; and at the right the present data together with two proton transfers studied earlier. The curves are labeled according to the bombarded target. 
Table 1.2. Reduced Widths

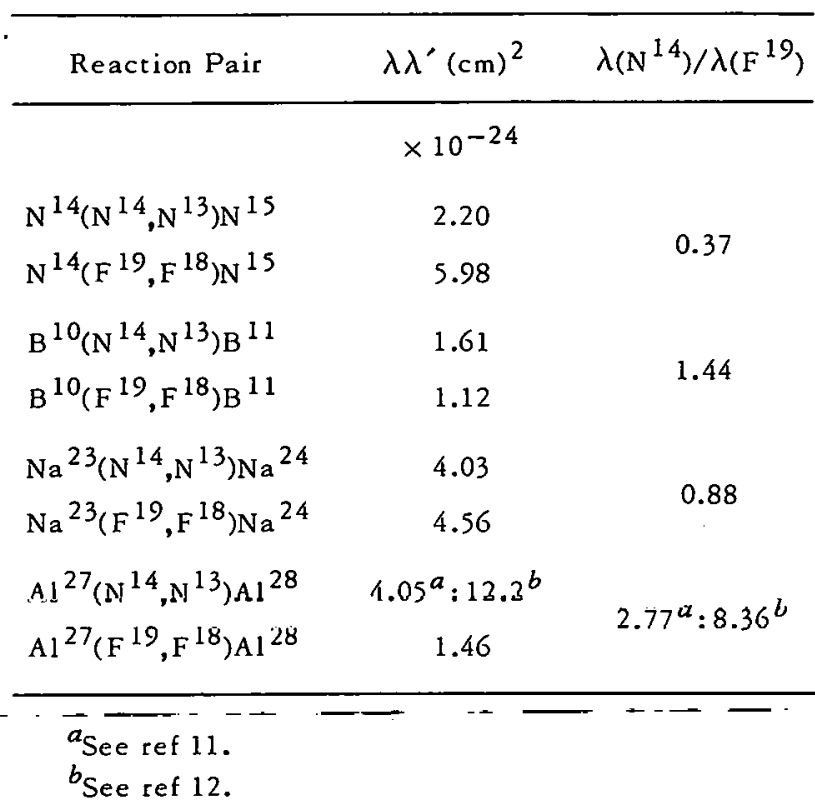

In ref 12 the measured cross section is extremely low, whereas in ref 11 the uncertainty in the $\mathrm{N}^{14}$ beam energy was approximately $10 \mathrm{Mev}$. Although the agreement among the four sets of ratios is not spectacular, it is gratifyingly close, especially since only kinematical factors and no detailed nuclear structure information were included in the tunneling model.

In an earlier attempt ${ }^{13}$ to systematize transfer excitation functions, the measured cross sections were plotted vs $E^{*}$, where $E^{*}=E_{\text {c.m. }}-E_{\text {barrier }}+$ $Q$. The quantity $E^{*}$ is the kinetic energy available to the system at the time of contact after the transfer has occurred. Excitation functions plotted in this manner, with a few exceptions, tend to group together. It was hoped that with a few exceptions a "universal curve" existed for transfer reactions and that it could be used to predict unknown cross sections by the calculation of the quantity $E^{*}$. Fisher et al., ${ }^{14}$ at the suggestion of Breit, replotted transfer cross sections vs the expression $E_{\text {c.m. }}-E_{\text {barrier }}+Q / 2$. Their "universal curve" brought some excitation functions closer together but displaced others.

The available single-nucleon transfer cross sections at low incident energies were plotted vs

\footnotetext{
${ }^{13}$ M. L. Halbert et al., Phys. Rev. 106, 251 (1957).

${ }^{14}$ D. E. Fisher, Alexander Zucker, and A. Gropp, Pbys. Rev. 113, 542 (1959).
}

$E^{*}$, as shown in Fig. 1.5. Dotted curves represent the five excitation functions obtained in this work, dashed curves show the data of Perkin et al., 15 and the solid curves represent the earlier data obtained with $\mathrm{N}^{14}$ beams. Not all excitation functions are shown in the diagram, because some curves overlap and if displayed would tend to be confusing. A sufficient number of curves are shown, however, so that it is readily seen that in fact no "universal curve" exists. Reactions whose $E^{*}$ values are about -7 or $-8 \mathrm{Mev}$ would have been expected, from the earlier "universal curve," to have extremely small cross sections; however, they are shown to possess cross sections of a few millibarns. Also, instead of being closely grouped, the curves arc spread over a wide range of $E^{*}$. A trend can be ovserved from Fig. 1.5; that

${ }^{15} \mathrm{~J}$. L. Perkin, R. F. Coleman, and D. N. Herbert, Proc. Phys. Soc. 79, 1033 (1962).

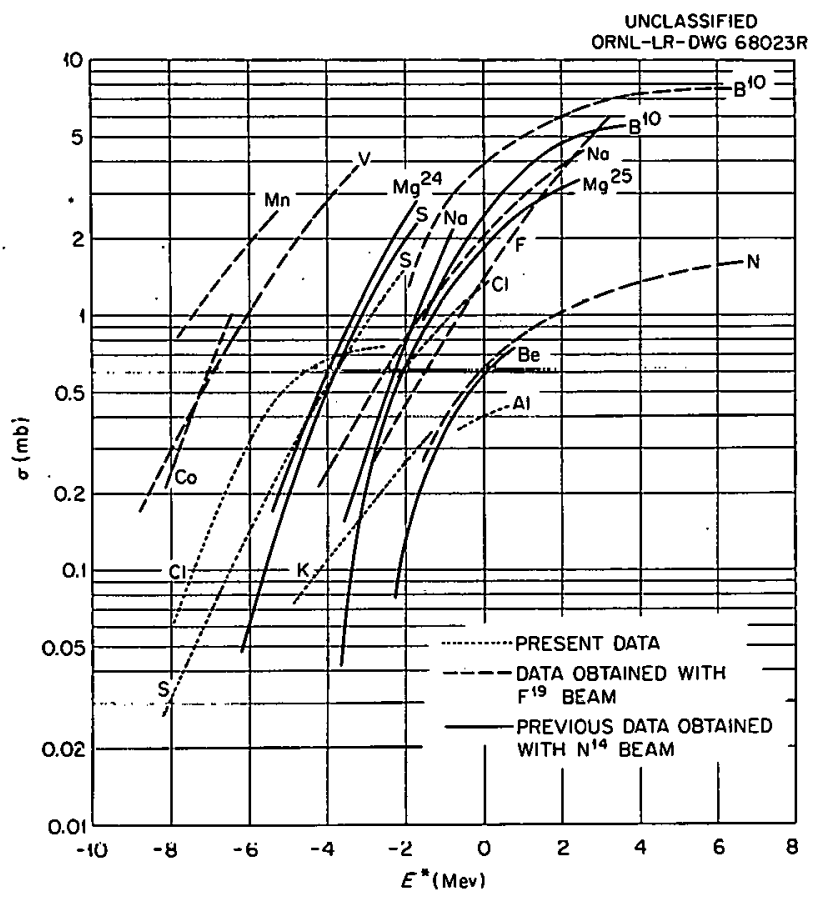

Fig. 1.5. Single-Nucleon Transfer Cross Sections Plotted as a Function of $E^{*}$, where $E^{\star}=E_{\text {c.m. }}$. $E_{\text {barrier }}+Q$. Dotted curves represent the five investigated reactions; dashed eurves show the data of Perkin et al.; and the full curves represent earlier data obtained with $\mathrm{N}^{14}$ beams. The curves are labeled according to the bomborded target. 
is, the higher the atomic number of the target, the higher the cross section for a given value of $E^{*}$. Plotting the cross sections in the manner of Fisher et al. ${ }^{14}$ does not bring about any additional clustering around a "universal curve."

\section{TRANSFER.REACTION ANGULAR DISTRIBUTIONS}

$$
A I^{27}\left(O^{16}, N^{15}\right) S_{i}^{28}
$$

The differential cross section of the proton transfer reaction $\mathrm{Al}^{27}\left(\mathrm{O}^{16}, \mathrm{~N}^{15}\right) \mathrm{Si}^{28}$ was measured by using an $\mathrm{O}^{16}$ beam of $30 \mathrm{Mev}$ from the ORNL Tandem Van de Graaff. For an assumed radius $r_{0}$ of 1.5 fermis, the incident energy is $0.78 \mathrm{Mev}$ below the Coulomb barrier. Two groups of $\mathrm{N}^{15}$ particles were observed; these were shown to result from transfers in which (1) both residual nuclei were left in their ground states $(Q=-0.54$ Mev), and (2) $\mathrm{N}^{15}$ was left in its ground state and $\mathrm{Si}^{28}$ in its first excited $(1.78 \mathrm{Mev})(Q=-2.32 \mathrm{Mev})$ state.

The target uised was an evaporated, selfsupporting aluminum film having a thickness of $125 \mu \mathrm{g} / \mathrm{cm}^{2}$. The detection system consisted of a scattered particle-recoil particle coincidence apparatus supplemented by a proportional counter to impose a further restriction on the nature of the scattered particle. ${ }^{16}$ The proportional counter portion of the scattered-particle identification system described in a previous progress report ${ }^{17}$ was replaced by a counter of different design. In the new detector the collector wire is on the axis of the cylinder, and the particles traverse midway between the wire and the wall, parallel to the axis. This arrangement improved the resolution by ap: proximately $5 \%$, thus providing better separation between particles of different nuclear charge.

Figure 1.6 shows the observed angular distributions. It is seen that the ground-ground (G-G) transfer cross section is larger than the G-lst excited-state cross section in the forward direction but smaller on the high-angle side of the peak. Both distributions peak at approximately the same angle and with the same differential cross section.

\footnotetext{
${ }^{16}$ Eugene Newman, Phys. Reu. 125, 600 (1962).

${ }^{17}$ Electronuclear Research Div. Ann. Progr. Rept. Jan. 16, 1961, ORNL-3083, p 11.
}

The integrated cross sections are 1.3 and $1.6 \mathrm{mb}$ for the G-G and G-1st transfers respectively.

The angular distributions were compared with the predictions of the tunneling theory of Breit, bearing in mind that the theory, as formulated, applies to the transfer of neutrons and not protons. To facilitate comparison with the theory and to permit extraction of the interaction radius, the distributions were replotted ${ }^{17}$ as $d \sigma / d R_{\min }$ vs $R_{\min }$, shown in Fig. 1.7. The radius parameters, $r_{0}$, of $1.81 \pm 0.04$ and $1.73 \pm 0.04$ fermis were deduced for the G-G and G-1st distributions respectively. The solid lines are the predictions of the tunneling theory normalized to the data. It is apparent that the agrecment is far better for the G-lst distribution than for the G.G.

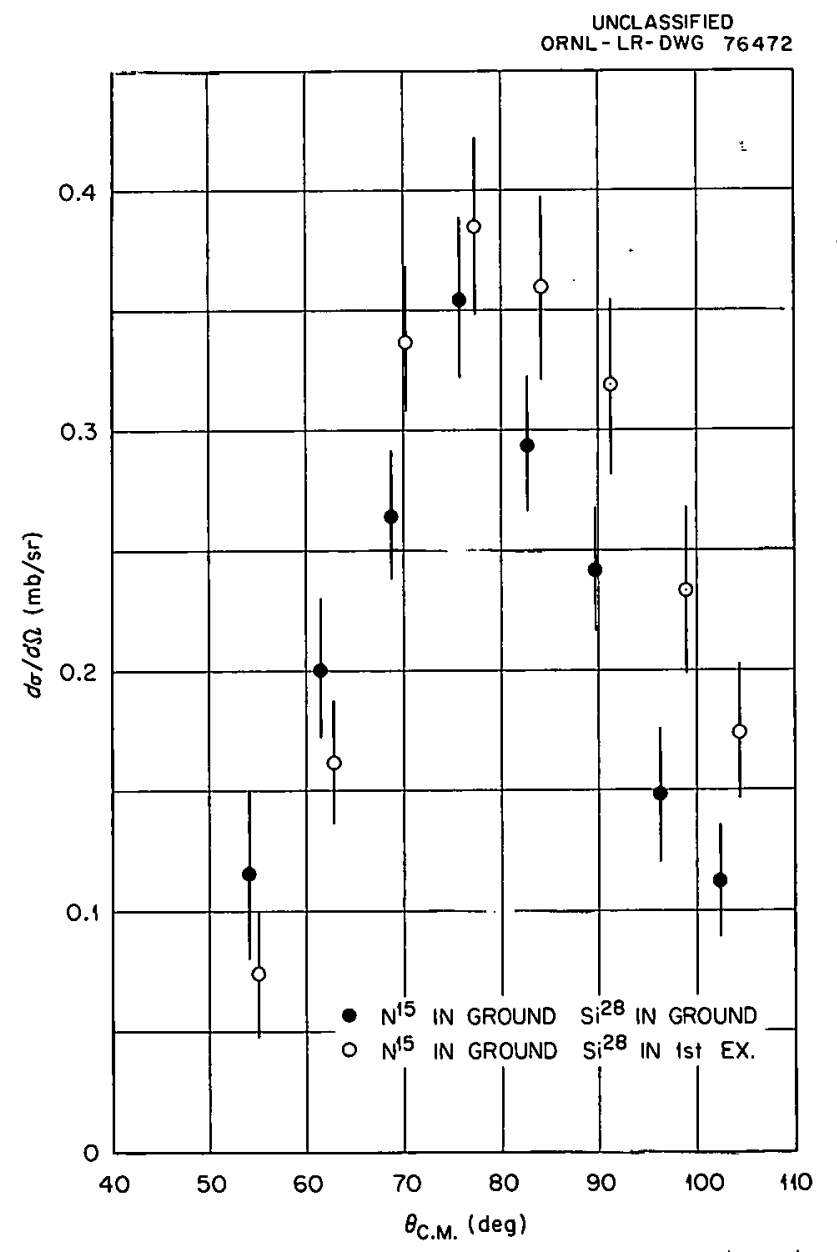

Fig. 1.6. Differential Cross Section for the Reaction $A)^{27}\left(O^{16}, N^{15}\right) S^{28}$. 


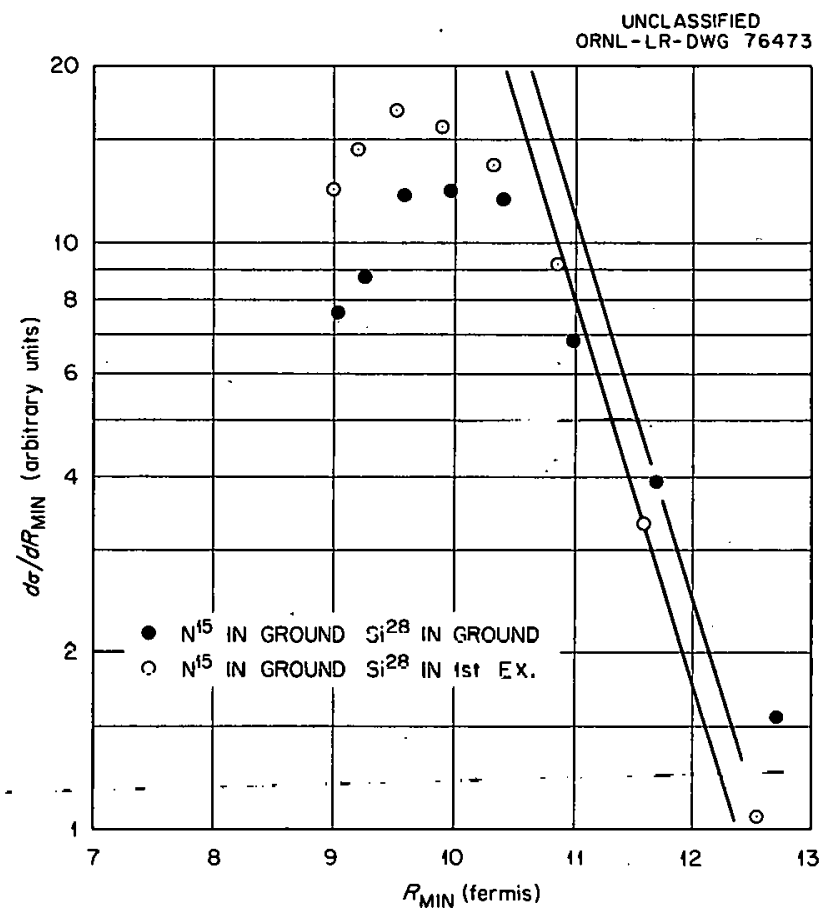

Fig. 1.7. Transformed Differential Cross Section for the Reaction $\mathrm{Al}^{27}\left(\mathrm{O}^{16}, \mathrm{~N}^{15}\right) \mathrm{Si}^{28}$. Solid lines are the predictions of the tunneling theory normalized to the experimental data.

\section{Complex-Transfer Distributions}

In a recent publication, $\mathrm{Kammuri}^{18}$ was able to reproduce the angular distribution for complex transfers by a simple semiclassical calculation. These reactions, which involve the transfer of many particles from the projectile to the target, and vice versa, have been observed by Kaufmann and Wolfgang. ${ }^{19}$ They are characterized by strong forward peaking and cross sections at incident energy of the order of $10 \mathrm{Mev} /$ nucleon, approaching those of single-nucleon transfers.

Briefly, the approach taken by Kammuri is as follows. It is assumed that the se complex transfers occur in the nuclear surface region and that the projectile passes through this region in a Coulomb orbit. With the JWKB approximation, the

\footnotetext{
${ }^{18} \mathrm{~T}$. Kammuri, Proceedings of the Intemational Symposium on Direct Interactions and Nuclear Reaction Mechanisms, Padua, Italy, 1962, in press.

${ }^{19} \mathrm{R}$. Kaufmann and R. Wolfgang, Pbys. Rev. 121, 206 (1961).
}

classical scattering function $\Theta(l)$ is calculated from a Saxon potential. By obtaining the phase shifts from $\Theta(l)=2 d \eta / d l$ over a narrow region of $l$, the scattering amplitude is calculated in the usual way; that is,

$$
\begin{gathered}
f(\theta)=\frac{1}{2} i k \sum_{l-\Delta}^{l+\Delta}(2 l+1)\left(e^{2 i \eta_{l}}-1\right) P_{l}(\cos \theta), \\
\sigma(\theta)=P|f(\theta)|^{2} .
\end{gathered}
$$

Good agreement with the data is obtained for $l=76$ and $\Delta=2$ or $\Delta=4$, as may be seen in Fig. 1.8 .

We have made the same calculation, except that only Coulomb phase shifts were used. The fit to the data for $l=76$ and $\Delta=2$ is as acceptable as for the calculations above, as seen by the dashed.. lines; liowever, the fit is destroyed for $\Delta=4$. The interaction radius corresponding to $l=76$ is 9.8 fermis, or $r_{0}=1.31$ fermis. This means that the

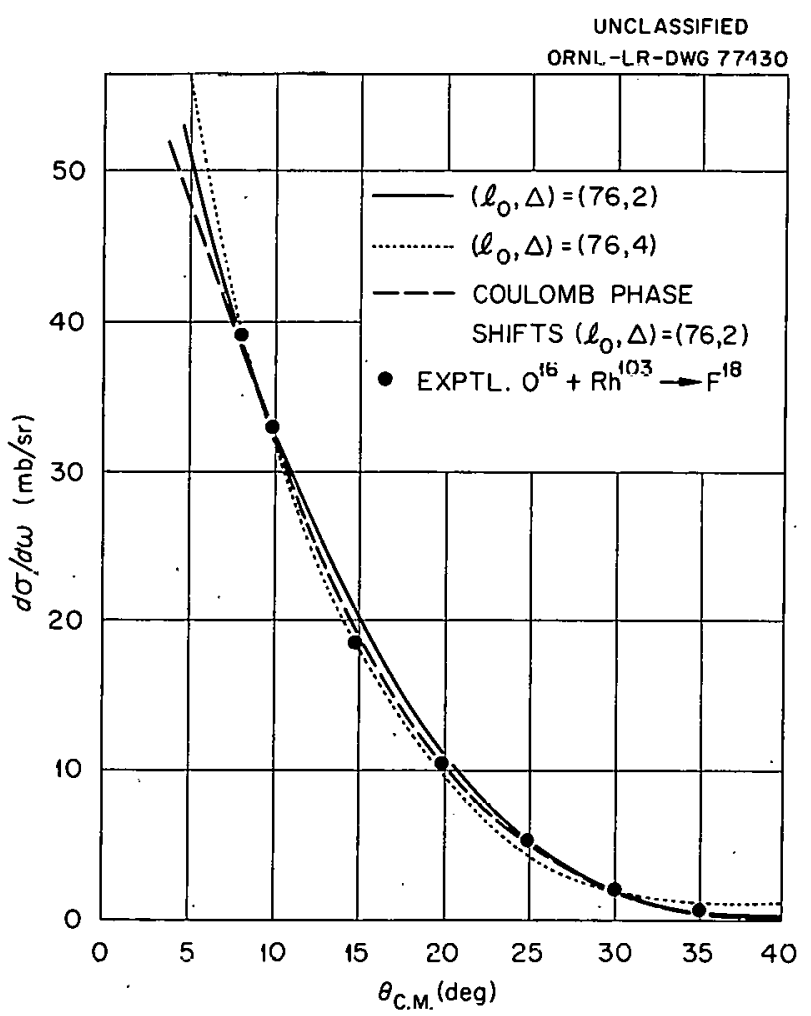

Fig. 1.8. Measured Angular Distribution of $F^{18}$ from the Reaction of $\mathrm{O}^{16}+\mathrm{Rh}^{103}$, Along with Various Theoretical Predictions. 
colliding nuclei pass through rather dense regions of nuclear matter and yet retain their elastic orbits, or, in the second calculation, feel principally a Coulomb potential.

\section{ANGULAR-MOMENTUM EFFECTS}

On fairly general grounds it. is expected that the density of nuclear levels as a function of spin $j$ does not continue to increase as $(2 j+1)$ for large spin, but instead is subject to a strong cutoff. A limitation on the number of levels with high spin in the final nucleus may manifest itself in many ways. Among the must striking are strong forwardbackward peaking of the emitted particles and failure of the independence hypothesis for formation and decay of the compound nucleus.

The $28-\mathrm{Mev} \mathrm{N}^{14}$ ions accelerated in the ORNL 63-Inch Cyclotron are well suited for studies of the nuclear spin distribution, since they bring in orbital angular momenta typically as high as $l=12$. The two efferts mentioned above were observed in reactions producing alpha particles from bombardment of $\mathrm{C}^{12}, \mathrm{O}^{16}, \mathrm{Na}^{23}$, and $\mathrm{Al}^{27}$ by $\mathrm{N}^{14}$ ions. The experimental work is described and some results are presented in ORNL-3257. Additional angular distributions are shown in Figs. 1.9-1.11. Each shows the expected symmetric forwardbackward peaking.
The experimental data provide information about the dependence of the residual-nucleus level density on excitation energy and spin. Theoretical expressions for the compound-nucleus cross section to a given final channel have been worked out in a semiclassical approximation by Ericson and Strutinski 20,21 and quantum mechanically by Douglas and MacDonald. ${ }^{22}$ In each of these treatments the level density at excitation energy $E^{*}$ for spin $j$ is assumed to have the form

$$
\rho\left(E^{*}, j\right)=\text { const } \rho\left(E^{*}\right)(2 j+1) e^{-j(j+1) / 2 \sigma^{2}},
$$

where $\rho\left(E^{*}\right)$ is the total density of levels at this energy. The spin cutoff parameter $\sigma$ is a slowly varying function of $E^{*}$. It may be used to define a ment of inertia $d$ according to

$$
\sigma^{2}=d T / b^{2}
$$

where the nuclear temperature $T$ is given by

$$
1 / T=d \ln \rho\left(E^{*}\right) / d E^{*} .
$$

${ }^{20}$ T. Ericson and V. Strutinski, Nucl. Phys. 8, 284 (1958); 9, 697 (1959).

21 T. Ericson, Adyan. Phys. 9, 425 (1960).

22 A. C. Douglas and N. MacDonald, Nucl. Phys. 13, 382 (1959).

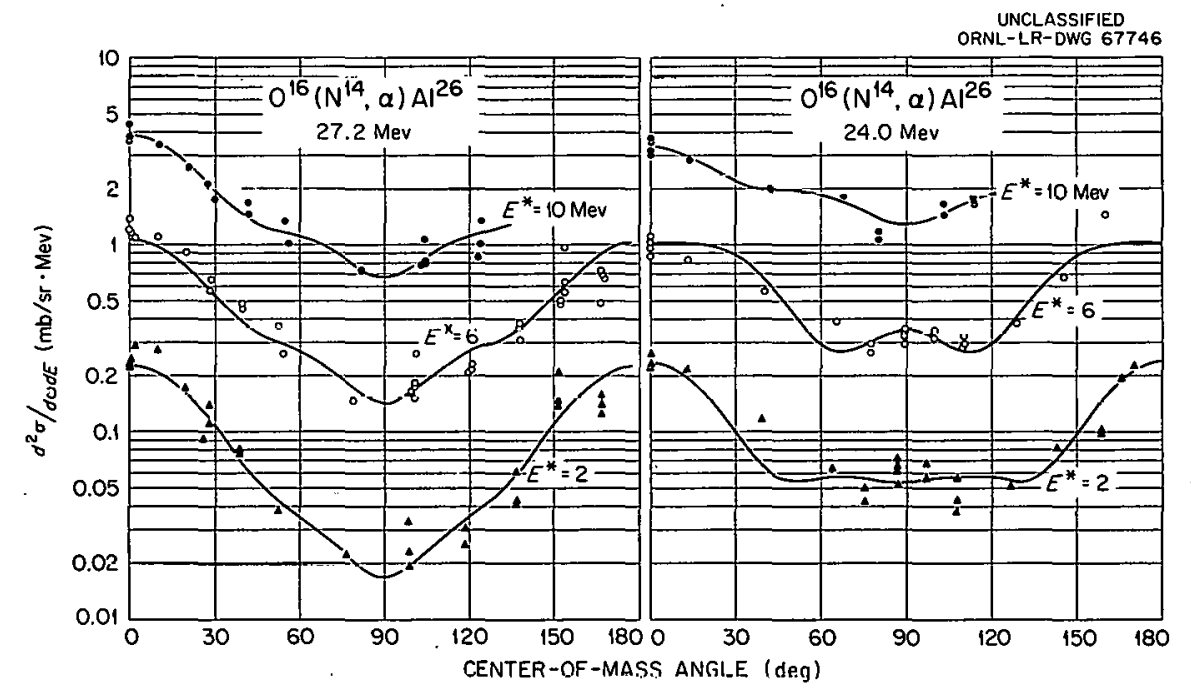

Fig. 1.9. Differential Cross Sections for $\mathrm{O}^{16}\left(\mathrm{~N}^{11}, \alpha\right) \mathrm{Al}^{26}$ for Various Choices of Al ${ }^{26}$ Excitation Energy E $E^{k}$ at Mean Bombarding Energies of 27.2 and 24.0 Mev. 


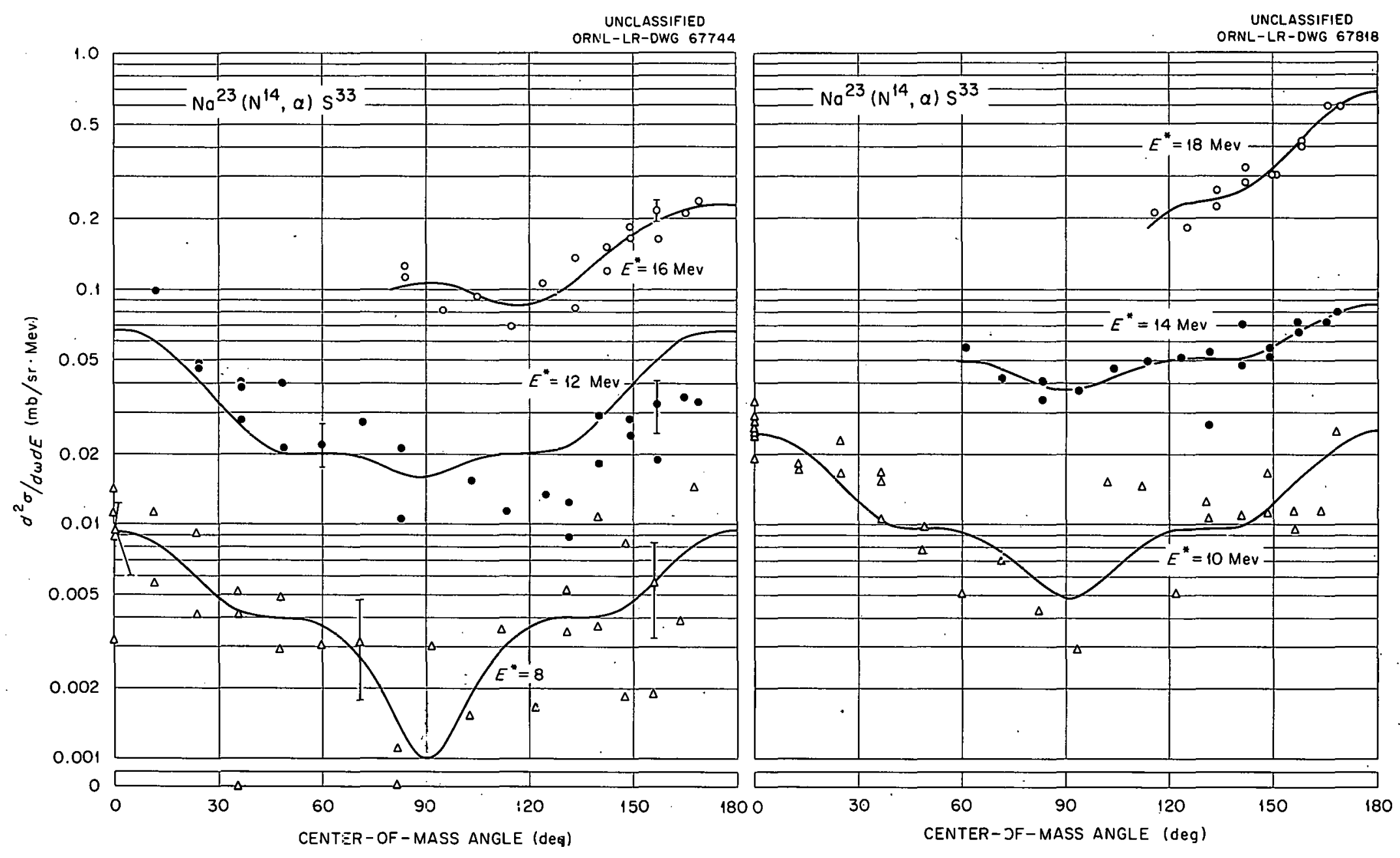

Fig. i.10. Differential Cross Sections for $\mathrm{Na}^{23}\left(\mathrm{~N}^{14}, a\right) \mathrm{s}^{33}$ at 26.7. Mev Mean Bombarding Energy. Points are missing from some curves at forward angles because alpha particles from carbon imfurity in the target obscured the alphas from sodicm. 


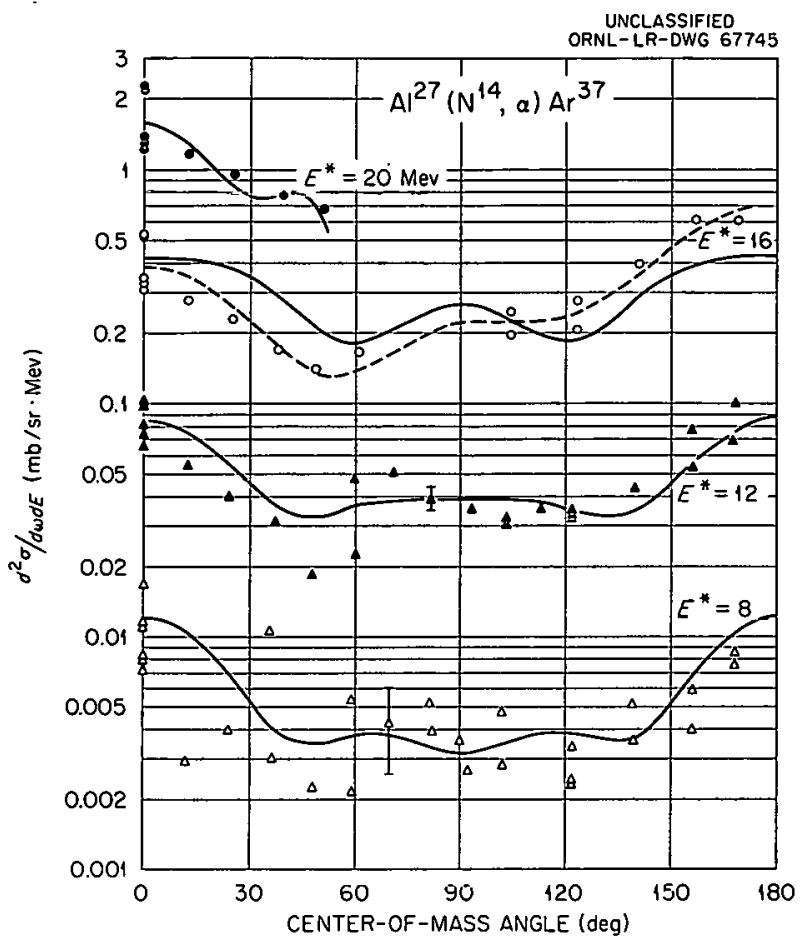

Fig. 1.11. Differential Cross Sections for the Reaction $\mathrm{Al}^{27}\left(\mathrm{~N}^{14}, \alpha\right) \mathrm{Ar}^{37}$ at $26.6 \mathrm{Mev}$ Mean Bombarding Energy.

In both theories the final result is of the form

$$
\frac{d^{2} \sigma}{d E^{*} d \omega}=\sum_{n(\text { e ven })} C_{n} P_{n}(\cos \theta) .
$$

For comparison with the theory, all the experimental angular distributions were fit with expressions of the above form by the method of least squares. The smooth curves in Figs. 1.9-1.11 are fits for $n$ up to 6 .

The semiclassical approximation is valid for reactions with $\mathrm{N}^{14}$. Since it appeared that the approximate theory would be simpler to evaluate than the quantum version, attention was concentrated on calculating the $C_{n}$ by using the semiclassical theory [Eq. (5.26) of ref 21]. If this were done for a number of choices of $\sigma$ at a given $E^{*}$, comparison with the least-squares fits to the data would then determine the best value of $\sigma$ for that $E^{*}$.

When large angular momenta are involved, even the approximate theory becomes very timeconsuming to evaluate for cach value of $\sigma$; hand calculations cannot be considered for a thorough exploration of $\sigma$ values at each value of $E^{*}$ for each reaction. Consequently, a FORTRAN program was written for the IBM 7090 to perform these calculations. The program has been used thus far to analyze some of the data obtained with the $\mathrm{O}^{16}$ target. The data for the region $E^{*}=6$ to $12 \mathrm{Mev}$ in the residual nucleus $A l^{26}$ is consistent with $\sigma=$ $2.5 \pm 0.5$, which corresponds to $d \approx 0.7 d_{\text {rigid }}$.

Results have also been obtained for the variation of the total level density with excitation energy. Each data point in an energy spectrum was divided by the available phase space and the inverse cross section. Figures 1.12 and 1.13 give examples of these fits for two choices of level density,

$$
\begin{aligned}
& \rho_{1}\left(E^{*}\right)=C_{1} e^{2 \sqrt{a\left(E^{*}-B\right)}}, \\
& \rho_{2}\left(E^{*}\right)=\frac{C_{2} e^{2 \sqrt{a\left(E^{*}-B\right)}}}{\left(E^{*}-B\right)^{2}} .
\end{aligned}
$$

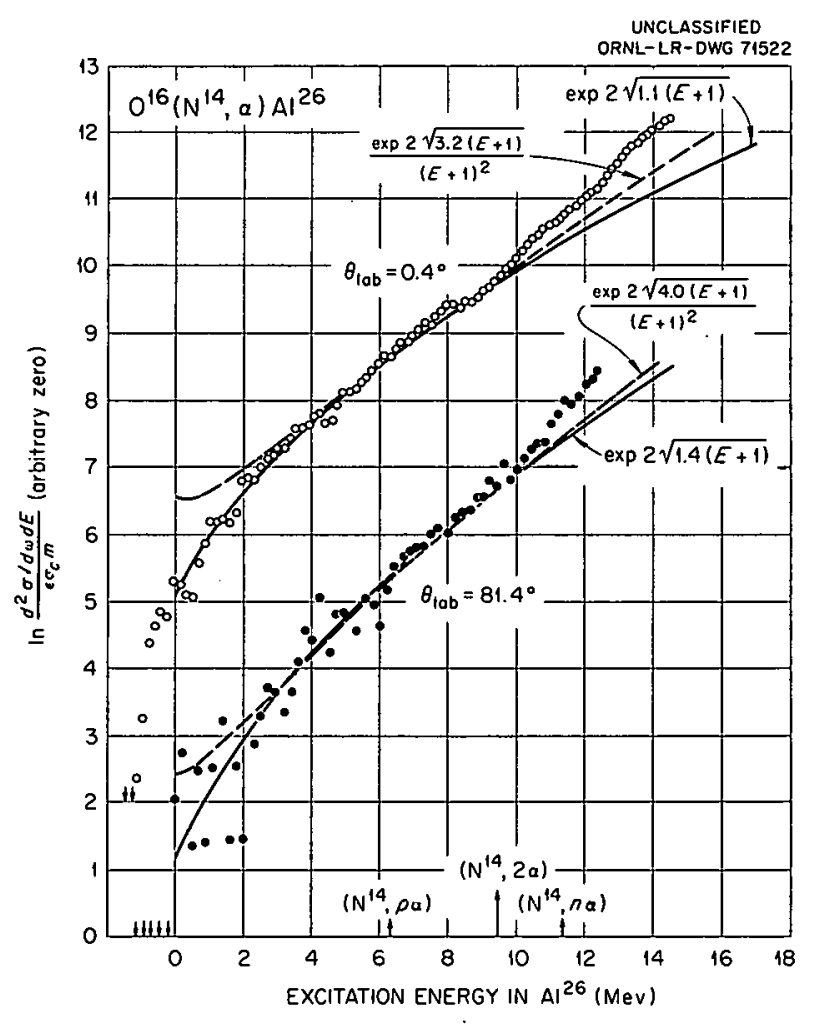

Fig. 1.12. Level-Density Determinations at Two Angles for $\mathrm{Al}^{26}$. Fits to $\rho_{1}\left(E^{\star}\right)$ and $\rho_{2}\left(E^{\star}\right)$ are indicated. The thresholds for reactions in which the observed alpho particle might not have been the first one emitted are shown on the energy scale. 


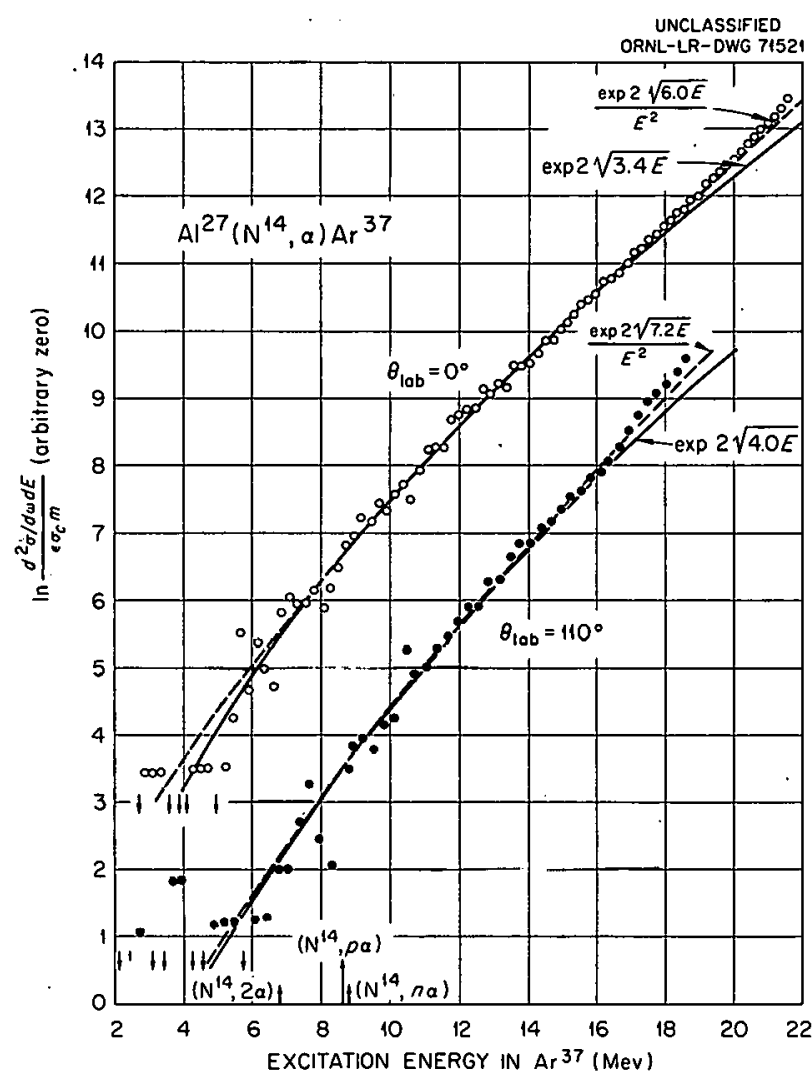

Fig. 1.13. Level-Density Determinations af Two Angles for $\mathrm{Ar}^{37}$.

For the $U^{16}$ target, $B=-\overline{1}$ gave the best fit; for the others, $B=0$,

Figures 1.14-1.16 show the apparent values of $a$ at each angle for three of the reactions. The word "apparent" was used to signify that the variation of $a$ with angle does not mean that the level density really changes with angle. Rather, the variation arises because the method of analysis is based on the independence hypothesis, which fails when angular momentum is limited.

The angular-distribution results for the spin cutoff parameter are insensitive to the choice of level-density parameters. For example, doubling the parameter $a$ in the level density $\rho_{1}\left(E^{*}\right)$ for the alpha-particle channel is equivalent to a reduction

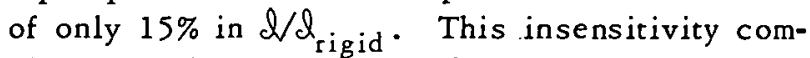
plements that found for the effect of angularmomentum limitation on the apparent level density, as shown in Figs. 1.14-1.16. That is, although the apparent value of the parameter $a$ is a function of angle, the variation is relatively small. It is extremely fortunate that the two effects can be separated so clearly; otherwise, it would have been a hopeless task to determine either $a$ or $\sigma$ from these experiments.

A few comments about the calculations of the theoretical angular distributions follow. For each choice of $\sigma$ the program for the semiclassical formula calculates all the even-order Legendre coefficients $C_{n}$ up to some value $n_{\max }\left(n_{\max } \leqq 18\right)$ for excitation energies spaced any integral number of Mev apart. Options are included which permit $d / d_{\text {ingid }}$, instead of $\sigma$, to be held constant or to be varied with $E^{*}$ in a simple way. The energy dedependence of $\rho\left(E^{*}\right)$ may also be varied from run to run, if dcsired; four options are available. The $\sigma$ and $\rho$ parameters for each of the possible exit channels (maximum number 5) are specified individually. Incident and exit-channel angular momenta up to $29 \hbar$ are allowable.

In addition to the $C_{n}$, the program also prints out certain intermediate results of physical interest. For example, the total width for compound-nucleus decay and the relative widths for each exit channel

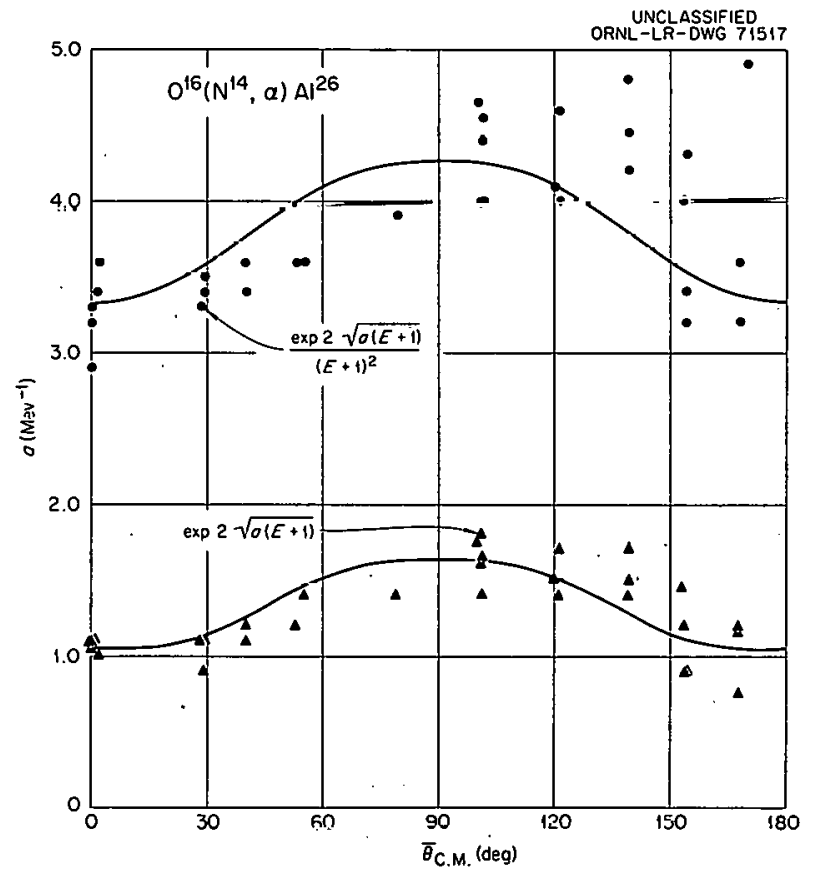

Fig. 1.14. Best-Fit Parameters as a Function of Angle for the Total Level Density $\rho_{1}\left(E^{\star}\right)$ and $\rho_{2}\left(E^{\star}\right)$ for Al $^{26}$. 


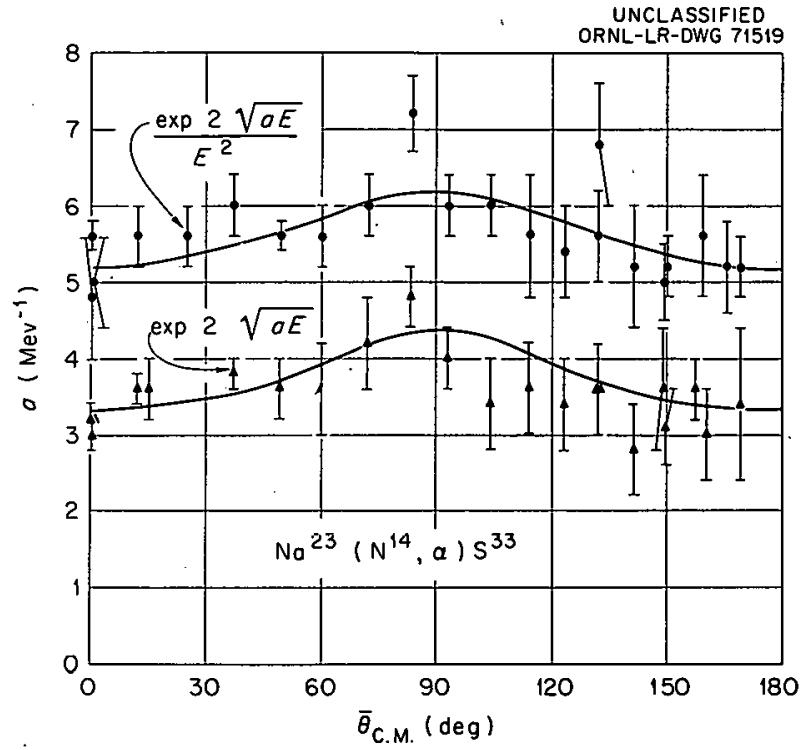

Fig. 1.15. Best.Fit Parameters as a Function of Angle for the Total Level Density $\rho_{1}\left(E^{\star}\right)$ and $\rho_{2}\left(E^{\star}\right)$ for $\mathrm{s}^{33}$.

are printed out as a function of the angular momentum $l$ of the compound nucleus.

The program calls for a set of transmission coefficients for all exitchannels at I-Mev intervals in the channel energy. In the present work these are contained in decks of cards which are punched automatically by the use of a separate program based on the optical-model code of R. H. Bassel and R. M. Drisko of this Division. Well parameters were selected from fits to elastic-scattering data obtained by other investigators.

It seems clear now that the approximate formula is actually more difficult for the computer to evaluate than the exact theory. The former includes spherical Bessel functions of pure imaginary argument which are the classical analogs of certain combinations of Clebsch-Gordan and Racah coefficients. Thesc functions incrcase without limit for large argument; great care is necessary in programming to avoid severe loss of significant digits and overflow; while at the same time minimizing the computing time. The subprogram finally developed selects downward recursion, upward recursion, or a power-series expansion for the computation, according to the values of the argument and of $n_{\text {max }}$. It is accurate to at least 1 part in $10^{6}$. Running time of the entire program on

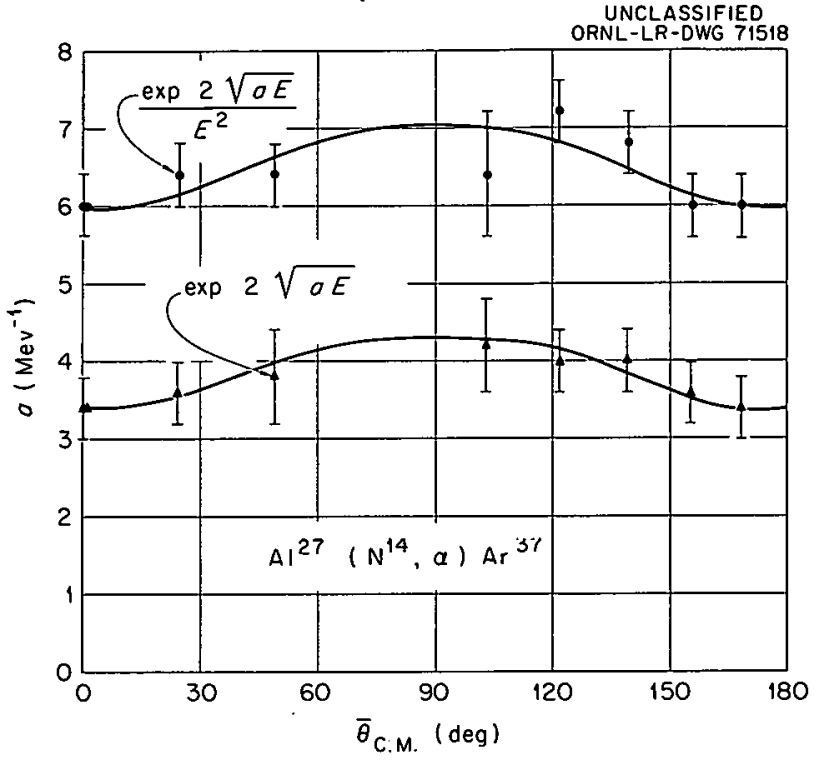

Fig. 1.16. Best-Fit Parameters as a Function of Angle for the Total Level Density $\rho_{1}\left(E^{\star}\right)$ and $\rho_{2}\left(E^{\star}\right)$ for $\mathrm{Ar}^{37}$.

the IBM 7090 for calculations at 11 values of excitation energy is $1.6 \mathrm{~min}$ for $n_{\mathrm{max}}=8$ and 2.4 $\min$ for $n_{\max }=14$.

The quantum-mechanical version is now being programmed, also in FORTRAN. All the $Z$ coefficients needed have already been calculated for angular momenta up to 29 by a code incorporating expanded versions of the Clebsch-Gordan and Racah subroutines of $A$. Culkowski (Mathematics Division). They are stored on magnetic tape and will be transferred to the computer memory in large blocks when required by the main program. The quantum computation should take far less time than the semiclassical formula.

\section{RESONANCES IN THE $\mathrm{C}^{12}\left(\mathrm{O}^{16}, a\right) \mathrm{Mg}^{24}$ REACTION}

Recently, attention has been called ${ }^{23}$ to resonances in the excitation functions for $(p, a), 24,25$

\footnotetext{
${ }^{23}$ U. Facchini, E. Saetra Menichella, and F. Tonolini, Phys. Letters 1, 209 (1962).

${ }^{24} \mathrm{H}$. Ogata et al., J. Phys. Soc. Japan 15, 1719 (1060).

${ }^{25}$ G.'E. Fischer et al., Phys. Rev. 110, 286 (1958).
} 
$(n, a),{ }^{26}(d, \alpha),{ }^{27}$ and $(d, p)^{28}$ reactions in the region $Z=12$ to 14 . The observed structure, approximately $150 \mathrm{kev}$ wide, is prominent even at $25 \mathrm{Mev}$ excitation in the compound system, where levels are expected to overlap and smoothly varying cross sections might have been anticipated. Ericson pointed out ${ }^{29}$ that such fluctuations are to be expected from the statistical theory of compound-nucleus decay and that under certain conditions they provide a measure of the compoundnucleus lifetime.

To determine whether the structure is restricted to nucleon- and deuteron-induced reactions, we have measured the excitation functions for $\mathrm{C}^{12}\left(\mathrm{O}^{16}, a\right) \mathrm{Mg}^{24}$ in two energy regions: from 23.9 to $26.0 \mathrm{Mev}$ and from 29.6 to $30.9 \mathrm{Mev}$ excitation in the compound nucleus $\mathrm{Si}^{28}$. Measurements were made at laboratory angles of 30 and $150^{\circ}$, although the former do not cover as large an energy range as the latter. Oxygen-16 ions were accelerated in the ORNL Tandem Van de Graaff accelerator. The energy resolution of the beam, determined by the geometry of the $90^{\circ}$ analyzing magnet, was 8 kev.

Alpha-particle groups leaving $\mathrm{Mg}^{24}$ in its ground state and first six excited states were identified; the detectors were silicon surface barriers. The variation of center-of-mass angle with bombarding energy amounted to about $0.2^{\circ}$. The second and third excited states differ by only $0.11 \mathrm{Mev}$ and could not be resolved because of the kinematic variation of particle energy over the $3.6^{\circ}$ laboratory ( $5.2^{\circ}$ c.m.) angular acceptance of the counter. A better-than-average pulse-height spectrum is shown in Fig. 1.17.

The target was an $11 \pm 3 \mu \mathrm{g} / \mathrm{cm}^{2}$ carbon film. The most serious experimental difficulty was the carbon buildup on the target, about $0.6 \mu \mathrm{g} \mathrm{cm}^{-2}$ $\mathrm{hr}^{-1}$. A second silicon counter, fixed at about $62^{\circ}$ c.m., detected elastically scattered $\mathrm{O}^{16}$ ions and was used to monitor the carbon buildup. The alpha yields were normalized to the elastic scattering. A portion of the target was used until the carbon thickness reached $15 \mu \mathrm{g} / \mathrm{cm}^{2}$; the target

${ }^{26} \mathrm{~L}$. Colli el al., Pbys. Letters 1, 120 (1962); 2, 12 (1962).

${ }^{27}$ C. P. Browne, Phys. Rev. 114, 807 (1959).

$28 \mathrm{~J}$. A. Kuehner, E. Almqvist, and D. A. Bromley, Nucl. Phys. 21, 555 (1960).

${ }^{29}$ T. Ericson, Advan. Pbys. 9, 425 (1960). was then moved so that the beam hit a fresh part. On the average the target was about 12 to $13 \mu \mathrm{g} / \mathrm{cm}^{2}$ thick, corresponding to an energy loss of about $110 \mathrm{kev}$ for the $\mathrm{O}^{16} \mathrm{ions}$, or $48 \mathrm{kev}$ c.m. The latter number is effectively the energy resolution in this experiment. In a later run, a shield at liquidnitrogen temperature was placed around the target, and no change in target thickness was observed.

Figures 1.18 and 1.19 show the excitation functions for four alpha-particle groups. The yields for the fifth and sixth excited states fluctuated in a similar way; however, the background (probably due to alpha particles from target impurities) made it impossible to obtain reliable measurements of their yields. The cross sections were calculated by comparison with the elastic counting rates, which were assumed to be due to pure Coulomb scattering. To check this assumption, we decreased the beam energy to $17.64 \mathrm{Mev}$ ( $7.65 \mathrm{Mev}$ c.m.) and measured the elastic counting rate at four angles from 62 to $92^{\circ}$. The rate had a $\csc ^{4} \theta / 2$ dependence and was also larger by a factor of $1 / E^{2}$ at $62^{\circ}$ than that for the higher-energy data.

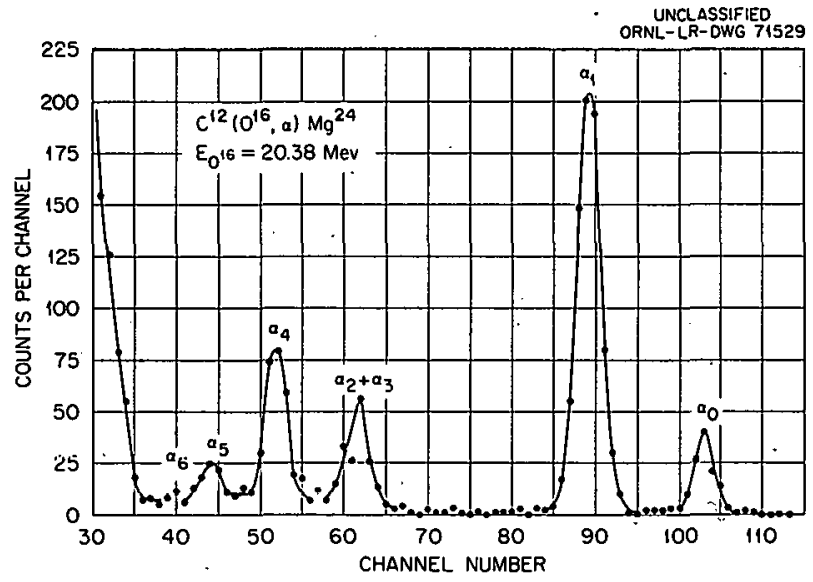

Fig. 1.17. Pulse-Height Spectrum at $20.38 \mathrm{Mev}(8.73$ Mev c.m.). Alpha-particle groups leaving $\mathrm{Mg}^{24}$ in its ground state and first six excited states are indicated. The angular acceptance of the counter was too large to resolve the second and third excited states. The sensitive depth of the surface-barrier detector was adjusted so that no protons or deuterons produced pulses above channel 35. 


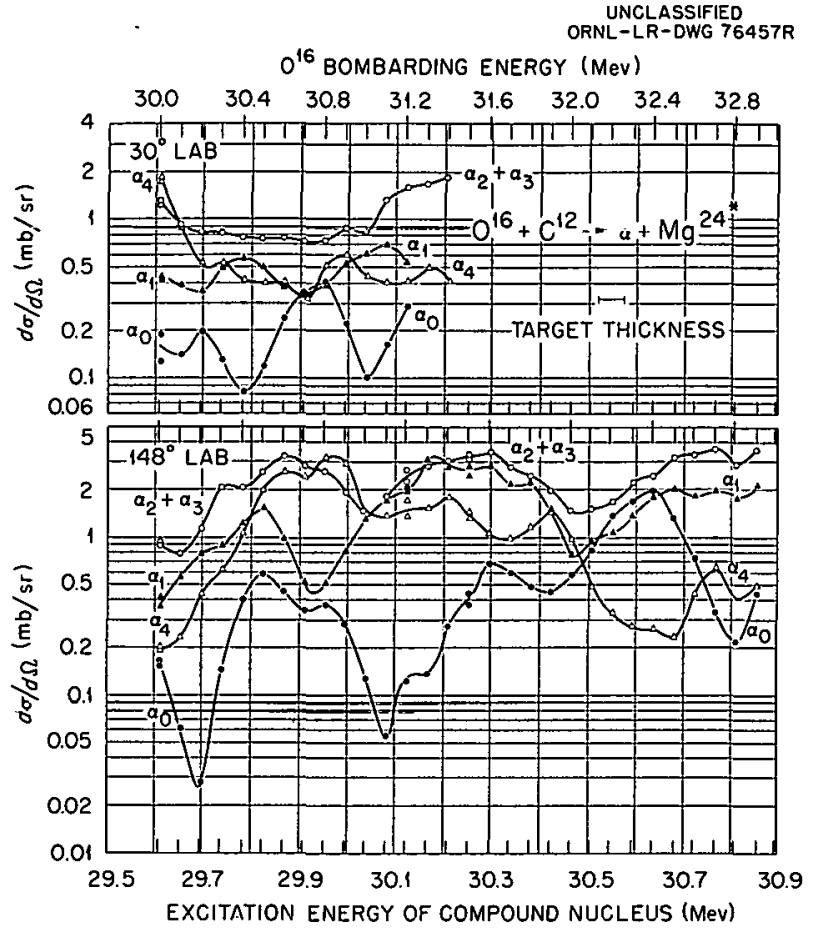

Fig. 1.18. Excitation Functions for Alpha-Particle Groups Leaving $\mathrm{Mg}^{24}$ in Its Ground State and First Four Excited States.
In the excitation functions we note first of all that peaks and valleys show up very strongly; evidently, this kind of structure is not restricted to nucleon- and deuteron-induced reactions. The most prominent peaks have widths several times the target thickness; thus the results are not affected appreciably by the experimental resolution. The full width of the peaks at half-maximum varies from $\sim 85$ to $\sim 220 \mathrm{kev}$.

Rapid variations in cross section imply the participation of long-lived intermediate states. One possible interpretation of these phenomena is based on the statistical model. Ericson has shown 29 that according to this model the fluctuations measure the width for decay of the compound nucleus, provided that compound states of similar properties are randomly distributed in energy. If compound states with large matrix elements for transitions to a particular final system are bunched together over a range of energies exceeding the width of any one level, then peaks in the excitation function will be broader than the compoundnucleus width. We conclude that for the narrowest peaks the lifetime of the compound system is no shorter than about $0.7 \times 10^{-20}$ sec.

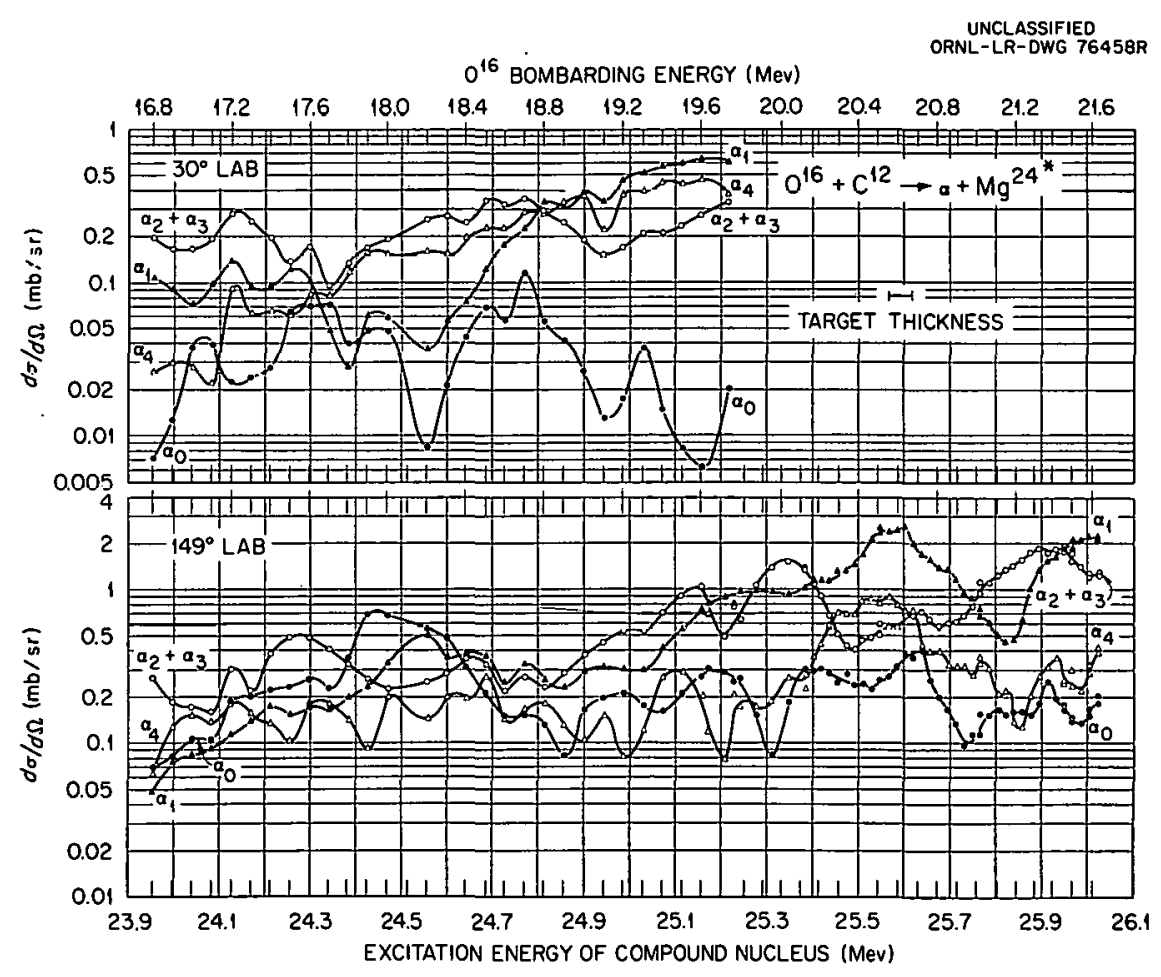

Fig. 1.19. Excitation Functions for Alpha-Particle Groups Leaving $\mathrm{Mg}^{24}$ in Its Ground State and First Four Excited Stotes. 
The data of Ogata et al. ${ }^{24}$ on $\mathrm{Al}^{27}(p, \alpha) \mathrm{Mg}^{24}$ partially overlap ours; the region from 25.1 to 25.6 Mev excitation in $\mathrm{Si}^{28}$ (14.1 to $14.5 \mathrm{Mev}$ proton energy) is common to both experiments. The structure in this energy region is less pronounced in the $(p, \alpha)$ data, which may indicate that direct interactions, which can vary only rather slowly with energy, are contributing a large portion of the $(p, a)$ cross section. This supposition is borne out by the data, since the angular distributions ${ }^{24}$ averaged over this energy region show a backward peak. This is not in conflict with the observation 23 that the angular distributions averaged from 11.0 to 14.5 Mev proton bombarding energy are symmetric about $90^{\circ}$, since the cross sections above 14.1 Mev are small and do not contribute much to the average. The fluctuations in the $\mathrm{O}^{16}+\mathrm{C}^{12}$ experiment appear equally pronounced over the entire energy region investigated; no damping at high energy is observed.'

Ericson has suggested that the average compoundnucleus width, $\Gamma$, may be determined experimentally by means of the autocorrelation function

$$
H(\epsilon)=\frac{\langle[\sigma(E+\epsilon)-\langle\sigma\rangle][\sigma(E)-\langle\sigma\rangle]\rangle}{\langle\sigma\rangle^{2}},
$$

since this function should include the Lorentz factor $\Gamma^{2} /\left(\epsilon^{2}+\Gamma^{2}\right)$. Figure 1.20 shows the auto-

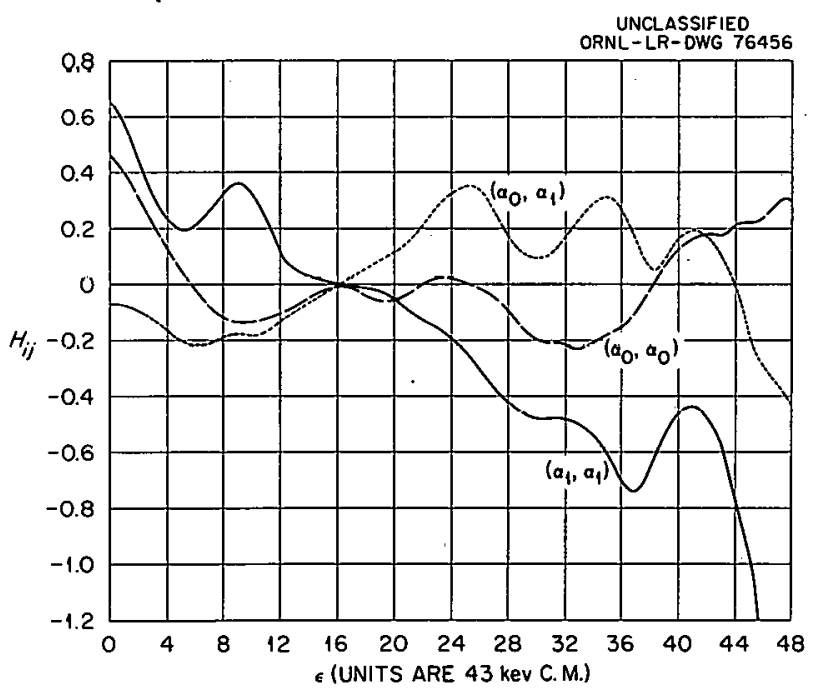

Fig. 1.20. Correlation Functions for $\alpha_{0}$ and $\alpha_{1}$ for the $150^{\circ}$ Data of Fig. 1.18. The autocorrelations are designated by $\left(a_{0}, a_{0}\right)$ and $\left(a_{1}, a_{1}\right)$; the symbol $\left(a_{0}, a_{1}\right)$ identifies the cross correlation. correlation for $a_{0}$ and $a_{1}$ for the block of data covering the widest energy range, namely, the $150^{\circ}$ data from 23.9 to $26.0 \mathrm{Mev}$. The width $\Gamma$ is about $120 \mathrm{kev}$ for each. The significance of the remaining structure in Fig. 1.20 is not clear, particularly the peak near $\epsilon=9$. For large $\epsilon$ the oscillations are probably not significant because the number of data points entering into the calculation of the averages becomes small. Similar autocorrelations have been obtained for $\left(\alpha_{2}+\alpha_{3}\right)$ and $\alpha_{4}$; these are shown in Fig. 1.21.

Figures 1.20 and 1.21 also contain cross correlations between pairs of final states to test Ericson's prediction that different final states are uncorrelated. The cross correlation for states $i$ and $j$ is defined by a symmetrized extension of $H(\epsilon)$ :

$$
\begin{aligned}
H_{i j}(\epsilon)= & \frac{1}{2} \frac{\left\langle\left[\sigma_{i}(E+\epsilon)-\left\langle\sigma_{i}\right\rangle\right]\left[\sigma_{j}(E)-\left\langle\sigma_{j}\right\rangle\right]\right\rangle}{\left\langle\sigma_{i}\right\rangle\left\langle\sigma_{j}\right\rangle} \\
& +\frac{1}{2} \frac{\left\langle\left[\sigma_{i}(E)-\left\langle\sigma_{i}\right\rangle\right]\left[\sigma_{j}(E+\epsilon)-\left\langle\sigma_{j}\right\rangle\right]\right\rangle}{\left\langle\sigma_{i}\right\rangle\left\langle\sigma_{j}\right\rangle} .
\end{aligned}
$$

It may be seen that $H_{i j}$ is satisfyingly small near $\epsilon=0$ in both Figs. 1.20 and 1.21. The behavior of the cross correlations at large $\epsilon$ is not understood, although undoubtedly some of the difficulty arises from the poor statistics alluded to previously.

In any case the remarkable difference between the autocorrelations and cross correlations supports Ericson's picture. The 120 -kev width then corresponds to a compound-nucleus lifetime of about $0.5 \times 10^{-20} \mathrm{sec}$.

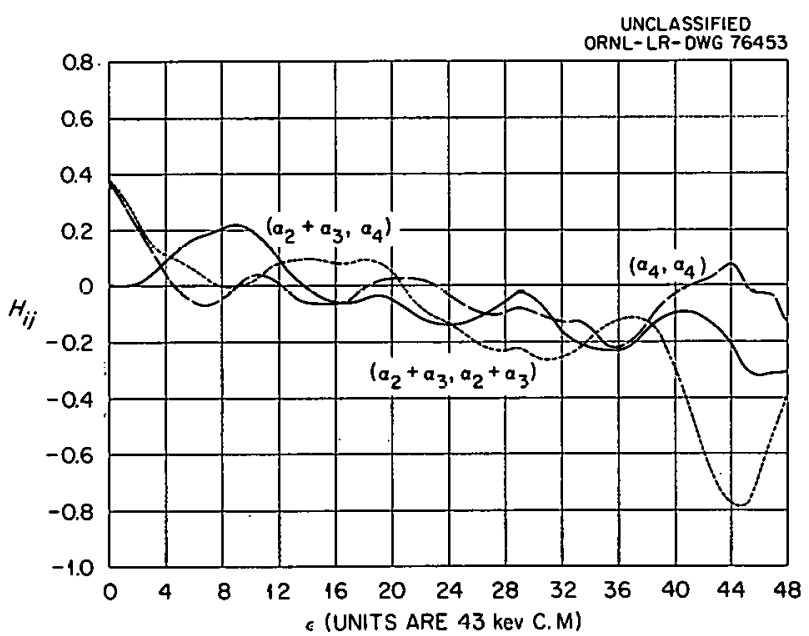

Fig. 1.21. Correlation Functions for $\left(a_{2}+a_{3}\right)$ and $\alpha_{4}$ for the $150^{\circ}$ Data of Fig. 1.18. 


\section{Physics Research with 22-Mev Protons}

\author{
C. D. Goodman \\ J. B. Ball \\ C. B. Fulmer \\ B. Harmatz
}

The nuclear physics research program at the ORNL 86-Inch Cyclotron continues to be concerned with the structure of nuclei and with nuclear reaction mechanisms. For convenience, this summary is subdivided into five parts, namely, studies of the pickup-reaction mechanism, shellmodel studies using pickup reactions, scattering and the optical model, level schemes in highly deformed nuclei, and instrumentation and techniques.

\section{STUDIES OF THE PICKUP-REACTION MECHANISM}

The experimental data on $(p, d),(p, t)$, and $(p, a)$ reactions obtained with $22-\mathrm{Mev}$ protons show that for each reaction, the cross sections for exciting different final states differ by large factors (10 to 100 ), even though the reaction $Q$ values may differ by less than $1 \mathrm{Mev}$, and the angular distributions of the emitted particles are strongly forward-peaked. These attributes of the data are characteristic of direct nuclear reactions, but attempts to pin down precisely the nature of the direct interaction bring one face to face with a knotty problem of the interrelationship between nuclear structure and the reaction mechanism. This problem is of special significance when one attempts to derive nuclearstructure information from intercomparisons of the data from the several reactions.

\footnotetext{
${ }^{1}$ ORINS Research Participant, Virginia Polytechnic Institute.

${ }^{2}$ Visiting scientist, University of Maryland.

${ }^{3}$ ORINS Fellow, University of Texas.
}
E. L. Olson
J. C. Stoltzfus ${ }^{1}$
H. Holmgren ${ }^{2}$
R. F. Sweet ${ }^{3}$

Formally, the cross section for a pickup reaction may be written as the product of two factors, $\sigma=\sigma_{r} s$. The $\sigma_{r}$ represents the cross section for picking up a nucleon occupying a state of a given binding energy and angular momentum, and $S$ represents the probability that a nucleon will be found in that state.

In an effort to study $\sigma_{r}$ for several different kinds of pickup reactions, situations were chosen for which the estimation of $S$ is particularly simple, that is, situations in which $S$ is expected to be unity.

Consider the reaction $\mathrm{Zr}^{91}(p, d) \mathrm{Zr}^{90}$ leading to the ground state of $\mathrm{Zr}^{90}$. To the accuracy with which $\mathrm{Zr}^{91}$ may be considered as $\mathrm{Zr}^{90}$ plus one $d_{S / 2}$ neutron, $S=1$ for this reaction. Likewise, insofar as $\mathrm{Zr}^{92}$ may be considered as $\mathrm{Zr}^{90}$ plus a pair of $d_{S / 2}$ neutrons, $S=1$ for $\mathrm{Zr}_{\mathrm{r}}^{92}(p, t) \mathrm{Zr}_{\mathrm{r}}{ }^{90}$ leading to the ground state of $\mathrm{Zr}_{\mathrm{r}}^{90}$. Niobium-93 may be considered as $\mathrm{Zr}^{90}$ plus a pair of neutrons and a proton. Thus, for the reaction $\mathrm{Nb}^{93}(p, a) \mathrm{Zr}_{\mathrm{r}}^{90}$ leading to the ground state of $\mathrm{Zr}^{90}, S$ is expected to be unity except for the modification brought about by the fact that the proton is in a different shell from the neutrons.

The experimental data are shown in Fig. 2.1. These data should serve to evaluate the validity of theoretical calculations of the cross sections by the use of distorted-wave methods described in Sec 3.

Similar data have been obtained for $(p, d),(p, t)$, and $(p, \alpha)$ leading to $\mathrm{Fe}^{56}$ as the final nucleus. It was expected that the approximation that the targets $\mathrm{Fe}^{57}, \mathrm{Fe}^{58}$, and $\mathrm{Co}^{59}$ look like $\mathrm{Fe}^{56}$ plus one neutron, two neutrons, and two neutrons and a proton would not be valid in the same way as it 


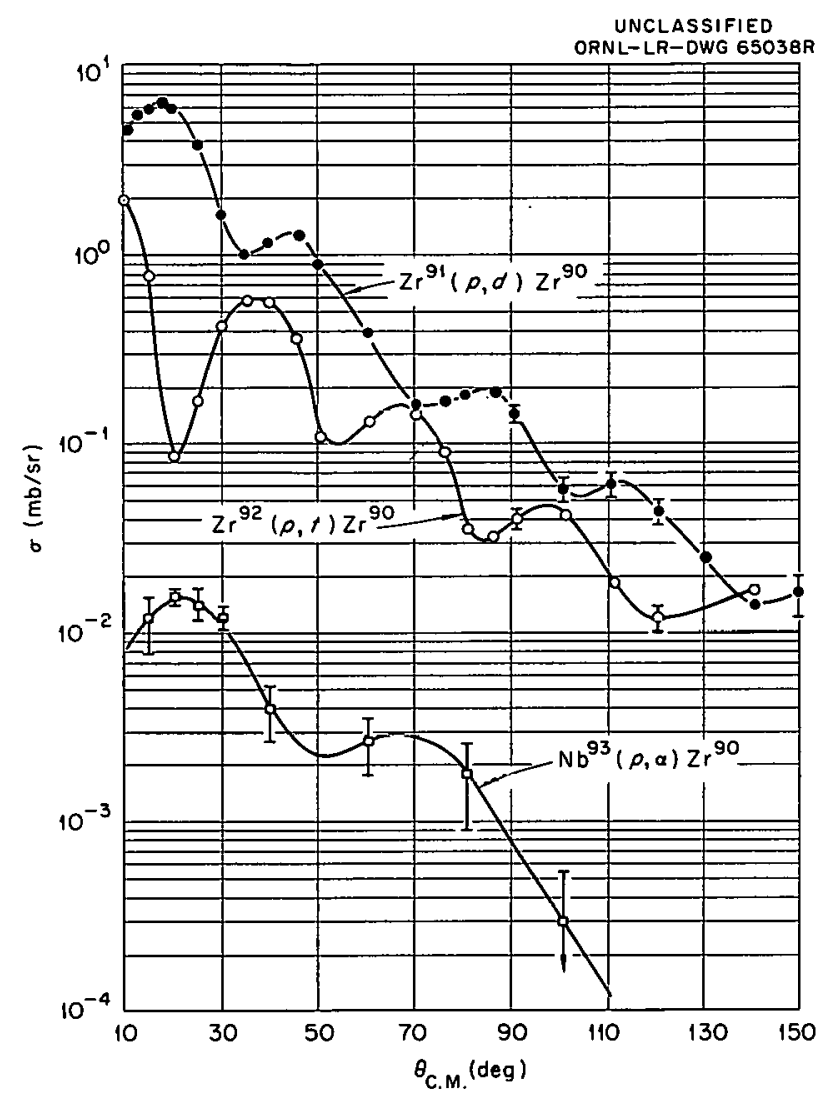

Fig. 2.1. Angular Distributions of Deuterons from $\mathrm{Zr}^{91}$, Tritons from $\mathrm{Zr}^{92}$, and Alpha Particles from $\mathrm{Nb}^{93}$ Leading to the Ground State of $Z_{r}{ }^{90}$.

was for the zirconium cases. The interpretation is more complicated due to the presence of partially filled levels in the final nucleus. In the iron case a picture similar to that used to describe the zirconium data can be retained if the $\mathrm{Fe}^{56}$ "core" is assumed to be nonspherical. The deformation explains the strong excitation of the $2+(0.85 \mathrm{Mev})$ and $4+(2.1 \mathrm{Mev})$ states in $\mathrm{Fe}^{57}(p, d) \mathrm{Fe}^{56}$ and the relatively weak excitation of the same states in $\mathrm{Fe}^{58}(p, t) \mathrm{Fe}^{56}$. The $\mathrm{Co}^{59}(p, \alpha) \mathrm{Fe}^{56}$ reaction is even more complicated, apparently involving a spherical target and a deformed final state. The data are shown in Figs. 2.2-2.4.

\section{SHELL-MODEL STUDIES USING PICKUP REACTIONS}

In the simplest cases of pickup reactions, the interaction between the picked up nucleons and the rest of the nucleus is assumed to be completely described by the shell-model potential. This potential

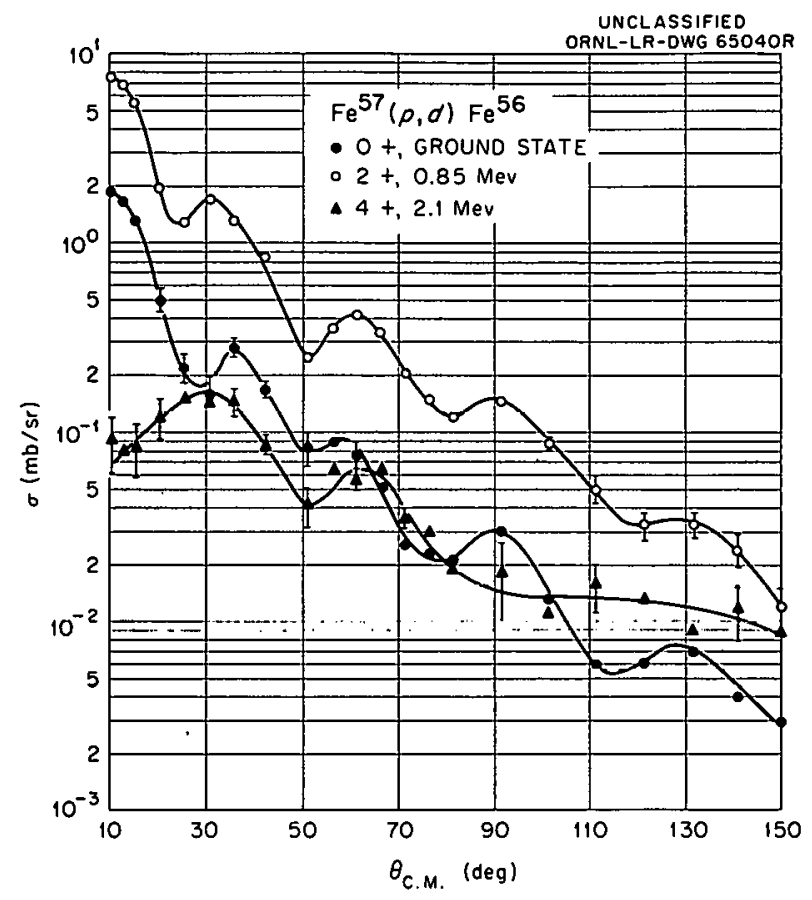

Fig. 2.2 Angular Distributons of Deuterons, from Bombardment of $\mathrm{Fe}^{57}$ with 22.3. Mev Protons, Leading to the Three Lowest States of $\mathrm{Fe}^{56}$.

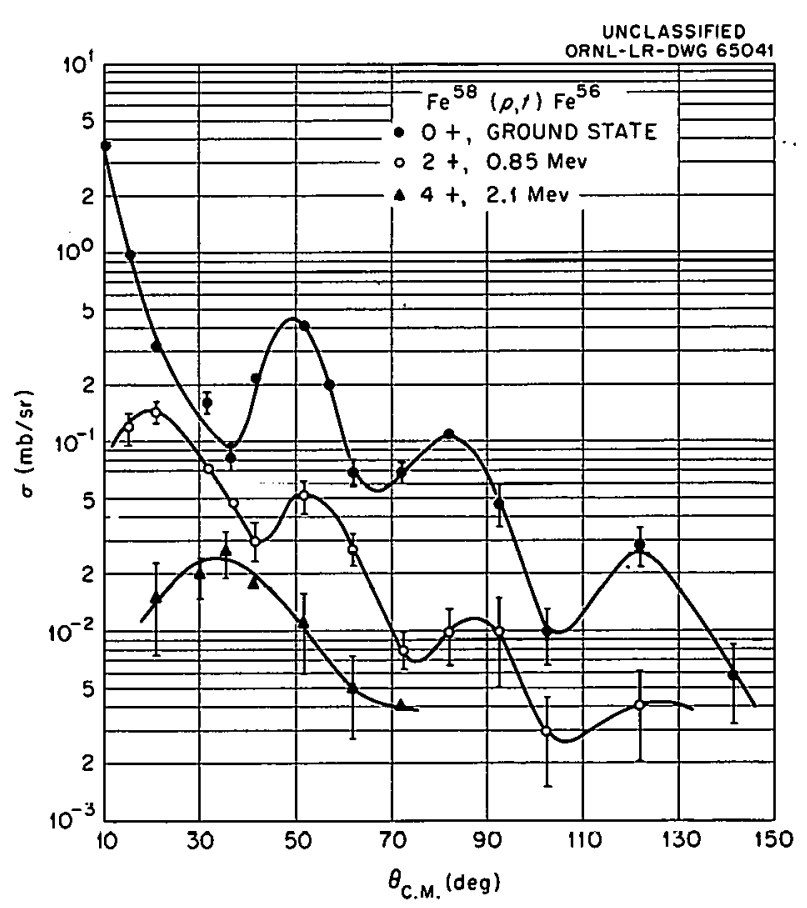

Fig. 2.3. Angular Distributions of Tritons, from Bombardment of $\mathrm{Fe}^{58}$ with 22.3-Mev Protons, Leading to the Three Lowest States of $\mathrm{Fe}^{56}$. 


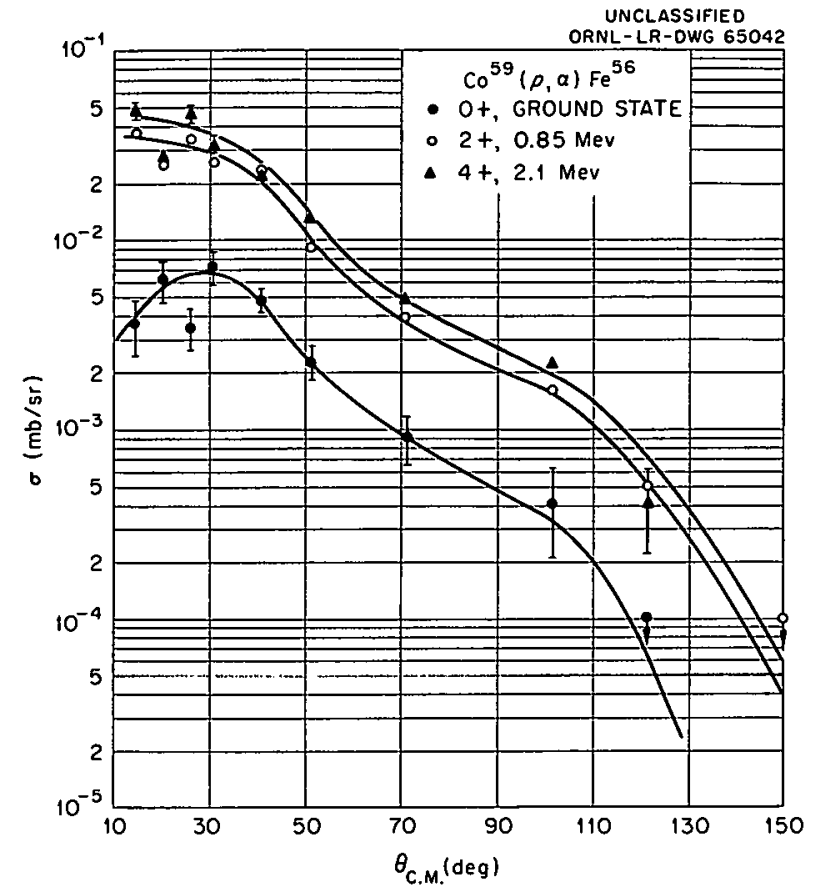

Fig. 2.4. Angular Distributions of Alpha Particles, from Bombardment of $\mathrm{Co}^{59}$ with 22.3-Mev Protons, Leading to the Three Lowest States of $\mathrm{Fe}^{56}$.

is assumed to be the same for the target and the final nucleus. The difference bctween the target and the final nucleus is simply that the bound state occupied in the target by the picked up nucleon is vacant in the final nucleus. Thus the pickup of a given nucleon from the target leads to a definite energy level of the final nucleus characterized by the state in which the vacancy has been created.

This picture, oversimplified as it is, works remarkably well near closed shells and is usable with slight modification for a closed shell plus a few nucleons. Consider $\mathrm{Nb}^{93}(p, d) \mathrm{Nb}^{92}$. The pickup of one of the $d_{5 / 2}$ neutrons would be expected to leave $\mathrm{Nb}^{92}$ in its ground state. The fact that the $(p, d)$ spectra from $\mathrm{Nb}^{93}(p, d) \mathrm{Nb}^{92}$ and $\mathrm{Zr}^{92}(p, d) \mathrm{Zr}^{91}$ are quite similar indicares that the influence of the odd $g_{9 / 2}$ proton on the neutronbound states is small. However, the influence is not unobservable, and there is a wealth of information to be obtained by looking at the $d_{5 / 2}$ pickup peak with good resolution. As shown in Fig. 2.5, the peak does not consist of a single state but of several states spread over an energy interval of about $450 \mathrm{kev}$. This is an instance where the simple model works well enough to preserve the characterization of the neutron-bound state as the $2 d_{5 / 2}$ shell-model level and of the proton-bound state as the $1 g_{9 / 2}$ level. The several observed energy levels in $\mathrm{Nb}^{92}$ evidently represent the six possible angular momentum couplings of $g_{9 / 2}$ and $d_{5 / 2}$. The exact sequence and spacing are dependent on the force between the proton and neutron and could be used as a means of determining the proton-neutron residual force. The pickup reaction is used to identify those states which can be interpreted as resulting from this type of interaction. The band of states is distinguished by its angular distribution. Within the band the intensities should be proportional to $2 I+1$, where $I$ is the spin of the state. Unfortunately, the six states in $\mathrm{Nb}^{92}$ cannot be resolved with the present apparatus; new instruments, described below, will improve the resolution.

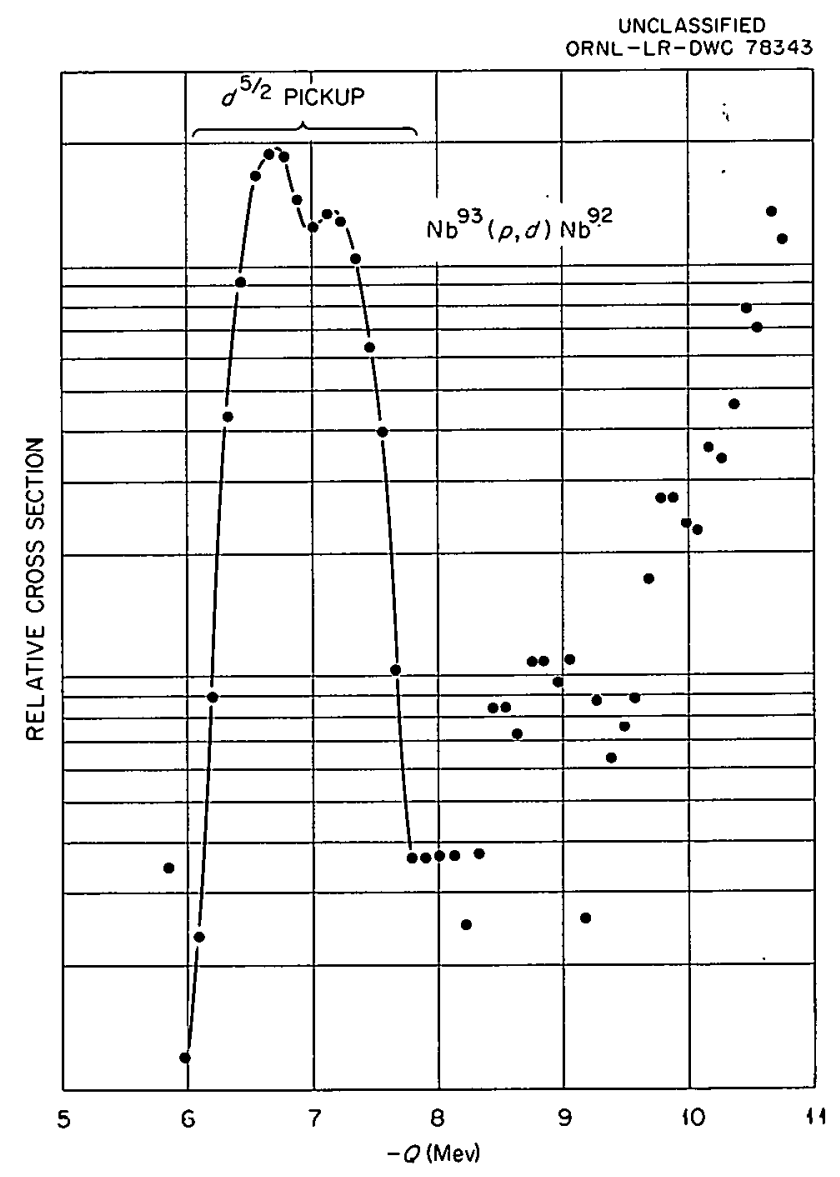

Fig. 25. Deuteron Spectrum from the Reaction $\mathrm{Nb}^{93}$ $(p, d) \mathrm{Nb}^{92}$. Note that the $d_{5 / 2}$ pickup group is brood and shows some structure. 
A similar set of states should occur in $\mathrm{Zr}^{92}$ for two neutrons in the $d_{5 / 2}$ shell, except that the Pauli principle excludes three of the six states and only the $0_{+}, 2+$, and $4+$ states are permissible. These have been studied by the reaction $Z_{\mathbf{r}}{ }^{94}(p, t) Z_{\mathbf{r}}{ }^{92}$. The interaction between two $d_{s / 2}$ neutrons is stronger than that between a $d_{5 / 2}$ neutron and a $g_{9 / 2}$ proton, and the splitting is sufficient to be resolved. The data led to the following interpretation: The $0_{+}$state is the ground state, the $2+$ state is at $0.9 \mathrm{Mev}$, and the $4+$ state is at $1.5 \mathrm{Mev}$. A similar set of states in $\mathrm{Zr}_{\mathbf{r}}{ }^{94}$ has been found by the reaction $\mathrm{Zr}^{96}(p, t) \mathrm{Zr}^{94}$. The differential cross sections are shown in Fig. 2.6.

In $Z_{r}{ }^{90}(p, d) Z_{r}{ }^{89}$ one of the observed states $(0.6$ $\mathrm{Mev}$ ) is interpreted as resulting from the pickup of a $p_{1 / 2}$ neutron (see Fig. 2.7). This state should have the shell-model configuration: two $p_{1 / 2}$ protons, one $p_{1 / 2}$ neutron, and ten $g_{9 / 2}$ neutrons. The ground state of $\mathrm{Y}^{89}$ has the configuration: one $p_{1 / 2}$ proton, two $p_{1 / 2}$ neutrons, and ten $g_{9 / 2}$ neutrons. The $0.6-\mathrm{Mev}$ state of $\mathrm{Zr}^{89}$ and the ground state of $\mathrm{Y}^{89}$ thus have a simple relationship with each other through the substitution of a proton for a neutron. In the reaction $\mathrm{Y}^{89}(p, n) \mathrm{Zr}^{89}$ a

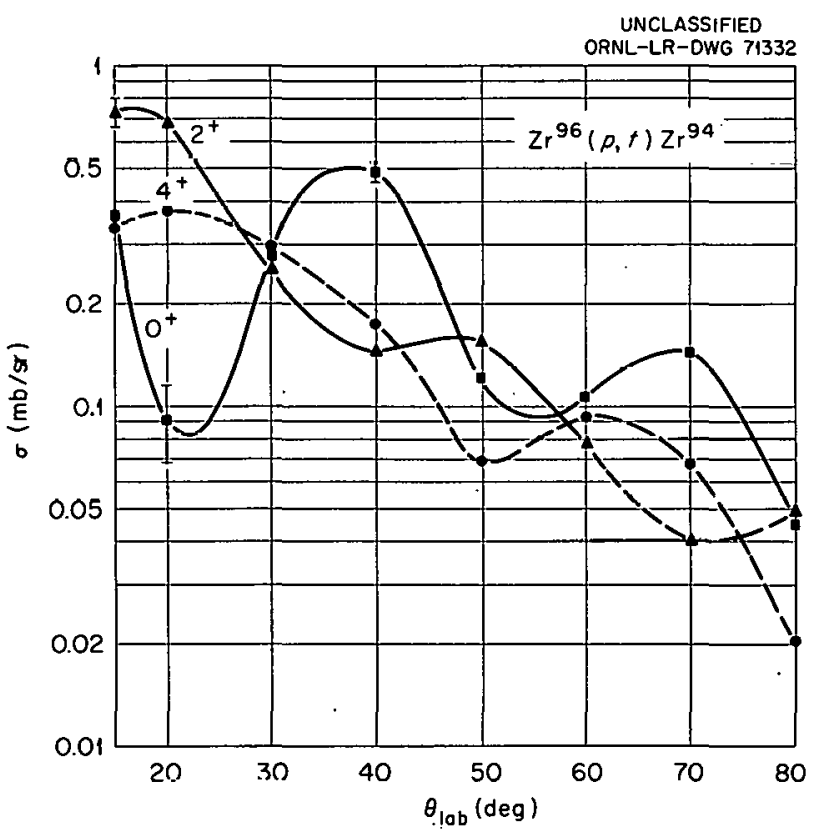

Fig. 2.6. Differential Cross Sections for the Three States Excited by Pickup of a. Pair of $d_{5 / 2}$ Neutrons from $\mathrm{Zr}^{96}$.

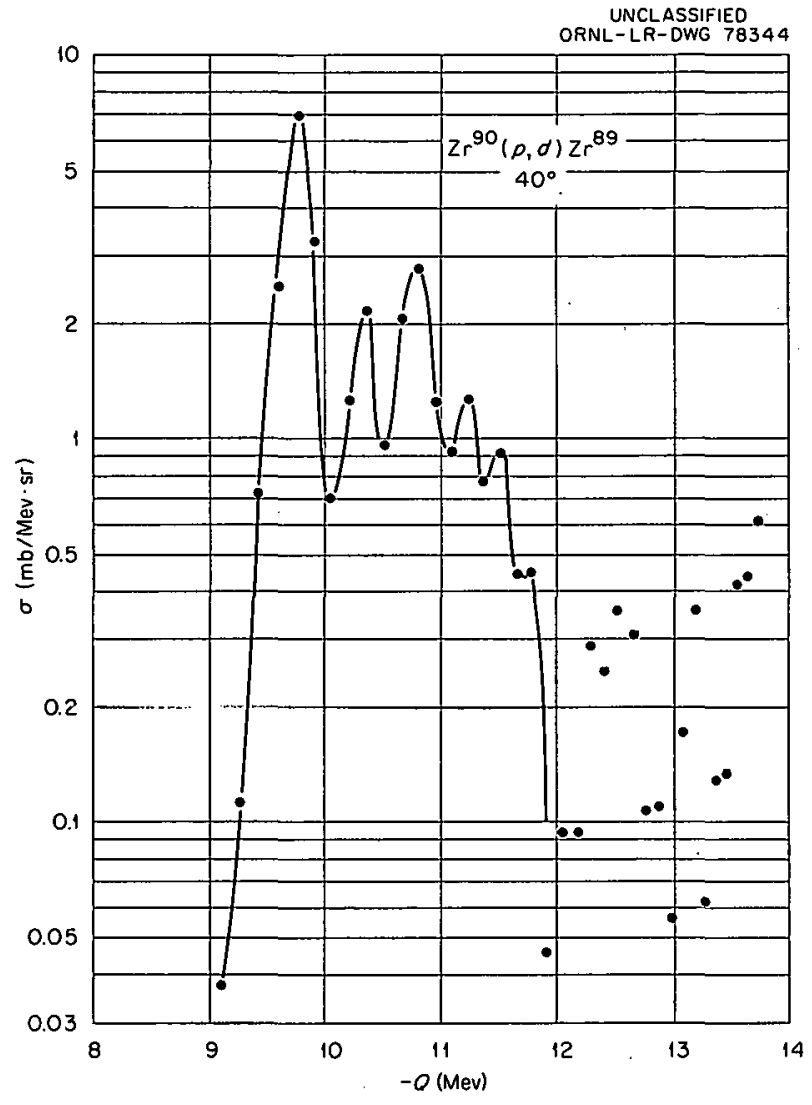

Fig. 2.7. Deuteron Spectrum from the Reaction $Z_{r}{ }^{90}$ $(p, d) \mathrm{Zr}^{89}$. The peak at $Q=-9.8$ is the ground-state group.

state is found which is interpreted as the result of the substitution of a proton for a neutron. ${ }^{4}$ The state found by the $(p, n)$ reaction is at an excitation of $8 \mathrm{Mev}$. In considering the relationship of the 0.6Mev state to the $8-\mathrm{Mev}$ state, it is useful to consider is obaric spin. In the reaction $\mathrm{Y}^{89}(p, n) \mathrm{Zr}_{\mathbf{r}}{ }^{89}$, initially $T_{z}=1 \frac{1}{2}$ and $T=1 \frac{1}{2}$, presumably. Since the $(p, n)$ reaction does not change the total isobaric spin, the final state must be $T_{z}=9 / 2, T=1 \frac{1}{2}$. In the $\mathrm{Zr}^{90}(p, d) \mathrm{Zr}^{89}$ reaction, $T_{z}=10 / 2$ and $T=10 / 2$, initially; $T_{z}=9 / 2, T=9 / 2$, as well as $T_{z}=\frac{9}{2}$, $T=1 \frac{1}{2}$, is a permissible final state. The $T=$ $9 / 2$ state is favored by a factor of 10 in intensity from isobaric-spin considerations alone. It has been suggested ${ }^{5}$ that the energy difference between

\footnotetext{
${ }^{4}$ J. D. Anderson, C. Wong, and J. W. McClure, Phys. Rev. 126, 2170 (1962).
}

$5 \mathrm{M}$. H. Macfarlane, private communication. 
the two states represents an isobaric-spin splitting of the type described theoretically. ${ }^{6}$

Nuclear-structure data have been obtained also from $\mathrm{F}^{19}(p, t) \mathrm{F}^{17}$ and $\mathrm{F}^{19}(p, a) \mathrm{O}^{16}$ : It is interesting to note that $\mathrm{F}^{19}(p, t) \mathrm{F}^{17}$ excites both the $5 / 2+$ ground state and the $1 / 2+$ state at $0.5 \mathrm{Mev}$ quite strongly (see Fig. 2.8). This at once indicates that the odd proton in $\mathrm{F}^{19}$ cannot be pictured as occupying a definite $j-j$ coupling shell-model state.

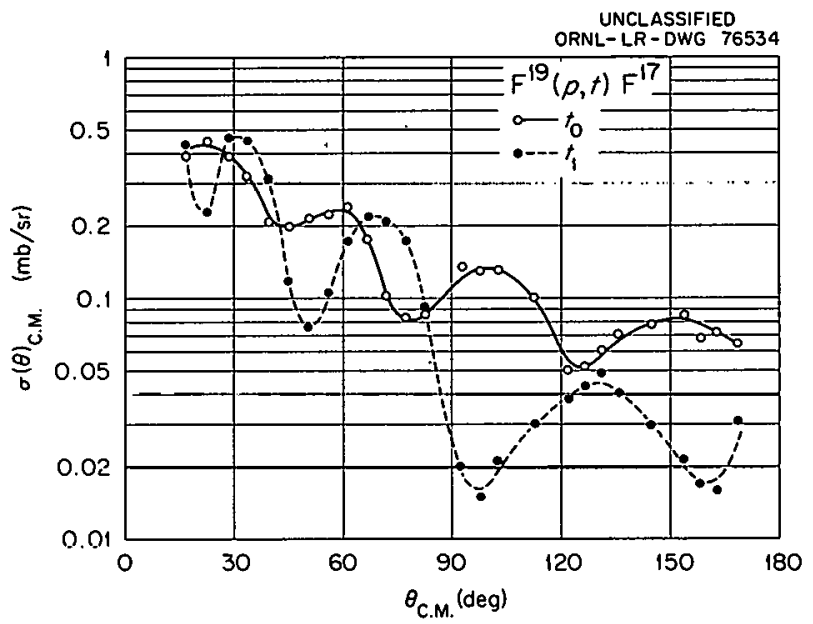

Fig. 2.8. Differential Cross Section for Excitation of the Ground State ( $t_{0}$ Group) and 0.5 -Mer State ( $t_{1}$ Group) in the Reaction $\mathrm{F}^{19}(p, t) \mathrm{F}^{17}$.

\section{SCATTERING AND THE OPTICAL MODEL}

Studies of elastic scattering to explore the dependence of the optical-model well depth on the nuclear-symmetry parameter $(N-Z) / A$ were continued. The new data include scattering of protons from the zirconium isotopes, done at $22.5 \mathrm{Mev}$ with the ORNL 86-Inch Cyclotron, and scattering of protons from the isobar pairs $\mathrm{Ni}^{64}-\mathrm{Zn}^{64}$ and $\mathrm{Fe}^{58}-\mathrm{Ni}^{58}$ at 9.6 and $11.7 \mathrm{Mev}$, done in collaboration with $J$. Benveniste and $A$. C. Mitchell on the cyclotron at the Lawrence Radiation Laboratory, Livermore, California.

The data on $\mathrm{Zr}^{90}, \mathrm{Zr}^{91}, \mathrm{Zr}^{92}, \mathrm{Zr}^{94}$, and $\mathrm{Zr}^{96}$, studied over the angular range of 17 to $159^{\circ}$, show

${ }^{6} \mathrm{~J}$. B. French and M. H. Macfarlane, Nucl. Phys. 26, 168 (1961). a smooth shift of the positions of the maxima and minima toward smaller angles for the heavier isotopes (see Fig. 2.9). This shift is larger than would be expected simply from the mass dependence and is qualitatively in agreement with an $(N-Z) / A$ dependence of the real potential well depth.

The work on $\mathrm{Ni}^{64}$ and $\mathrm{Zn}^{64}$ was previously attempted at the ORNL 86-Inch Cyclotron. At 22 Mev an angular shift was not observed. The energy was reduced by degrading with absorbers, but this technique introduced an intolerable energy spread. The experiments were then undertaken at the Livermore variable-energy cyclotron at 9.6 and $11.7 \mathrm{Mev}$, with good resolution. A shift in the angular positions of the maxima and minima was observed between the two isobars; the $\mathrm{Ni}^{64}$ pattern occurred at a slightly smaller angle than the $\mathrm{Zn}^{64}$ pattern.

A complication in the interpretation of the $A=64$ data arises because $\mathrm{Zn}^{64}$ has a larger deformation

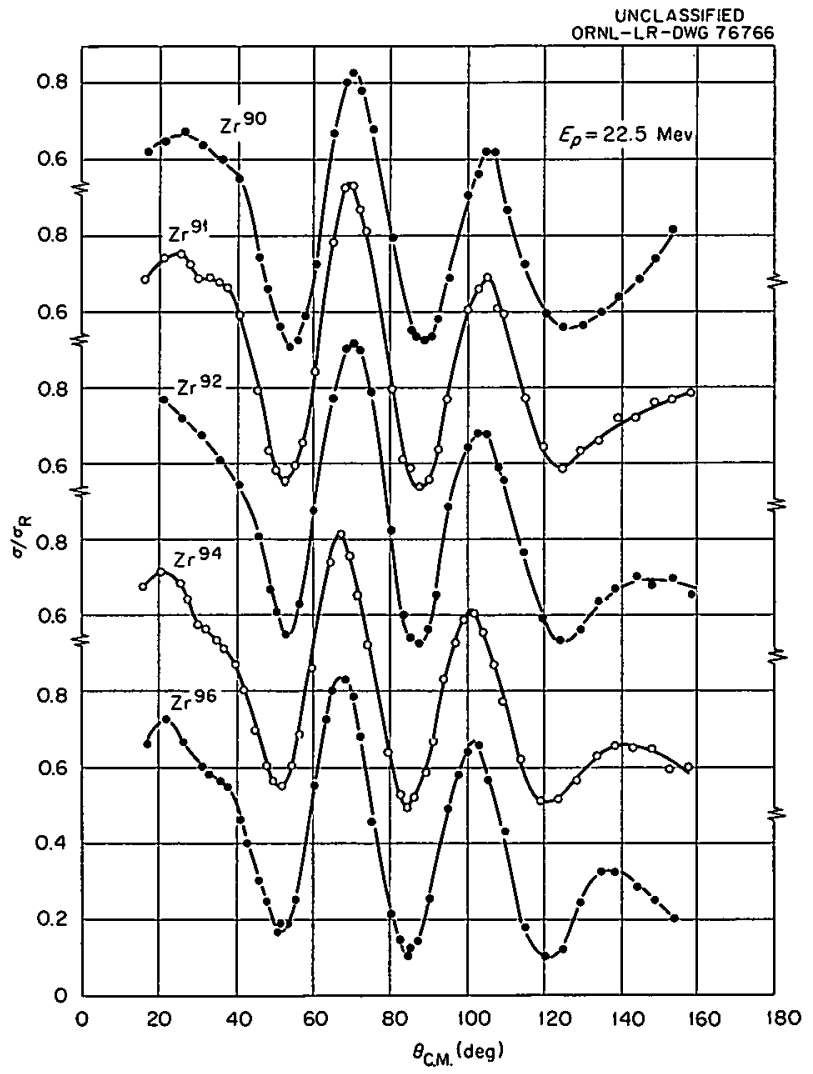

Fig. 29. Elastic Scattering of Protons from Zirconium Isotopes. 
than $\mathrm{Ni}^{64}$; it is possible that any observed angular shift might be attributed to the difference in deformation rather than to a difference in nuclearpotential well depth. To clarify this point, a second isobaric pair, $\mathrm{Fe}^{58}-\mathrm{Ni}^{58}$, was studied. The $\mathrm{Fe}^{58}$ has the larger deformation and the larger $(N-Z) / A$ of the $A=58$ pair; $\mathrm{Ni}^{64}$ has the smaller deformation and the larger $(N-Z) / A$. The angular shift is toward smaller angles for the larger $(N-Z) / A$ in both cases. This is consistent with a dependence of the real potential well depth on $(N-Z) / A$.

This experiment also resulted in the measurement of the excitation energies of 10 levels in $\mathrm{Ni}^{64}$, only 2 of which were previously measured, and of 14 levels in $\mathrm{Zn}^{64}$, some of which were not previously reported.

\section{SYSTEMATIC PROPERTIES OF NUCLEAR LEVELS IN DEFORMED ODD-A NUCLEI}

New and more precise data were obtained ${ }^{7}$ from internal-conversion electron spectra for odd- $A$ nuclei in the deformed region $N=89$ through $N=$ 113. The proposed energy levels have been characterized by excitation energy (in kev), spin and parity, and assignments of the asymptotic-limit quantum numbers of the Nilsson wave functions. ${ }^{8}$ The positions and movements of the intrinsic states and the properties of their rotational structures were followed through sequences of oddneutron nuclei. It was observed that rotational configurations are more unusual at the extreme ends of the deformed region.

The systematic behavior of the parameters which characterize rotational spectra is illustrated in Table 2.1. The last column lists $M 1 / E 2$ mixtures (of photon intensities) for the first rotational excitation in a number of $K=5 / 2$ bands. The relative $E 2$ strengths appear to be a characteristic of the odd-particle orbital, as is observed in the close-lying $5 / 2+[642]$ and $5 / 2-[523]$ configurations. It is possible to describe a correlation of experimental moments of inertia with the asymptotic quantum-number assignments. Rotational $(\Delta I=1)$ transi-

\footnotetext{
${ }^{7}$ B. Harmatz, T. H. Handley, and J. W. Mihelich, Phys. Rev. 128, 1186 (1962).

${ }^{8} \bar{B}$. R. Mottelson and S. G. Nilsson, Kgl. Danske Videnskab. Selskab, Mat.-Fys. Skrifter i(8), 1-105 (1959).
}

tions in cascade are found to have similar $M 1 / E 2$ mixing ratios, in the absence of strong particlerotational coupling effects.

Certain regularities appear in the properties of the $K=1 / 2$ band populated in the region between $\mathrm{Er}^{163}$ and $\mathbb{W}^{181}$. The $M 1 / E 2$ mixing ratios for rotational transitions between $3 / 2 \rightarrow 1 / 2$ states are low $(1.6 \pm 0.5)$ except for the case of $\mathrm{Er}^{167}$, where $M 1 / E 2=7$. Figure 2.10 presents the experimental decoupling factor $a$ for the $1 / 2-$ [521] configuration. The parameter $a$ corresponds to a partial decoupling of the particle motion from the rotating nucleus. A smooth variation is observed in $a$ as a function of mass number, indicating a maximum of +0.85 for $\mathrm{Yb}^{171}$. Mottelson and Nilsson ${ }^{8}$ have explicitly calculated the decoupling factor as a function of deformation, which is in agreement with experiment. Empirical data for the inertial term, $35^{2} / 2$, are ploted in Fig. 2.10 to show the relationship between the two rotational parameters.

The trends in the positions of intrinsic energy levels observed in spectra of udd-ncutron nuclei may be examined in Table 2.2. Good systematics are observed in the filling of neutron levels according to the scquence of Nilsson orbitals appropriate to prolate-spheriodal deformation. Usually, a level first appears as a high-lying state, decreases in energy with successivc ncutron numbcrs until it becomes the ground state ( $0 \mathrm{kev})$, then increases in energy again as more neutrons are added.

The energy shift of common single-particle states for successive isotones is, for 20 cases, less than $150 \mathrm{kev}$ and about $60 \mathrm{kev}$ on the average. The remaining 5 cases are distributed about the $200-k e v$ value. The energy spacing between common intrinsic states which appear in 'neighboring isotopes is found to vary from 20 to $600 \mathrm{kev}$. The direction of the energy displacement is internally consistent, with a few exceptions. There is, however, greater regularity to the energy shift when a neutron pair is added.

\section{INSTRUMENTATION AND TECHNIQUES}

The new 20,000-channel analyzer being constructed by Tullamore Electronics Corporation was tested at the plant with a gamma-gamma correlation experiment by Ýale and ORNL personnel. The performance of the analyzer was excellent. Magnetic tapes from the analyzer were processed by 
Table 2.1. Experimental Dato for Rotational Bands Assigned as $K=\frac{5}{2}$, Where $E\left(l_{0}\right)$ is the Energy for the Base State of Spin $I_{0}, 3 \hbar^{2} / d / s$ the Inertial Parameter, and $M 1 / E 2$ Ratios Are Obtained from

Conversion-E lectron L-Subshell Ratios

\begin{tabular}{|c|c|c|c|c|c|}
\hline$N$ & Nucleus & $\begin{array}{l}\text { Assigned Orbital } \\
\qquad K \pi\left[N n_{z} \Lambda\right]^{a}\end{array}$ & $\begin{array}{l}E\left(I_{0}\right) \\
(\mathrm{kev})\end{array}$ & $\begin{array}{l}3 \hbar^{2} / d \\
(\mathrm{kev})\end{array}$ & $\begin{array}{c}I_{0}+1 \rightarrow I_{0} \\
(M 1 / E 2)\end{array}$ \\
\hline 89 & ${ }_{64} \mathrm{Gd}^{153}$ & $s / 2+[642]$ & 129.0 & 46.5 & \\
\hline 93 & ${ }_{64} \mathrm{Gd}^{157}$ & $5 / 2+[642]$ & 64.0 & 44.4 & 34 \\
\hline 95 & ${ }_{66} \mathrm{Dy}^{161}$ & $\frac{5}{2}+[642]$ & 0 & 37.5 & 24 \\
\hline 95 & ${ }_{66} \mathrm{Dy}^{161}$ & $\frac{5}{2}-[523]$ & 25.6 & 66.9 & 0.9 \\
\hline 95 & ${ }_{68} \mathrm{Er}^{163}$ & $\frac{5}{2}-[523]$ & 0 & 72.0 & 0.12 \\
\hline 97 & ${ }_{66} \mathrm{Dyy}^{163}$ & $5 / 2-[523]$ & 0 & 63 & \\
\hline 97 & ${ }_{68} \mathrm{Er}^{165}$ & $5 / 2-[523]$ & 0 & 66.2 & Pure E2 \\
\hline 99 & ${ }_{70} \mathrm{Yb}^{169}$ & $5 / 2-[523]$ & 570.5 & 66.8 & \\
\hline 99 & ${ }_{68} E_{r^{167}}$ & $5 / 2-[523]$ & 585.4 & 71.5 & $\sim 7$ \\
\hline 99 & ${ }_{70} \mathrm{Yb}^{169}$ & $\frac{5}{2}-[512]$ & 191.4 & 75.4 & 15 \\
\hline 101 & ${ }_{70} \mathrm{Yb}^{171}$ & $5 / 2-[512]$ & 122.4 & 73.5 & \\
\hline 101 & ${ }_{72} \mathrm{Hf}^{173}$ & $5 / 2-[512]$ & 107.2 & 77.6 & \\
\hline 103 & $70^{Y^{173}}$ & $5 / 2-[512]$ & 0 & 67.6 & 20 \\
\hline 103 & ${ }_{72} \mathrm{Hf}^{175}$ & $5 / 2-[512]$ & 0 & 70.0 & 17 \\
\hline 105 & ${ }_{72} \mathrm{Hf}^{177}$ & $5 / 2-[512]$ & 508.9 & 82.6 & \\
\hline 107 & $74^{W^{181}}$ & $5 / 2-[512]$ & 365.5 & $94: 6$ & \\
\hline
\end{tabular}

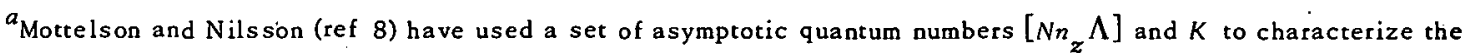
different single-particle states in the limit where the nuclear potential becomes an anisotropic harmonic oscillator.
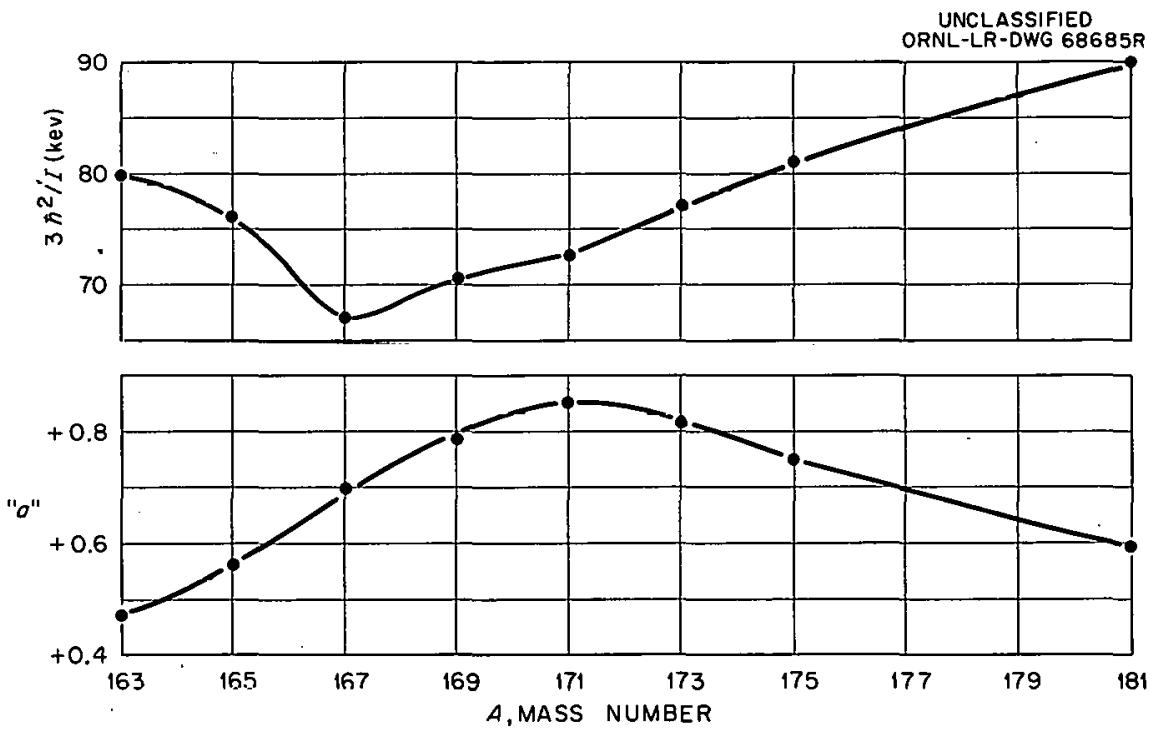

Fig. 2 10. Empirical Constants for Anomalous Rotational Sequences Based on the $1 / 2$ - [521] Configuration. 
Table 2.2. Summary of Intrinsic Excitations (in kev) Listed According to the Nilsson Orbital Description

for Odd-A Nuclei in the Region of Odd Neutron Numbers 89 to 113

Parentheses are used to designate decreasing reliability of the level assignments; double parentheses designate least certainty

\begin{tabular}{|c|c|c|c|c|c|c|c|c|c|c|c|c|c|c|c|c|}
\hline$N$ & Nucleus & $\begin{array}{c}3 / 2- \\
{[532]}\end{array}$ & $\begin{array}{c}3 / 2^{+} \\
{[402]}\end{array}$ & $\begin{array}{c}\frac{1}{2}+ \\
{[660]}\end{array}$ & $\begin{array}{c}3 / 2+ \\
{[651]}\end{array}$ & $\begin{array}{c}3 / 2- \\
{[521]}\end{array}$ & $\begin{array}{c}5 / 2^{+} \\
{[642]}\end{array}$ & $\begin{array}{c}5 / 2- \\
{[523]}\end{array}$ & $\begin{array}{c}7 / 2^{+} \\
{[633]}\end{array}$ & $\begin{array}{c}1 / 2- \\
{[521]}\end{array}$ & $\begin{array}{c}s / 2- \\
{[s 12]}\end{array}$ & $\begin{array}{c}7,2- \\
{[514]}\end{array}$ & $\begin{array}{c}9 / 2^{+} \\
{[624]}\end{array}$ & $\begin{array}{c}1 / 2- \\
{[510]}\end{array}$ & $\begin{array}{c}3 / 2- \\
{[512]}\end{array}$ & $\begin{array}{c}7 \\
2- \\
{[503]}\end{array}$ \\
\hline 89 & $\mathrm{Sm}^{151}$ & (344) & $((104))$ & (4) & (0) & $(((167))$ & $((209))$ & & & & & & & & 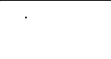 & \\
\hline 89 & $\mathrm{Gd}^{153}$ & (212) & $(109)$ & & (0) & $((303))$ & (129) & & & & & & & & & \\
\hline 91 & $\mathrm{Sm}^{153}$ & & & & & 0 & & & & & & & & & & \\
\hline 91 & $\mathrm{Gd}^{155}$ & $((326))$ & $((367))$ & $(247)$ & (86) & 0 & $(105)$ & $((286))$ & & & & & & & & \\
\hline 93 & $\mathrm{Gd}^{157}$ & & & & & 0 & $(64)$ & & & & & & & & & \\
\hline 95 & $\mathrm{Gd}^{159}$ & & & & & 0 & & & & & & & & & & \\
\hline 95 & Dy ${ }^{161}$ & & & & & 74 & 0 & 25 & & & & & & & & \\
\hline 95 & $\mathrm{Er}^{163}$ & & & & $((415))$ & $(104)$ & $(((22)))$ & (0) & & $(345)$ & & & & & & \\
\hline 97 & Dy ${ }^{163}$ & & & & & & & 0 & & & & & & & & \\
\hline 97 & $E_{r}{ }^{165}$ & & $((1427))$ & & $((853))$ & $(242)$ & $(47)$ & 0 & $((117))$ & (297) & $(608)$ & & & & & \\
\hline 97 & $\mathrm{Yb}^{167}$ & & & & & $((239))$ & (29) & $(0)$ & & & & & & & & \\
\hline 99 & $D y^{163}$ & : & & & & & & & $(0)$ & $(108)$ & & & & & & . \\
\hline 99 & $\mathrm{Er}^{167}$ & & & & & & & $((585))$ & 0 & 207 & $((347))$ & & & & & \\
\hline 99 & $\mathrm{Yb}^{169}$ & & & & & & & $(5710)$ & 0 & $(24)$ & $(191)$ & $:(962))$ & & & & $((1465))$ \\
\hline 101 & $\mathrm{Yb}^{171}$ & & & & & & & & (95) & 0 & 122 & (835) & $((936))$ & & & \\
\hline 101 & $\mathrm{Hf}^{173}$ & & & & & & & & & 0 & $((107))$ & & & & & \\
\hline 103 & $\mathrm{Yb}^{173}$ & & & & & & & & $35 i$ & & 0 & 636 & & & & \\
\hline 103 & $\mathrm{Hf}^{175}$ & & & & & & & & (207) & 125 & 0 & (348) & & & & $((1045))$ \\
\hline 105 & $\mathrm{Yb}^{175}$ & & & & & & & & & & & $(0)$ & & $((455))$ & & \\
\hline 105 & $\mathrm{Hf}^{177}$ & & & & & & & & $(746)$ & & $(508)$ & 0 & 321 & & & $(1058)$ \\
\hline 105 & $w^{179}$ & & & & & & & & & & & $(0)$ & & $((221))$ & & \\
\hline 107 & $\mathrm{Yb}^{177}$ & & & & & & & & & & · & $((104))$ & $(0)$ & $((326))$ & & \\
\hline 107 & $\mathrm{Hf}^{179}$ & & & & & & & & & & & $(215)$ & 0 & $(3.75)$ & & \\
\hline 107 & $W^{181}$ & & & & & & & & $((953))$ & $((746))$ & 365 & $((408))$ & 0 & $((385))$ & $((560))$ & $((807))$ \\
\hline 107 & Os ${ }^{183}$ & & & & & & & & & & & & 0 & $(170)$ & & \\
\hline 109 & $W^{183}$ & & & & & & & & & & & & $((309))$ & 0 & 208 & 453 \\
\hline 109 & $O s^{185}$ & & & & & & & & & & & & & 0 & 127 & $((356))$ \\
\hline 111 & $W^{185}$ & & & & & . & & & & & & & & & $(0)$ & \\
\hline 111 & $O s^{187}$ & & & & & & & & & & & & . & 0 & (74) & \\
\hline 113 & Os ${ }^{189}$ & & & & & & & & & & & & & 36 & 0 & $((217))$ \\
\hline
\end{tabular}


computers at both Yale and Oak Ridge, and a tape written by the Oak Ridge IBM 7090 was read correctly by the analyzer. The map and contour display were judged to be easy to use and of great value in examining data, both during an experiment and after subsequent processing of the data.

A magnet system for analyzing the external beam of the ORNL 86-Inch Cyclotron and the charged particles from reactions was completed; an eight-detector scintillation-counter array is nearing completion for use in the focal plane of the magnet. This system will be used for experiments for which the resolution of the $d E / d x, E$ system is inadequate, as, for example, the shellmodel studies described above. A lithium-drifted counter was purchased for use as the $E$ counter in the $d E / d x, E$ telescope for data taking. The resolution obtained with it, shown in Fig. 2.7, is in some instances considerably better than that obtained with sodium iodide scintillation detectors. 


\title{
3. Theoretical Physics
}

\author{
R. H. Bassel \\ R. M. Drisko \\ E. C. Halbert
}

N. Austern ${ }^{1}$

G. R. Satchler ${ }^{2}$

In theoretical investigations of the mechanisms of nuclear reactions and related nuclear structure information, the elastic. scattering of complex particles was analyzed via the optical model. The applicability of the distorted-wave method to stripping reactions was investigated, and for this study a new code was written which allows for a finite range force between neutron and proton. In continuing the study of reactions involving complex particles, the inelastic scattering of carbon ions from carbon was examined.

\section{DEUTERON ELASTIC SCATTERING}

The optical-model search program written last year ${ }^{3}$ was applied in the analysis of $11-$ to $12-\mathrm{Mev}$ $(d, d)$ data ${ }^{4,5}$ for targets ranging from calcium to tin. Close fits were found in most cases. Figure 3.1 shows some of the best fits obtained. Generally speaking, the fits are not unique; often, two or more discrete sets of parameters, corresponding to distinctly different complex potentials, give good fits to the same data.

For the nuclear part of the optical potential $U(r)$, four different forms were used. These are

\footnotetext{
${ }^{1}$ University of Pittsburgh.

${ }^{2}$ Physics Division.

${ }^{3}$ R. H. Bassel, R. M. Drisko, and Edith C. Halbert, Electronuclear Div. Ann. Progr. Rept. Dec. 31, 1961, ORNL-3257, p 22.

${ }^{4}$ M. Takeda, J. Pbys. Soc. Japan 15, 557 (1960).

${ }^{5}$ G. Igo, W. Lorenz, and U. Schmidt-Rohr, Phys. Rev. 124,832 (1961).
}

described conveniently in terms of the Saxon shape,

$$
f_{\mathrm{S}}\left(r ; r_{0}, a\right) \equiv\left(1+\exp \frac{r-r_{0} A^{1 / 3}}{a}\right)^{-1}
$$

and the Gaussian shape,

$$
f_{\mathrm{G}}\left(r ;{ }_{W}, a_{W}\right) \equiv \exp -\frac{\left(r-r_{W} A^{1 / 3}\right)^{2}}{a_{W}^{2}} .
$$

The four potential forms were:

$$
\begin{aligned}
& U_{S S}(r)=-V f_{S}\left(r ; r_{0}, a\right)-i W f_{S}\left(r ; r_{0}, a\right) \\
& U_{S P}(r)=-V f_{S}\left(r ; r_{0}, a\right)-i W f_{S}\left(r ; r_{W}, a_{W}\right) \\
& U_{S D}(r)=-V f_{S}\left(r ; r_{0}, a\right)+i a_{W} \frac{\partial}{\partial r} f_{S}\left(r ; r_{W}, a_{W}\right) \\
& U_{S G}(r)=-V f_{S}\left(r ; r_{0}, a\right)-i W f_{G}\left(r ; r_{W}, a_{W}\right) .
\end{aligned}
$$

In most cases the Coulomb potential was assumed to be that between a point-charge deuteron and a uniformly charged sphere of radius $1.3 A^{1 / 3}$ fermis. Results were insensitive to details of the Coulomb assumptions.

The automatic search program used to analyze the data proceeds by the standard least-squares method of Gauss; it minimizes the quantity

$$
\begin{aligned}
\chi^{2}\left(V, r_{0}, \ldots\right) \\
\quad=\sum_{\theta} \frac{\left[\sigma_{\text {theory }}\left(\theta ; V, r_{0}, \ldots\right)-\sigma_{\text {expt }}(\theta)\right]^{2}}{[\Delta \sigma(\theta)]^{2}} .
\end{aligned}
$$

Here $V, r_{0}, \ldots$ are the adjustable optical-model parameters, and the $\Delta \sigma(\theta)$ are preassigned weighting factors. Each converged solution corresponds 


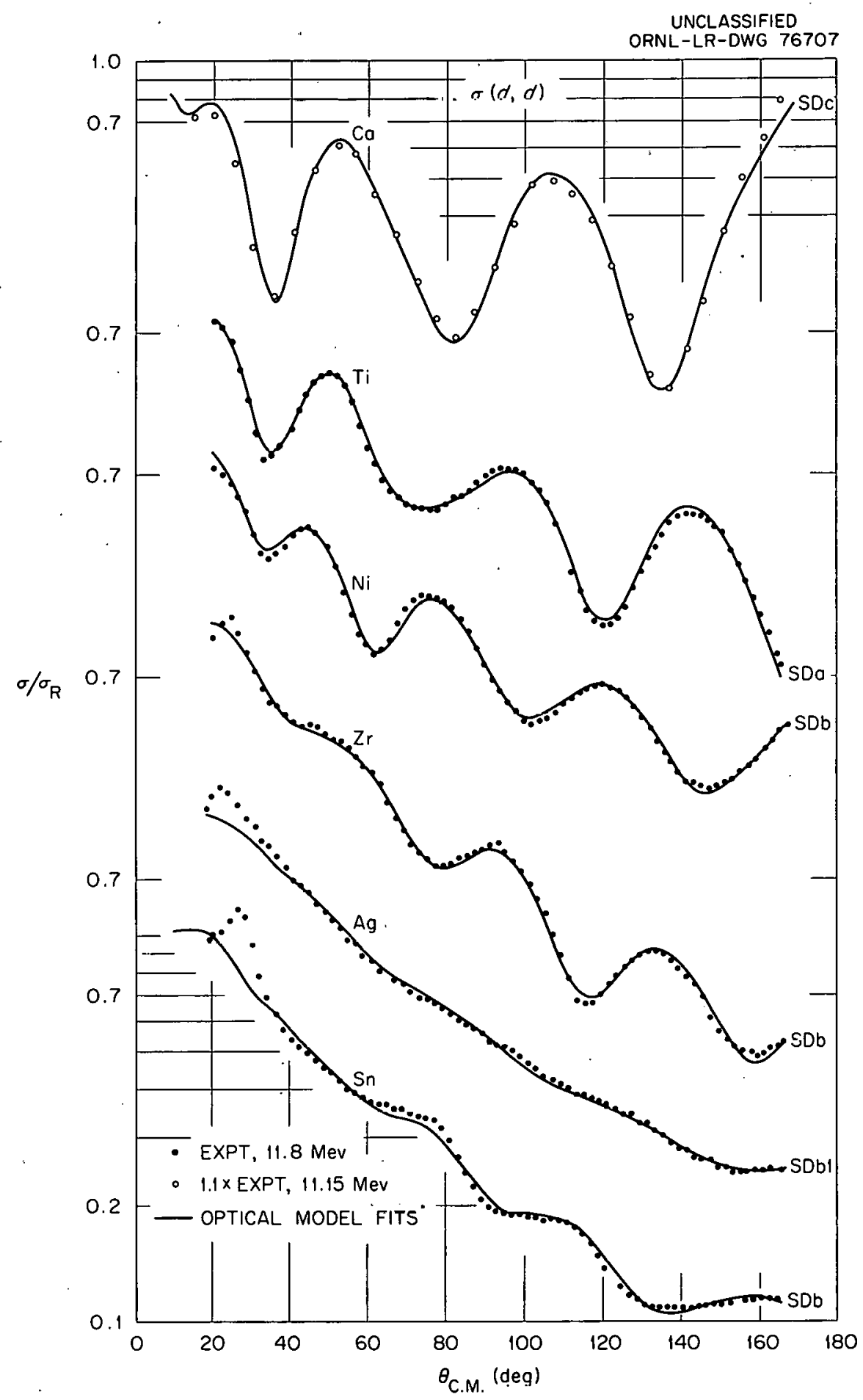

Fig. 3. 1. Good Fits Obtained for Six Different Experimental Cross Sections. 
to a relative minimum for $\chi^{2}$, not necessarily an absolute minimum.

Nine fits to the data for $11.8 \mathrm{-Mev}$ deuterons on nickel, as yielded by nine different optical-model potentials, are displayed in Figs. 3.2 and 3.3. The SD fits shown in Fig. 3.2 correspond to two separate $\chi^{2}$ minima in the six-dimensional parameter space describing SD potentials. Similarly, the five SP fits shown in Figs. 3.2 and 3.3 correspond to five separate $\chi^{2}$ minima in the sixdimensional SP-parameter space. Potentials intermediate in shape between two such distinct optimum potentials generally yield extremely poor fits (e.g., out of phase with the oscillations of $\left.\sigma_{\text {expt }} / \sigma_{R}\right)$

It is apparent from Figs. 3.2 and 3.3 that good fits can be obtained with any of several alternate potential forms. 'The parameters for the nine fits shown on nickel are listed in Table 3.1; they demonstrate that, even for a specified potential form, good or fair solutions can be found in many different regions of optical-model parameter.space.

For most of the 16 experimental cross sections studied, there exist good solutions ${ }^{6}$ related to all four of the nickel solutions shown in Fig. 3.2. The SDa-type fits for all targets are displayed in Figs. 3.4 and 3.5.

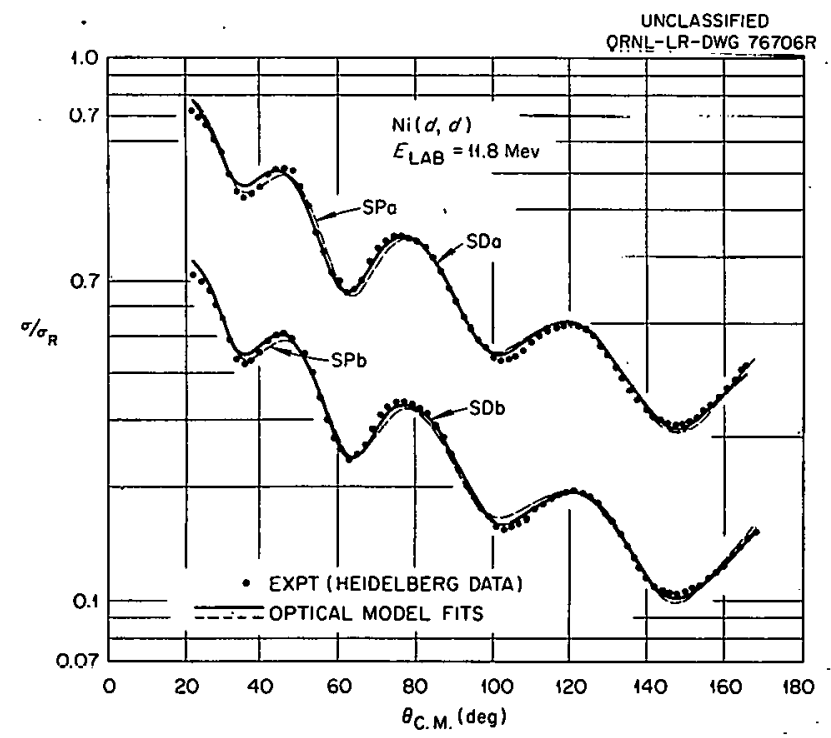

Fig. 3.2. Optical-Model Fits Corresponding to Four Different Scattering Potentials. The parameters for these potentials are given in Table 3.1.

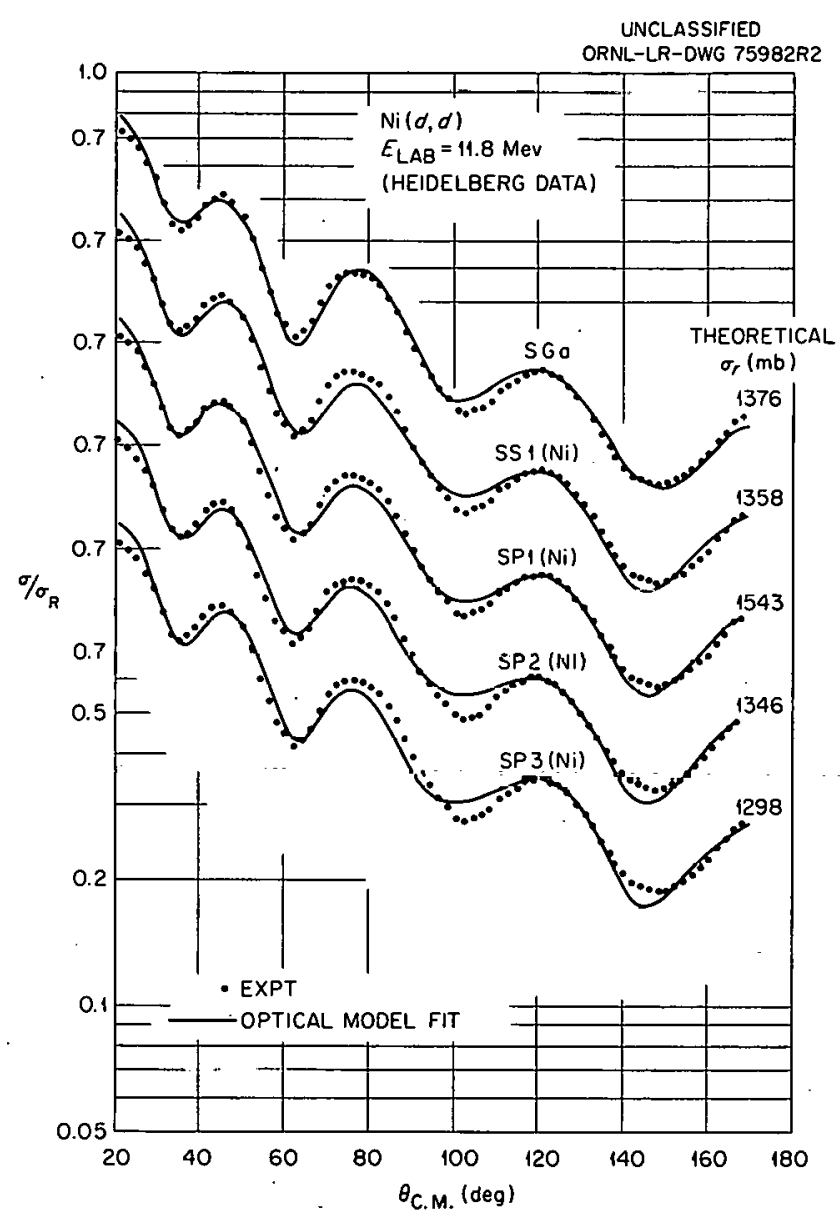

Fig. 3.3. Optical-Model Fits Corresponding to Five Difforant Srnttering Pntentials. The parameters for these potentials are given in Table 3. 1 .

Table 3.1 lists parameters for most of the fits shown in Figs. 3.1 to 3.5. Parameters for several other solutions of interest are also included. The quantity $\xi^{2}$, used in Table 3.1 as a numerical index to goodness of fit, is closely related to $x^{2}$ :

$$
\xi^{2}=\frac{1}{N} \sum_{\theta} \frac{\left[\sigma_{\text {theory }}(\theta)-\sigma_{\text {expt }}(\theta)\right]^{2}}{\left[0.05 \sigma_{\text {expt }}(\theta)\right]^{2}}
$$

\footnotetext{
${ }^{6} \mathrm{~A}$ complementary study of these and other data has been made by $C$. Perey and F. Perey, Neutron Physics Division; they used potentials similar to our SDa and SDb types. Their work emphasizes the variation of $V$ and $W$ with target and bombarding energy, under the condition that the four geometrical parameters have fixed values, the same for all targets and energies.
} 
Table 3.1. Samples of Potentials Yielding Optimum Fits $\left(\chi^{2}\right.$ Minima $)$

\begin{tabular}{|c|c|c|c|c|c|c|c|c|c|c|c|c|}
\hline $\begin{array}{c}E_{d} \\
(\mathrm{Mev})\end{array}$ & Target & Label & $\begin{array}{c}v \\
\text { (Mev) }\end{array}$ & $\begin{array}{c}r_{0} \\
\text { (fermis) }\end{array}$ & $\begin{array}{c}a \\
\text { (fermis) }\end{array}$ & $\begin{array}{c}W \\
(\mathrm{Mev})\end{array}$ & $\stackrel{{ }^{r} W}{\text { (fermis) }}$ & $\begin{array}{c}a_{W} \\
\text { (fermis) }\end{array}$ & $\begin{array}{c}\sigma_{r} \\
(\mathrm{mb})\end{array}$ & $N$ & $\xi^{2}$ & $\hat{\xi}^{2}$ \\
\hline 11.15 & \multirow{3}{*}{$\begin{array}{l}\mathrm{Ca} \\
\mathrm{Ca}(1.1 \sigma)^{a} \\
\mathrm{Ca}(1.1 \sigma)^{a}\end{array}$} & $\mathrm{SDa}$ & 67.5 & 0.974 & 1.054 & 58.3 & 1.516 & 0.489 & 1214 & 31 & 6.7 & 10.4 \\
\hline 11.15 & & $\mathrm{SDa}$ & 67.6 & 0.978 & 1.022 & 56.4 & 1.518 & 0.490 & 1191 & 31 & 5.1 & \multirow[t]{2}{*}{8.8} \\
\hline 11.15 & & $\mathrm{SDc}$ & 32.7 & 0.880 & 1.233 & 25.1 & 1.731 & 0.639 & 1452 & 31 & 0.82 & \\
\hline 11.8 & $\mathrm{Ti}$ & $\mathrm{SDa}$ & 49.8 & 1.183 & 0.817 & 48.2 & 1.434 & 0.671 & 1384 & 74 & 0.51 & 0.60 \\
\hline 11.8 & \multirow{2}{*}{$\begin{array}{l}\mathrm{Fe} \\
\mathrm{Fe}^{(0.9 \sigma)^{a}}\end{array}$} & $\mathrm{SDa}$ & 58.4 & 1.103 & 0.836 & 53.3 & 1.353 & 0.733 & 1392 & 73 & 0.73 & 0.75 \\
\hline 11.8 & & $\mathrm{SDa}$ & 57.3 & 1.105 & 0.897 & 55.0 & 1.375 & 0.713 & 1427 & 74 & 0.42 & 0.44 \\
\hline \multirow[t]{9}{*}{11.8} & \multirow[t]{9}{*}{$\mathrm{Ni}$} & $\mathrm{SDa}$ & 56.4 & 1.164 & 0.865 & 56.6 & 1.427 & 0.665 & 1395 & 74 & 0.43 & \multirow[t]{9}{*}{0.45} \\
\hline & & SDb & 79.5 & 1.274 & 0.739 & 81.6 & 1.389 & 0.624 & 1396 & 74 & 0.31 & \\
\hline & & $\mathrm{SPa}$ & 60.5 & 1.121 & 0.860 & 7.59 & 1.834 & 0.537 & 1357 & 74 & 0.50 & \\
\hline & & $\mathrm{SPb}$ & 85.1 & 1.233 & 0.735 & 11.25 & 1.717 & 0.567 & 1354 & 74 & 0.64 & \\
\hline & & $\mathrm{SGa}_{2}$ & 55.9 & 1.171 & 0.872 & 11.51 & 1.347 & 1.881 & 1357 & 74 & 0.54 & \\
\hline & & SS1 & 59.1 & 1.544 & 0.535 & 14.7 & $r_{0}$ & $a$ & 1358 & 73. & 1.2 & \\
\hline & & $\mathrm{SP} 1$ & 68.0 & 1.459 & 0.431 & 16.6 & 1.291 & 1.134 & 1543 & 73 & 1.5 & \\
\hline & & SP2 & 91.8 & 1.520 & 0.502 & 32.2 & 1.064 & 0.289 & 1346 & 73 & 1.4 & \\
\hline & & SP3 & 35.5 & 1.570 & 0.524 & 19.0 & 1.244 & 0.368 & 1298 & 73 & 1.6 & \\
\hline 11.15 & $\mathrm{Cu}$ & $\mathrm{SDa}$ & 64.7 & 1.065 & 0.987 & 57.8 & 1.457 & 0.711 & 1517 & 31 & 0.67 & 0.71 \\
\hline 11.8 & $\mathrm{Cu}$ & $\mathrm{SDa}$ & 60.6 & 1.133 & 0.868 & 52.7 & 1.414 & 0.716 & 1476 & 74 & 0.47 & 0.47 \\
\hline 11.8 & $\mathrm{Zn}$ & $\mathrm{SDa}$ & 65.9 & 1.095 & 0.824 & 55.9 & 1.348 & 0.774 & 1460 & 74 & 0.53 & 0.55 \\
\hline 11.8 & $\mathrm{Zr}$ & SDa & 63.0 & 1.1914 & 0.725 & 37.4 & 1.307 & 0.859 & 1383 & 73 & 0.32 & 0.36 \\
\hline 11.8 & & $\mathrm{SDa}$ & 69.6 & 1.150 & 0.697 & 41.0 & 1.262 & 0.810 & 1214 & 72 & 0.35 & 0.35 \\
\hline 11.8 & $\mathrm{Nb}^{(0.9 \sigma)^{\sigma}}$ & $\mathrm{SDa}$ & 79.9 & 1.009 & 0.896 & 38.8 & 1.412 & 0.771 & 1399 & 74 & 0.14 & 0.33 \\
\hline \multirow[t]{4}{*}{11.88} & \multirow[t]{4}{*}{$\mathrm{Ag}^{b}$} & SDaa & 56.9 & 0.967 & 0.938 & 52.9 & 1.276 & 0.826 & 1248 & 68 & 0.29 & \\
\hline & & $\mathrm{SDa}$ & 90.5 & 0.940 & 0.889 & 65.5 & 1.229 & 0.830 & 1243 & 68 & 0.29 & \multirow[t]{3}{*}{0.30} \\
\hline & & SDbl & 149.5 & 0.866 & 0.874 & 73.9 & 1.204 & 0.836 & 1250 & 68 & 0.29 & \\
\hline & & $\mathrm{SDb} 2$ & 114.2 & 1.039 & 0.791 & 85.2 & 1.186 & 0.817 & 1240 & 68 & 0.29 & \\
\hline \multirow[t]{4}{*}{11.8} & \multirow[t]{4}{*}{$\mathrm{Sn}$} & SDaa & 78.3 & 0.786 & 1.046 & 29.9 & 1.731 & 0.866 & 2051 & 73 & 1.91 & \\
\hline & & $\mathrm{SDa}$ & $125.7^{\circ}$ & 0.740 & 1.004 & 30.9 & 1.729 & 0.874 & 2074 & 73 & 1.93 & \\
\hline & & $S D b$ & 196.9 & 0.709 & 0.906 & 30.1 & 1.736 & 0.878 & 2079 & 73 & 1.93 & \\
\hline & & SDbb & 299.5 & 0.644 & 0.922 & 30.8 & 1.728 & 0.883 & 2083 & 73 & 1.95 & \\
\hline \multirow[t]{3}{*}{11.8} & \multirow[t]{3}{*}{$\mathrm{Sn}^{c}$} & SDaa & 71.5 & 0.821 & 1.027 & 30.3 & 1.807 & 0.858 & 2218 & 65 & 0.47 & \\
\hline & & $\mathrm{SDa}$ & 117.3 & 0.782 & 0.954 & 30.7 & 1.806 & 0.865 & 2229 & 65 & 0.46 & \\
\hline & & $\mathrm{SDb}$ & 191.7 & 0.702 & 0.971 & 32.0 & 1.792 & 0.872 & 2229 & 65 & 0.47 & \\
\hline
\end{tabular}

${ }^{a}$ These results refer to the analysis of renormalized data.

${ }^{b}$ Experimental points below $31^{\circ}$ were ignored for all searches on $\mathrm{Ag}$ data.

${ }^{c}$ Experimental points below $33^{\circ}$ were ignored for these searches on $\mathrm{Sn}$ data. 


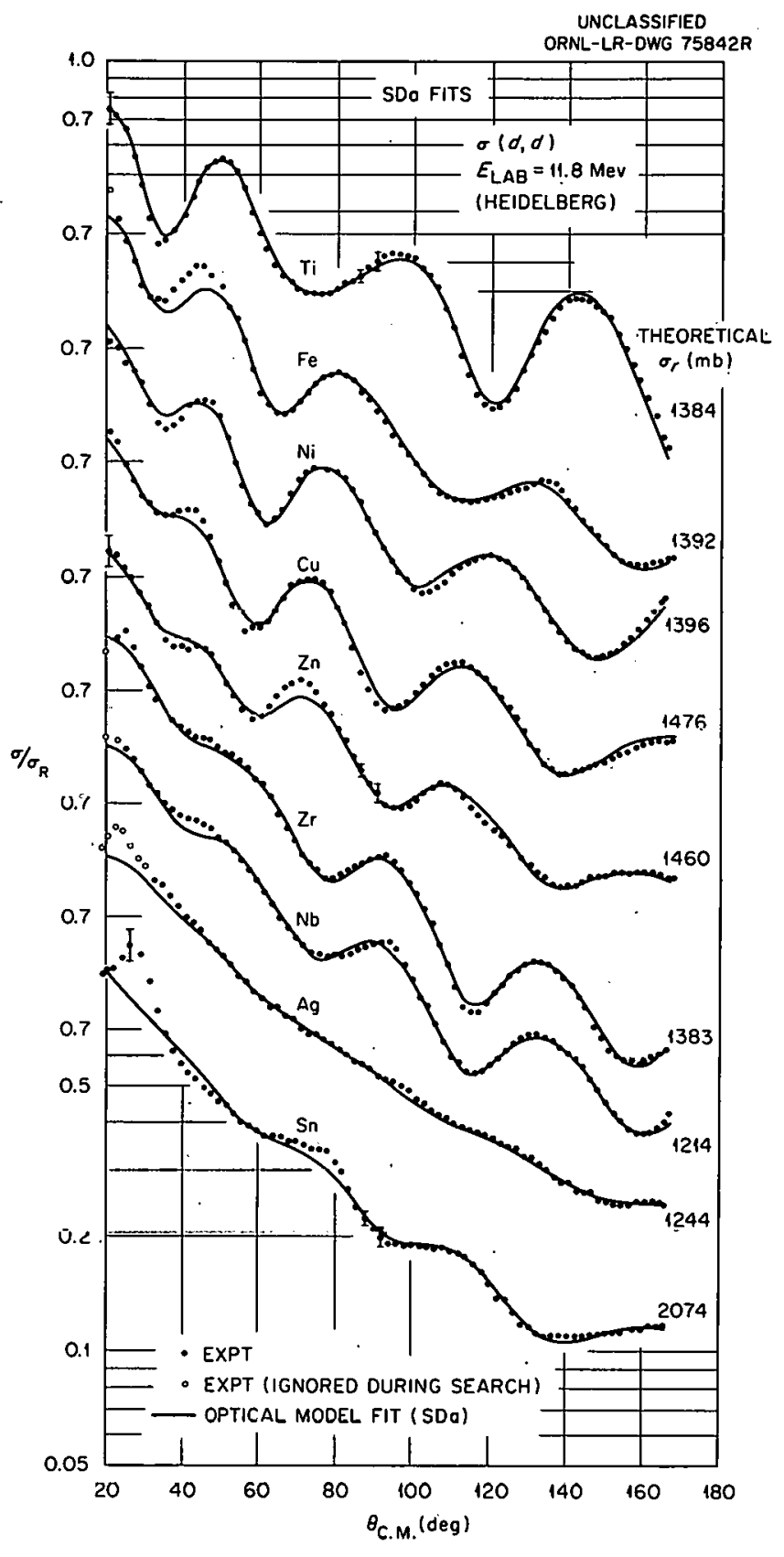

Fig. 3.4. Optimized SDa-Type Fits to 11.8-Mev Data. (See ref 5.)

Here $N$ is the number of experimental points used during search. The index $\hat{\xi}^{2}$ gives the changed value of $\xi^{2}$ when $r_{0}$ is fixed at 1.133 fermis, while all other parameters remain adjustable.
Different converged solutions, $\sigma_{\text {theory }}(\theta)$, corresponding to separate $\chi^{2}$ minima for the same measured cross section, often resemble each other even more closely than they reproduce $\sigma_{\text {expt }}(\theta)$. Compare, for example, the SDb fits shown in Fig. 3.1 with the SDa fits in Fig. 3.3. The similarity also extends to the theoretical reaction cross sections $\sigma_{r}$ (see Table 6.1) and to the computed complex phase shifts.

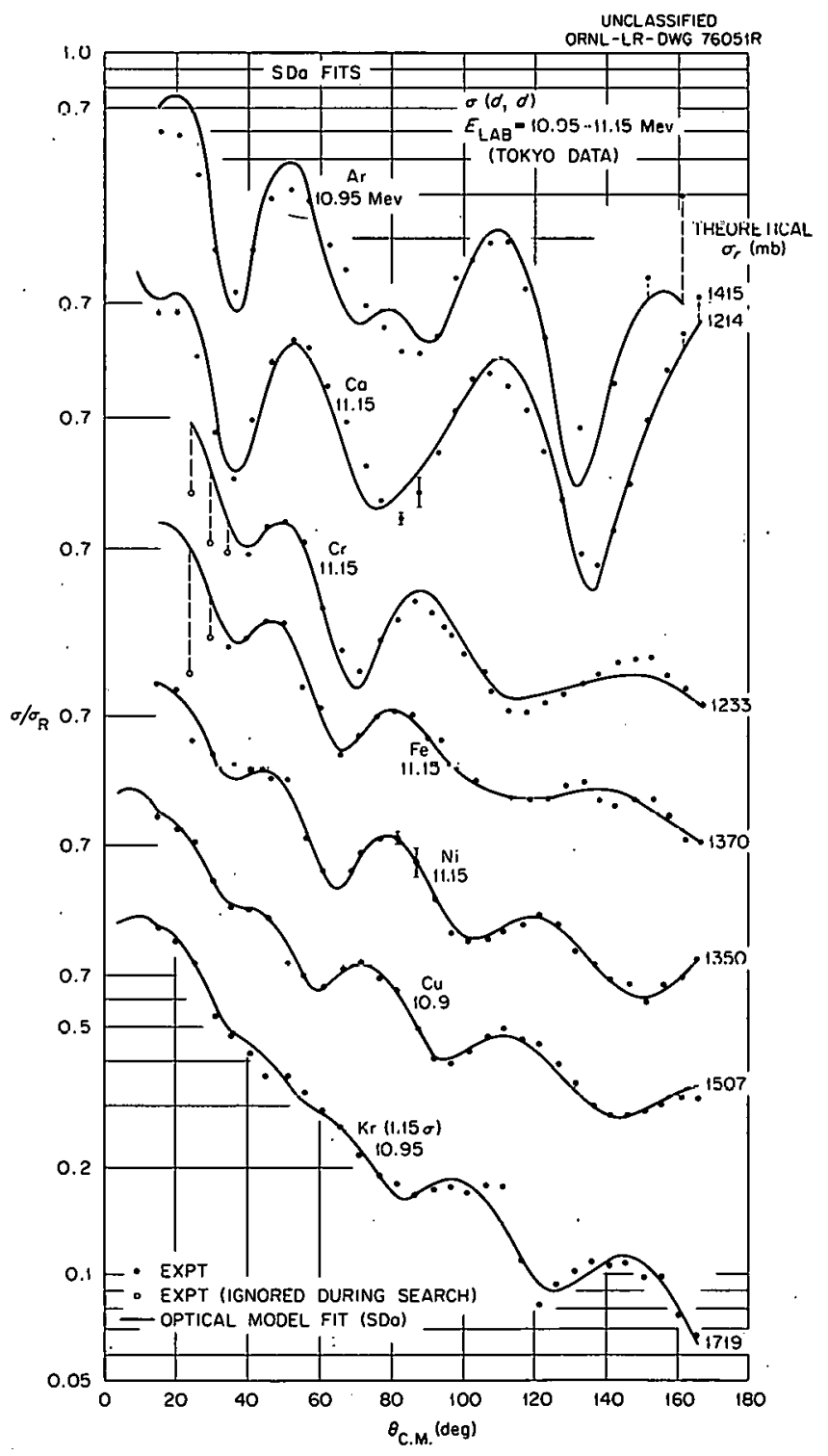

Fig. 3.5. Optimized SDa-Type Fits to $11-$ Mev Data. (See ref 5.) 


\section{$\mathrm{He}^{3}$ ELASTIC SCATTERING}

Data for elastic scattering of $\mathrm{He}^{3}$ from nuclei have been accumulating for the past few years 7,8 and have been analyzed in terms of the optical model. ${ }^{8,9}$ One remarkable feature of these analyses is the large depths of the imaginary wells used to fit the data, the depths being comparable to or greater than the depth of the real potential. This led to the suggestion that $\mathrm{He}^{3}$ particles are even more strongly absorbed than alpha particles, supposedly because the $\mathrm{He}^{3}$ particle is more easily dissociated by the nuclear medium. Such an interpretation is questionable, because a large imaginary potential gives risc to effects similar to those given by the real potential and also causes absorption. In addition, the optical-model calculations 8,9 are attempts to provide "best fits" to the measured cross sections, and they show large fluctuations of the parameters as a function of atomic mass.

It is of interest to see if the main features of the measured cross sections can be reproduced by an optical potential whose parameters vary slowly with atomic mass. This property has been demonstrated for nucleons, ${ }^{10}$ deuterons, ${ }^{11}$ and alpha particles. ${ }^{12}$

For these reasons the available $\mathrm{He}^{3}$ data is being reanalyzed. Calculations for the elastic scattering of $29-\mathrm{Mev} \mathrm{He}^{3}$ ions from vanadium, nickel, and copper are shown in Fig. 3.6. It is found that these three cases can be satisfactorily fitted with the same optical potential; the essen-

${ }^{7}$ D. J. Bredin et al., Proc. Roy. Soc. 258, 202 (1960); G. W. Greenlees and P. C. Rowe, Nucl. Phys. 15, 687 $(1960) ;$ H. E. Wegner and W. S. Hall, Bull. Am. Pbys. Soc. 3, 338 (1958); private communication, 1960; H. E. Wegner and W. S. Hall, Pbys. Rev. 119, 1654 (1960); S. Hinds and R. Middleton, Proc. Phys. Soc. 75, 745 (1960); J. R. Priest et al., Pbys. Rev. 119, 1295 (1960); G. Parry et al., Proc. Phys. Soc. 77, 230 (1961); D. A. Bromley and E. Almquist, Rept. Progr. Phys. 23, 544 (1960).

${ }^{8}$ J. Aguilár et al., Proc. Roy. Soc. 257, 13 (1960); J. Aguilar et al., Nucl. Phys. 25, 259 (1961); G. W. Greenlees et al.. Nucl. Pbys. 24, 334 (1961); H. M. Sen Gupta et al., Nucl. Phys. 38, 361 (1962).

${ }^{9} \mathrm{P}$. E. Hodgson, Nucl. Pbys. 21, 28 (1960); 23, 499 (1961); Proc. Pbys. Soc. 77, 997 (1961).

${ }^{10} \mathrm{~F}$. Bjorklund and S. Fernbach, Pbys. Rev. 109. 1295 (1958); F. Perey, to be published.

${ }^{11}$ E. C. Halbert, to be published; C. Perey and F. Perey, to bc publishcd.

${ }^{12}$ G. Igo and R. Thaler, Phys. Rev. 106, 1126 (1957). tial differences are accounted for by the mass and charge differences. The parameters of this well are of the "usual" type, similar to the parameters found in optical-model analyses of the scattering of other particles. If we take the mean free path of the particle in the potential as a measure of absorption, the ratio found for copper from this prediction relative to the previous analysis is 0.3 , indicating less absorption. Similar remarks hold for nickel and vanadium. When the reaction cross sections are compared, it is seen that the ORNL calculations show a smooth dependence with mass and are smaller than those previously reported.

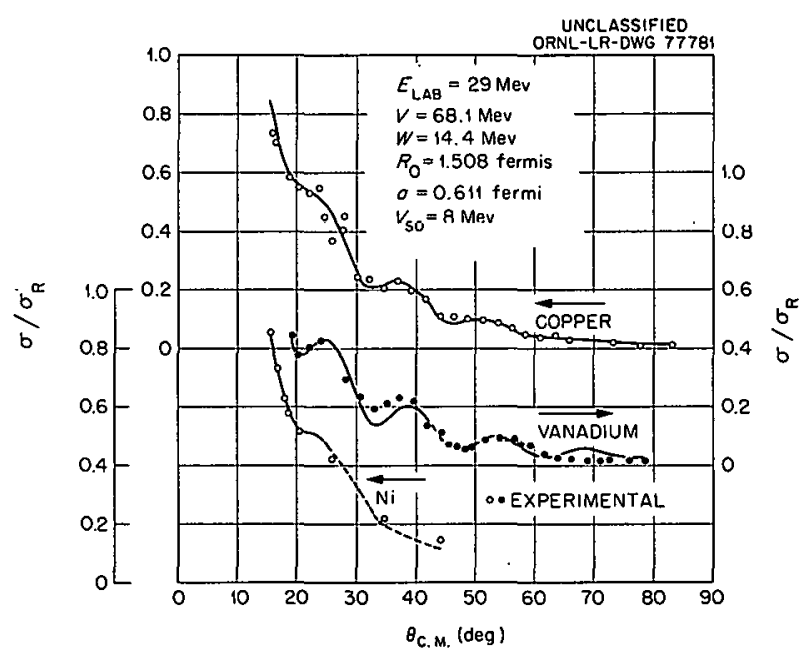

Fig. 3.6. Optical-Model Calculations for the Scattering of $\mathrm{He}^{3}$ lons from Vanadium, Nickel, and Copper, Compared with Experiment.

In the following tabulation, reaction cross sections for $\mathrm{He}^{3}$ scattering from vanadium, nickel, and copper, as computed with the potential shown in Fig. 3.6 and from the calculations of Hodgson ct al., 13 arc compared:

\begin{tabular}{lcc}
\multirow{2}{*}{ Target } & \multicolumn{2}{c}{$\sigma R(\mathrm{mb})$} \\
\cline { 2 - 3 } & ORNL & Hodgson et al. \\
Vanadium & 1381 & 1521 \\
Nickel & 1396 & 1426 \\
Copper & 1439 & 1739
\end{tabular}

${ }^{13}$ G. W. Greenlees et al., Nucl. Pbys. 24, 334 (1961); P. E. Hodgson, Nucl. Phys. 21, 28 (1960). 


\section{ELASTIC AND INELASTIC SCATTERING OF CARBON IONS FROM CARBON}

A series of measurements have been made on the Yale Linac to investigate the scattering of 127Mev carbon ions from carbon. ${ }^{14,15}$ The following effects were observed: angular distributions for elastic scattering, single excitation of one of the carbon nuclei to the $4.43-\mathrm{Mev}\left(2^{+}\right)$state, and excitation of the $14-\mathrm{Mev}\left(4^{+}\right)$state.

These data were analyzed in terms of the collective model by assuming that the two-body aspects of the problem are more important than the many-body effects. Calculations have proceeded in two ways: (1) With the reactions treated as perturbations, the elastic scattering was calculated by assuming a spherical well tor the optical potential; the results are shown in Fig. 3.7. In this approximation the first term in the aspherical part of the interaction leads to excitation of the first excited state of one of the nuclei. ${ }^{16}$ (2) $A$ more exact treatment of the problem leads to a set of coupled equations, ${ }^{17}$ which couple the elastic channel to the $2^{+}$and $4^{+}$excitations of one of the nuclei; the rotational model is assumed.

The results of these calculations for single excitation of the $2^{+}$first excited state are shown, along with the measurements, in Fig. 3.8. Two curves are calculations in distorted-wave approximation, with and without Coulomb excitation. The third is the prediction of the strong coupling code, with the same parameters used in the distortedwave calculation except that the radius parameter is reduced.

The value found for the deformation parameter $\beta$ is somewhat larger than the value for $\beta$ found from similar analyses of alpha-particle inelastic scattering or proton inelastic scattering but is within the uncertainty of the Coulomb excitation measurement.

The predictions of the strong coupling code for single excitation of the $4^{+}$state in carbon are

\footnotetext{
${ }^{14} \mathrm{~K}$. H. Wang, S. D. Baker, and J. A. McIntyre, Phys. Rev. 127, 187 (1962).

${ }^{15}$ G. Garvey; A. M. Smith, and J. C. Hiebert, to be published.

${ }^{16} \mathrm{E}$. Rost and N. Austern, Pbys. Rev. 120, 1375 (1960); R. H. Bassel et al., Phys. Rev. 128, 2693 (1962).

${ }^{17}$ B. Buck, Phys. Rev. 127, 940 (1962).
}

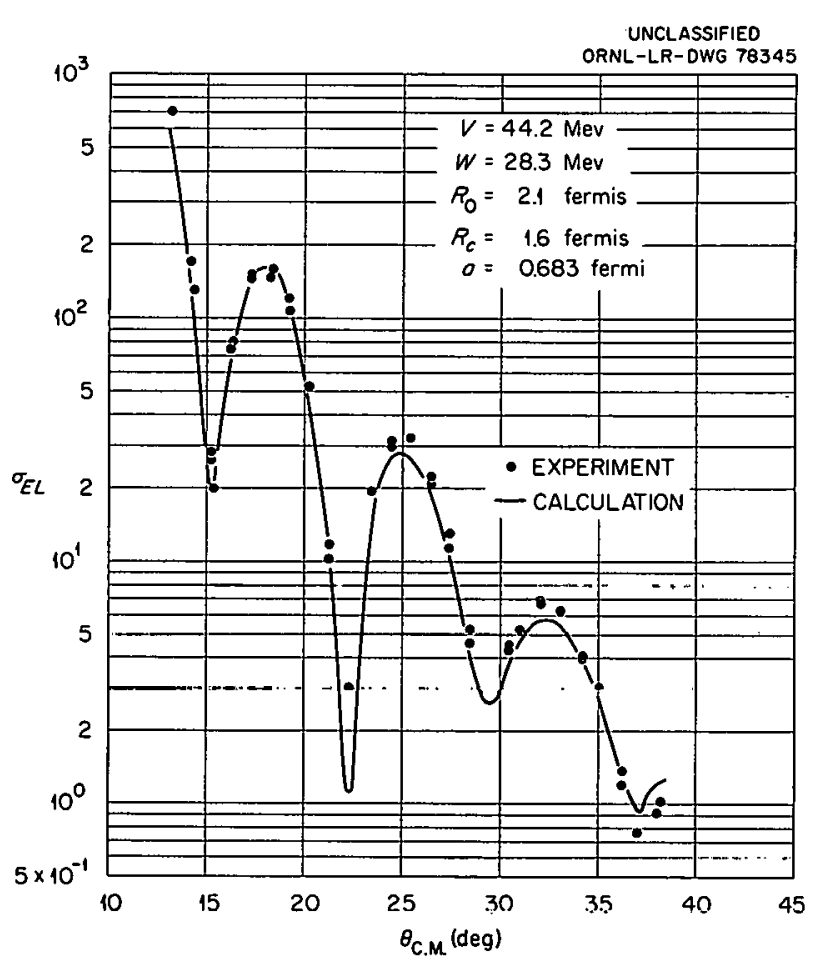

Fig. 37. Optical-Model Prediction for the Elastic Scattering of 127-Mev Carbon Ions from Carbon.

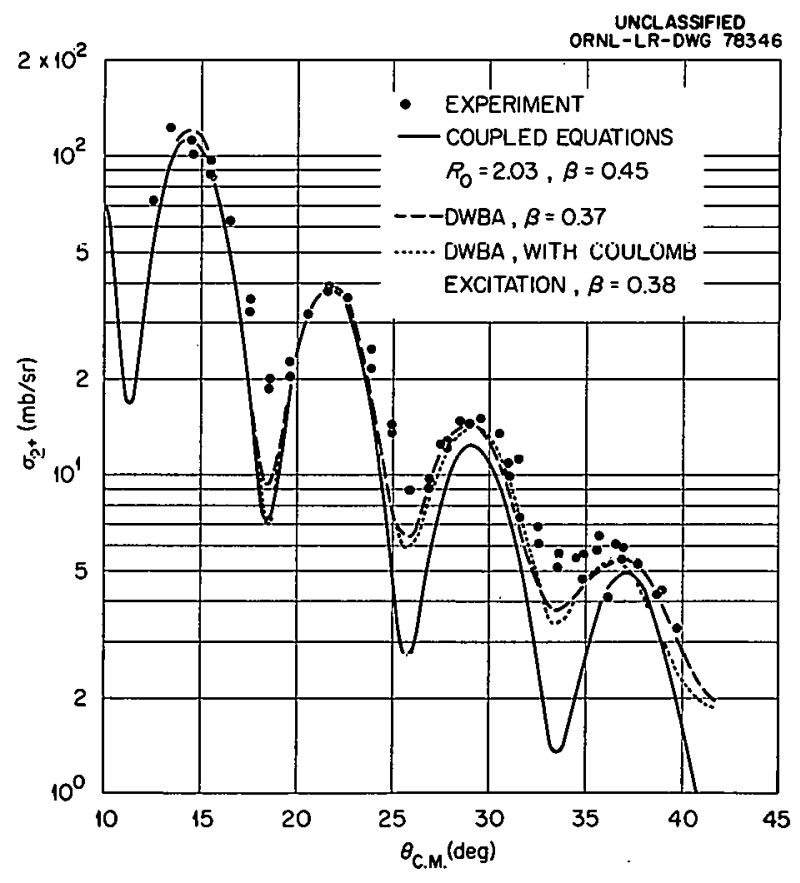

Fig. 3.8. Predicted and Experimental Cross Sections for the "Single" Excitation of the 4.43-Mev $\left(2^{+}\right)$State in Carbon by Carbon. 
compared with experimental measurements in Fig 3.9. Although details of the angular distribution are not well reproduced, the magnitude is in good agreement with experiment.

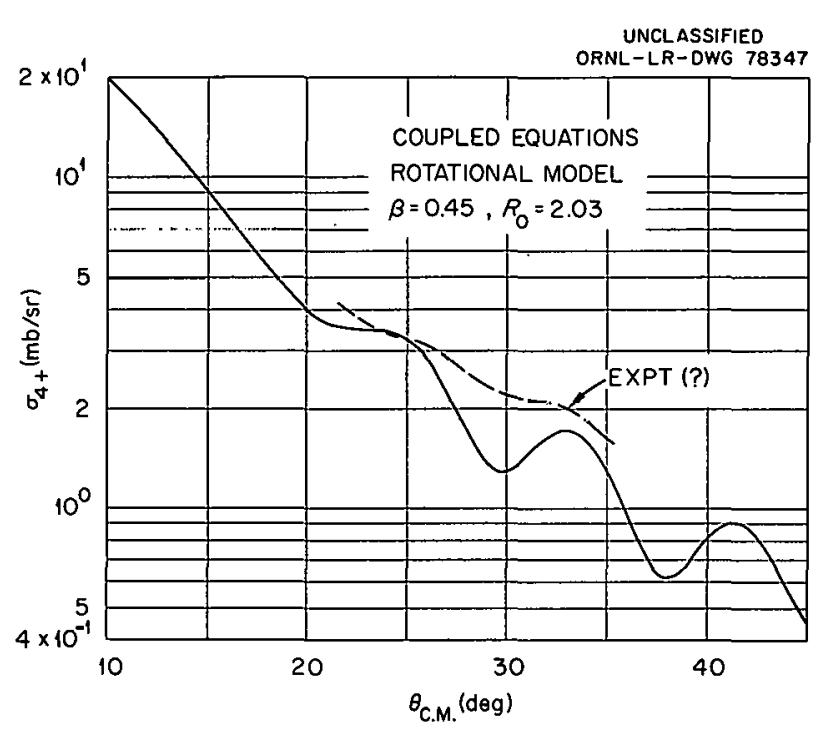

Fig. 3.9. Predicted and Experimental Cross Sectlons for the "Single" Excitation of the 14-Mev (4h) State in Carbon by Carbon.

\section{FINITE-RANGE CORRECTION TO DISTORTED. WAVE APPROXIMATION}

The distorted-wave approximation (DWA) in zerorange approximation for stripping has shown discrepancies with experiment. The use of opticalmodel wave functions which explain the elastic scattering of deuterons and protons does not improve the situation but, instead, increases the confusion. As shown in the section on elastic scattering of deuterons, there are many potentials which give the same elastic scattering (i.e., asymptotic wave function) but give different predictions for stripping. It was discovered that many of these ambiguities arise from a potential change which allows an additional half wavelength (or multiple thereof) for the lower partial waves, thus changing the character of the wave function in the interior. This has also been shown independently by F. Perey, Neutron Physics Division.
It has been suggested that the contribution from the interior is overestimated by the zero-range approximation, which takes the product of the neutron-proton force and the deuteron internalwave function as a delta function. A more realistic calculation would take into account the finite range of the neutron-proton force and the extension of the deuteron. Such a modification implies going from a threefold to a sixfold integral.

A DWA code was written which replaces the zero-range approximation,

by

$$
V\left(r_{n p}\right) \phi\left(r_{n p}\right)=A \delta\left(r_{n p}\right)
$$

$$
V\left(r_{n p}\right) \phi\left(r_{n p}\right)=D\left(r_{n p}\right)
$$

where $D\left(r_{n p}\right)$ can be taken as a Gaussian,

$$
D\left(r_{n p}\right)=A N_{G} e^{-\left(r_{n p}\right)^{2}} /\left(R_{G}\right)^{2}
$$

or as the Yukawa form,

$$
D\left(r_{n p}\right)=A N_{Y} \frac{e^{-\left|r_{n p}\right| / R_{Y}}}{\left|r_{n p}\right| / R_{Y}}
$$

For flexibility the function which describes the motion of the bound particle relative to the target nucleus is not restricted to a Gaussian but can be taken in any desired form. To conserve computer time and still retain accuracy, the code performs either linear or quadratic interpolation of this function.

Spin-orbit interactions can be included in initial and/or final channels. At present the code is restricted to $l_{n} \leqq 4$ and to 30 partial waves in entrance and exit channels. A comparison of the code with explicit analytic expressions shows agreement within $1.3 \%$ over a range of four decades in the angular distribution. Typical IBM 7090 running time is from 4 to $8 \mathrm{~min}$, in comparison with 1 to $2 \mathrm{~min}$ for the zero range.

Although the Yukawa form is preferred on theoretical grounds, it was found that if the range $R_{\mathrm{Y}}$ is chosen so that the zero-momentum components agree with the zero-momentum components of the Gaussian function of range $R_{\mathrm{G}}$, there is essentially no difference between the predicted cross sections. Since, the Gaussian function requires substantially less computing time, subsequent studies have been restricted to this form. 
A theoretical calculation for $11-\mathrm{Mev}$ deuterons on calcium going to a fictitious state in $\mathrm{Ca}^{41}$ with $Q=0$ is shown in Fig. 3.10. Four curves are shown: the upper set take radial matrix elements from the origin and are labeled ZR (zero range) and $F R$ (finite range); the lower set correspond to taking radial integrals from 4.1 fermis. It is seen that for a small $Q$ the finite-range effects are negligible. In a similar calculation for $Q=10 \mathrm{Mev}$ (Fig. 3.11'), the finite-range effects are more noticeable but mostly amount to a renormalization of the calculated cross section. The shape of the angular distribution is little affected; however, the finite-range prediction disagrees with.

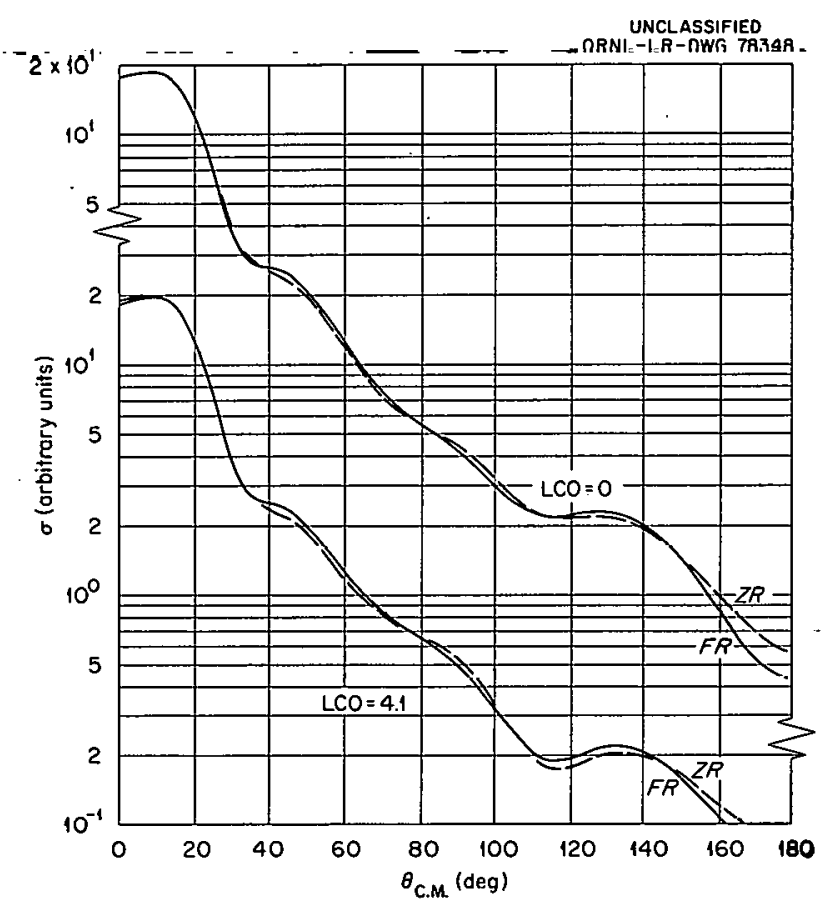

Fig. 3.10. Comparison of Zero- and Finite-Range Calculation for $\mathrm{Ca}^{40}\left(d_{0} p\right) \mathrm{Ca}^{41} ; l=1$ with $Q=0$. The Goussian range is taken as 1.5 fermis. the zero-range prediction, even with a lower radial cutoff of 4.1 fermis. These calculations and others directly related to experiment tentatively suggest that the finite-range effects, with a reasonable range, do not markedly improve the situation over the zero range. Other effects, such as polarization of the deuteron, nonlocality, and strong coupling, may be important and are being studied.

Several extensions of the code are planned: a study of the heavy-ion nucleon-transfer experiments, where the range of the force is quite large; and a study of the $(n, p)$ reaction, where the use of a zero-range force is wrong in principle.

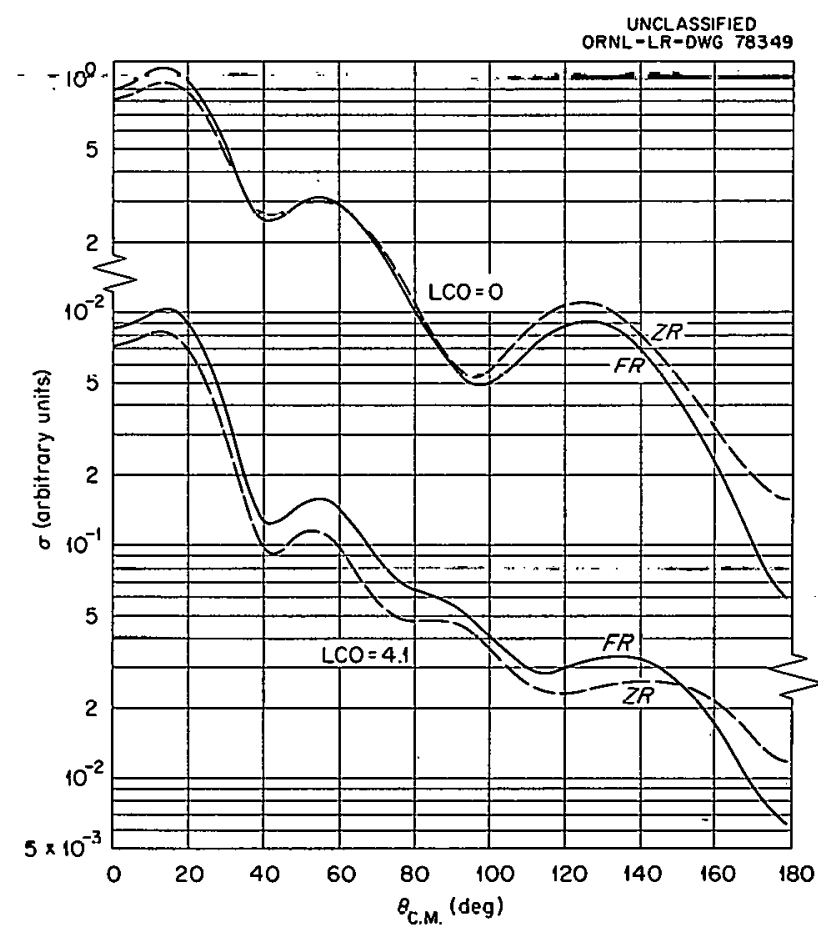

Fig. 3.11. Comparison of Zero- and Finite-Range Calculation for $\mathrm{Ca}^{40}(d, p) \mathrm{C}^{41} ; l=1$ with $Q=10 \mathrm{Mev}$. The Gaussian range is taken as 1.5 fermis. 


\section{Cyclotron Operations}

\section{OPERATION OF THE OAK RIDGE ISOCHRONOUS CYCLOTRON}

During the first three months of 1962, efforts were primarily devoted to installing and testing the various components of ORIC. For example, the source and probe mechanism with its vacuum lock, carriage rails, and associated electrical controls was installed, and successful operational checkouts were made. Similarly, the water and vacuum systems with their various interlocks and limit switches were installed and tested.

In the early part of March the operations group started 24-hr coverage five days a week. This coincided with the start of initial test operation of the machine, which included getting operable pressures, providing adequate cooling water to the various components, stabilizing rf excitation, and accelerating a proton beam. The best vacuum obtained about this time was $\sim 1.9 \times 10^{-6}$ torr, with a rate of rise of $\sim 0.00045 \mu / \mathrm{sec}$. With the tank under vacuum the temporary if system was successfully operated at $7.95 \mathrm{Mc} / \mathrm{sec}$ with up to $18 \mathrm{kvp}$ on the dee. Details of the testing program are described in Sec 6 .

In mid-March the first internal proton beam was obtained at the full radius of 31.5 in. The proton energy was established as $8.0 \pm 0.1 \mathrm{Mev}$ through bombardment of stacked copper foils and subsequent counting of the activity and calculations based on range-energy curve and foil thickness. The testing program continued for about three months, during which time numerous internal beam bombardments were made to verify the operational characteristics of ORIC.

Some deflection experiments were made during this testing period. The initial deflection of the internal circulating beam was achieved in the first few weeks of June. A more detailed discussion of this appears in $\operatorname{Sec} 6$.
On July 9, 1962, the testing program on ORIC was terminated to begin installation of the permanent if system and other associated equipment (electrostatic channel, magnetic channel, and the ion optics) and their supplies, utilities, and controls.

Operational checkout and testing of the permanent if system was begun in October 1962. This phase of activities continued until, on December 20, 1962, a dee-to-ground potential of $\sim 50 \mathrm{kv}$ was obtained. With this initial success, high-energy beams are expected to be available soon in the ORIC.

\section{OPERATION OF THE ORNL 63-INCH CYCLOTRON}

As reported earlier, scheduled operation of the 63-Inch Cyclotron was discontinued October 31, 1961. Since then the machine has been kept in standby condition, to be operated occasionally as needed. During the past year, about 30 runs of from $2 \mathrm{~min}$ to several hours duration were made for the Heavy-Particle Physics Group. Although these runs were made at widely scattered intervals, the machine operation was reasonably stable and trouble free.

\section{OPERATION OF THE ORNL 86-INCH CYCLOTRON}

The responsibility for operation of the 86-Inch Cyclotron was assumed by the Isotopes Division on December 1, 1961. Since then the Proton Physics Group has continued to use the machine for about 36 scheduled hours per wcek. The Isotopes Division is paid the current hourly rate for use of the machine. 


\section{Accelerator Development}

\author{
E. E. Gross \\ E. D. Hudson \\ R. J. Jones \\ R. S. Livingston \\ R. S. Lord \\ J. E. Mann
}
M. B. Marshall
J. A. Martin
C. E. Murphy ${ }^{1}$
T. E. Northup ${ }^{1}$
E. G. Richardson
F. M. Kusseil ${ }^{2}$

\author{
W. R. Smith \\ W. H. White, Jr. ${ }^{3}$ \\ R. E. Worsham \\ N. F. Ziegler \\ A. Zucker
}

A number of major advances in accelerator development and technology were made during the year. Experimental studies on the eight-sector electron model, Analogue II, led to very successful deflection of the beam in November 1962. Although the investigations are still incomplete, the results obtained have removed one of the major uncertainties associated with the proposed $\mathrm{Mc}^{2}$ cyclotron. This uncertainty centered around the radioactivity problem resulting from the residual undeflected beam being dumped onto the structure of the cyclotron. Estimates of the radiation levels that would be encountered had varied widely; they can now be narrowed to much more reliable calculations. These new estimates were incomplete at the close of the year and will be described in subsequent reports.

An innovation in cyclotron design, designated the Separated Orbit Cyclotron (SOC) and sometimes called the "beehive" or "helitron," was conceived, and a preliminary report was prepared. The significance of this new device is difficult to assess accurately at this early stage, but it is believed that with additional development the machine may emerge as an important new type of accelerator for the $\frac{1}{2}$ - to $10-\mathrm{Bev}$ energy range.

The Laboratory made a presentation of its plans and projected experimental program for the $\mathrm{Mc}^{2}$

\footnotetext{
${ }^{1}$ Engineering and Mechanical Division.

${ }^{2}$ On leave from Rutherford Laboratory, Harwell.

${ }^{3}$ Instrumentation and Controls Division.
}

cyclotron to the Ramsey Panel. ${ }^{4}$ It is experted that the report of the panel will become available during the spring or summer of 1963. These plans and other details of the project are also summarized in a status report ${ }^{5}$ issued in June 1962.

A special conference was held at Gatlinburg, Tenn., November 12-13, 1962, on the general subject of "Research in Physics Below One Bev." A summary report of this conference will appear in the March 1963 issue of Pbysics Today. The general consensus of the delegates was that a rich and important program of research awaits the high-intensity "meson factories."

\section{CYCLOTRON ANALOGUE II}

Successful operation of the eight-sector electron cyclotron was reported last year. This machine modcls the particle dynamics expected in the 810-Mev $\mathrm{Mc}^{2}$ proton cyclotron under study at ORNL. The Analogue is being used to verify the main features of the design and performance of that machine. In recent tests a beam-deflection efficiency of $85 \%$ was obtained. This high deflection efficiency will considerably ease the problem of keeping the activation of internal components of the large proton cyclotron within manageable limits.

\footnotetext{
${ }^{4}$ Special panel to the GAC-PSAC to recommend levels and directions for future high-energy accelerator projects.

${ }^{5}$ The $\mathrm{Mc}^{2}$ Isochronous Cyclotron, ORNL-3324 (June $15,1962)$.
} 
The Analogue uses an eight-sector moderatespiral magnetic-field configuration produced entirely by air-cored windings to produce the field distribution shown in Fig. 5.1. Several advantages accrued from the choice of an iron-free system, chief among which was the ability to produce a highly precise magnetic field with wellknown focusing properties without recourse to a lengthy field measurement and correction program.

The central magnetic field in the Analogue is approximately 41.9 gauss; the orbit frequency is $117 \mathrm{Mc} / \mathrm{sec}$. The cyclotron unit, $c / \omega$, is $16.00 \mathrm{in}$. The maximum energy which can be achieved (limited by loss of axial focusing) is approximately $530 \mathrm{kev}$. The energy of the extracted beam, at a mean radius of $13.4 \mathrm{in}$., is about 445 kev.

During the initial operation of the Analogue, a major fraction of the beam was lost at a radius of 10.4 in.; also, an axial oscillation of the beam envelope was noted at $12.6 \mathrm{in}$. The trouble at 10.4 in. was at first thought to be caused by either the $\nu_{r}=\frac{4}{3}$ or $8 / 6$ resonance, but it was later shown to be the difference-coupling re sonance $\nu_{T}-\nu_{z}=1$. When the horizontal component of the geomagnetic field was canceled, this attenuation was eliminated. The disturbance at $12.6 \mathrm{in.}$ was thought to be the sum-coupling resonance $\nu_{r}+\nu_{z}=2$, and this was verified as described later.

To identify the resonances uniquely, advantage was made of the fact that large changes in the axial focusing frequency can be produced by small changes in the amplitude of the azimuthal variation in the magnetic field; the radial focusing frequency is practically unaffected. The azimuthal variation was modified by changing the sector-coil current. The cyclotron was operated at $97,98.5$, $100,102.5$, and $105 \%$ of the standard sector-coil current settings. Each setting required a different set of currents for the 5 main circular coils and

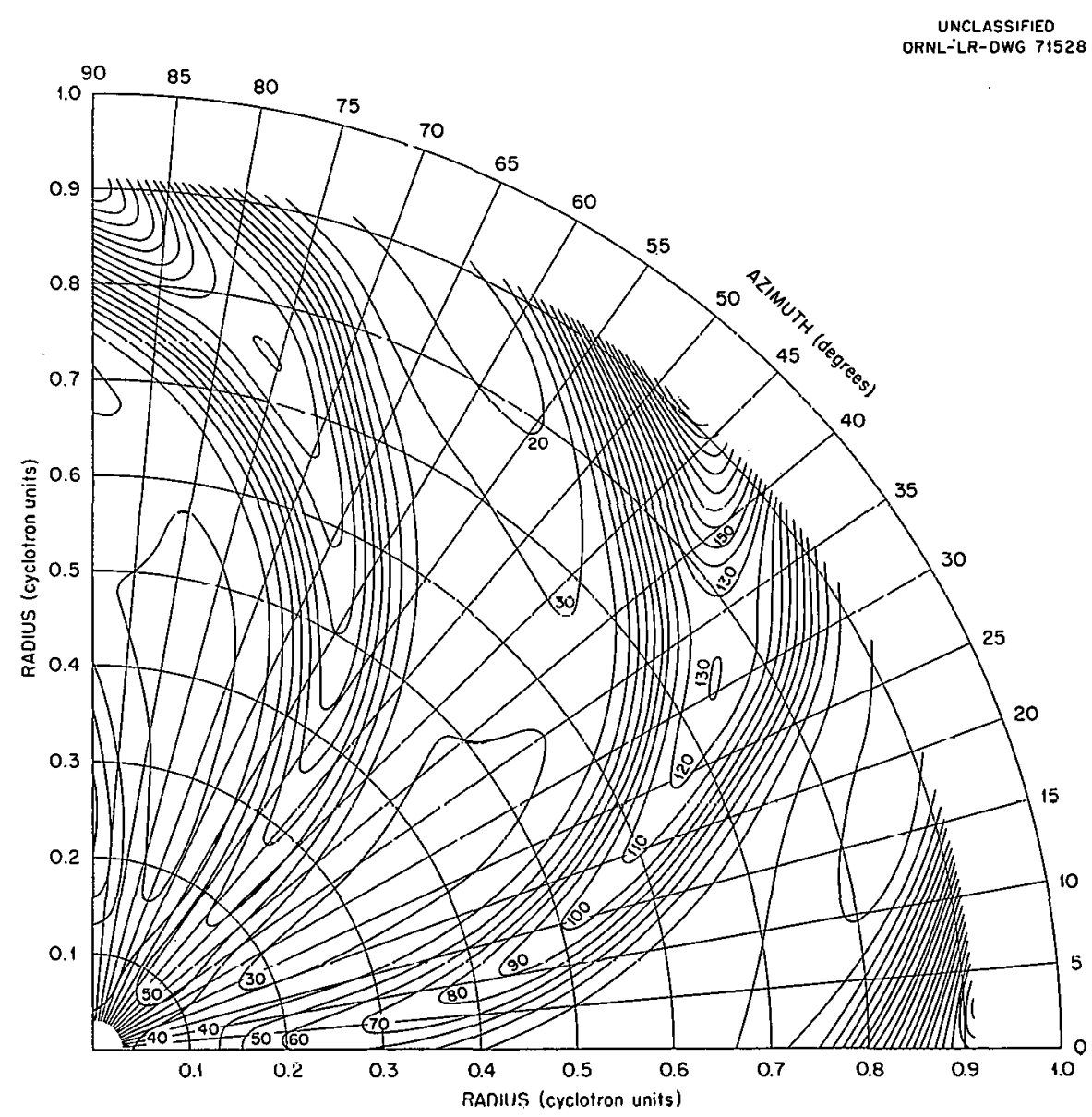

Fig. 5.1. Contour Map of One Quadrant of Eight-Sector Field of Analogue Magnet, in Gauss. 
the 32 trimming coils. These currents were derived by least-squares fitting to provide an isochronous mean magnetic field in each case. For the several conditions of operation, the locations of the resonances were recorded. These are indicated in Fig. 5.2 by bars which extend from the point where the attenuation or axial motion began to the point where the effect ceased. The solid lines are the computed equilibrium orbit properties for the several currents. The results show clearly that the movement of the inner and outer resonances is in accordance with $\nu_{r}-\nu_{z}=1$ and $\nu_{r}+\nu_{z}=2$ respectively.

\section{Attenuation}

A typical curve of the electron current as a function of radius, shown in Fig. 5.3, illustrates the severe attenuation that occurs at the difference-coupling resonance if the horizontal component of the earth's magnetic field is not compensated. This particular curve was taken with an electron source without an accelerating electrode, or slits, to collimate the beam.

The decrease in beam current beyond a radius of 10 in. has been the subject of much study. Gas scattering, lack of isochronism, and loss at reso- nances have been largely eliminated as causes. The most likely explanation appears to be the electron scattering from the tip of the probe, which results from the small radial width of the beam relative to the effective range of the electrons. At the 10-in. (150-kev) radius the range of the electrons in the aluminum probe is about $0.1 \mathrm{~mm}$ and the radial width of the beam is about $0.3 \mathrm{~mm}$. At $450 \mathrm{kev}$ the radial width decreases to $0.1 \mathrm{~mm}$ and the range increases to about $0.55 \mathrm{~mm}$. At this much greater ratio of range to radial width, the probability of electrons leaving the probe tip after scattering through small angles is greatly enhanced.

Somc preliminary tests of this hypothesis were made by using probe tips of different materials. Attenuation curves taken with aluminum and tantalum probes under identical cyclotron operating conditions are given in Fig. 5.4. At low energies the tantalum shows much lower readings, in agreement with published back-scattering ratios, but at the highest energies the relative decrease is much lower. This is because the maximum range of electrons in tantalum is. less than that in aluminum by a factor of 6 . The ratio of beam width. to range for tantalum is 1 rather than 5.5 for aluminum; hence the fractional loss by smallangle scattering is much reduced.

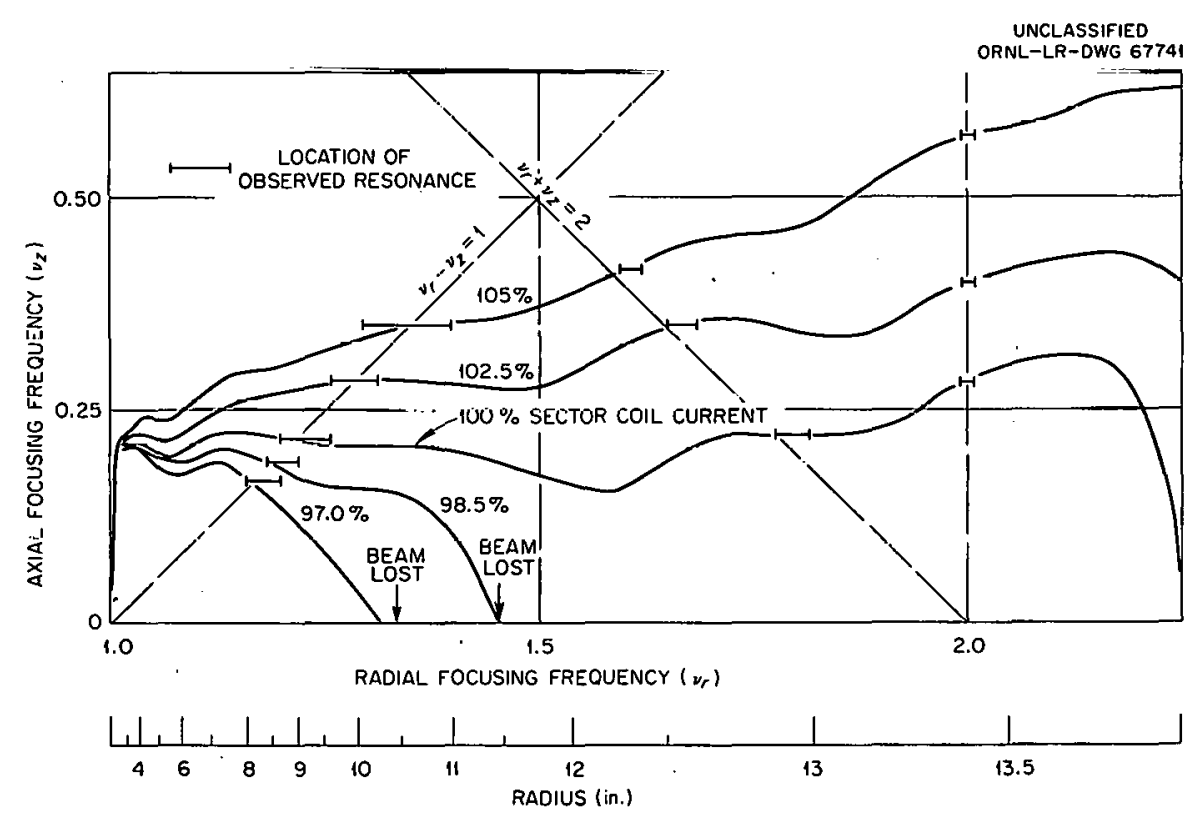

Fig. 5.2. Correlation of the Locotion of the Sum- and Difference-Coupling Resonances with. Sector-Coil Current. 


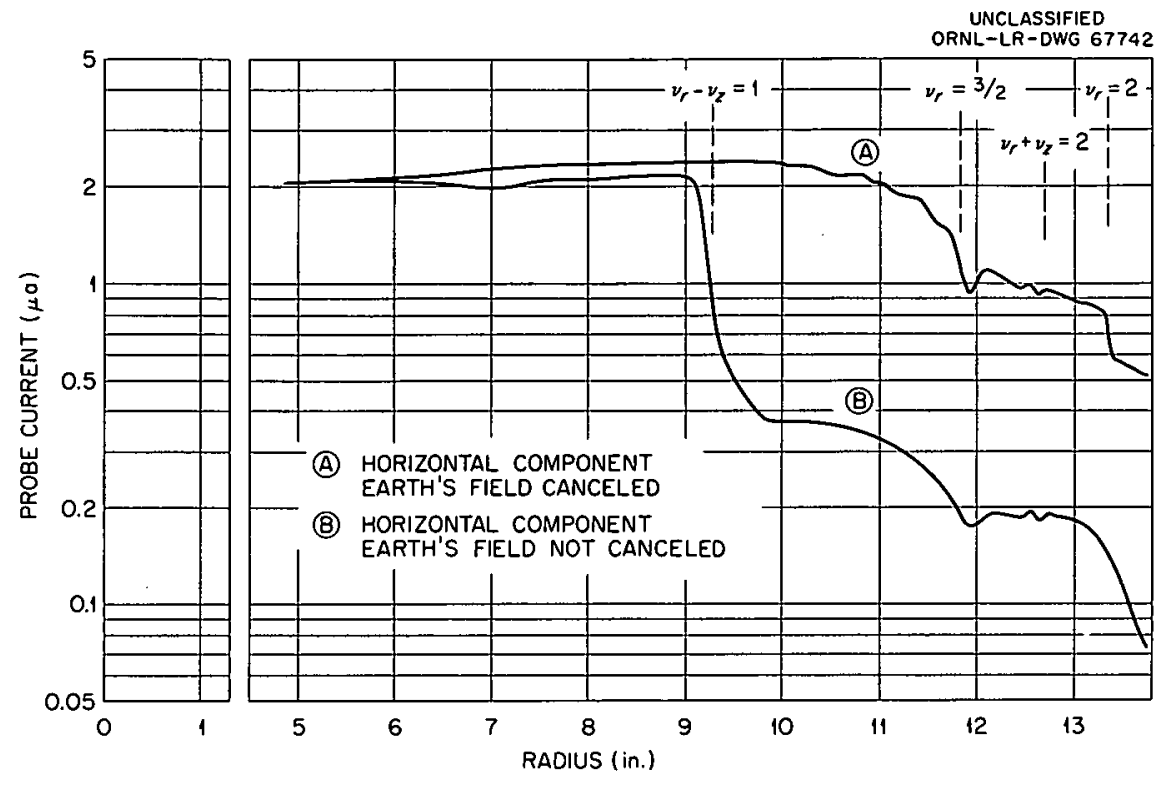

Fig. 5.3. Typical Attenuation Curves, Showing the Effect of the Difference-Coupling Resonance.

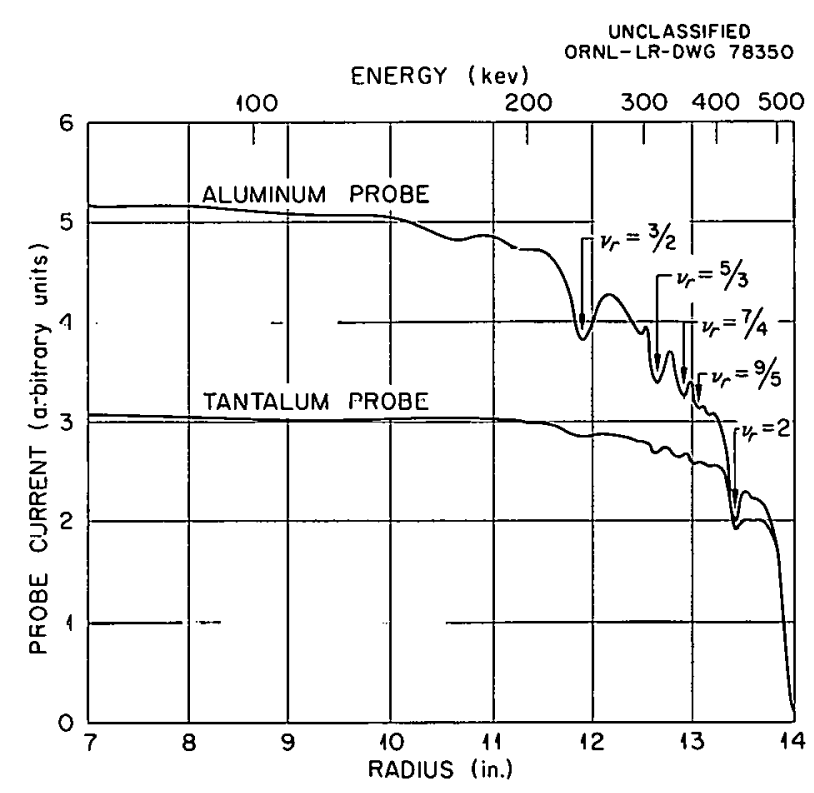

Fig. 5.4. Attenuation Curves Taken with Aluminum and Tantalum Probes.

The dips in beam current that occur at outer radii are located at points where the radial betatron frequency $\nu_{r}$ has the values $3 / 2,5 / 3,7 / 4$, and $9 / 5$. The decrease in current results from narrowing of a portion of the beam on the probe tip. At these values of $\nu_{r}$, a node in the betatron oscillation about the equilibrium orbit recurs after an integral number of revolutions; for example, at $\nu_{r}=3 / 2$, three complete oscillations occur in precisely two revolutions.

\section{Injection of Electrons}

The observations and measurements previously reported were obtained with a fixed filament mounted at the center of the cyclotron. In a later version the filament could be moved radially between the excited dee and the dummy dee to optimize its location for a given dee voltage. An attempt to improve the performance of this later system by the addition of an accelerating slit mounted on the lip of the excited dee was only moderately successful, chiefly because the accelerating slit was fixed and the system was difficult to optimize. Finally, an electron source was evolved which injects a highly collimated beam at an energy of $3.3 \mathrm{kev}$.

The electron injector, shown schematically in Fig. 5.5, is a two-stage device; the first stage defines the beam, and the second adjusts the energy. This arrangement has the advantage of minimizing the size of the device without unduly sacrificing performance. Electrons from a heated filament are accelerated by a potential of about $600 \mathrm{v}$ through the first slit, which has an aperture of $4 \mathrm{~mm}$ axially by $1 \mathrm{~mm}$ radially. They then pass 


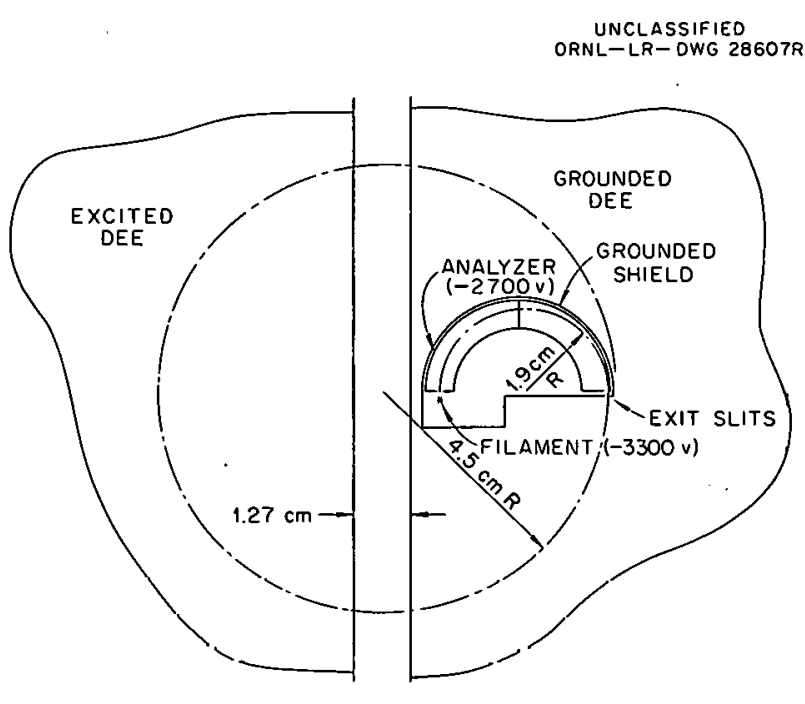

Fig. 5.5. Electron Injector.

through a $180^{\circ}$ spectrometer to an image slit of the same dimensions. At $90^{\circ}$ a $1-\mathrm{mm}$ slit defines the angular acceptance. As the electrons emerge from the image slit, they are accelerated through an additional $2700 \mathrm{v}$ so that their final energy is $3300 \mathrm{ev}$, which corresponds to an orbit radius of $45 \mathrm{~mm}$ in the central field of the Analogue. The assembly pivots about the exit slit so that the angle of injection may be adjusted.

Certain features of the performance of the Analogue are much improved by the injection of the highly defined beam. It is now possible to accelerate the beam through the difference-coupling resonance $\nu_{r}-\nu_{z}=1$ without attenuation, even without the horizontal magnetic field compensated; also, the beam accelerates through $\nu_{r}=2$, with only slight evidence of radial blowup. Finally, the results of the extraction studies which follow show that the radial amplitudes must be small enough to be practically unaffected by the $8 / 4$ resonance in the absence of the $\cos 2 \theta$ bump field. With the $\cos 2 \theta$ bump field the radial amplitude grows at two opposing hills only, which would not be the case if the beam entered the resonance region with large incoherent radial oscillation.

\section{Beam Extraction}

The beam-extraction system for the Analogue duplicates in principle the method proposed for the $\mathrm{Mc}^{2}$ cyclotron. The gain in radius per revolution of the particles at extraction radius is too small to permit entering of a magnetic or an electric channel, about $0.003 \mathrm{in.} /$ turn in the Analogue and about 0.050 in. for the $\mathrm{Mc}^{2}$ cyclotron. An additional mechanism is necessary to increase the turn separation at the extraction radius. The method used is based on the properties of the $8 / 4$ resonance $\left(\nu_{r}=2\right)$ which occurs at $445 \mathrm{kev}$ in the Analogue and at about $810 \mathrm{Mev}$ in the $\mathrm{Mc}^{2}$ cyclotron. It has been shown ${ }^{6}$ that at the $8 / 4$ resonance the change in radius per revolution is proportional to the cube of the amplitude; that is, $d r / d n=C\left(r-r_{0}\right)^{3}$. The value of the constant $C$ is about 200, so that for very small amplitudes, for example, $\left(r-r_{0}\right)=$ $10^{-3}, d r / d n$ is only $2 \times 10^{-7}$ cyclotron unit/turn. 'This illustrates the smalloamplirude srability of the resonance. To make the resonance useful for beam extraction, a small $\cos 2 \theta$ component is added to the magnetic field to destroy the smallamplitude stability by driving the $2 / 1$ resonance. The gain in amplitude per turn in the $2 / 1$ resonance is independent of initial amplitude, and the effect is sufficiently strong that only a few tenths percent of second harmonic relative to the mean field is required to develop enough amplitude so that the nonilinear resonance can increase the turn separation rapidly.

The maximum amplitude growth occurs at the hills in the magnetic field. This is illustrated by Fig. 5.6, which is a plot of the path of a particle on the sixth and seventh revolutions after an initial displacement of 0.01 cyclotron unit from the equilibrium orbit. The equilibrium orbits for 445 and $550 \mathrm{kev}$ are also plotted for reference.

After the turn separation of the beam has been amplified by the combination of the $\cos 2 \theta$ field perturbation and the nonlinear resonance, an additional mechanism must be provided to permit the particles to leave the cyclotron. The magnetic field must decrease sharply (in a magnetic: channel), or an electric force must be provided to counteract that of the existing magnetic field. In the Analogue an clectrostatic channcl is used.

The Analogue extraction mechanism, Fig. 5.7, consists of two 0.005-in.othick tantalum strips separated by a distance of about $\frac{1}{8}$ in. The inner of these (the septum) is grounded, whereas the outer (the deflector) is insulated so that several

\footnotetext{
${ }^{6}$ M. M. Gordon, "Sector-Focused Cyclotrons," p 234 in National Academy of Sciences - National Research Council Publication 656, 1959.
} 


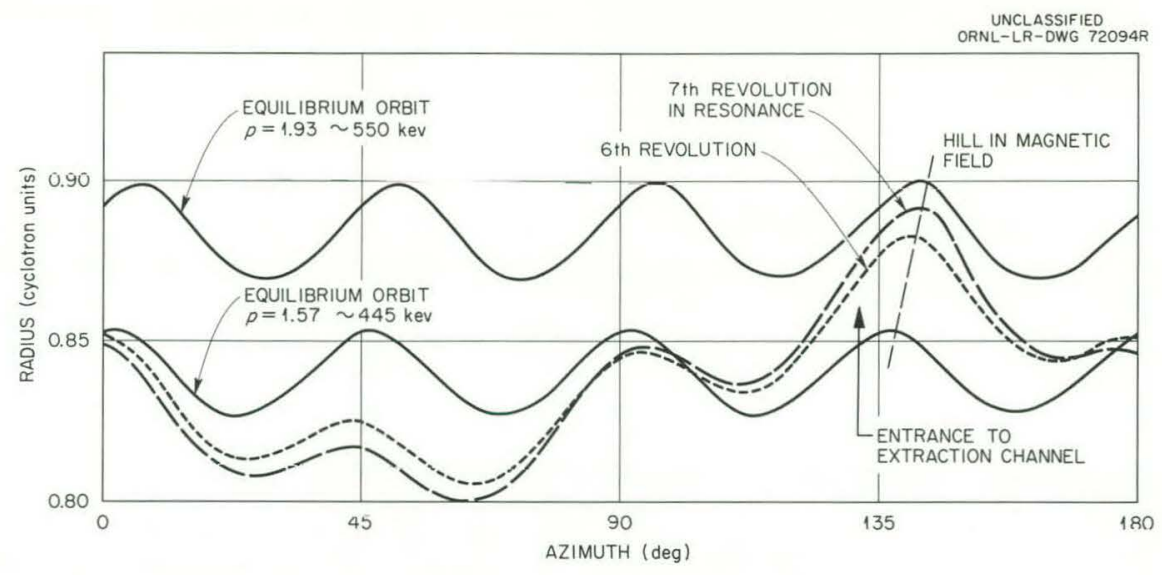

Fig. 5.6. Typical Particle Paths in the $8 / 4$ Resonance.

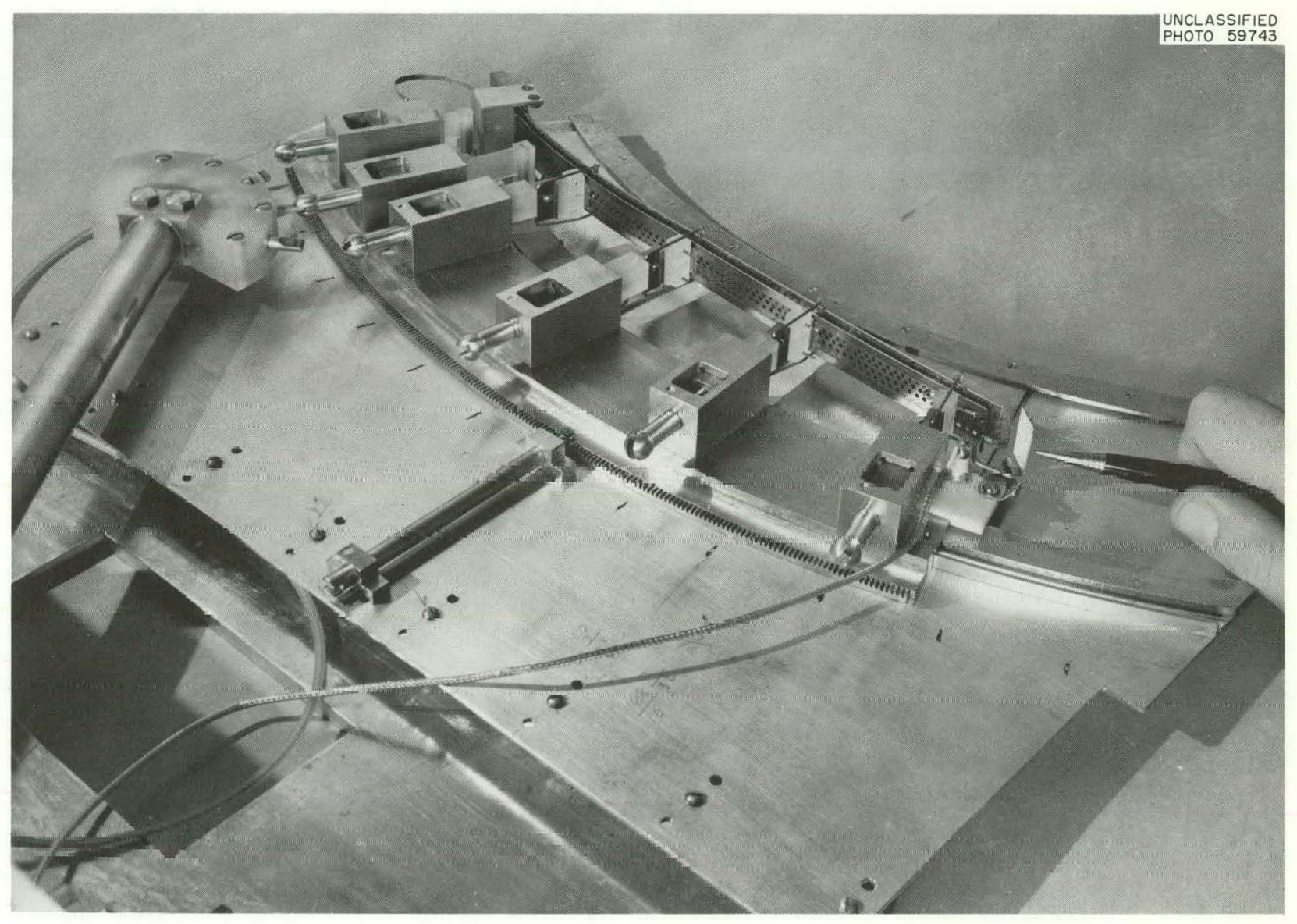

Fig. 5.7. Extraction Mechanism for Analogue Beam. Pencil indicates the exit. 
kilovolts may be applied. The channel is supported at five equidistant points, each of which can be moved radially by means of a screw adjusto ment to make the position of the channel match that required by the beam trajectory. An additional screw permits precise adjustment of the entrance angle of the channel without changing the entrance position.

Azimuthal positioning of the electrostatic channel assembly is by a rackeand-pinion mechanism. Movement of the several adjusting screws from outside the vacuum chamber is done with a multi。 headed screwdriver operated by a sealed shaft through a ball-and-socket vacuum seal on the faceplate.

The cus 20 magneticofield perturbation is prow vided by coils wound symmetrically to produce two hills and two valleys in the magnetic field without any effect on the average field. The coils are attached to the top and bottom lids of the vacuum chamber and are movable in azimuth to optimize the phase of the field perturbation.
To date the best extraction efficiency obtained has been about $85 \%$, with an extractor voltage of about $1.9 \mathrm{kv}$. This is a gradient of about $6 \mathrm{kv} / \mathrm{cm}$. The optimum $\cos 2 \theta$ amplitude is about $0.3 \%$ of the mean field. The beam impinging on a phosphorcoated target at the exit of the extraction system, Fig. 5.8 , produces a spot approximately $1 \times 5 \mathrm{~mm}$. This electric field $(6 \mathrm{kv} / \mathrm{cm})$ in the Analogue is equivalent to a magnetic channel strength of 3000 gauss for the $\mathrm{Mc}^{2}$ cyclotron.

The entrance of the extractor is about $8^{\circ}$ forward of the hill (see Fig. 5.6). To characterize the dependence of extraction efficiency on septum thickness for the present azimuthal location of the entrance, the septum thickness was increased stepwise by attaching metal-foil strips and measuring the extraction efficiency after each addition. The results, Fig. 5.9, illustrate several interesting features. First, the roughly linear relation between entrance thickness and extraction efficiency shows that the radial distribution of the beam entering the channel is approximately uni-

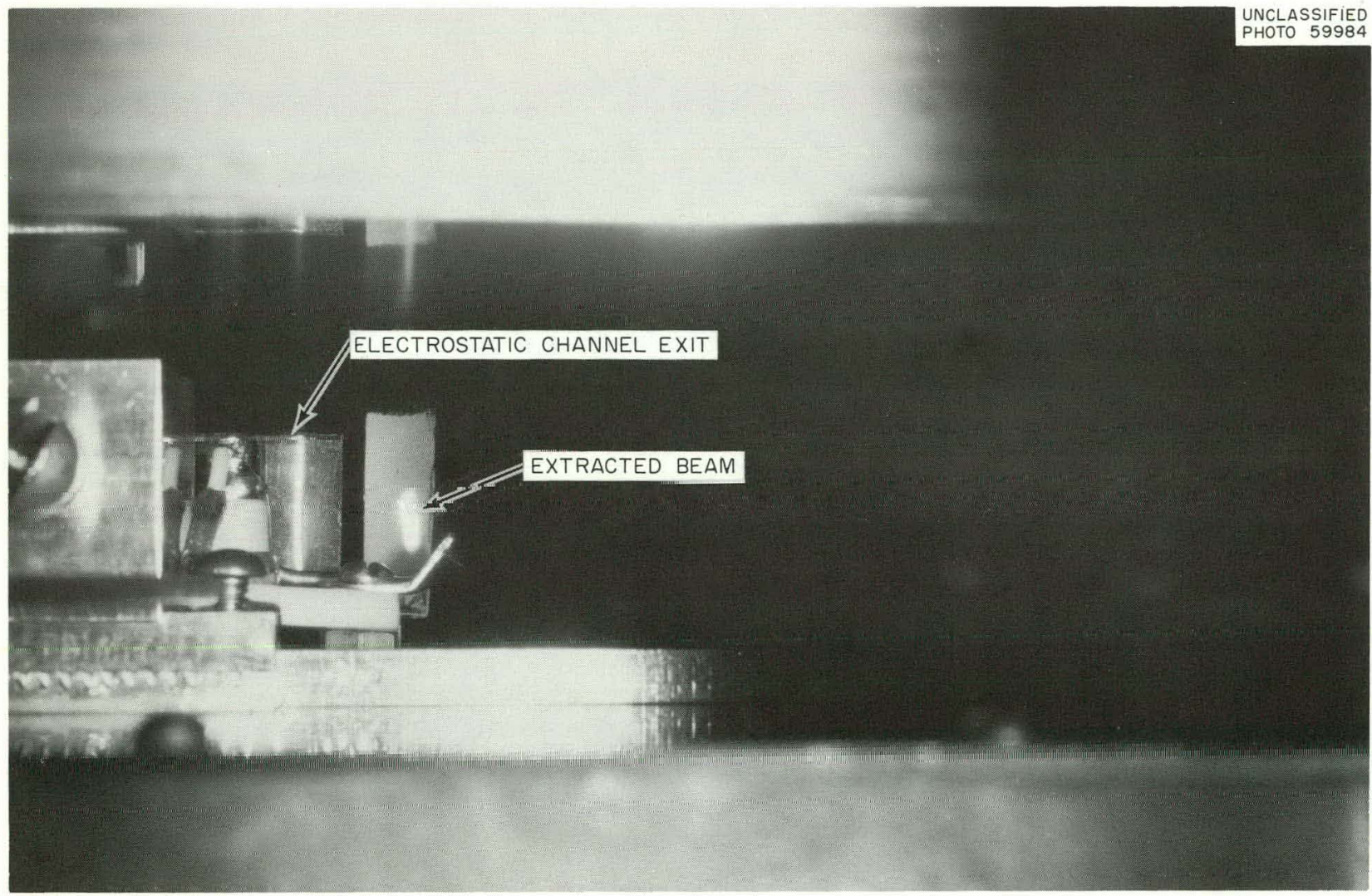

Fig. 5.8. Extracted Beam on a Phosphor-Coated Target. Actual size of the beam spot is $1 \times 4 \mathrm{~mm}$. 


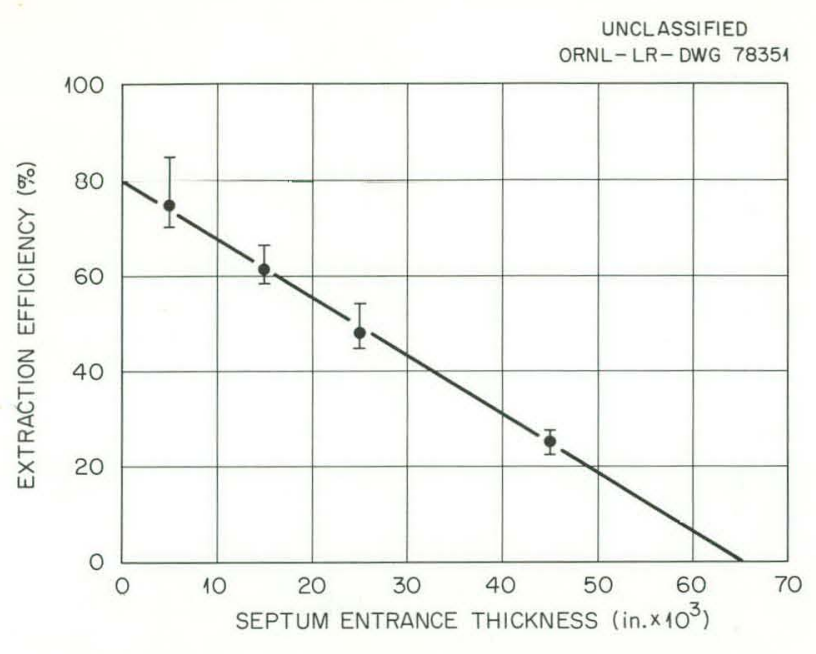

Fig. 5.9. Dependence of Extraction Efficiency on Septum Thickness.

form. Second, the efficiency does not extrapolate to $100 \%$ at zero entrance thickness, which suggests that the septum is improperly shaped at the entrance or that the entrance is effectively thicker because of the inherent angular distribution in the beam. Third, the efficiency extrapolates to zero at about one-half the channel aperture; therefore the beam does not fill the channel at the entrance. This is in agreement with Fig. 5.6. If we assume that the channel is filled at the point of maximum turn separation as a result of optimizing its shape, gradient, and the bump amplitude, then the beam width would be approximately a factor of 2 less at the channel entrance. It may be that higher efficiencies can be realized by moving the entrance of the channel nearer the point of maximum turn separation. Further experimental work, coupled with computer studies, will undoubtedly lead to a better understanding of the situation.

\section{THE Mc ${ }^{2}$ CYCLOTRON FACILITY}

Detailed investigations of many phases of the $\mathrm{Mc}^{2}$ Cyclotron Facility are being made in preparation for a final evaluation of its cost and feasibility. The status of these studies was described in a comprehensive special report. ${ }^{7}$ In the subse-

\footnotetext{
${ }^{7}$ The Mc ${ }^{2}$ Isochronous Cyclotron, ORNL-3324 (June $15,1962)$.
}

quent paragraphs the status at the close of this year is reviewed for the cyclotron design, building design, site consideration, magnet studies, rf cavity studies, and beam facilities design.

Other problems under intensive investigation but with results too incomplete to warrant reporting at this time are (1) an accurate estimate of the residual radioactivity associated with the cyclotron, (2) an improved design for the beam optics system for the experimental areas, (3) detailed plans for fabrication and assembly of the components of the machine, (4) the structurally sound design for the building to house the facility and staff, and (5) a detailed cost estimate for the whole project.

\section{Conceptual Design}

The $\mathrm{Mc}^{2}$ Cyclotron. - Since the last progress report, preliminary specifications for the cyclotron have been established (Table 5.1). These specifications will be revised as indicated by the magnet and rf-cavity studies now in progress. The arrangement of the eight spiraled magnet sectors with the coaxial if cavities, as presented in the previous report, is shown in Figs. 5.10 and 5.11. Systems for the installation, support, removal, and servicing of the magnet and rf cavity are under study.

The magnet support structure uses large columns with interconnected ring girders for rigidity. The columns penetrate the lower vacuum head and terminate on suitable foundations. An alignment system for individual sectors will be provided at the magnet support point in the chamber. A 150-ton overhead bridge crane will be used to assemble and service the machine. Owing to the large orbit separation at the center of the machine, stanchions can be used to space the magnet gap near the center and to prevent the large deflections of the sectors which would result from magnetic forces. When fully assembled, the overall height of the magnet yoke will be about $14 \mathrm{ft}$.

Because of the critical requirements on magnet alignment and the need for simplicity of construction, it now appears that the vacuum-tank system shown in Figs. 5.10 and 5.11 will best meet the requirements. With this system, both vertical and horizontal loadings due to atmospheric pressure are removed from the magnet. Since the vacuumchamber head is removable, the installation, alignment, and maintenance of the cyclotron components 
Table 5.1. Preliminary Design Specifications for the $\mathrm{Mc}^{2}$ Cyclotron

\begin{tabular}{|c|c|}
\hline $\begin{array}{l}\text { Proton energy, max (internal } \\
\text { beam) }\end{array}$ & $900 \mathrm{Mev}$ \\
\hline $\begin{array}{l}\text { Proton energy, extracted } \\
\text { beam }\end{array}$ & $810 \mathrm{Mev}$ \\
\hline $\begin{array}{l}\text { Mean orbit radius, extracted } \\
\text { beam }\end{array}$ & $230 \mathrm{in.}$ \\
\hline Number of sectors & 8 \\
\hline Spiral of sectors & $\theta=(r \omega / c)^{2}$ \\
\hline Tan $\gamma$, at extraction & 1.41 \\
\hline Central magnetic field & 4500 gauss \\
\hline Maximum magnetic field & 18,000 gauss \\
\hline Orbit frequency & $6.86 \mathrm{Mc} / \mathrm{sec}$ \\
\hline Beam space & 4 in. \\
\hline Magnet gap & 8 in. \\
\hline Magnet power & $10.0 \mathrm{Mw}$ \\
\hline Magnet weight, steel & 4800 tons \\
\hline Magnet weight, copper & 120 tons \\
\hline Accelerating system & 2 cavities, 4 gaps \\
\hline Radio-frequency $^{a}$ & 13.72 or $20.58 \mathrm{Mc} / \mathrm{sec}$ \\
\hline Energy gain, max & $1 \mathrm{Mev} /$ turn \\
\hline Radio-frequency power & 500 to $800 \mathrm{kw}$ \\
\hline Extraction system & $\begin{array}{l}\cos 2 \theta \text { magnetic bump at } \\
\nu_{r}=2 \text {, with magnetic } \\
\text { channel }\end{array}$ \\
\hline Extracted current & $100 \mu \mathrm{a}$ \\
\hline Vacuum volume & $2.6 \times 10^{6}$ liters \\
\hline
\end{tabular}

\footnotetext{
$a_{\text {Several cavity systems are being evaluated; they will }}$ operate on either the $2 \mathrm{~d}$ or the $3 \mathrm{~d}$ harmonic of the orbit frequency, depending on the system chosen.
}

are greatly facilitated. The vacuum tank will be $60 \mathrm{ft}$ in diameter and $50 \mathrm{ft}$ high. Preliminary investigation shows that, by using 30-in. T-beams for ring girders and 6 -in. vertical $T$-beams spaced $3^{\circ}$ apart, the tank wall can be fabricated from 0.5-in. plate stock. The dished heads will have a thickness of 0.5 in. except near the periphery, where they will be 0.75 in.

The design of the vacuum system is based on the desired operating pressure and the expected gas load. The operating pressure selected is $1 \times 10^{-6}$ torr. The gas load is determined by leaks, which can be minimized, and by outgassing, which is proportional to the total surface area and depends on the type of material exposed to the vacuum. Reasonably accurate estimates of the surface areas and types of materials can be made, but the wide variation in the published outgassing data makes it difficult to estimate with confidence the pumping speed required.

The previous estimate (last progress report) that 32 five-stage 32-in. diffusion pumps would be required was based on published outgassing data. The approach now being used is to determine the outgassing and surface areas of ORIC and then extrapolate to determine the pumping speed needed for the $\mathrm{Mc}^{2}$ cyclotron. These test data indicate that approximately 20 five-stage 32-in. diffusion pumps will be required to produce a pressure of $1 \times 10^{-6}$ torr in $10 \mathrm{hr}$.

The forevacuum system under study consists of three blowers in series-parallel arrangement, backed by threc oil-scalcd mechanical pumps. This system will evacuate the tank to 0.05 torr in about $1 \mathrm{hr}$ and $40 \mathrm{~min}$; the blower system would then be used to back the diffusion pumps. The speed of this backing system is sufficient to maintain a pressure of 0.1 torr at the outlet of the diffusion pumps while they are operating at maximum throughput.

Additional data will be taken from the ORIC vacuum system, and efforts will be continued to determine the most reliable outgassing data. As probable radiation intensities are determined, materials of construction and servicing methods will be studied. The many vacuum seals will require very careful consideration. Elastomer seals are preferred where possible. Undoubtedly, all-metal sealing techniques will prove prartiral for some applications.

The $\mathrm{Mc}^{2}$ Building. - Studies of the building requirements for the $\mathrm{Mc}^{2}$ facility led to the development of the conceptual design shown in Figs. 5.12 to 5.15. The key to the design is the large shielded area. The arrangement of this area is based on the planned physics program, with careful consideration for maximum versatility and possible future experiments.

The shielded area contains the cyclotron, the beam corridor, and three large experiment rooms. The cyclotron is housed in a large vault at one end of the building. Access to the cyclotron for installation and servicing is through removable roof blocks or through a 6-ft-wide personnel maze. A 150-ton overhead bridge crane is provided for moving roof plugs and heavy machine components. Space in the cyclotron building is also provided for shops, utilities, and assembly or staging areas. 


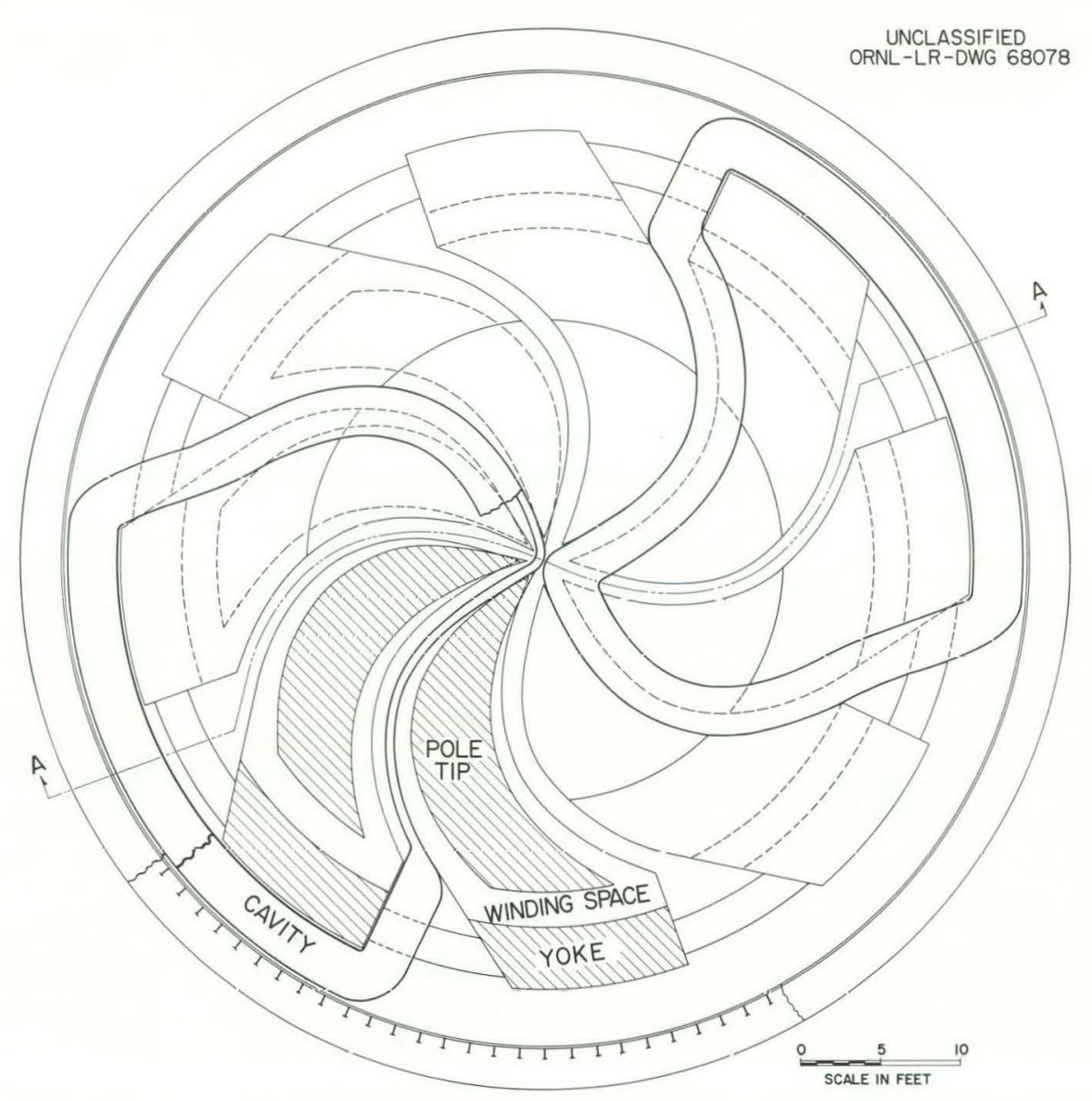

Fig. 5. 10. Plan View and Section of the $\mathrm{Mc}^{2}$ Cyclotron.

On the ground floor the three large experiment rooms and the beam corridor may be entered through large rolling-plug doors. These doors are large enough to permit passage of trucks for delivery or removal of equipment. Overhead bridge cranes in each room would be used for installation and servicing of experimental equipment. Considerable versatility in the arrangement of experiments is provided by full-length 6-ft-high slots (to be filled with removable blocks) in the walls between the beam corridor, the cyclotron, and the experiment rooms.

The mazes for personnel access to the experiment areas also serve as auxiliary utilities tunnels. Most of the shield penetrations for utilities will be cast into the concrete floor and shielding walls, thus leaving space in the mazes for any additional utilities that may be needed later.
Some changes may be required in the general layout of the shielded areas as a result of continuing studies of the ion-optics system. Also, structural design requirements relating to the large foundation loads may suggest certain changes in the layout.

Very thick shielding is required because of the intense proton beam capability of this cyclotron. This shielding represents a considerable part of the facility cost and therefore warrants very careful study. Preliminary studies indicate that, by using compacted, crushed limestone and earth in appropriate places, considerable savings can be effected. This feature will be studied further in conjunction with the structural studies presently under way.

The results of a study of four possible roof designs for the 80-ft-wide experiment rooms indicate that an arched roof with an earth cover 


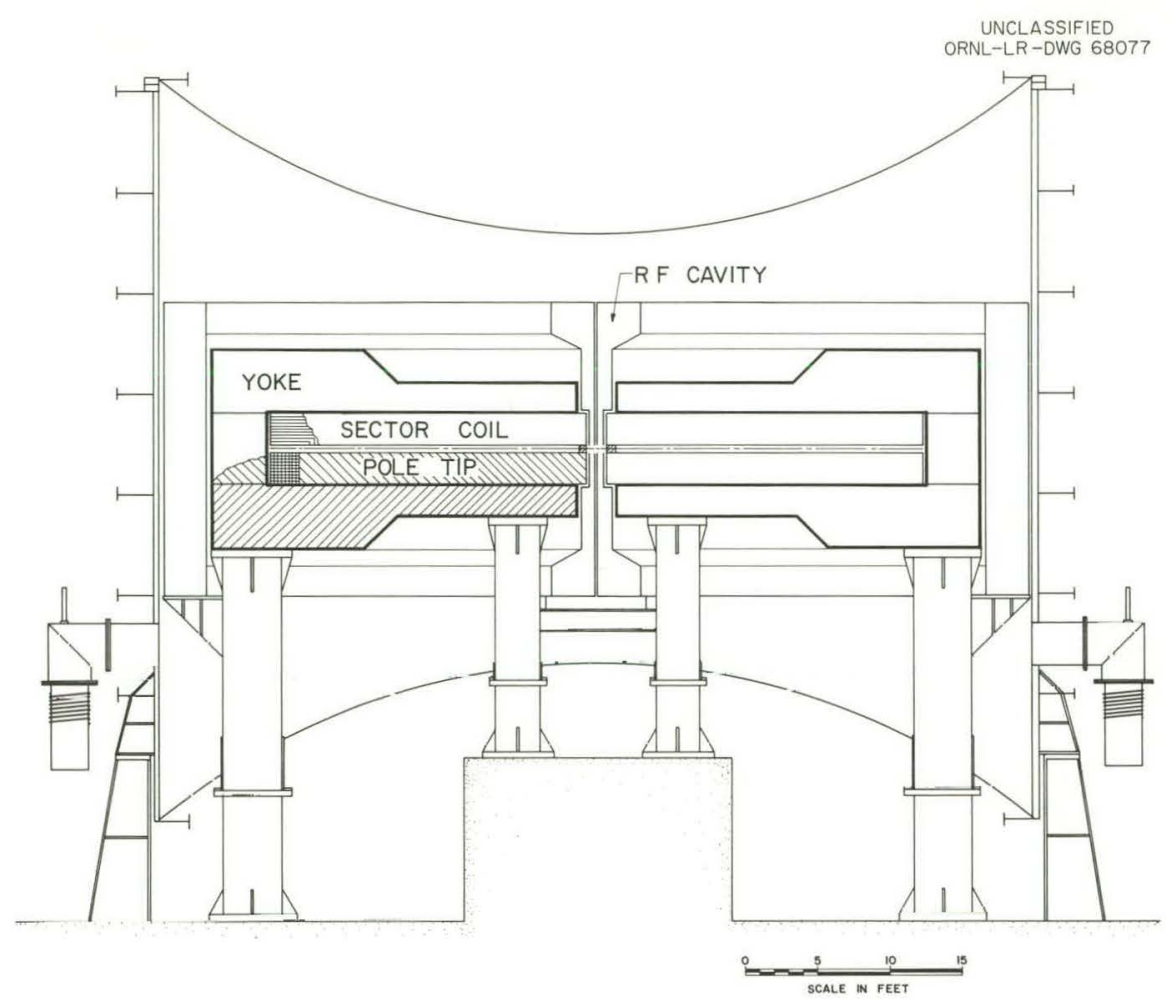

Fig. 5.11. Vertical Section of the $\mathrm{Mc}^{2}$ Cyclotron.

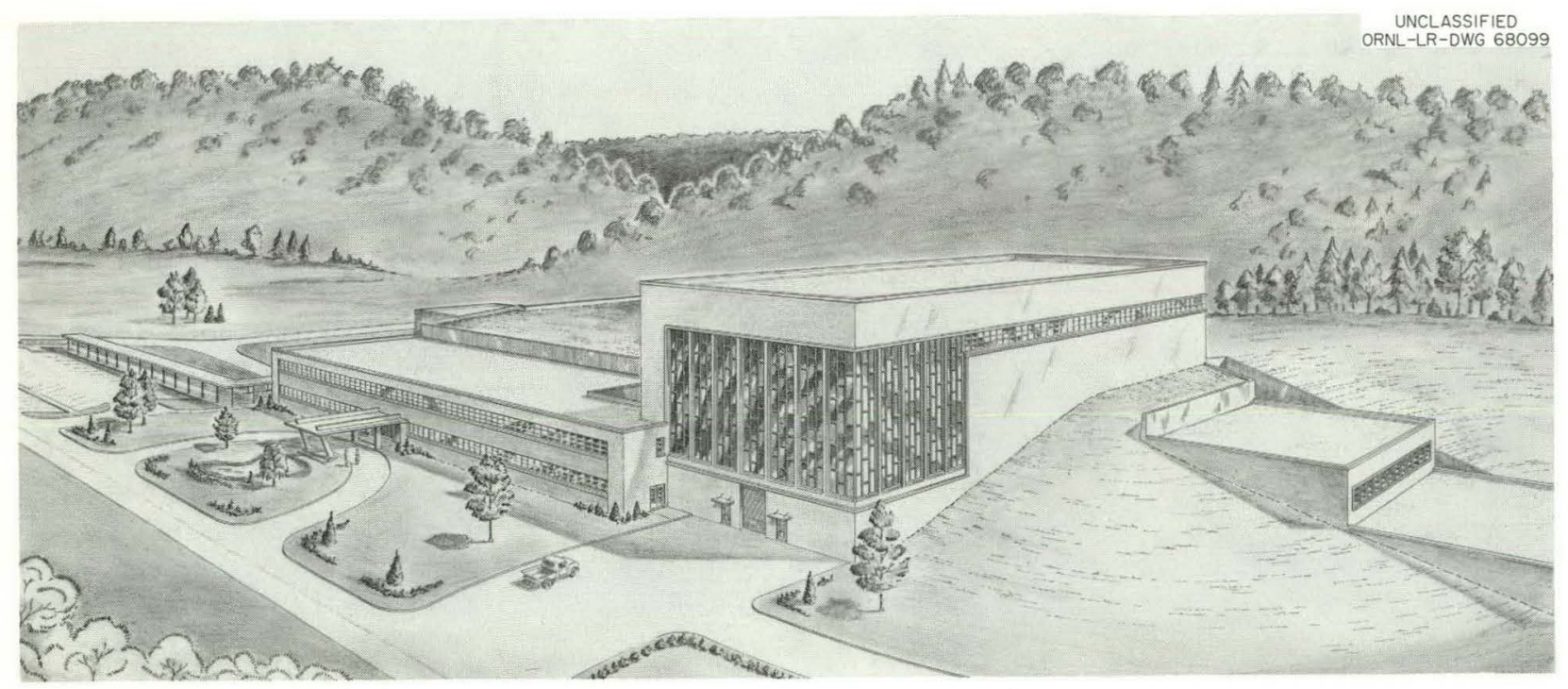

Fig. 5.12. $\mathrm{Mc}^{2}$ Cyclotron Facility. 


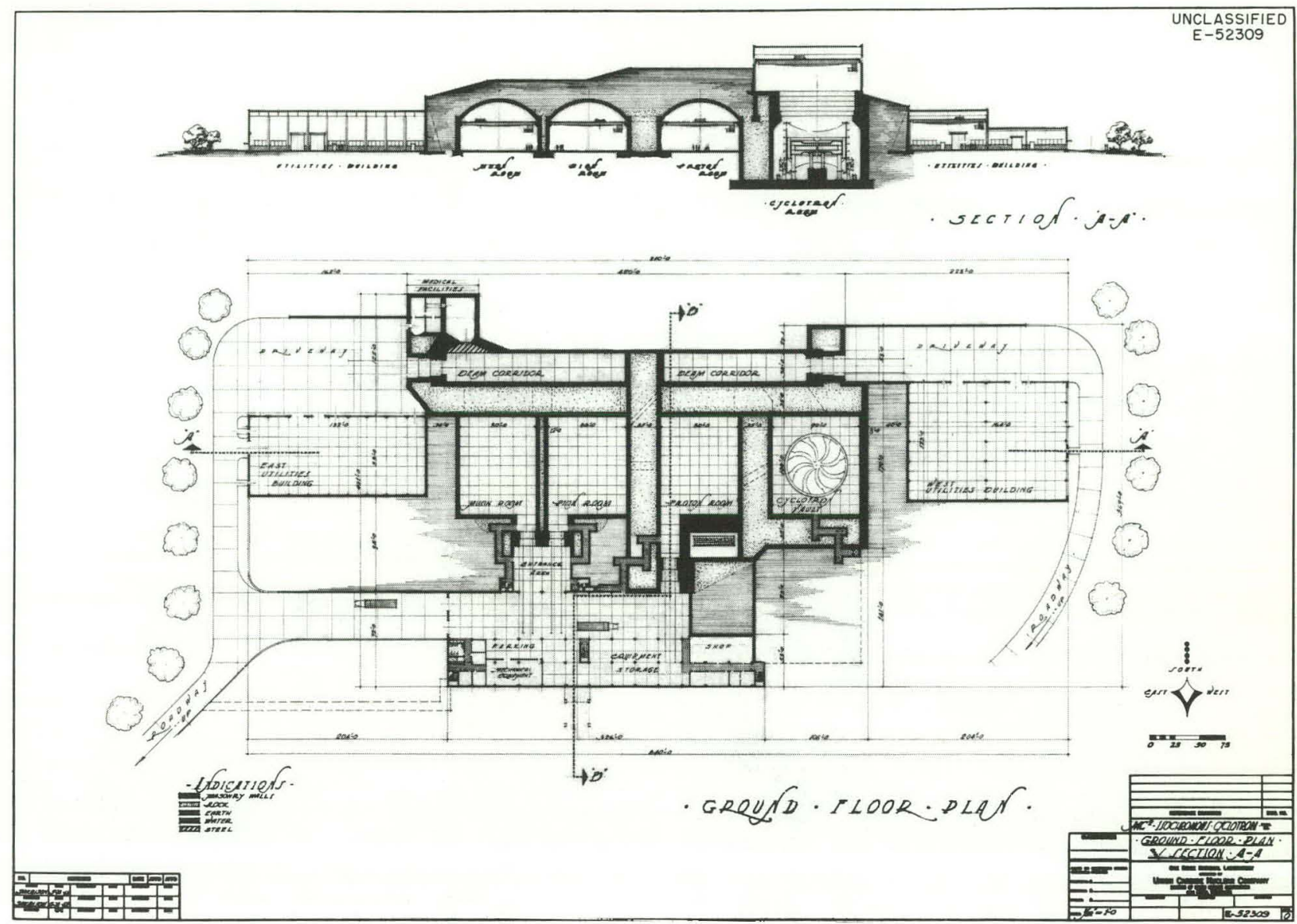

Fig. 5. 13. Ground-Floor Level of $\mathrm{Mc}^{2}$ Cyclotron Facility.

would be the most economical. The controlling cost factor in Fig. $5.16 a$ is the quantity of concrete, and in Fig. $5.16 b$ it is the reinforcing steel that would be required. The design shown in Fig. $5.16 c$ is lower in cost but is not as economical as that in Fig. 5.16d, which places the concrete in compression and, therefore, greatly reduces the amount of concrete and steel.

The office and laboratory building is placed adjacent to the experiment areas to provide a central headquarters for research and operating personnel. The building will be of conventional construction and will have three floors. On the ground floor, space is assigned for utilities, storage, and for the three plug doors in the open position. The second floor contains, in addition to office and laboratories, the cyclotron control center and counting room. This location provides for the shortest possible length of instrument and control wiring. The upper floor contains offices and laboratories.

A study of the structural problem of this building design is under way. This study has not progressed to the point that results can be reported at present. Problem areas appear to be the very high retaining walls, the large foundation loads, and the horizontal thrust of the arched roofs.

The $\mathrm{Mc}^{2}$ Site. - A tentative site has been selected for the facility. It is about $1500 \mathrm{ft}$ east of the main laboratory area and directly south of Building 6000 (see Fig. 5.17). This site is close to an existing road; therefore only a short approach road need be constructed. It provides a reasonably flat area about $2000 \mathrm{ft}$ long and $100 \mathrm{ft}$ wide (see Fig. 5.18). This area is parallel to a large hill and is of sufficient elevation to be well above the existing water table and to provide excellent drainage. The cyclotron is arranged so 


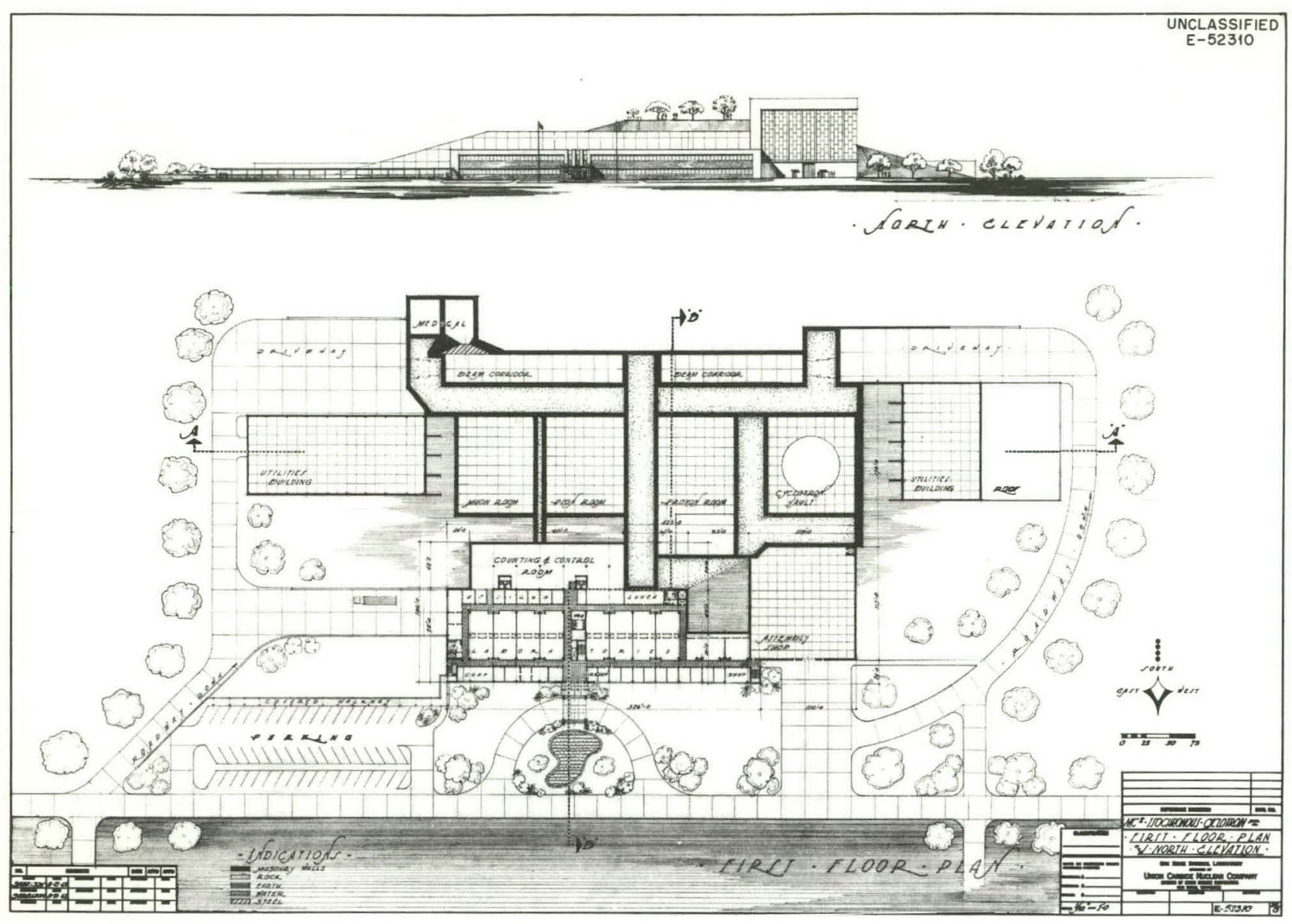

Fig. 5. 14. First-Floor Level of $\mathrm{Mc}^{2}$ Cyclotron Facility.

that the proton beam is directed east, away from the Laboratory. An open area extending about $1000 \mathrm{ft}$ beyond the end of the beam corridor is available for future expansion or experiments. Bus transportation to the main laboratory on an existing bus route will be convenient for the use of the existing facilities.

On the site selected, incomplete geological information indicates a limestone formation about $20 \mathrm{ft}$ below the surface. Core drillings will be made and evaluated to determine the underground rock structure in detail before a final site selection is made.

\section{Development Studies}

The two basic design requirements of the $\mathrm{Mc}^{2}$ cyclotron are (1) a method for producing a magnetic field of suitable focusing and isochronous properties and (2) a radio-frequency system with the appropriate frequency and with the desired electric gradient to accelerate the ions. Also, the design of the experimental facilities associated with the cyclotron depends basically on an ion-optics system for delivering beams of specified energy definition, purity, and intensity to locations where experiments can be performed. Some details of these basic studies are described below.

Magnet Studies. - The initial study of the magnetic-field requirements for the $\mathrm{Mc}^{2}$ cyclotron was concerned with developing a system that could generate the desired magnetic-field characteristics. At that time, relatively little consideration was given to minimizing power and steel requirements. This initial study led to a design that was incorporated in a 0.15 -scale model consisting of three of the eight sectors. Field measurements 


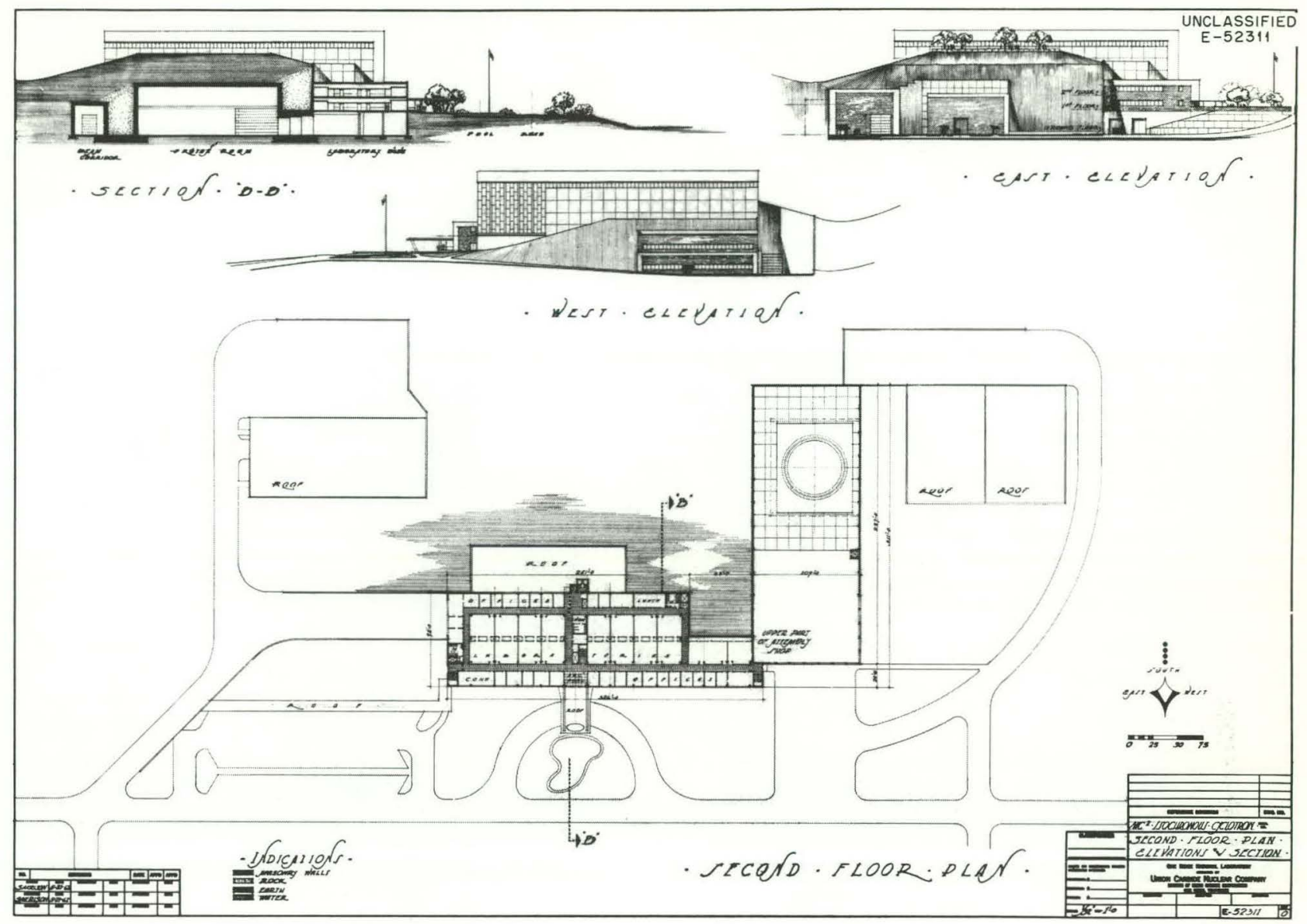

Fig. 5.15. Second-Floor Level of $\mathrm{Mc}^{2}$ Cyclotron Facility.

on the model verified that the magnetic-field configuration desired for the $\mathrm{Mc}^{2}$ can be readily generated in an iron-core magnet. In the model the field vs azimuth closely approximates a trapezoid (see Fig. 5.19), very much as was expected in the initial study.

The calculated and measured peak (hill) field vs radius, shown in Fig. 5.20, agree well within the accuracy of the measurements. The measured and calculated average fields vs radius are in equally good agreement (see Fig. 5.21). The measured and calculated flutter vs radius, Fig. 5.22, also have remarkable agreement at radii larger than 0.5 of maximum radius. At smaller radii the flutter in the model is about a factor of 2 larger than desired, because of the difficulty in accurately calculating the field in this region. Actually, the difference between the calculated and measured field shape is not very large. For example, at a 15 -in. model radius the flutter can be reduced to the desired value by widening the hill about $5^{\circ}$ and reducing the hill field a few percent to maintain the desired average field.

These data obtained with the model clearly demonstrate that this design is feasible for the $\mathrm{Mc}^{2}$ cyclotron if construction of the full-scale magnet directly follows the model coil construction; however, the power requirement would be about $16.5 \mathrm{Mw}$. By eliminating the extra leads, required on the model to obtain the desired ampere-turn distribution, and by more efficient use of the available volume for conductors, it is estimated that the power requirement for the large magnet can be held under 8 or $10 \mathrm{Mw}$. Some additional reduction in power may result from widening the gap between pole faces to allow more space for copper. This method of optimizing the design is under study. 


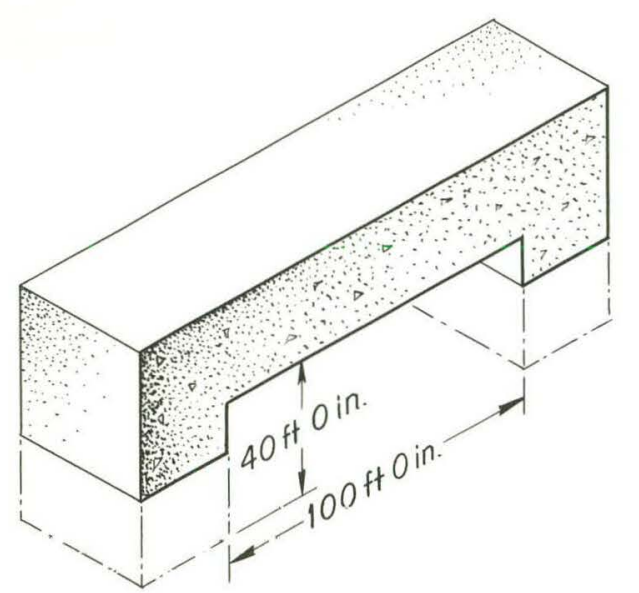

(a) CAST CONCRETE

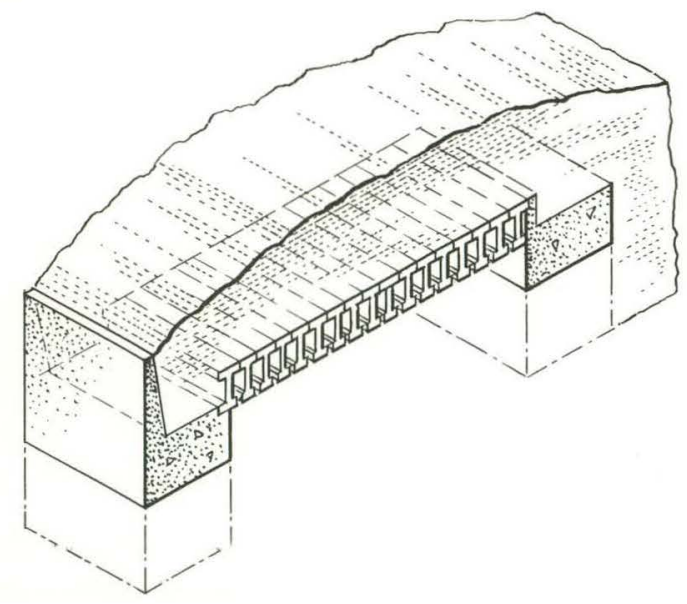

(c) PRECAST CONCRETE, WITH EARTH FILL

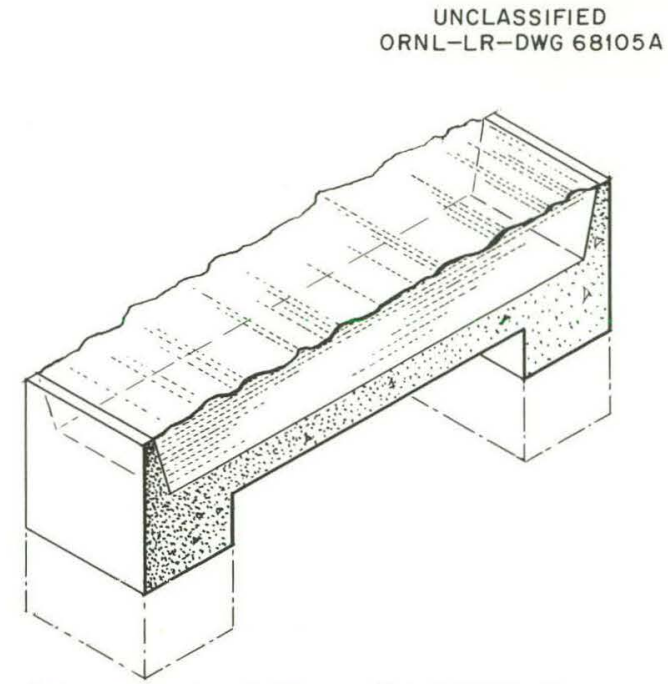

(b) CAST CONCRETE, WITH EARTH FILL

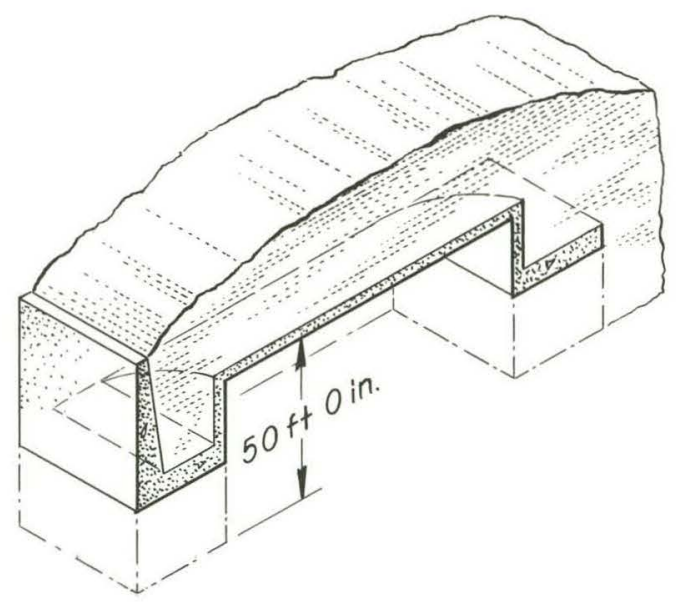

(d) CAST CONCRETE ARCH, WITH EARTH FILL

Fig. 5.16. Shielding Studies.

After the initial measurements were made on the 0.15 -scale model, the experimental program was directed toward investigating alternate designs for attaining the desired magnetic field. In one method studied, the gap was varied to produce the desired radial gradient; in another, the cross section of the yoke or pole was varied to utilize the saturation of the steel to produce the desired radial gradient. Considerable preliminary data were collected on these methods, and reasonable radial gradients were obtained. Radial gradients of over 1.3 kilogauss/in. (full scale) were obtained by sloping the pole-face surfaces in a model. The maximum gradients needed are 600 to 700 gauss/in. A very good approximation of the desired field vs radius for a 240 -in. cyclotron unit design is shown in Fig. 5.23. It should be noted that the field was obtained with pole tips having only two slopes. The gap varies from 5 in. at the 224-in. radius to $30 \mathrm{in}$. at the 174 -in. radius. The 30-in. gap near this radius is about the maximum that can be used and still obtain the required flutter. At smaller radii the gap must be reduced to a minimum, until near the center a 5-in. gap is wider than desired if flutter were the only criterion. There is little question that either of these methods or a combination of them can be used to design a magnet, but at this time 


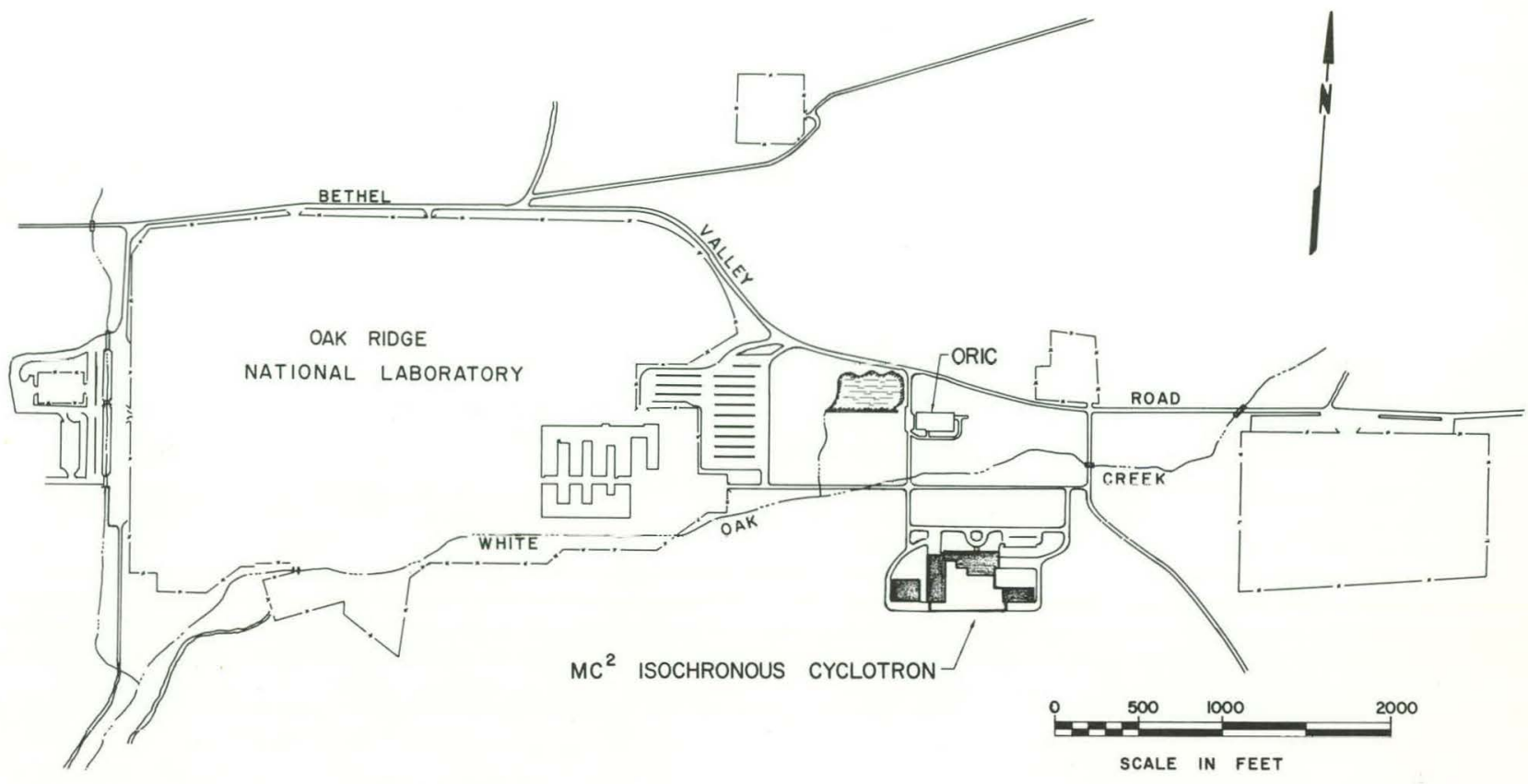

Fig. 5. 17. Proposed Site of the $\mathrm{Mc}^{2}$ Cyclotron with Respect to Other Laboratory Installations.

neither of the methods gives any promise of providing a significant reduction in power or steel, as compared with the method used for the initial 0.15 -scale model incorporating radial gradient coils.

A tentative conclusion is that a combination of methods will be used in the final design. Obtaining the magnetic-field gradients at the outside edge of the magnet by shaping the steel has the distinct advantage that the magnet coils may be removed from the gap, thus substantially reducing radiation damage to the coil insulation.

RF Cavity Tests. - The $\mathrm{Mc}^{2}$ cyclotron radiofrequency system will operate at a frequency of about $14 \mathrm{Mc} / \mathrm{sec}$, the second harmonic of the cyclotron frequency. With four accelerating gaps per revolution the maximum energy gain per turn will be $1 \mathrm{Mev}$. Cavity resonators rather than conventional dee structures will be used; cavities fitting between the magnet sectors occupy no magnet gap space and permit a more economical magnet design. Also, the excitation power is less for cavity resonators, and the gap voltage can be made reasonably constant with radius.
Either a pair of TE or a pair of coaxial (TEM) cavities is suitable for the $\mathrm{Mc}^{2}$ cyclotron. Models of these cavities were constructed and tested; they are shown in Fig. 5.24. The various characteristics of the models measured are given in Table 5.2. The large discrepancy between measured and theoretical $Q$ for the coaxial model is attributed to the pressure contact employed along the short-circuited ends and to the sharp corners around its periphery. The higher-order resonance modes observed in the coaxial cavity model produced voltage variations around the periphery of the cavity; they should present no problem, however, since they are at a considerably higher frequency than the desired mode.

A model of a "partial" or "auxiliary" cavity which could be used to increase the energy gain at larger radii was also tested. The configuration of this model and results of the tests are shown in Fig. 5.25.

The rf amplifier and coupling network for the $\mathrm{Mc}^{2}$ were examined briefly; one feasible system is shown in Fig. 5.26. The power amplifier consists of two RCA 6949 super-power triodes operating in push-pull; it should be capable of 


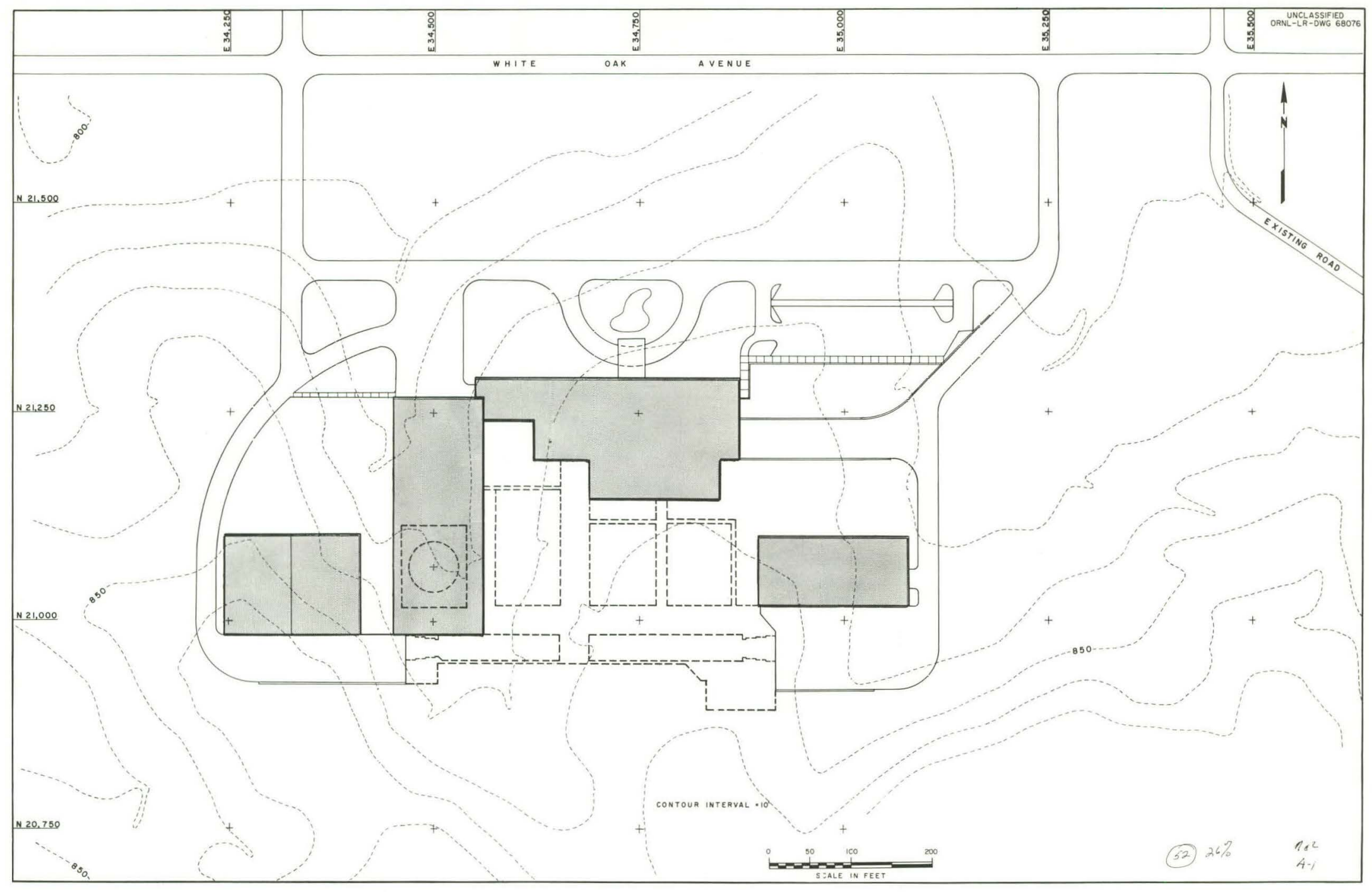

Fig 5. 18. Topography of Proposed Site. 


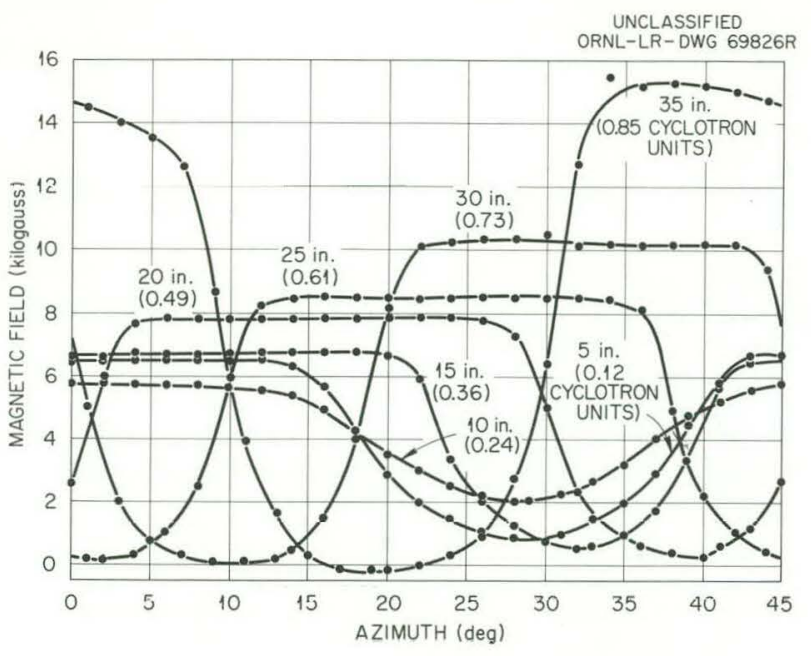

Fig. 5.19. Magnetic Field vs Azimuth in 0.15-Scale Model.

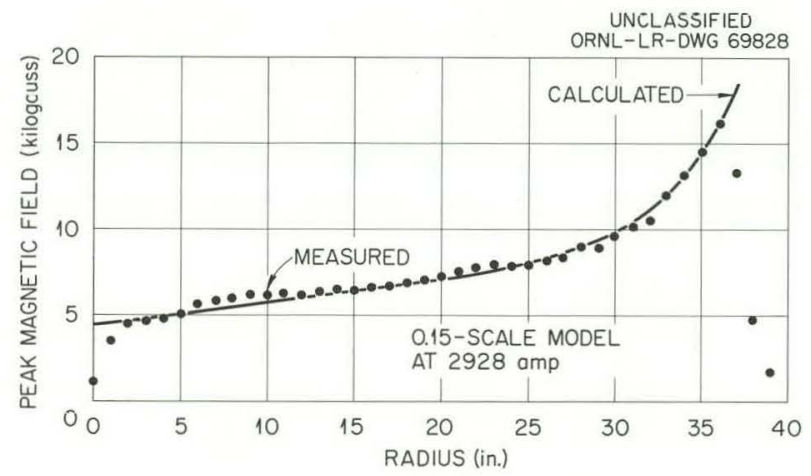

Fig. 5.20. Peak Field vs Radius.

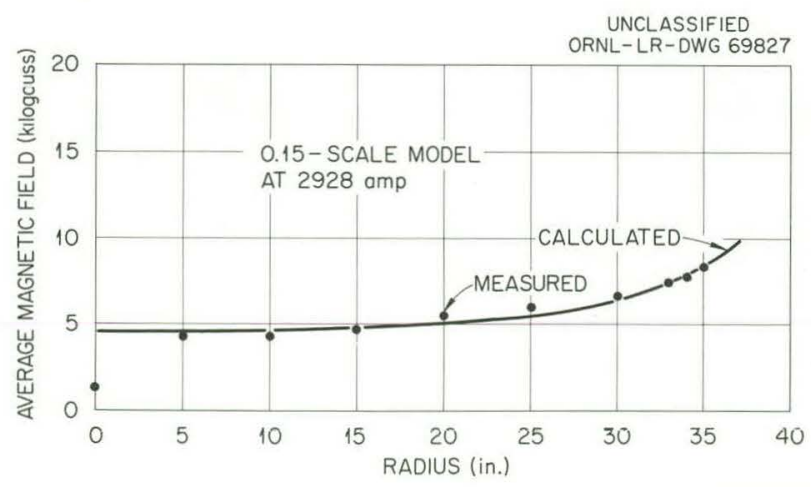

Fig. 5.21. Average Field vs Radius.

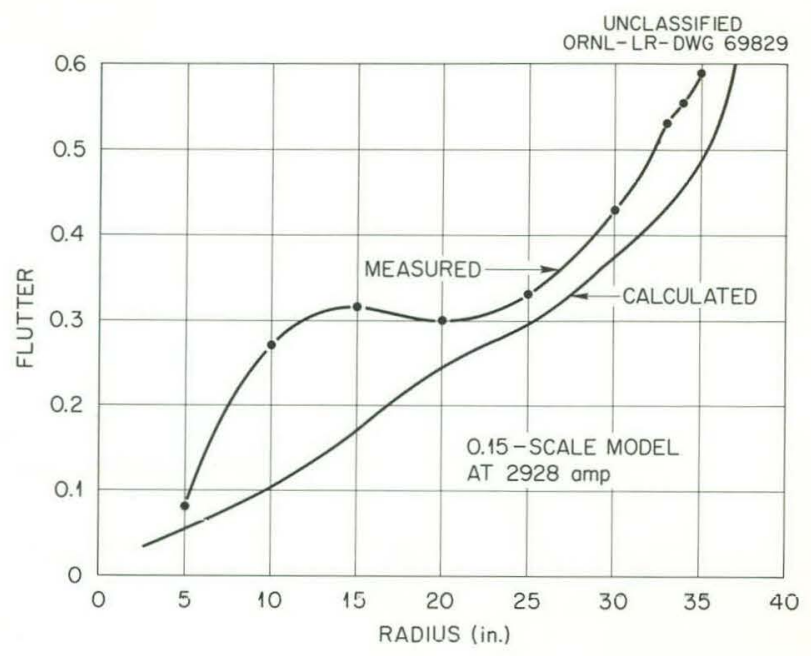

Fig. 5.22. Flutter vs Radius.

Table 5.2. Characteristics of Model rf Cavities

\begin{tabular}{lll}
\hline & TE Model & Coaxial Model \\
\hline Measured $Q$ & 4500 & 1800 \\
Theoretical $Q$ & 4500 & 4500 \\
Voltage variation & $\pm 5 \%$ min (func- & $\pm 5 \%$ \\
along gap & tion of tuning) & \\
Tuning range, Mc/sec & 149 to 160 & 139 to 152 \\
Observed resonances, & $158,253, \ldots$ & $152,193,220$, \\
Mc/sec & & $286, \ldots$ \\
\hline
\end{tabular}

supplying at least $800 \mathrm{kw}$ of $\mathrm{rf}$ power. Since the power amplifier would be located outside the cyclotron vault, the transmission line would be about 100 to $200 \mathrm{ft}$ long. If the line is matched to the load, then the length would be unimportant; it would be advantageous, however, to make the line an integral number of half wavelengths to ease the problem of sparking in the cavity. A driver-resonator model is being constructed to study the transient behavior of the system.

Facilities for the Muon Beam. - One of the main features of the ion-optics system proposed for the $\mathrm{Mc}^{2}$ cyclotron is the availability of a highintensity muon beam. The design of the facilities for such a beam must rely on the properties of the $\pi \rightarrow \mu$ decay characteristics. Pions with a 


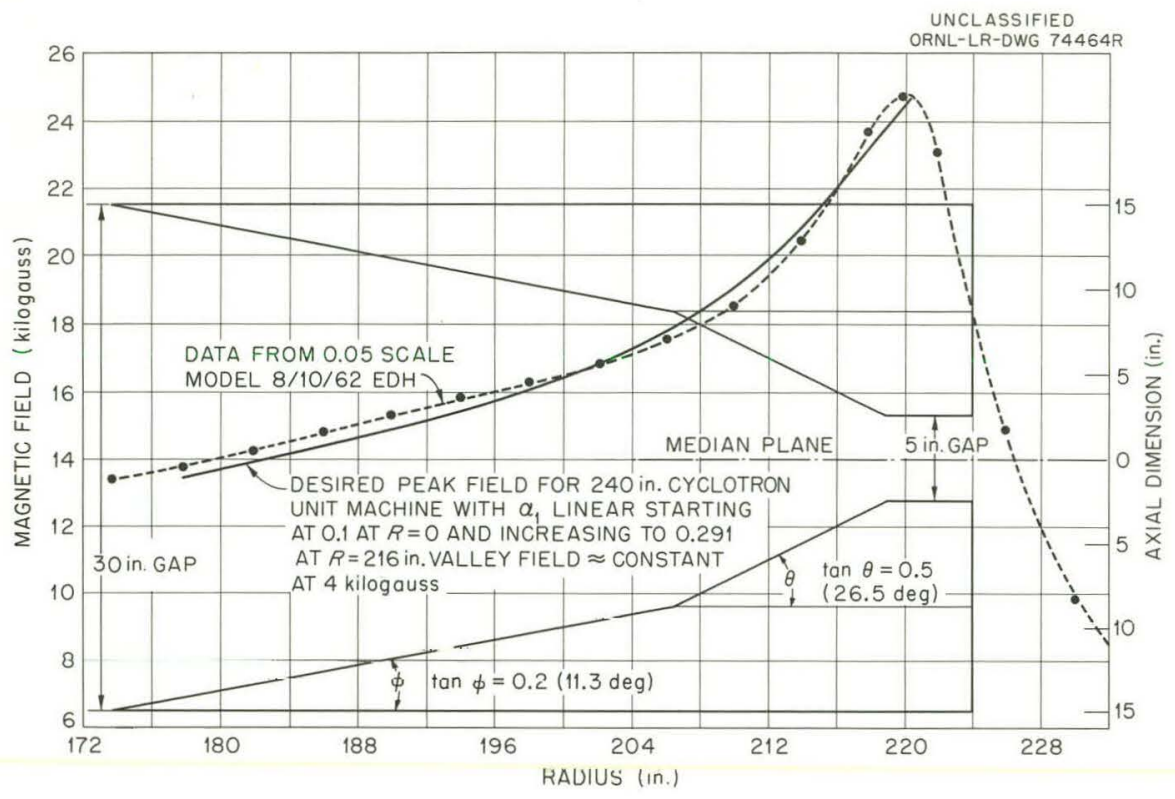

Fig. 5.23. Data from Model Magnet with Tapered Gap.

rest-frame mean life of $\tau_{\pi}=2.56 \times 10^{-8} \mathrm{sec}$ decay to muons. A pion of velocity $v_{\pi}$ will travel a mean distance of $v_{\pi} \gamma_{\pi} \tau_{\pi}$ before decaying, where $\gamma_{\pi}=\left(1-v_{\pi}^{2} / c^{2}\right)^{-1 / 2}$. Thus the mean flight path for pions of $420 \mathrm{Mev}$ kinetic energy (nearly the highest pion energy expected for the $\mathrm{Mc}^{2} \mathrm{cy}-$ clotron) would be about $100 \mathrm{ft}$.

In their own rest frame, pions give rise to monoenergetic muons with an isotropic probability distribution in space. When the pions decay while in motion, the angular distribution of emitted muons becomes peaked in the forward direction in the laboratory system; for sufficiently energetic pions, the muon distribution is restricted to lie within a cone about the pion direction. The half angle $\theta$ for the cone is given by

$$
\theta=\tan ^{-1} \frac{1}{\gamma_{\pi} \sqrt{\left(v_{\pi} / \nu_{\mu}^{\prime}\right)^{2}-1}},
$$

where $v_{\pi}$ is the pion velocity in the laboratory system, and $v_{\mu}^{\prime}$ is the velocity of the muon in the rest frame of the pion. The cone half angle $\theta$ is shown in Fig. 5.27 as a function of pion kinetic energy. This spread of the muon directions from the original pion direction, combined with the spread inherent in the original pion beam, produces a very divergent muon beam.

Another important feature of the $\pi \rightarrow \mu$ decay kinetics is that a monoenergetic beam of pions yiclds muons with an energy-independent distribution which extends from

$$
\left(\gamma_{\pi} \gamma_{\mu}^{\prime}-1\right) m_{\mu} c^{2}-v_{\pi} \gamma_{\pi} P_{\mu}^{\prime}
$$

to

$$
\left(\gamma_{\pi} \gamma_{\mu}^{\prime}-1\right) m_{\mu} c^{2}+{ }^{1}{ }_{\pi} \gamma_{\pi} P_{\mu}^{\prime},
$$

where $m_{\mu}$ is the muon rest mass and $P_{\mu}^{\prime}$ is the muon momentum in the rest frame of the pion. With this energy spread the pions and muons can be readily separated by momentum analysis.

Muon beams have been produced in two radically different arrangements at existing cyclotron facilities. The so-called "mu-meson channel" arrangement consists of a set of many identical and equally spaced quadrupole magnets. ${ }^{8}$ The channel is designed to trap and transmit muons arising from the in-flight decay of pions while

\footnotetext{
${ }^{8}$ A. Citron" et al., "A High-Intensity $\mu$-Meson Beam from the 600-Mev CERN Synchrocyclotron," Instrumen. tation for High-Energy Physics, Proceedings of an International Conference, p 268, Interscience, New York, 1961.
} 

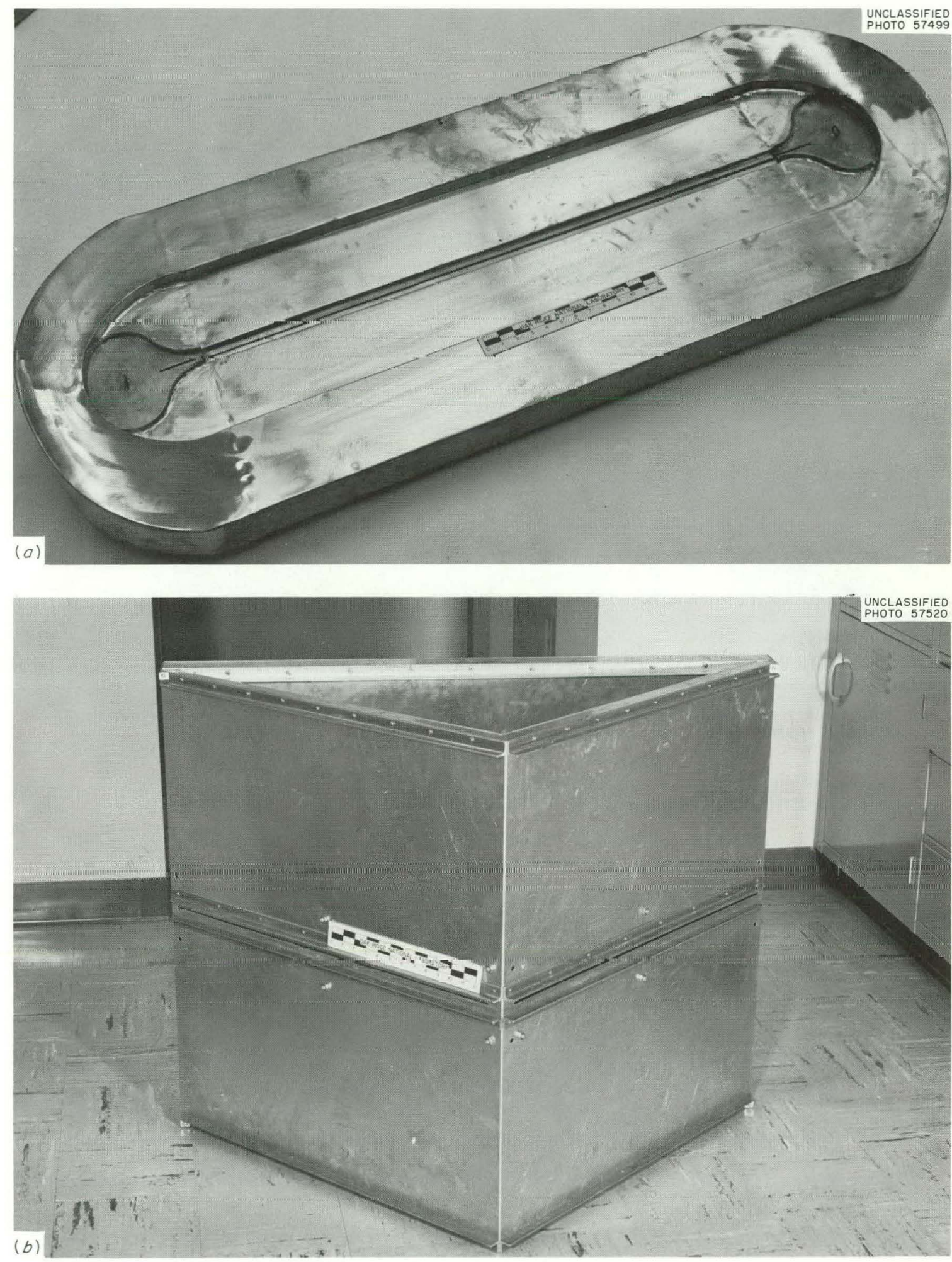

Fig. 5. 24. Model RF Cavitics. (a) TE cavity; (b) coaxial cavity. 

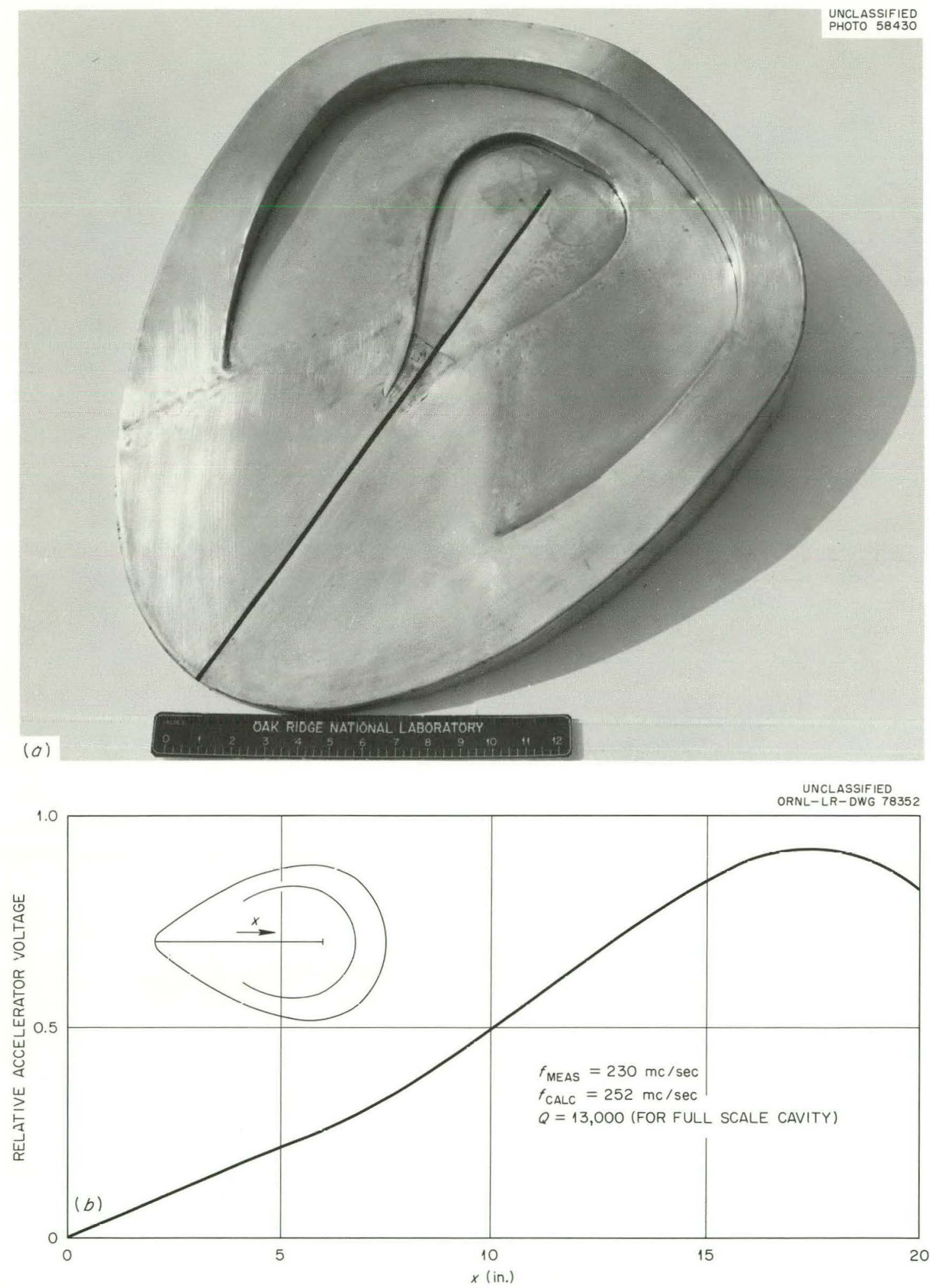

Fig. 5.25. Auxiliary Cavity and Voltage Distribution. (a) Auxiliary cavity, (b) test data. 
UNCLASSIFIED ORNL-LR-DWG 78353

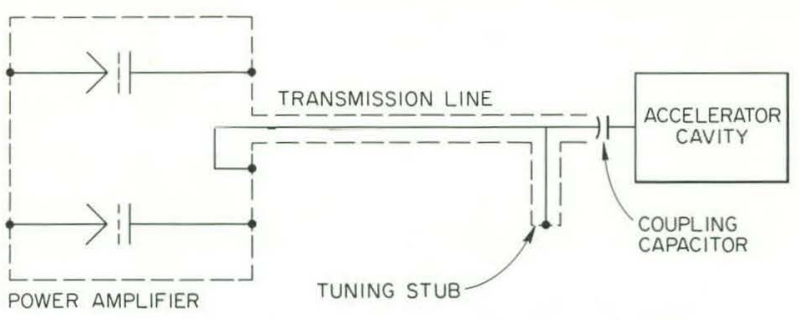

Fig. 5.26. Possible Power Amplifier and Coupling Network for the $\mathrm{Mc}^{2}$.

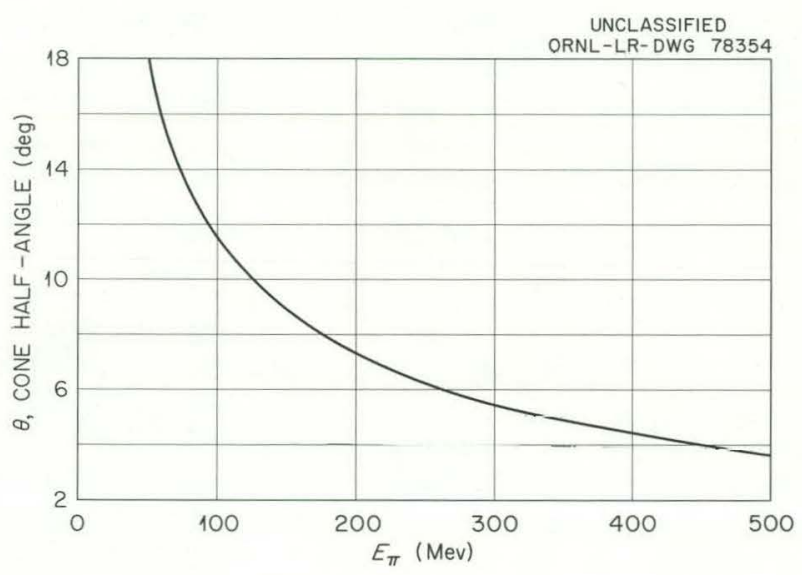

Fig. 5.27. Half Angle of Muon Cone as a Function of Kinetic Energy of Parent Pions.

they are traveling down the channel. As a result the channel has rather poor transmission properties with respect to particles produced at a point source before the entrance.

A different method for forming a muon beam uses a simple system of two quadrupole doublet lenses and a bending magnet. ${ }^{9}$ Operation of this system is based on collecting and focusing to an image the muons produced in the neighborhood of the pion source. Such a system can have a sizable transmission for particles produced near the source but essentially no trapping properties for particles produced by in-flight decay beyond the target. This system is also quite momentumselective and can be easily "tuned" to a variety of particle momenta.

${ }^{9}$ G. M. Bingham, Measurement of $G / M$ for Muons, UCRL-10107 (1962).
A precise comparison of the above two methods for producing muon beams is difficult because of different primary beam conditions, different targets, and different geometries. Roughly, the muon intensities available from the two facilities appear to be of the same order of magnitude; however, mu-meson channel design results in pion contamination of the muon beam which is several orders of magnitude less than that obtained in the two-lens system. To be sure, the latter beam could be further purified by various methods but at a sacrifice of muon intensity. Since a highpurity muon beam is necessary for many types of experiments, the channel design is preferred for a versatile muon facility.

\section{SEPARATED.ORBIT CYCLOTRON}

A preliminary study ${ }^{10}$ is being made of a new particle accelerator concept incorporating strong focusing and other attractive features. The major innovation in this machine is that the ions follow a spiral-helix orbit as indicated in Fig. 5.28.

${ }^{10}$ F. M. Russell, "A Fixed-Frequency, Fixed-Field, High-Energy Accelerator," Nuclear Instruments and Methods, in press.

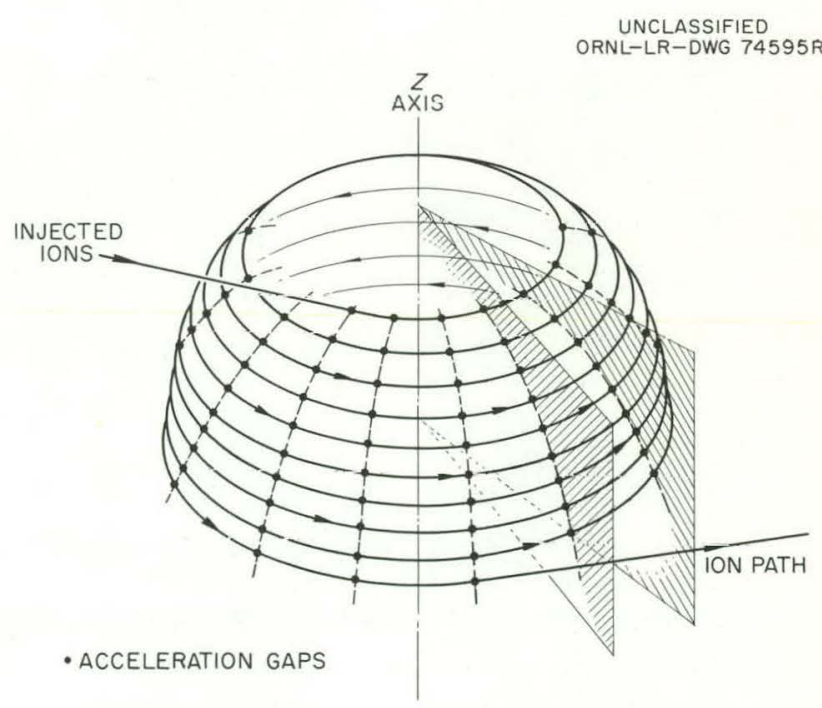

Fig. 5.28. Diagram of Beam Path with Both Radial and Axial Separation. Accelerating gaps are indicated by dots along the beam path. The rf voltages at the gaps joined by dashed lines are in phase. 
Acceleration is achieved by repeated passage of the ions through several rf cavity structures placed at regular angular intervals about the machine axis. The rf electric fields produced within these structures at the acceleration gaps are represented by dots in Fig. 5.28. The particles are

UNCLASSIFIED ORNL-LR-DWG 76872

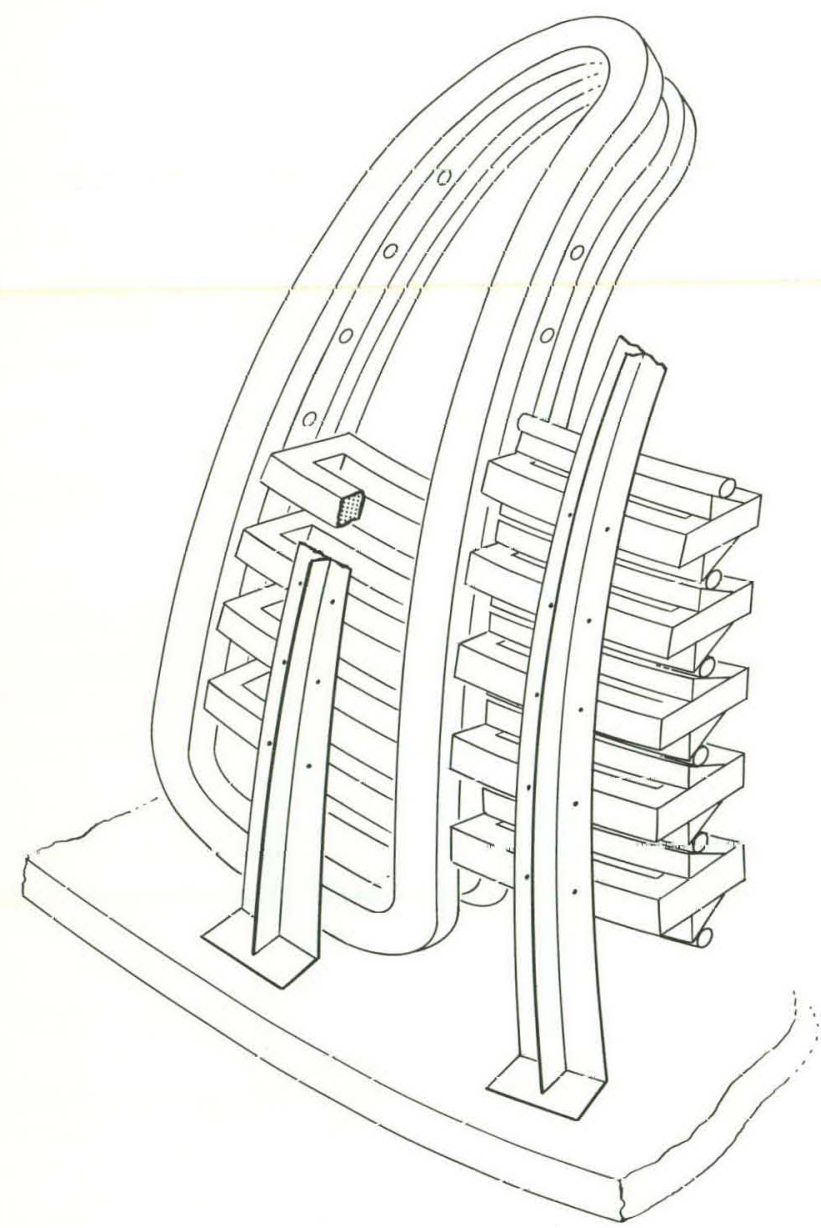

Fig. 5.29. Sketch of a Complete Magnet and RF Cavity Sector. Each sector contains one of cavity and pairs of magnet cells, the positive and negative gradient sections being mounted upon a common support so that the magnet pairs can be accurately aligned. The entire accelerator consists of $N$ such units, so arranged as to form a closed path. The slight changes required in each cell to allow for the increasing energy of the ions are obtained by placement of the shaped magnet shims on the pole faces. confined to oscillation about an equilibrium orbit by using alternating gradient components superposed on a steady magnetic field.

One method for generating such a magnetic field is indicated in Fig. 5.29. The main magnetic field is generated by coils individually wound on steel blocks and arranged in a near-vertical stack. In this way, vertically adjacent coils assist in generating the fields between the steel blocks. Tapered iron shims, mounted in pairs upon the blocks, produce the alternating gradients in the field. Figure 5.29 also indicates the relative positions of the magnet stacks and the rf cavities. The cavities consist of copper resonators operated in their lowest mode with the electric field parallel to the ion flight path. At the working frequency, ions take one-half an rf cycle in passing between surressive arreleration gaps. A sketch of such a cavity is shown in Fig. 5.30.

By choosing the appropriate energy gain per gap crossing and the mean strength of the magnetic field as a function of the energy of an ion, it is possible to make the period of rotation constant (isochronous).

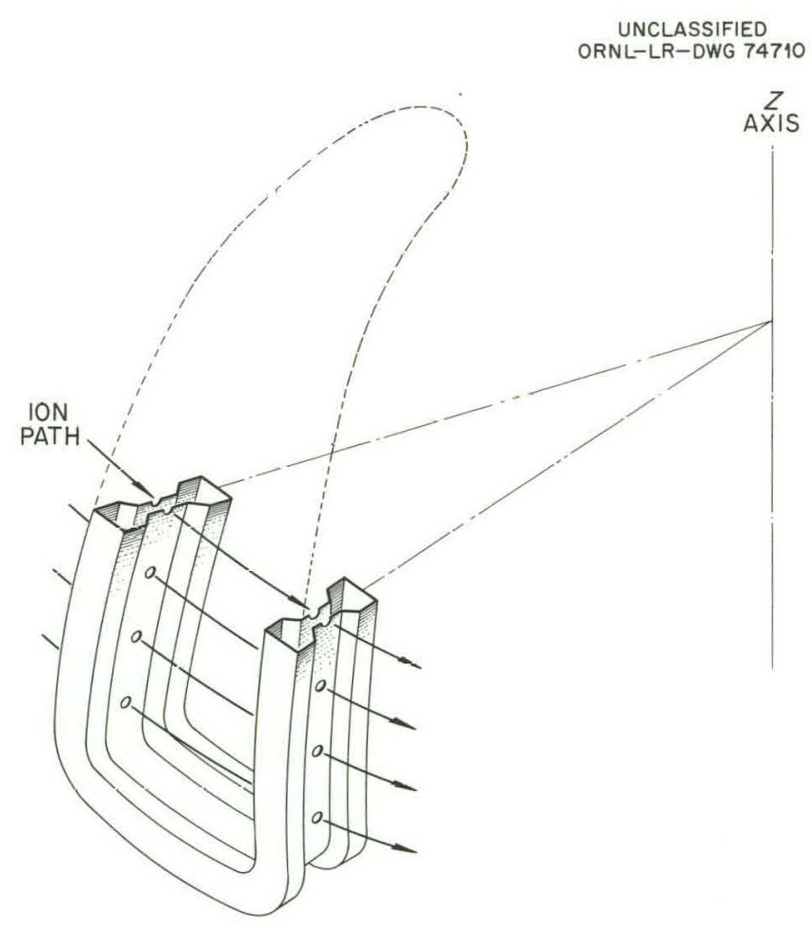

Fig. 5.30. Sketch of Cavity Showing Successive Passes of the Spiraled Ion Path. 
It can be shown that a machine of this type exhibits the following properties:

1. continuous beam output (as in a cyclotron),

2. high transverse stability (by strong-focusing action),

3. phase stability in longitudinal motion. (as in a synchrotron),

4. whole beam conveniently extracted (as in a linac),
5. output energy varied in steps (as in a linac), 6. no serious instability resonances,

7. good extracted beam quality.

A preliminary design study ${ }^{11}$ for such a machine to accelerate $1 \mathrm{ma}$ of protons to $1000 \mathrm{Mev}$ is being prepared.

\footnotetext{
${ }^{11}$ F. M. Russell, A Strong-Focusing Cyclotron with Separated Orbits, ORNL-3431, to be published.
} 


\section{The Oak Ridge Isochronous Cyclotron'}

\author{
R. J. Jones \\ R. S. Livingston \\ R. H. Bassel \\ R. S. Bender \\ B. Duelli ${ }^{2}$
}

\author{
M. P. Fricke ${ }^{3}$ \\ E. D. Hudson \\ R. S. Lord \\ M. B. Marshall \\ S. W. Mosko
}

\author{
E. G. Richardson, Jr. \\ A. Riikola \\ F. M. Russell ${ }^{4}$ \\ J. A. Russell ${ }^{5}$ \\ W. R. Smith
}

C. L. Viar
W. H. White, Jr.
R. E. Worsham
N. F. Ziegler
A. Zucker
The preliminary testing of the Oak Ridge Isochronous Cyclotron (ORIC) at full radius but at nominal power was completed. The power amplifier was then installed (see Fig. 6.1), and the machine is being readied for operation at full power.

The cyclotron is a 76-in., three-sector, azimuthally-varying-field, variable-energy machine. It is designed to accelerate various particles, within the $\mathrm{e} / \mathrm{m}$ ratio of 1 to 0.125 , to energies up to $145 \mathrm{Mev}$. Ample rf power has been provided to handle large beam currents, with 1 ma of protons at $75 \mathrm{Mev}$ being a typical design maximum.

Early this year a temporary rf system was installed so that the orbit dynamics of the marhine could be studied while the design and fabrication

\footnotetext{
${ }^{1}$ Several papers describing features of the Oak Ridge Is ochrumuus Cyclutron were presented at the International Conference on Sector-Focused Cyclotrons, Los Angeles, April 17-20, 1962, and published in the proceedings: R. H. Bassel, R. S. Bender, and J. G. Innes, "Analyzing Methods for ORIC Magnetic Fields," Nucl. Instr. Methods 18-19, 303 (1962); R. J. Jones, "Remarks on Tune-Up of Analogue II," Nucl. Instr. Methods 18-19, 469 (1962); R. J. Jones et al., "Unique Features in the Oak Ridge Isochronous Cyclotron," Nucl. Instr. Methods 18-19, 46 (19,62); R. S. Livingston ct al., "Concepts of an $\mathrm{Mc}^{2}$ Cyclotron," Nucl. Instr. Methods 18-19, 438 (1962); J. A. Martin and J. E. Mann, "Beam Characteristics in Cyclotron Analogue II," Nucl. Instr. Methods 18-19, 461 (1962); W. H. White, Jr., B. Duelli, and R. J. Jones, "Beam-Phase Measurement Studies," Nucl. Instr. Metbods 18-19, 601 (1962).

${ }^{2}$ On leave from the Kernforschungszentrum, Karlsruhe.

${ }^{3}$ Summer employee from the University of Minnesota.

${ }^{4}$ On leave from the Rutherford Laboratory, Harwell.

${ }^{5}$ Instrumentation and Controls Division.
}

of the permanent power amplifier were being completed. With this system a program of test operation at reduced dee voltage and beam energy simulated most of the problems of full-energy operation, but with more-tractable radiation conditions. Protons are held in phase for at least 500 revolutions, which is substantially in excess of the design value of 375 .

During the testing program the internal beam was successfully brought through an electrostatic deflector channel. The machine was then shut down, and a permanent high-power rf system was installed; final preparations are being made for testing at full power.

\section{ORIC MAGNETIC FIELD}

To achieve isochronous acceleration of ions over the large number of turns required in ORIC, it is necessary to maintain the radial gradient of the average magnetic field within a close tolerance. The shape of the radially increasing field is controlled by ten pairs of circular trimming coils (see Fig. 6.2). Three methods of achieving proper adjustment of the currents in these coils were used successfully: (1) the current settings were computed from the magnetic-field measurements; (2) the currents were adjusted while the magnitude of the beam current was monitored on a movablc probe; and (3) the currents were adjusted while the phase of the beam current was monitored on a movable probe specially developed for this purpose. The three basic methods can also be used in combination. The current settings obtained 


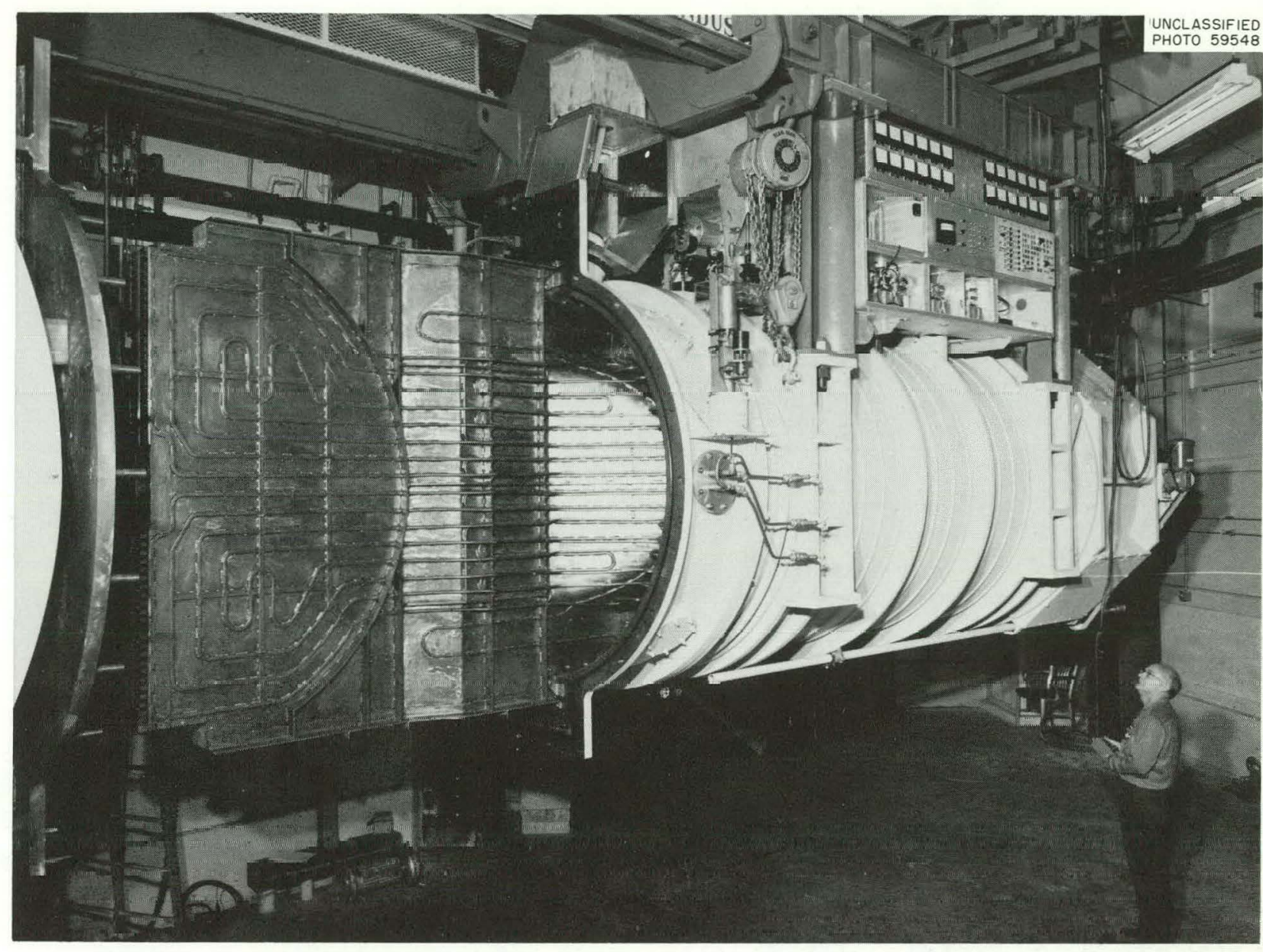

Fig. 6.1. Power Amplifier and Other RF Components Mounted on Top of the Dee-Stem Housing.

by several methods of optimization are shown in Table 6.1. The phase probe is a very effective aid in optimizing the magnet currents.

\section{DEFLECTION SYSTEM}

The deflection system includes four subassemblies placed within the cyclotron accelerating tank. The electrostatic channel performs the initial separation of the deflected beam from the circulating beam. During tests last June, approximately $30 \%$ of the circulating beam was brought through this channel, which consisted of a $1 / 16^{-i n .-}$ thick slotted-graphite septum and an uncooled aluminum deflector electrode, spaced $\frac{1}{2}$ to $\frac{5}{8}$ in.
Radioautographs of the circulating and deflected beams are shown in Fig. 6.3. Measurements of beam quality by radioautograph and by melted-foil techniques indicate that there is no large axial or radial divergence through the electrostatic deflector. A series of melted foils is shown in Fig. 6.4.

Following the deflector are the first and second sections of the magnetic channel, which reduce the magnetic field along the path of the deflected beam. After passing through the channels the beam enters the No. 1 beam-positioning magnet, which reinforces the fringing field to give the beam the proper angle for entrance into the external ion-optics system.

The first section of the magnetic channel is of "coaxial" design to minimize the field disturbance 
UNCLASSIFIED

ORNL-LR-DWG 58399R2

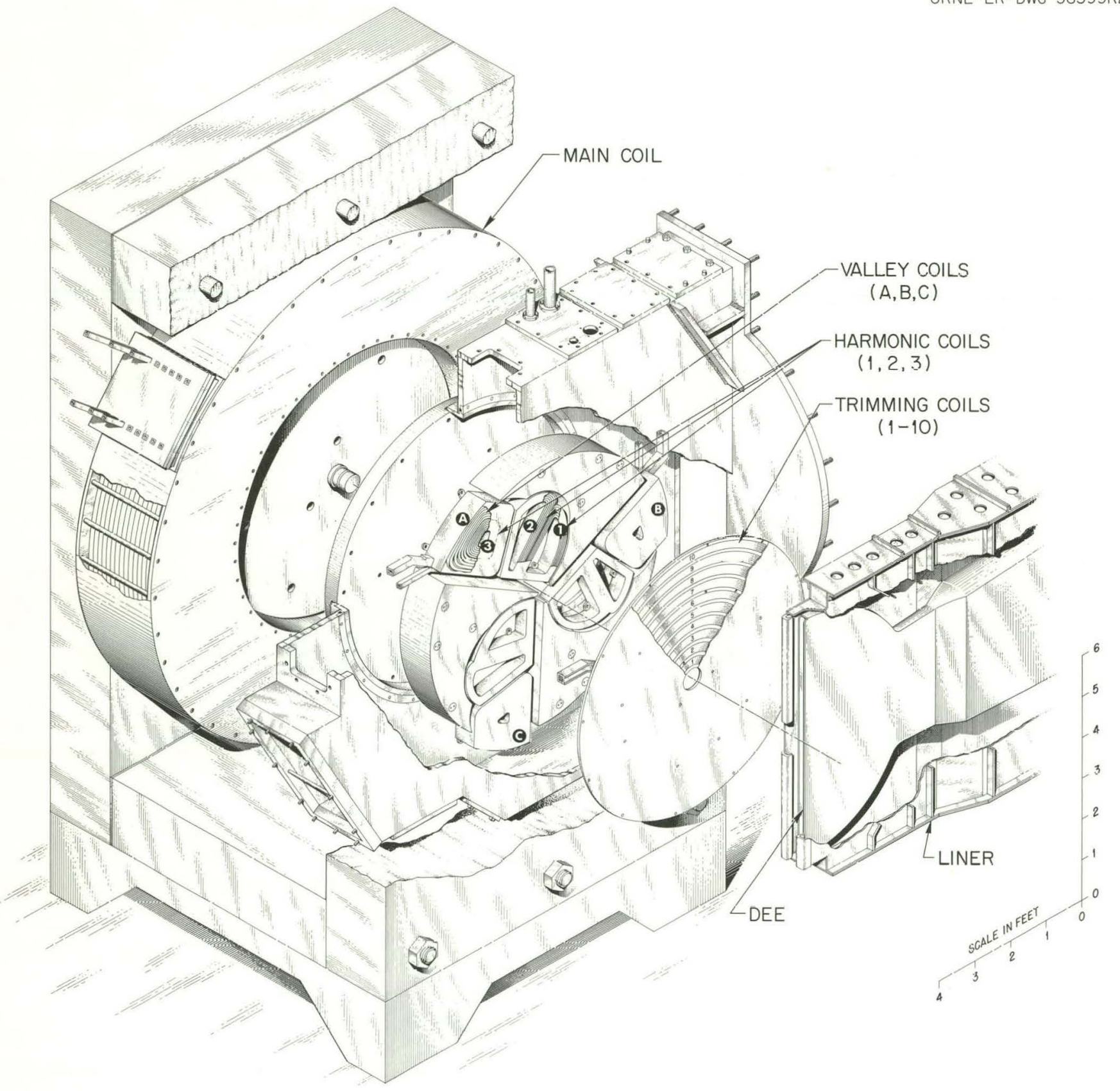

Fig. 6.2. Sketch of ORIC Magnet with One Pole Tip Exploded to Show Associated Coils. 
Table 6.1. Currents in Circular Coils as Optimized by Various Methods, with Test Conditions and Results

\begin{tabular}{|c|c|c|c|c|c|c|}
\hline & \multicolumn{3}{|c|}{ Optimized from } & \multicolumn{2}{|r|}{ Computed } & \multirow{2}{*}{ Limit } \\
\hline & Beam Current & Phase Probe & Phase + Current & Direct & Experimental Adj & \\
\hline \multicolumn{7}{|l|}{ Circular coils, amp } \\
\hline Coil 1 & +356 & +371 & +357 & 0 & 0 & 1080 \\
\hline 2 & -529 & -540 & -560 & -465 & -424 & 900 \\
\hline 3 & 0 & +133 & +104 & +208 & +123 & 801 \\
\hline 4 & +203 & +86 & +136 & 0 & 0 & 700 \\
\hline 5 & +120 & +74 & +68 & +258 & +303 & 675 \\
\hline 6 & +333 & +333 & +334 & +162 & +115 & 550 \\
\hline 7 & +314 & +355 & +347 & +434 & 1436 & 550 \\
\hline 8 & +422 & +439 & +469 & +515 & +506 & 515 \\
\hline 9 & +183 & +229 & +285 & +266 & +280 & 490 \\
\hline 10 & -445 & -415 & -435 & -470 & -461 & 470 \\
\hline \multicolumn{7}{|l|}{ Test conditions } \\
\hline Dee voltage, $\mathrm{kv}$ & 15 & 10 & 10 & 12 & 12 & \\
\hline Radius (max), in. & 33 & 32 & 34 & 24 & 31 & \\
\hline \multicolumn{7}{|l|}{ Beam measured } \\
\hline At $R=32$ in.,$\mu \mathrm{a}$ & 65 & 100 & 180 & & 110 & \\
\hline At $R=20$ in., $\mu \mathrm{a}$ & 305 & 370 & 260 & & 190 & \\
\hline
\end{tabular}

in the region of the circulating beam immediately external to the channel. This channel is in the process of fabrication (see Fig. 6.5). The principle of operation is illustrated in Fig. 6.6. The total excitation in the inner and outer conductors is 200,000 ampere-turns; the field reduction in the beam space is about 6 kilogauss. The circulating beam will travel essentially undisturbed in the notched area of the outer conductor, less than an inch from the deflected beam.

The second section of the magnetic channel moves the deflected beam still farther from the circulating beam. Since more space is available, a more conventional design is being prepared for this section.

\section{ORBIT STUDIES}

The size and location of the first- and secondharmonic magnetic-field bumps were studied. It was shown that asymmetrical excitation of the valley coils could give the beam the radial gain per turn required to enter the electrostatic channel. The shape of the deflector was determined and also the strength and location of the magnetic channel needed to extract the beam.

A complete catalog of coil currents and orbit properties was calculated for protons, alpha particles, and nitrogen ions at nine different energies. This should serve as a useful guide in cyclotron operation. 


\section{UNCLASSIFIED ORNL-LR-DWG 71638}

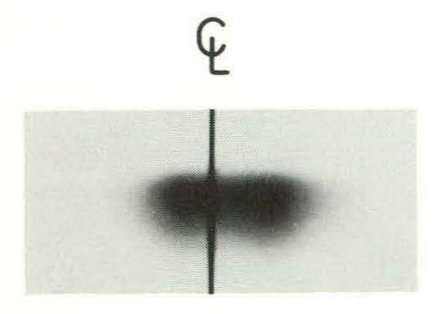

\section{CIRCULATING BEAM AT $R=25$ in.}

$\Psi$

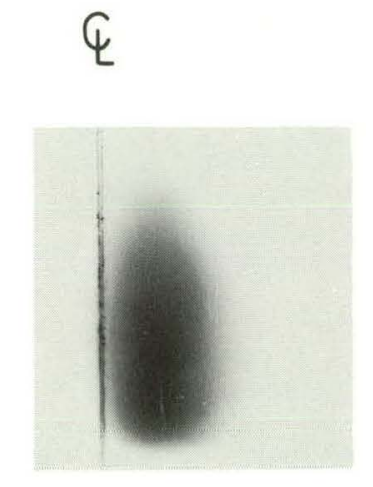

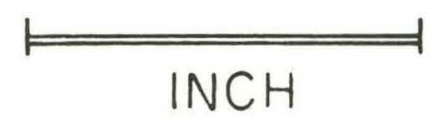

Fig. 6.3. Radioautographs of Beam, Made During Preliminary Testing Program.

\section{RADIO.FREQUENCY SYSTEM}

Early this year the dee and the dee-stem transition were mounted on the end of the 50-in.-diam center conductor of the resonator. This was preceded by a careful alignment procedure to ensure that the dee would come to rest centered in the gap and parallel to the pole-tip faces. While the components were held in position by bolts and shims, the circumferential joint was permanently Heliarc welded with copper.

A temporary if system, using a $4 \mathrm{CX}-5000 \mathrm{~A}$, as the final amplifier, was installed and used for the test operations. The system was controlled from a temporary console in the control room, which allowed motorized tuning of the intermediate amplifier, final amplifier, and resonator trimmers. Phase detectors were used for indicating resonance of amplifiers. An rf voltmeter was used for indicating resonance of the dee system.
The temporary of system was operated at $7.8 \mathrm{Mc}$ with the dee voltage as high as $18 \mathrm{kv}$ peak-toground. This was the maximum voltage that could be obtained within the plate dissipation rating of the 4CX-5000A. During the three months of test operation no attempt was made to increase the frequency; the increased power requirements would have limited the dee voltage to an unreasonably low level. The dee-voltage waveform was observed to be free of modulation and harmonic distortion. This indicates good mechanical stability in the resonator structure.

The initial test program was terminated in July for installation of the permanent power amplifier. This amplifier, including its local control panel, was assembled and placed in position on top of the resonator. The entire system was then raised into position and attached to the support legs of its handling crane, as shown in Fig. 6.1. Since the crane also has $x$ and $y$ translation in a 


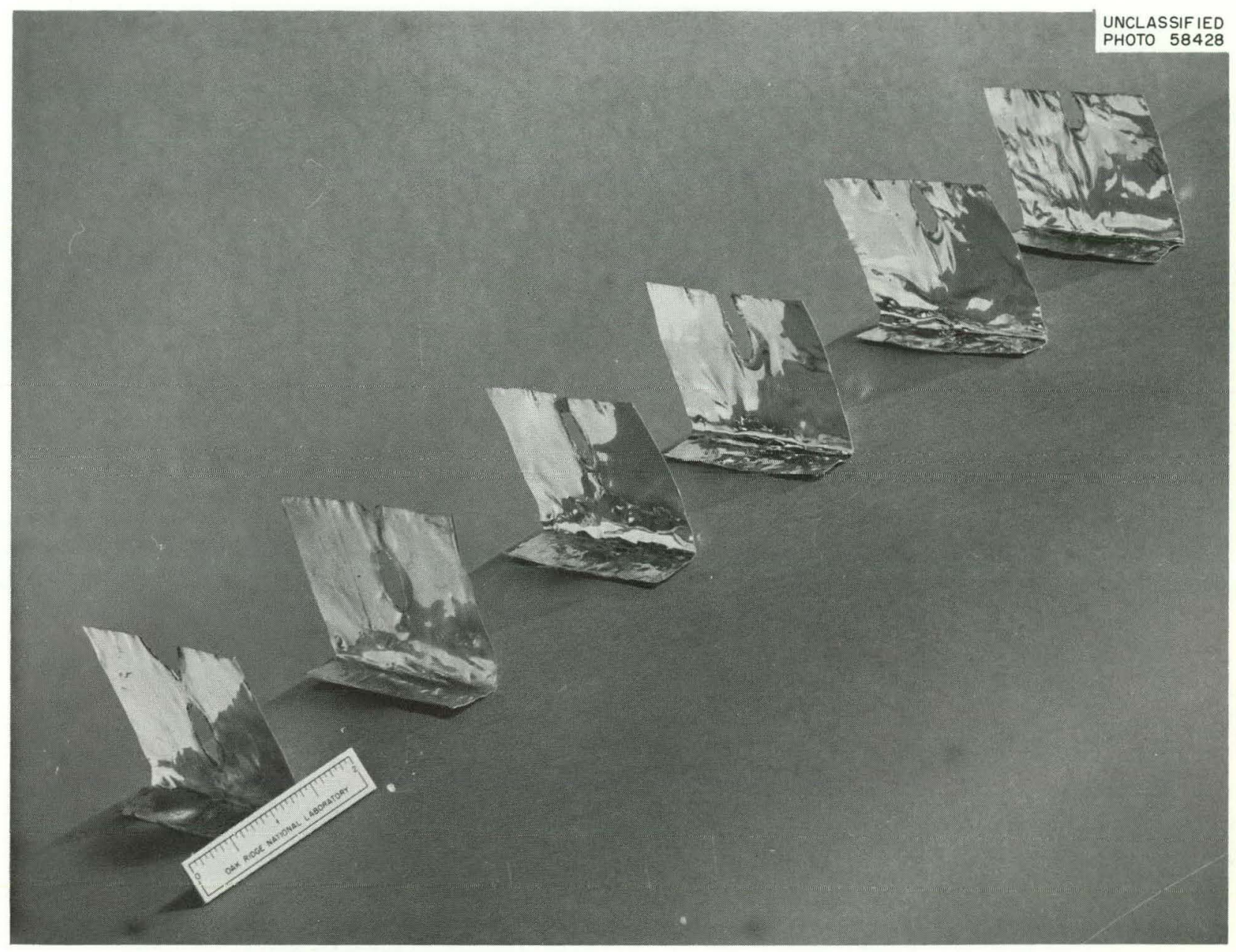

Fig. 6.4. Sequence of Foils Melted by Deflected Beam, Moving Right to Left. 


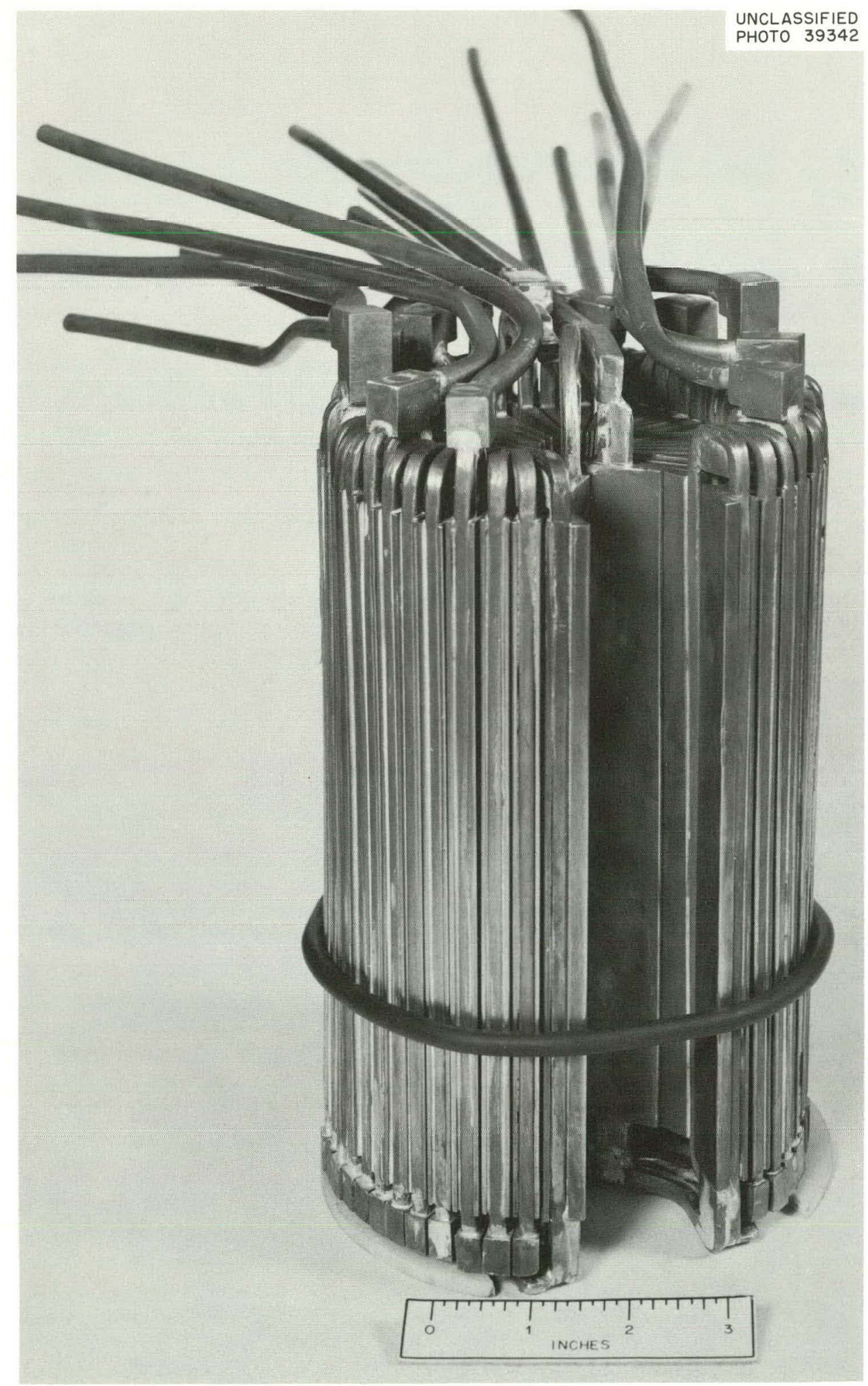

Fig. 6.5. Coaxial Magnetic Channel, Partially Assembled. For design see Fig. 6.6. 


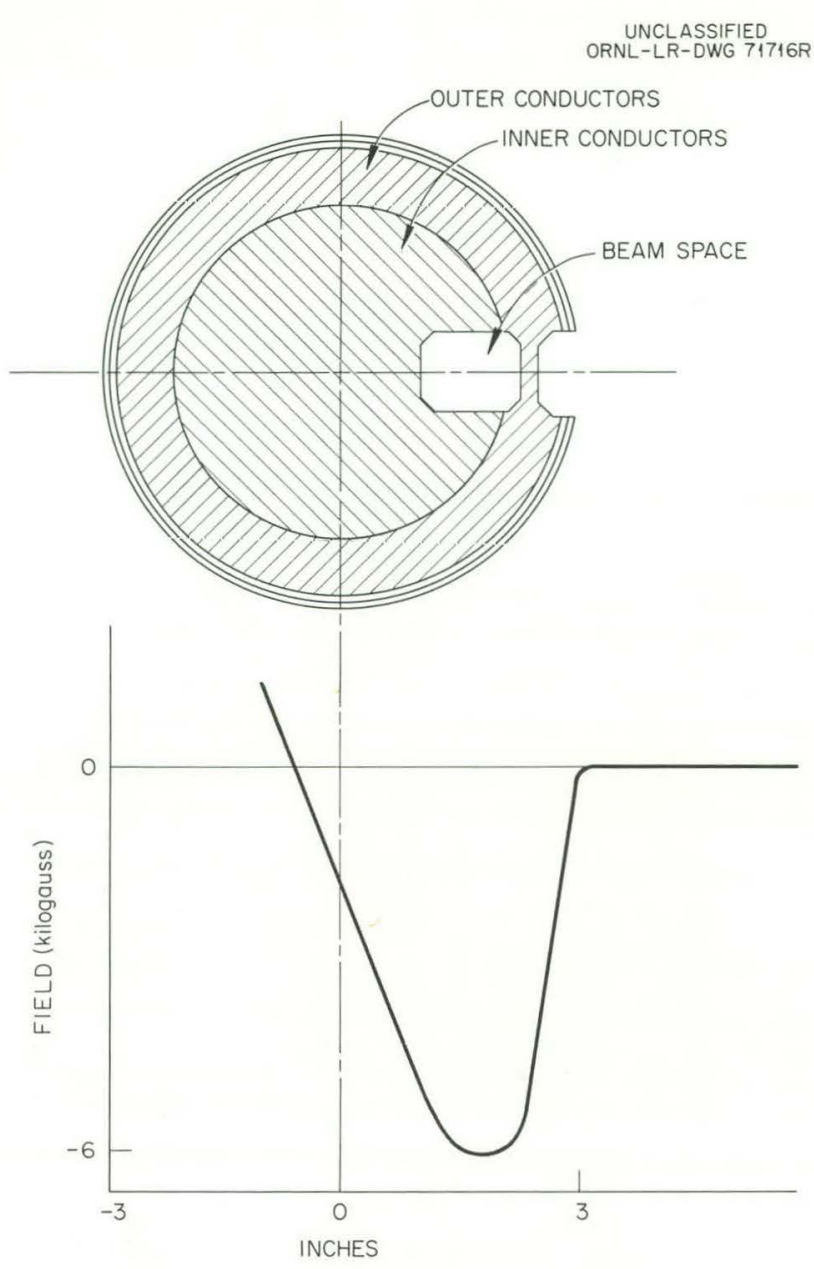

Fig. 6.6. Cross Section of Coaxial Magnet, Showing Sharp Field Cutoff.

horizontal plane, all leads and services were made flexible, or detachable, to provide freedom of motion.

The permanent power amplifier, which uses an RCA 6949, was designed, fabricated, installed, and tested. The grid circuit is a pi network with both $L$ and $C$ variable. Most of the tuning is accomplished by positioning a shorting plane which adjusts the lengths of the $1 / 4$-wave lines extending from the driver-amplifier plate and the power-amplifier grid (see Fig. 6.7). Fixed capacitors are inserted at the driver-amplifier plate and power-amplifier grid for operation between 7 and 11 Mc; they are withdrawn for operation between 11 and $23 \mathrm{Mc}$. Fine tuning is obtained with a variable capacitor on the driver-amplifier plate (see Fig. 6.8).
The tank circuit of the power-amplifier plate has a variable inductance formed by a length of coaxial line with a movable shorting plane, also shown in Fig. 6.7. The line is padded by a gang of four variable capacitors. Only the capacitors are used with the automatic servo for fine tuning, but in selecting a frequency for operation, both inductance and capacitance are varied to maintain a constant $L / C$ ratio.

Water cooling for the plate and grid inductors and forced-air cooling for the external ceramic surface of the power-amplifier tube are provided, in addition to the usual internal water cooling required by the RCA 6949.

Controls for the rf system (Fig. 6.8) were de• signed, installed, and tested. Complete control of the system is possible from either the master console in the control room or from the local controls on the side of the resonator. The resonator and amplifier stages (Fig. 6.9) can be tuned either with manual controls or by automatic servos.

There are four automatic tuning servos (see Fig. 6.10). These include trimmer tuning, poweramplifier plate capacitance tuning, driver-amplifier plate capacitance tuning, and intermediate-amplifier capacitance tuning. Four additional servos, which are presently manually controlled, are the driveramplifier plate inductance servo, the poweramplifier plate inductance servo, the resonatordrive capacitor positioning servo, and the resonatorshorting-plane positioning servo. Controls for automatic operation of the latter four units have been designed and will be installed. It is necessary to manually tune the automatic servos to a region near the resonance condition before they will operate automatically. A preset tuning system was designed for this purpose and will also be installed.

Automatic voltage regulators were designed and installed in both the power-amplifier filament power supply and the dc filament supply used with the driver-amplifier and intermediate-amplifier filaments.

Plug-in circuits are used extensively in the filament regulators and in the tuning servos. Failure of any of these units can be quickly corrected by replacement with a spare circuit board.

The completed rf system has been operated at several frequencies with dee-to-ground voltage as high as $75 \mathrm{kv}$. Higher voltages will be attempted as the checkout program progresses. The system operates quite easily; high rf voltage appears on 


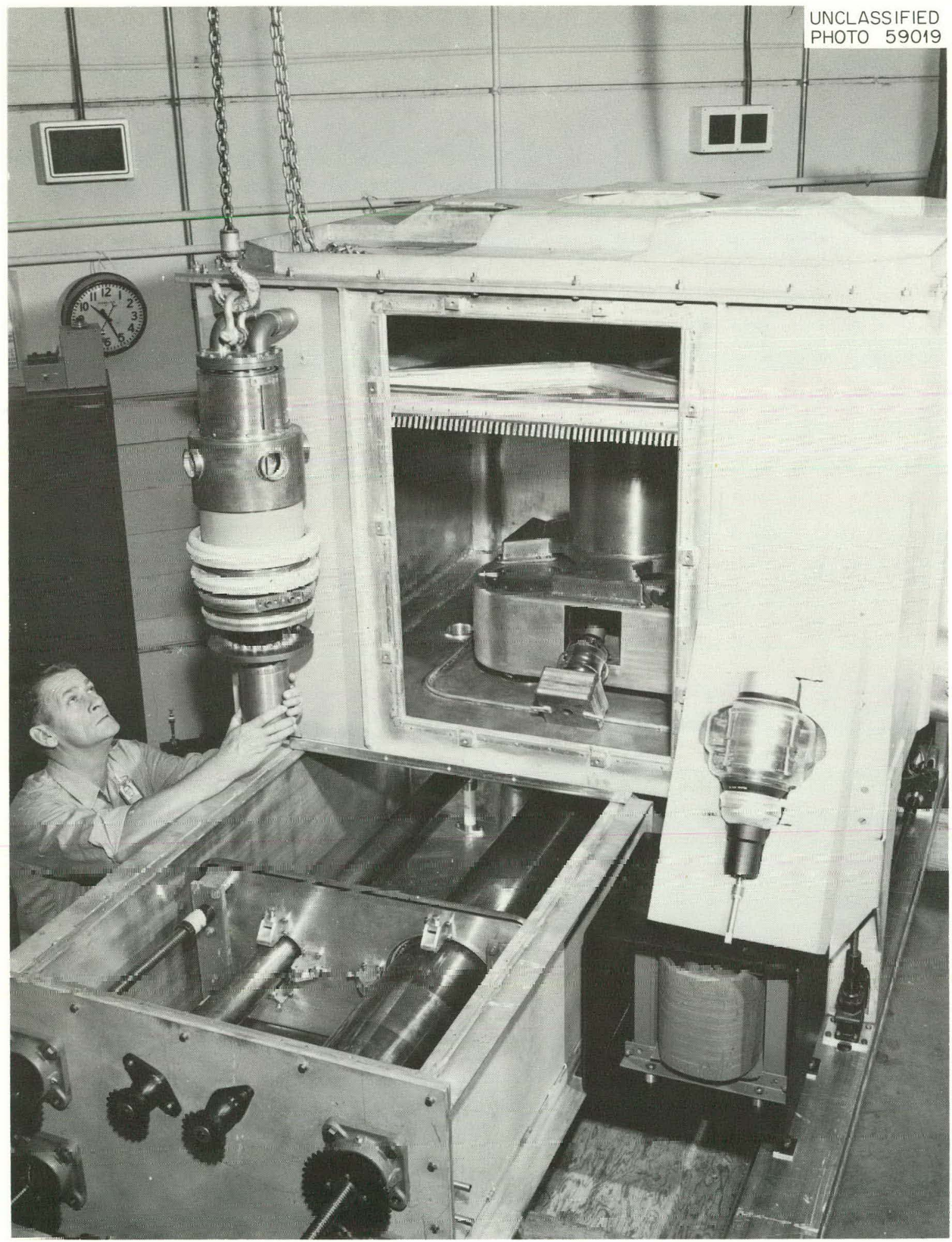

Fig. 6.7. Power Amplifier. The grid tank circuit extends forward from beneath the housing of the plate tank circuit. The RCA 6949, suspended at the left, will be placed inside the central conductor of the variable transmission line of the plate tank circuit. One of the four variable vacuum capacitors for fine tuning of the plate tank circuit is shown on the filament transformer at the right. 


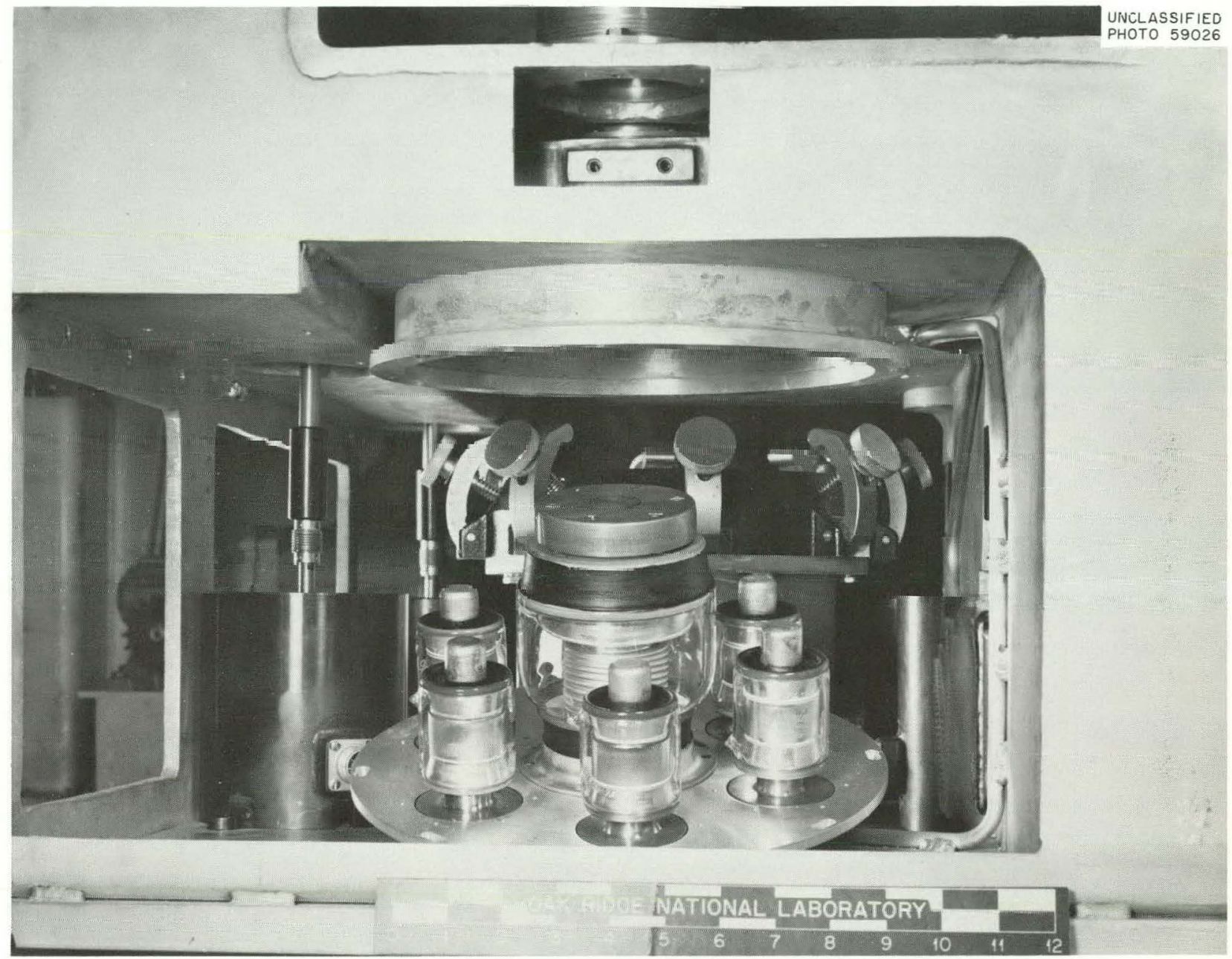

Fig. 6.8. Driver-Amplifier Plate Capacitors. The large central capacitor can be varied for fine tuning the circuit. 


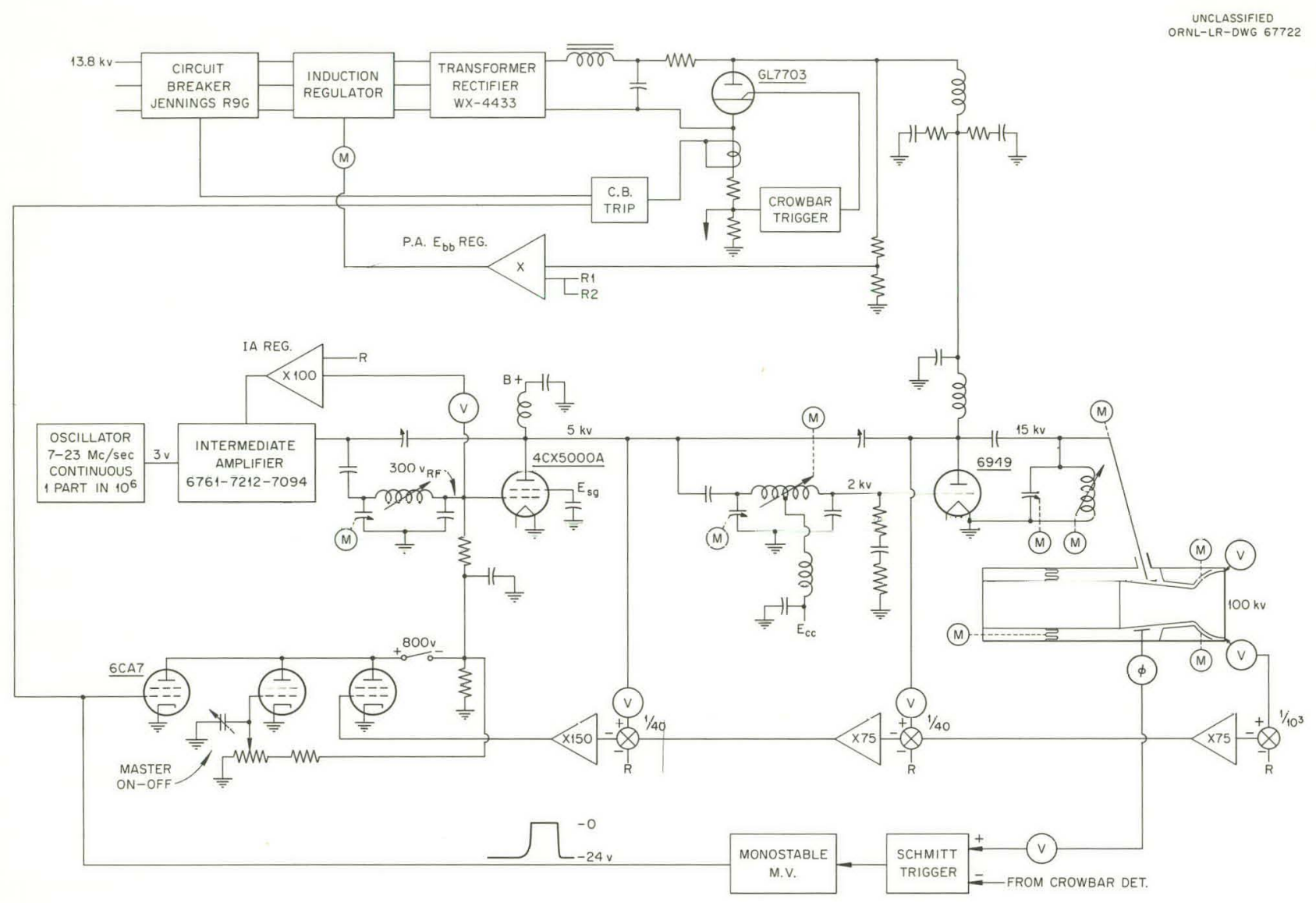

Fig. 6.9. Block Diagram of RF Amplifiers and Regulator Circuits.

the dee as soon as the entire system is in resnnance. No multipactoring or ion-loading problems have been encountered.

A transistorized version of the master signal generator was designed and tested. This unit is expected to be more reliable than the existing vacuum-tube version, but fabrication and installation are optional.

Regulators for the power-amplifier dc plate voltage and the rf dee voltage were designed. Since these units are not vital to operation of the rf system, their fabrication has been temporarily delayed. The power-amplifier dc plate voltage regulator will permit the plate voltage supply to automatically run up to a preset value whenever it is turned on, or reset after firing of the protective crowbar. The dee-voltage regulator would eliminate any modulation present in the rf-voltage waveform on the dee.

\section{ORIC OPERATIONS}

The operations group started 24-hr coverage for five days a week in early March. This coincided with the start of initial testing of the machine. The various stages included: getting operable pressures, establishing adequate cooling to the various components, stabilizing the rf excitation, and accelerating and deflecting a proton beam. The best vacuum obtained at this time was $\sim 1.9 \times$ $10^{-6}$ torr, with a rate of rise of $\sim 0.00045 \mu / \mathrm{sec}$.

The first internal proton beam at full radius of 31.5 in. was obtained on March 18, 1962. The proton energy, $8.0 \pm 0.1 \mathrm{Mev}$, was verified by the well-known stacked-foil technique; the 38-min activity was measured from the reaction $\mathrm{Cu}^{63}(p, n) \mathrm{Zn}^{63}$, for which the threshold is $4.2 \mathrm{Mev}$.

The testing program continued for about three months, during which time numerous internal-beam 


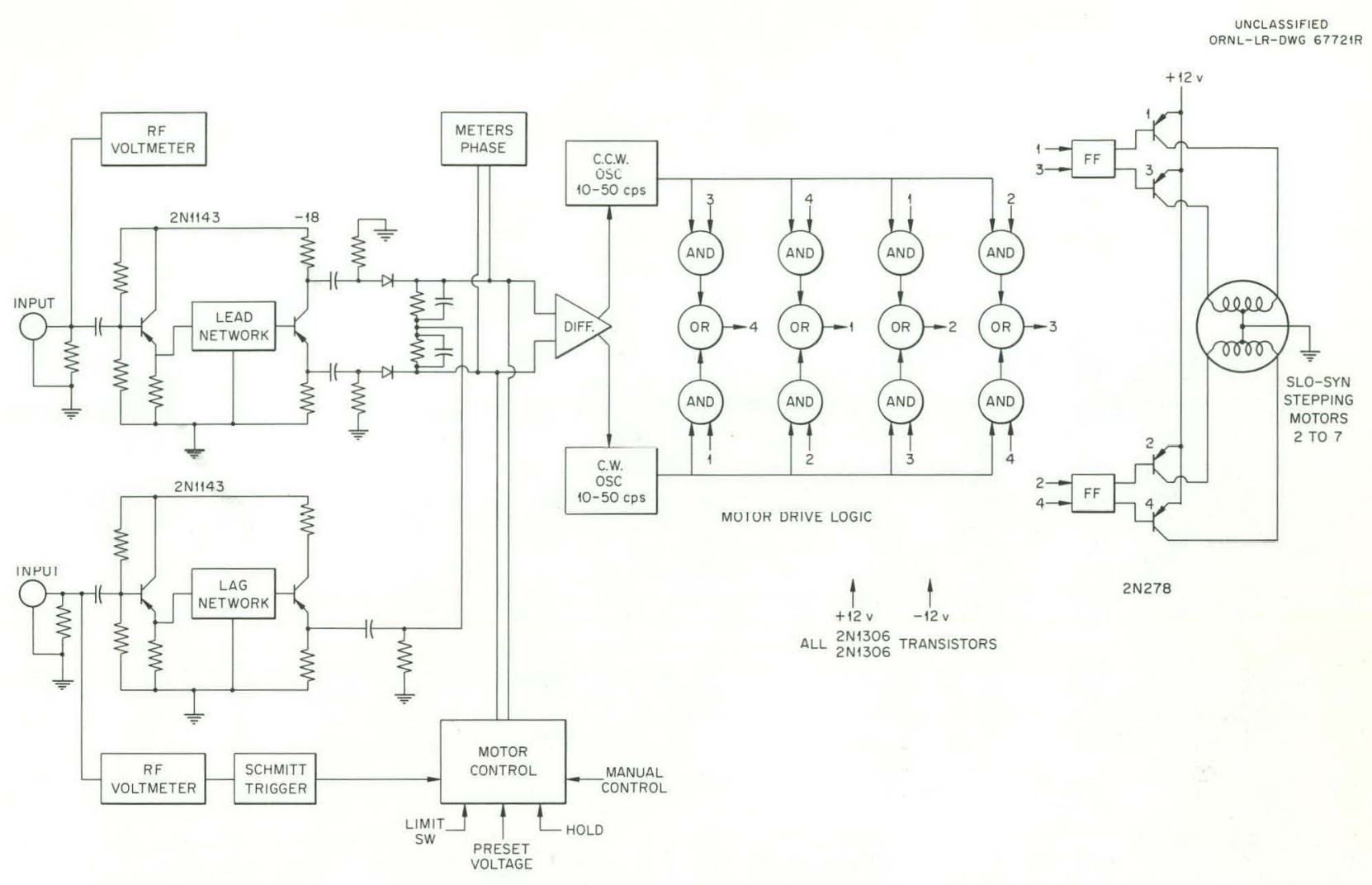

Fig. 6.10. Automatic Tuning Servomechanism for RF System.

studies were made in an effort to determine the machine's operational characteristics.

Following the installation of the permanent power amplifier, operational checkout and testing of the new system were begun in October. By December 20 a stable dee-to-ground potential of $\sim 50 \mathrm{kv}$ was maintained. With this initial success, it is expected that higheenergy beams will become available in the ORIC in January 1963.

\section{ION OPTICS}

The elements of the external ion optics necessary for transmitting the beam into the small experiment room are nearly completed and ready for installation. They include the No. 1 quadrupole, the vertical positioning magnet, and the No. $1\left(45^{\circ}\right)$ beam switching magnet (see Fig. 6.11). Other neccssary quadrupole lenses and the power supplies are now on order.

\section{POWER SUPPLY SYSTEMS}

\section{Magnet Power Supplies}

The 19 power supplies for the pole-face coils which shape the isochronous field were checked out; they were used during cyclotron testing. Failures of certain air-cooled rectifier diodes, due to overheating, prompted mounting these diodes on water-cooled heat sinks. Since this has worked quite well, water cooling will be installed on all units as soon as possible.

\section{Ion-Source Supplies}

Power supplies for the ion-source filament and arc were installed and operated. These units were obtained as surplus from electromagnetic separation units $(\mathrm{Y}-12)$ and were modified for operation in the cyclotron. The arc current is controlled by varying the filament power. 


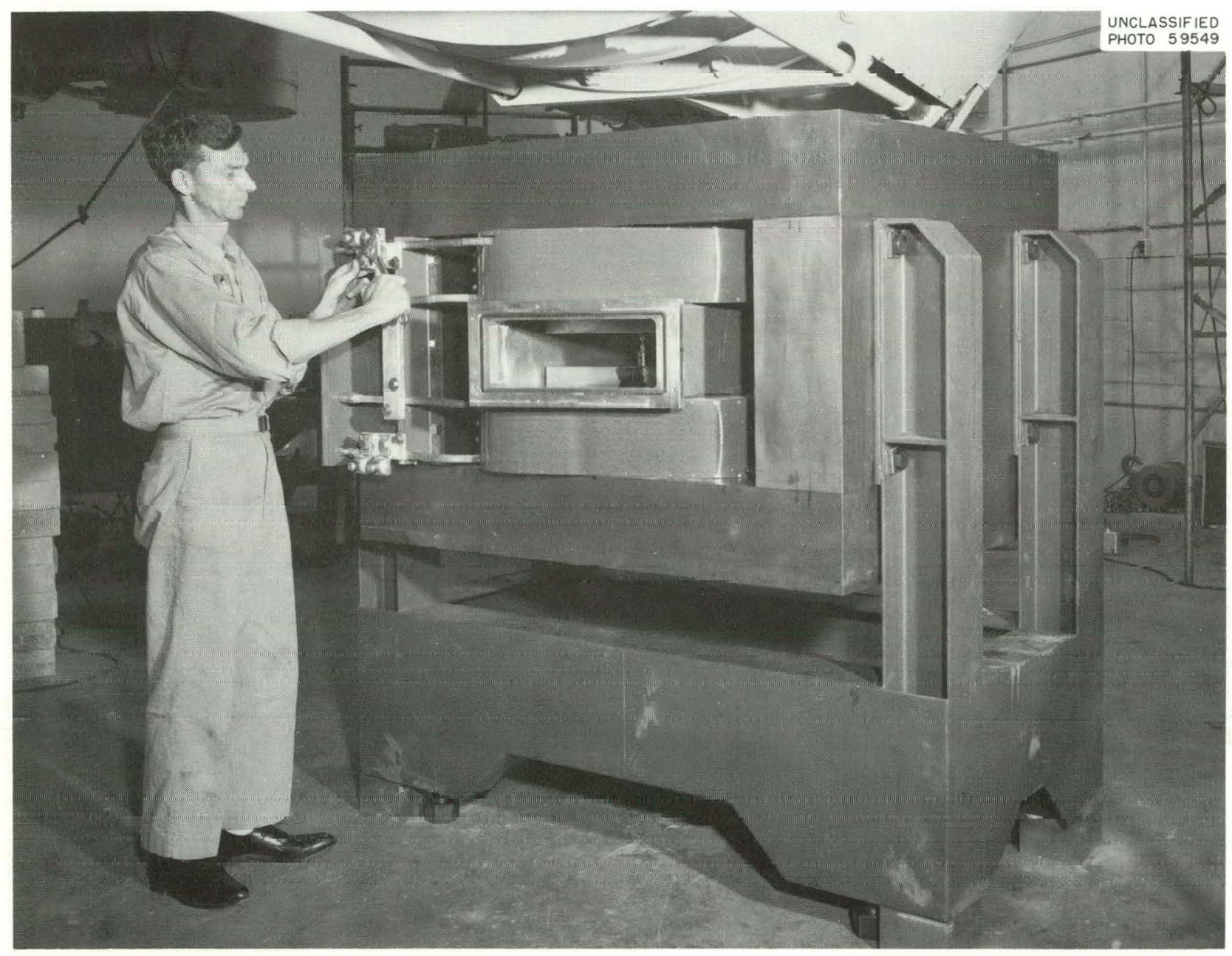

Fig. 6.11. The No. $1\left(45^{\circ}\right)$ Beam-Switching Magnet. 


\section{Deflector Power Supply}

A $120-\mathrm{kv}, 30-\mathrm{ma}$, power supply for the electrostatic deflector was purchased and installed. It was found that incorporation of a 1 -megohm series resistor at the end of the cable nearest the deflector greatly improves the ability of the deflector to hold voltage. Up to $80 \mathrm{kv}$ has been applied to the deflector.

\section{CYCLOTRON CONTROL SYSTEM}

With the installation of the rf-system controls in the control room, the central cyclotron control station at the console is nearly complete. Controls for external ion handling are not yet installed and operating, but these will be completed as the ion-optics systems are installed. Vacuum controls and beam-pipe valving, beam-handling magnets and power supplies, and beam plugs and stops are being built or purchased.

\section{PHASE.PROBE SYSTEM}

A system for measuring the phase of the circulating ion beam with respect to the applied if voltage was developed and tested. It measures the phase of the bunched ions in the beam without in any way disrupting the beam. The system, shown in Fig. 6.12, consists of a shielded pickup plate which detects the electromagnetic wave created by the motion of ions in the beam. The signal developed on this line is fed to a sampling oscilloscope which is triggered by an rf signal sampled from the dee. Oscilloscope measurement of the phase is made by observing the relative displacement of the if waveform and the beaminduced waveform (see Fig. 6.13).

The system proved very valuable for adjusting the currents in the various coils in the magnet to achieve isochronism. Beam phase can be observed and measured at any radius from 3 to 34 in. A system of fixed probes will be installed at various radii in the machine to aid in maintaining isochronism during operation.

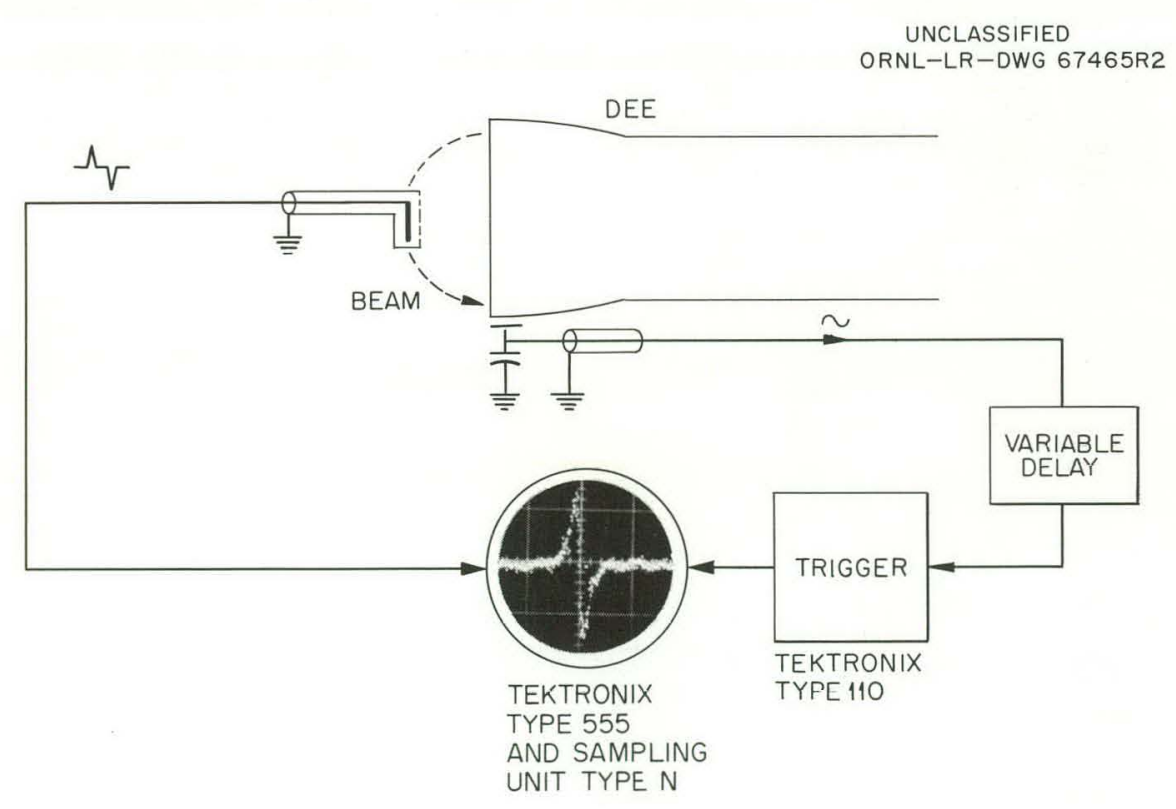

Fig. 6.12. Scheme Used for Beam-Phase Measurements. 


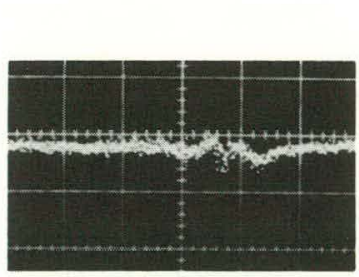

31.5 in.

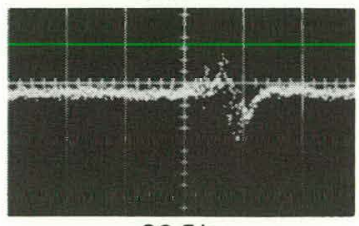

29.5 in.

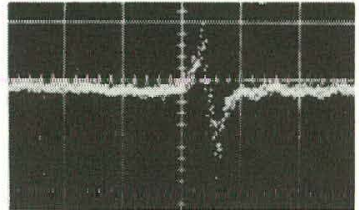

27.5 in,

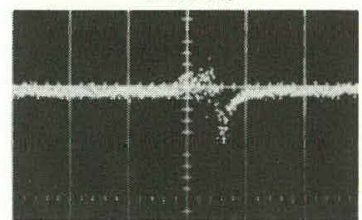

25.5 in.

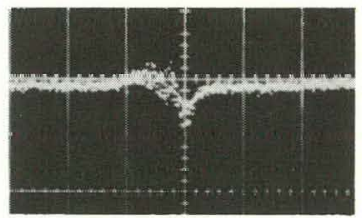

23.5 in.

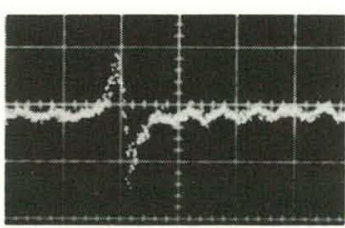

$21.5 \mathrm{in}$.

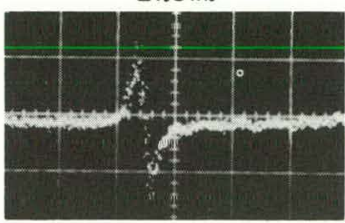

19.5 in.

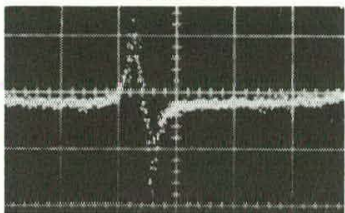

$17.5 \mathrm{in}$.

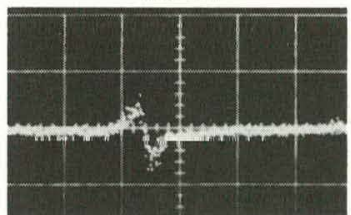

15.5 in.

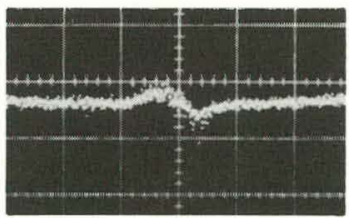

$13.5 \mathrm{in.}$

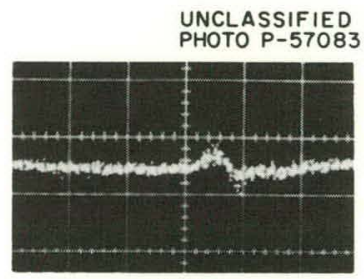

11.5 in.

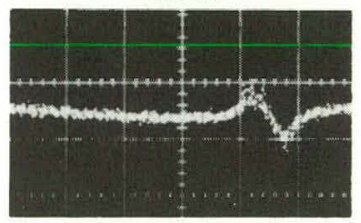

9.5 in.

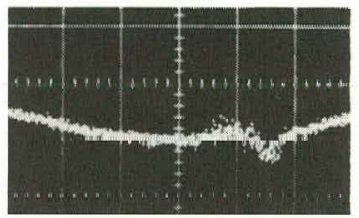

$7.5 \mathrm{in}$.

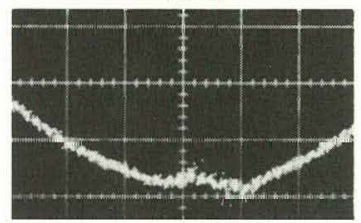

5.5 in.

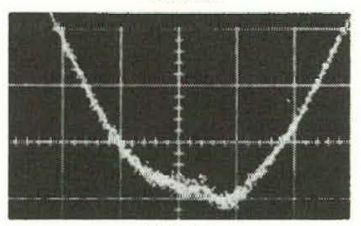

3.5 in.

Fig. 6.13. Beam-Phase Waveforms at Various Radii. The sweep is 10 nanoseconds/cm and the vertical sensitivity $10 \mathrm{mv} / \mathrm{cm}$. 


\section{Superconducting-Magnet Development}
R. W. Boom
C. J. Bergeron ${ }^{1}$
L. D. Roberts ${ }^{2}$
J. A. Martin
R. J. Deck
S. T. Sekula ${ }^{3}$

Three of the basic problems connected with the design of large niobium-zirconium superconducting magnets were investigated during the year. These are "current degradation," protection and safety, and transitions to the normal state.

Current degradation refers to the puzzling anomaly of current capacity. It was found early at many laboratories, including ORNL, that most small-bore magnets made of 0.010 -in. $\mathrm{Nb}-25 \% \mathrm{Zr}$ wire carry only about 20 amp for all fields below critically high fields, even though short-wire tests seemingly indicate much higher currents should be expected. Later it was found that $20 \mathrm{amp}$ is not an invariant, but that it decreases as the diameter of the magnet is made larger, and that it also depends on other factors. Techniques mentioned below show that the 20 -amp capacity can be increased.

Protection and safety refers to the transition of a magnet from the superconducting state. It was found that transitions initiate at one point, propagate at thermal velocities, and heat the wires by Joule heating. As a result the field decays with a time constant $L / R$. This produces voltages large enough to damage insulation; it also results in overheating of the conductor, commonly with melting at the first point of transition.

Basic to the understanding of superconducting magnets and to the solution of associated problems are detailed measurements of all quantities, electrical and thermal, which describe the transition. Interpretation of our measurements is presented below.

\footnotetext{
${ }^{1}$ Consultant, Louisiana State University, New Orleans.

${ }^{2}$ Physics Division.

${ }^{3}$ Solid State Division.
}

\section{MAGNET TESTS}

A series of $1 / 2$-in.-ID test magnets potted in epoxy resin produce 15 to 30 kilogauss and carry 29 to $30 \mathrm{amp}$, an increase of $50 \%$ over the usual 20 -amp capacity of unpotted magnets. An obvious advantage of epoxy potting is that the epoxy provides solid thermal contact to a massive medium.

The ORNL magnets are usually bifilar wound with $20 \%$ of the volume nylon-insulated 10 -mil Nb$\mathrm{Zr}$ wire, 20\% Formvar-insulated 10-mil copper wire, and $60 \%$ epoxy. Some magnets are wound on copper forms and some on brass forms, but most are on epoxy forms. The copper secondaries supply protection during field collapse by absorbing a large fraction of the field energy, $1 / 2 L I_{0}^{2}$, and by slowing down the rate of decay, thus reducing induced voltages.

For a magnet study, the copper secondary, in 1:1 coupling with the primary, supplies a very convenient method for measuring the $\mathrm{Nb}-\mathrm{Zr}$ resistance as it develops. If the $\mathrm{Nb}-\mathrm{Zr}$ primary and the copper secondary are both separately shorted, but disconnected from other circuitry and from each other, then

$$
I_{p} R_{p}=I_{s} R_{s} .
$$

Since $I_{p}, I_{s}$, and $R_{s}$ are easily measured as functions of time, $R_{p}$ can be known as a function of time. It is found that, in the transition from the superconducting to the normal state, the resistance of the primary increases linearly with time; that is,

$$
R_{p} \cong \alpha t,
$$

where $\alpha$ ranges from $10^{3}$ to $10^{4} \mathrm{ohms} / \mathrm{sec}$ for this type of construction. 
Oscilloscope traces of $I_{p}$ and $I_{s}$ during a field collapse are shown in Fig. 7.1; the computed $R_{p}$ and energy dissipation are given in Fig. 7.2. It is seen that only $5 \%$ of the energy is dissipated in the $\mathrm{Nb}-\mathrm{Zr}$ primary. It can be shown that an approximate expression for $I_{p}$ during the field collapse is

$$
I_{p}=I_{0} \frac{e^{-t R_{s} / L}}{1+\alpha t / R_{s}}, \quad \text { for } R_{p}=\alpha t .
$$

Most of the energy stored in the $\mathrm{Nb}-\mathrm{Zr}$ windings is dissipated promptly; if $t \leqq 10 R_{S} / \alpha$, the energy dissipated in the $\mathrm{Nb}-\mathrm{Zr}$ is

$$
E(\mathrm{Nb}-\mathrm{Zr})=1.5 I_{0}^{2} R_{s}^{2} / \alpha \text { joules } .
$$

For our magnets, $R_{s} \cong 1 \mathrm{ohm}, \alpha \cong 10^{3} \mathrm{ohms} / \mathrm{sec}$, and $I_{0} \cong 30 \mathrm{amp}$; so $E(\mathrm{Nb}-\mathrm{Zr}) \cong 1.5$ joules. It is best to distribute this energy as widely as possible; thus the copper should be of high purity (low $R_{s}$ ), and the construction should be such that $a$ is as large as possible.

At the point of initial transition the energy dissipated per unit length is

$$
E / \mathrm{cm}=0.06\left(R_{s} / \alpha\right) I_{0}^{2} \text { joules } .
$$

The energy required to increase the temperature of the $\mathrm{Nb}-25 \% \mathrm{Zr}$ (initially at $4.2^{\circ} \mathrm{K}$ ) by $300^{\circ} \mathrm{K}$ is calculated $^{4}$ to be 55 joules/g. Thus, to ensure that no point in the sample goes above room temperature, the energy dissipated should not exceed 0.2 joule $/ \mathrm{cm}$.

The voltage across the normal part of the $\mathrm{Nb}-\mathrm{Zr}$ is

$$
V \cong I_{0} R_{s} \frac{e^{-t R_{s} / L}}{1+R_{s} / \omega t} .
$$

\footnotetext{
${ }^{4} \mathrm{P}$. Smith, Harwell, private communication.
}

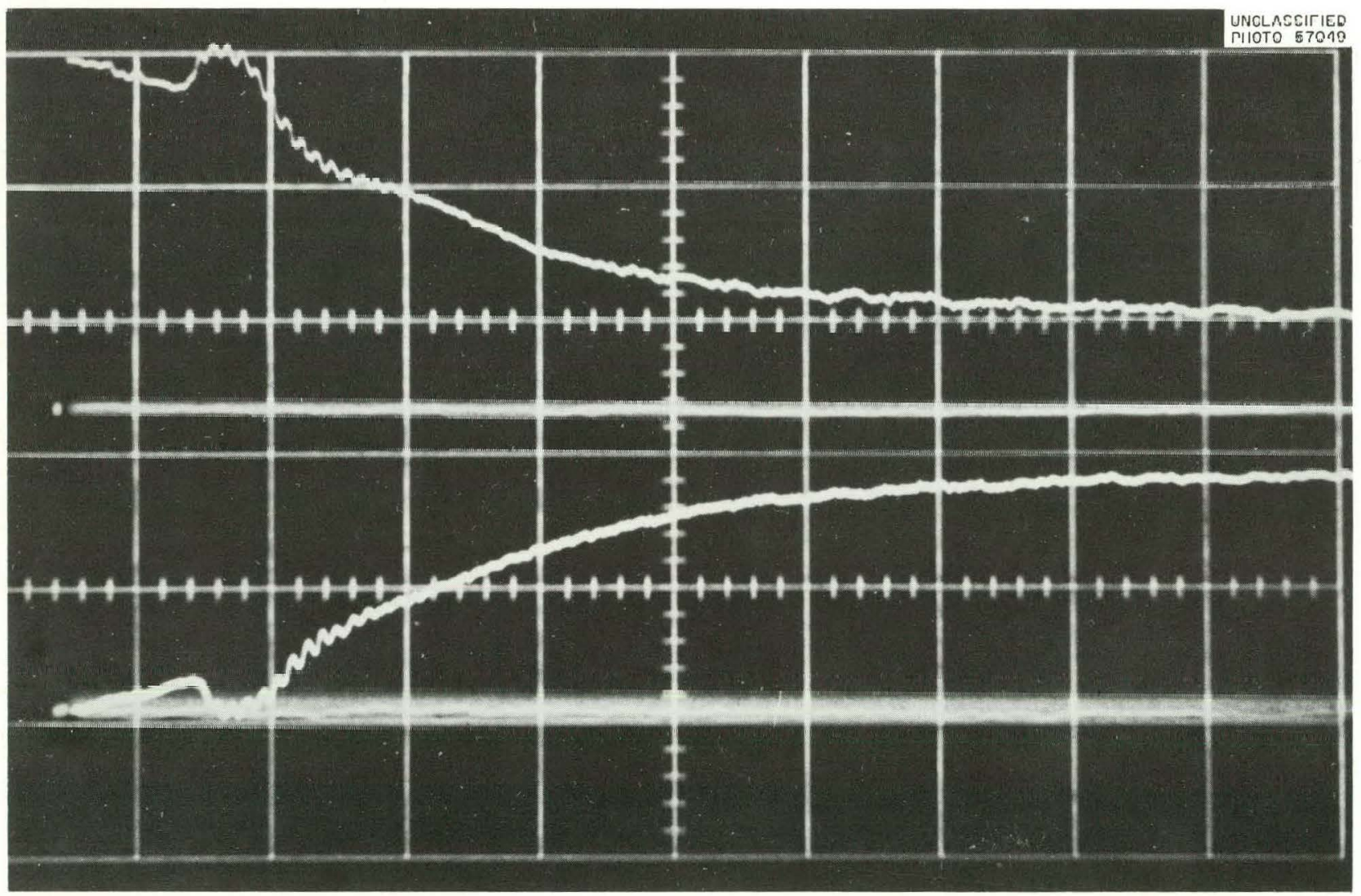

Fig. 7.1. Field Collapse in Magnet B with the Secondary Closed. Time base is $0.050 \mathrm{sec} / \mathrm{cm}$. Upper trace is primary $(\mathrm{Nb}-\mathrm{Zr})$ current; lower trace is secondary current, at $10 \mathrm{amp} / \mathrm{cm}$. 


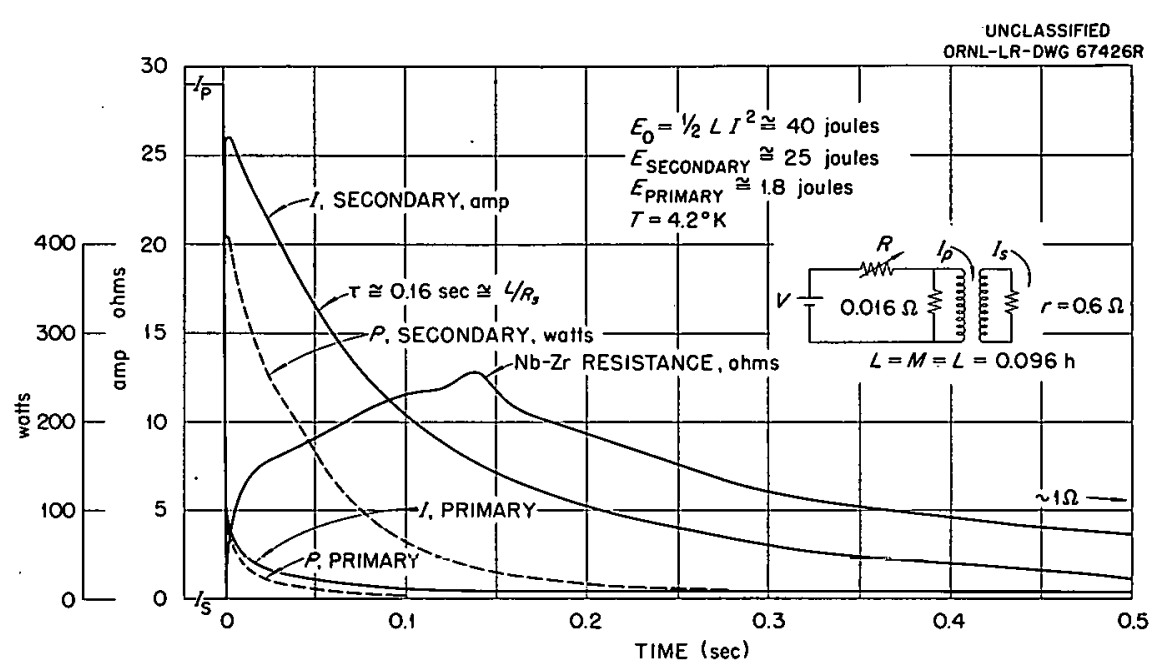

Fig. 7.2 Data from Field Collapse in Magnet B with Secondary Closed. Primary and secondary currents, power, and resistance as computed from Fig. 7.1 .

Table 7.1. Test Magnets (0.010-in. $\mathrm{Nb}-25$ at. \% $\left.\mathrm{Z}_{r}\right)$

lemperature, $4.2^{\circ} \mathrm{K}$

\begin{tabular}{|c|c|c|c|c|c|c|c|}
\hline & \multicolumn{7}{|c|}{ Magnet } \\
\hline & \multirow{2}{*}{$\begin{array}{c}\text { A, } \\
\text { Open } \\
\text { Winding }\end{array}$} & \multicolumn{2}{|c|}{$\begin{array}{c}\text { B } \\
\text { Secondary, } \\
\text { Epoxy }\end{array}$} & \multicolumn{2}{|c|}{$\begin{array}{c}\text { C, } \\
\text { Secondary, } \\
\text { No Epoxy }\end{array}$} & \multicolumn{2}{|c|}{$\begin{array}{c}\text { D, } \\
\text { Secondary, } \\
\text { Epoxy }\end{array}$} \\
\hline & & Closed & Open & Closed & Open & Closed & Open \\
\hline Current, $\max (\mathrm{amp})$ & 18 & 34 & 18 & 24 & 24 & 32 & 16 \\
\hline Field, $\max$ (kilogauss) & 29 & 20 & 10.6 & 17.5 & 17.5 & 28 & 14 \\
\hline Wire length $(f t)$ & 1800 & \multicolumn{2}{|c|}{1000} & \multicolumn{2}{|c|}{850} & \multicolumn{2}{|c|}{1700} \\
\hline $\begin{array}{l}\text { Winding density } \\
\text { (turns } / \mathrm{cm}^{2} \text { ) }\end{array}$ & 950 & \multicolumn{2}{|c|}{300} & \multicolumn{2}{|c|}{469} & \multicolumn{2}{|c|}{333} \\
\hline Induct ance (henries) & 0.37 & \multicolumn{2}{|c|}{0.13} & \multicolumn{2}{|c|}{0.098} & \multicolumn{2}{|c|}{0.25} \\
\hline \multicolumn{8}{|l|}{ Size (in.) } \\
\hline ID & $1 / 2$ & \multicolumn{2}{|c|}{$1 / 2$} & \multicolumn{2}{|c|}{$1 / 2$} & \multicolumn{2}{|c|}{$1 / 2$} \\
\hline OD & 2 & \multicolumn{2}{|c|}{$2 \frac{1}{2}$} & \multicolumn{2}{|c|}{2} & \multicolumn{2}{|c|}{$2 \frac{1}{2}$} \\
\hline Length & $1 \frac{1}{4}$ & \multicolumn{2}{|c|}{$1 \frac{1}{2}$} & \multicolumn{2}{|c|}{$1 \frac{1}{4}$} & \multicolumn{2}{|c|}{2} \\
\hline
\end{tabular}

For low voltages, $R_{s}$ should be small and the time constant $L / R_{s}$ should be long.

The magnets built and tested are shown in Table 7.1. Magnet A was wound without epoxy or secondary and carried the expected 18 amp. Magnet $C$, with a secondary but without epoxy, carried slightly more current but not as much as magnet $B$, which was potted in epoxy. Magnet $D$, also potted in epoxy, was destroyed during the first tests with the secondary open, confirming the above-mentioned safety limits. Also, another magnet, with 2-in. ID, $1 \frac{1}{2}$-in. length, and $3 \frac{3}{4}$-in. OD, was wound on an epoxy form with a copper secondary and potted in epoxy. It carries a maximum of 38 amp and produces 14 kilogauss at the center and 18 kilogauss maximum on any winding. The measurements show 
that $\alpha \cong 10^{3} \mathrm{ohms} / \mathrm{sec}$; it should be similar to other $a$ 's because the thermal conditions are similar.

\section{MATERIALS EVALUATION}

Various superconducting alloys were evaluated in the ORNL High-Field Solenoid, which provides

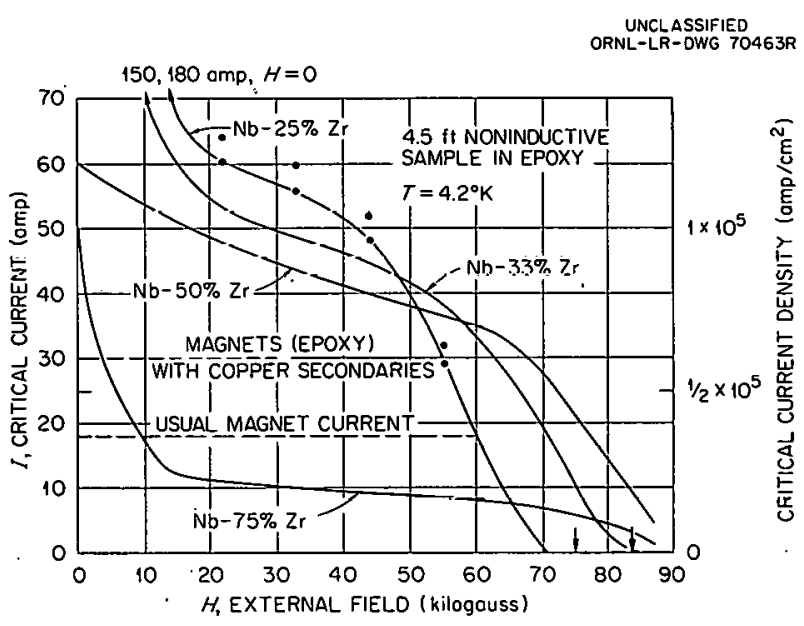

Fig. 7.3. Transverse Field Tests of Commercially Available 10-Mil Nb-Zr Wire of Varying Compositions.

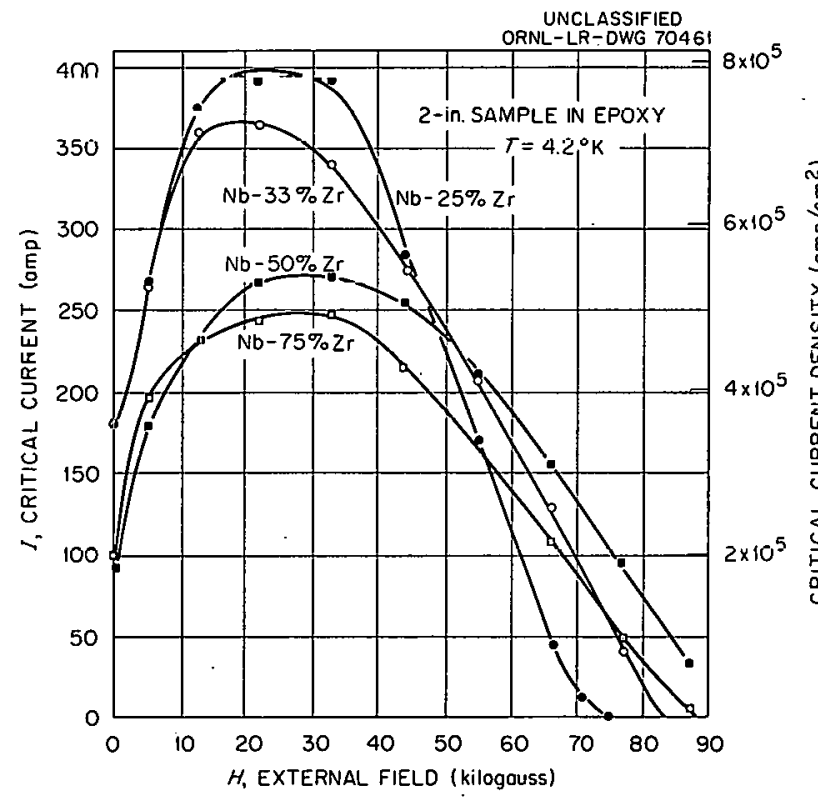

Fig. 7.4. Longitudinal Field Tests of Commercial 10-Mil Nb-Zr Wire of Varying Compositions. fields up to 100 kilogauss. Results for commercial 10-mil Nb-Zr wire are shown in Fig. 7.3. The $\mathrm{Nb}-25 \% \mathrm{Zr}$ is the standard Wah Chang product with over $99.9 \%$ reduction in area during the initial drawing and swaging processes. The $\mathrm{Nb}-33 \% \mathrm{Zr}$ is a selected best sample which was heat-treated at $790^{\circ} \mathrm{C}$ for $15 \mathrm{~min}$ before drawing. The $\mathrm{Nb}-50 \%$ $\mathrm{Zr}$ is a selected best sample heat-treated the same way. The only available sample of $\mathrm{Nb}-75 \% \mathrm{Zr}$ was not heat-treated.

Short-wire longitudinal tests were made for NbZr (Fig. 7.4), Ti-Ta (Fig. 7.5), and Mo-Re (Fig. 7.6). All tests showed large peaks in the current capacity of the wire at intermediate field intensities. It is speculated that this behavior is due to helical (possibly force-free) current flow. A coroid was constructed to confirm these results. One layer of 10-mil wire was wound on a 4-in.-diam, $1 / 2$-in. cross-section form so that each wire was at $45^{\circ}$ to a radius vector. This is the prescription for making a force-free toroid in which the current sheet and the field are everywhere parallel. A maximum current of $120 \mathrm{amp}$ was carried in a local self field of about 4 kilogauss. Inspection of Fig. 7.4 shows that the current in the parallel field should have been about 240 amp. This high current tends to confirm short-wire tests; construction difficulties which prevent perfect $45^{\circ}$ sheet flow may account for the difference noted.

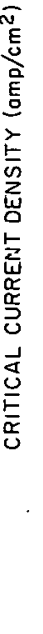

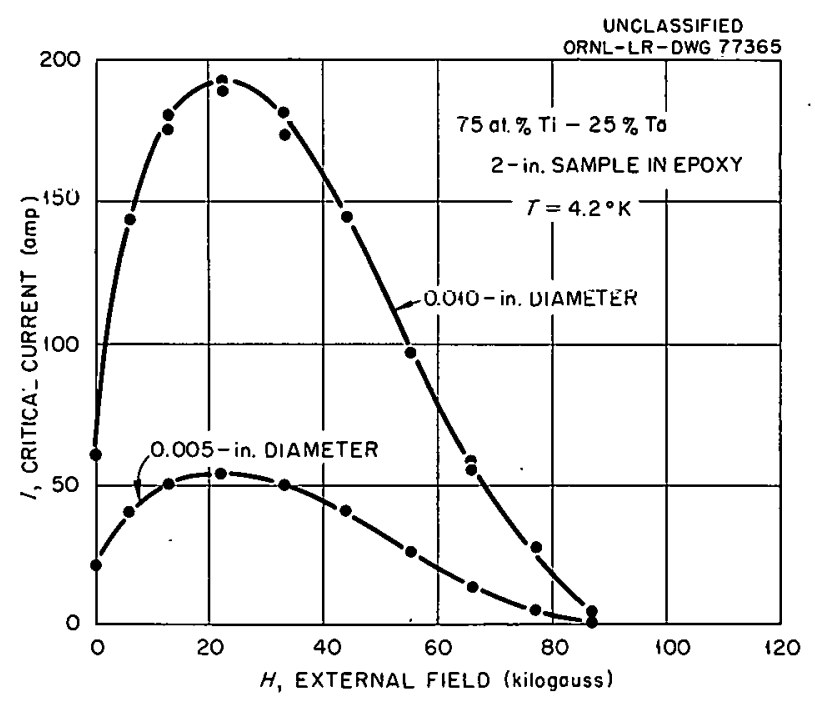

Fig. 7.5. Tests of Ti-Ta Wire in Longitudinal Field. 
Tests with small four- to eight-layer magnets in external fields confirm earlier results; that is, the peaks in the $I-H$ curve are similar to those found for longitudinal samples. Typically, a small $\frac{1}{2}$-in.ID, 1-in.-long, eight-layer magnet of $\mathrm{Nb}-25 \% \mathrm{Zr}$ 10-mil-diam wire will carry $20 \mathrm{amp}$ in its self field, $40 \mathrm{amp}$ in an external field of 20 kilogauss, and approximately the same currents predicted by transverse short-wire tests in external fields higher than 20 kilogauss. This peaking indicates that in large $\mathrm{Nb}-\mathrm{Zr}$ magnets, the region in which a superconducting-to-normal transition initially occurs could be in either a high- or a low-field region of the windings. Possibilities of optimizing the current capacities of superconducting magnets will be evaluated with a multisection magnet now under construction.

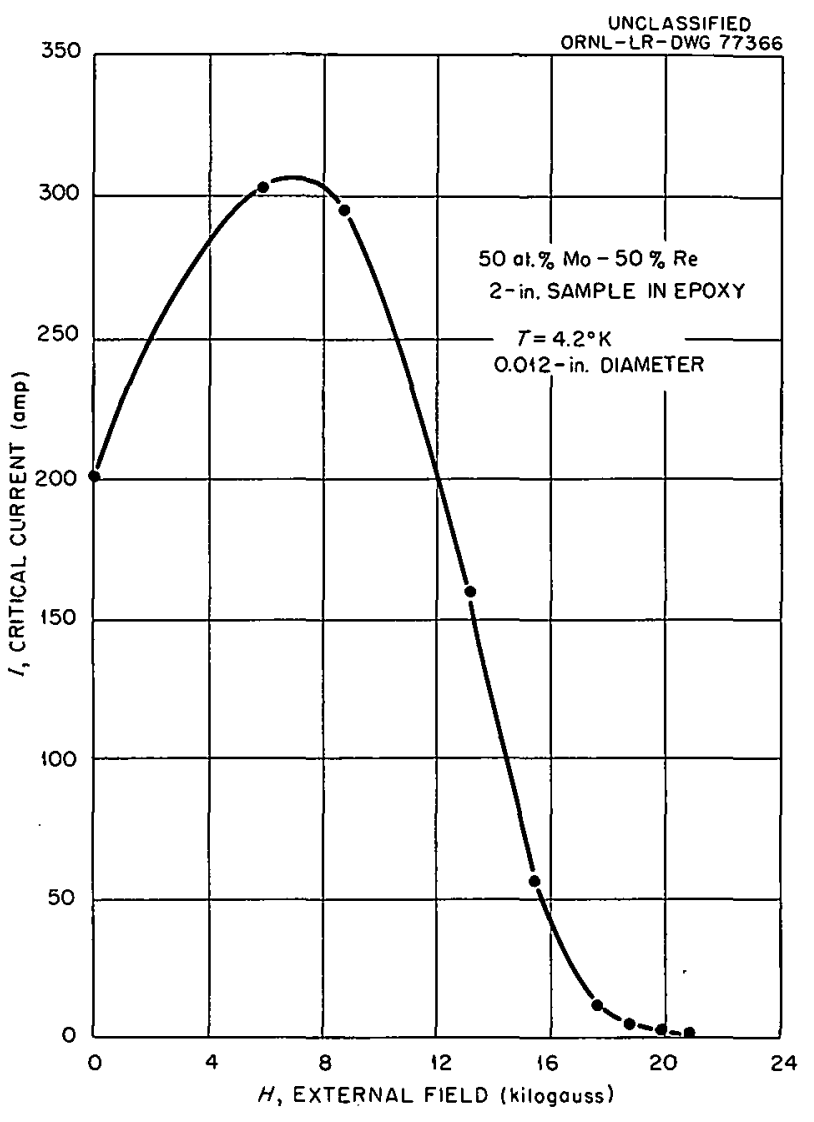

Fig. 7.6. Test of a Sample of Mo-Re Wire in Longitudinal Field. 


\section{Papers Presented at Scientific and Technical Meetings}

American Pbysical Society, New York, January 24-27, 1962

J. B. Ball, C. D. Goodman, and C. B. Fulmer, "A Comparison of Cross Sections for $(p, d)$, $(p, t)$, and $(p, \alpha)$ to the Same Final States."

C. B. Fulmer, J. B. Ball, and C. D. Goodman, " $(p, t)$ Reactions in Zirconium Isntopes."

C. D. Goodman, C. B. Fulmer, and J. B. Ball, " $(p, d)$ Reactions in Zirconium Isotopes."

J. W. Wachter, "Differential Cross Sections of the $\mathrm{Li}^{6}\left(\mathrm{~N}^{14}, \alpha\right) \mathrm{O}^{16}$ and $\mathrm{Li}^{7}\left(\mathrm{~N}^{14}, \alpha\right) \mathrm{O}^{17}$ Reactions."

Southeastern Section of the American Physical Society, Tallabassee, Florida, April 5-7, 1962

J. B. Ball, C. D. Goodman, and C. B. Fulmer, "The Nuclear Deformation of $\mathrm{Mn}^{55}$ Inferred from the $\mathrm{Mn}^{55}(p, d)$ Reaction with 22-Mev Protons."

C. J. Bergeron, L. D. Koberts, and R. W. Boom, "Superconducting Tests of NbZr in a High-Field Magnet."

R. W. Boom and L. D. Roberts, "Transformer-Type Limiting of the Transition Rate in a Superconducting Magnet." Mev.'"

C. B. Fulmer, J. Benveniste, and A. Mitchell, "Proton Scattering by $\mathrm{Ni}^{64}$ and $\mathrm{Zn}^{64}$ at 9.6 and 11.7

J. A. Martin, G. L. Broyles, and J. E. Mann, "Magnetic Field Design for Cyclotron Analogue II."

E. Newman and K. S. Toth, "Nitrogen-Induced Nuclear Reactions in Chlorine."

L. D. Roberts and R. W. Boom, "Studies on a 33-kgauss Superconducting Magnet."

International Conference on Sector-Focused Cyclotrons, Los Angeles, April 16-19, 1962

R. H. Bassel, R. S. Bender, and J. G. Innes, "Analyzing Methods for ORIC Magnetic Fields."

R. J. Jones, "Remarks on Tune-Up of Analogue II."

R. J. Jones, E. D. Hudson, R. S. Livingston, R. S. Lord, M. B. Marshall, W. R. Smith, W. H. White, Jr., and R. E. Worsham, "Unique Features in the Oak Ridge Isochronous Cyclotron."

R. S. Livingston, E. D. Hudson, R. J. Jones, R. S. Lord, J. E. Mann, J. A. Martin, F. M. Russell, N. F. Ziegler, and A. Zucker, "Concepts of an Mc ${ }^{2}$ Cyclotron."

J. A. Martin and J. E. Mann, "Beam Characteristics in Cyclotron Analogue II."

W. H. White, Jr., B. Duelli, and R. J. Jones, "Beam-Phase Measurement Studies."

American Physical Society, Washington, D.C., April 23-26, 1962

R. H. Bassel, R. M. Drisko, E. Rost, and G. R. Satchler, "Analysis of the Inelastic Scattering of Alpha Particles."

C. D. Goodman, J. B. Ball, and C. B. Fulmer, "Interpretations of Low-Lying Levels in Zirconium Isotopes Inferred from $\left(p, p^{\prime}\right),(p, d)$, and $(p, t)$ Spectra."

M. L. Halbert and F. E. Durham, "Angular Momentum Effects in Compound Nucleus Reactions."

E. Newman and K. S. Toth, "Some Single-Nucleon Transfer Reaction Cross Sections Obtained with 27.5-Mev N ${ }^{14}$ Ions." 
K. S. Toth, "The Angular Distribution and Ranges of $\mathrm{N}^{13}$ Particles Resulting from 27.5-Mev N $\mathrm{N}^{14}$ Ions on $\mathrm{Mg}^{24}$."

A. Zucker and B. Linder, "Radiative Capture of $\mathrm{N}^{14}$ on $\mathrm{P}^{31}$.",

E. C. Halbert, R. H. Bassel, and G. R. Satchler, "Optical Model Analysis of Elastic Deuteron Scattering."

High-Energy Instrumentation Conference, Geneva, July 16-18, 1962

R. W. Boom, L. D. Roberts, and R. S. Livingston, "Developments in Superconductor Solenoids." International Symposium on Direct Interactions and Nuclear Reaction Mecbanisms, Padova, Italy, September, 3-8, 1962

J. B. Ball, C. B. Fulmer, and C. D. Goodman, "A Comparison of $(p, d),(p, t)$, and $(p, a)$ Reactions Exciting the Same Final States."

A. Zucker (invited paper), "Reactions in the Nuclear Surface."

Conference on Utilization of Multiparameter Analyzers in Nuclear Physics, Grossingers, Liberty, New York, November 12-15, 1962

D. A. Bromley, C. D. Goodman, and G. D. O'Kelley, "Multi-Parameter Analysis in Accelerator Studies in Nuclear Phy sics."

C. D. Goodman, G. D. O'Kelley, and D. A. Bromley, "Examining and Processing Multiparameter Data from the ORNL-Yale Analyzers."

G. D. O'Kelley, D. A. Bromley, and C. D. Goodman, "The Role of Multiparameter Pulse-Height Analyzers in Radioactivity Studies."

\section{Publications}

Bassel, R. H., R. S. Bender, and J. G. Innes, "Analyzing Methods for ORIC Magnetic Fields," Nucl. Instr. Methods .18-19, 303 (1962).

Bassel, R. H., G. R. Satchler, D. K. McDaniels, and D. L. Hendrie, "Alpha-Gamma Angular Correlations and the Distorted-Waves Theory," Pbys. Letters 1, 295 (1962).

Bassel, R. H., G. R. Satchler, R. M. Drisko, and E. Rost, "Analy sis of the Inelastic Scattering of Alpha Particles. I, Pbys. Rev. 128, 2693 (1962).

Boom, R. W., and R. S. Livingston, "Superconducting Solenoids," IRE Proc. 50, 274-85 (1962).

Boom, R. W., K. S. Toth, and A. Zucker, "Radiation Survey at the LRL 184-Inch Cyclotron," Nucl. Instr. Metbods 18-19, 472 (1962).

Cohen, B. L., "Evaporation Model of Nuclear Reactions," Encyclopaedic Dictionary of Physics 5 , 94-95 (1962).

Drisko, R. M., R. H. Bassel, and G. R. Satchler, "Distorted-Waves Analysis of Quasi-Elastic $(p, n)$ and $\left(\mathrm{He}^{3}, t\right)$ Reactions," Phys. Letters 2, 318 (1962).

Fulmer, C. B., "Elastic Scattering of Protons by Single Isotopes," Phys. Rev. 125, 631-38 (1962).

Gleason, G. I., I. J. Gruverman, and J. L. Need, "Practical Production Yields of Radionuclides with 17- and 21-Mev Protons," Intern. J. Appl. Radiation Isotopes 13, 223-28 (1962). 
Goodman, C. D., J. B. Ball, and C. B. Fulmer, "Single Particle Neutron Bound States in Iron Isotopes and in Cobalt Studied with ( $p, d)$ Reactions," Phys. Rev. 127, 574-82 (1962).

Goodman, C. D., and J. B. Ball, "A System for Detecting and Processing Energy Spectra for Several Types of Charged Particles Simultaneously," pP 163-67 in Proceedings of the Symposium on Nuclear Instruments, Harwell, 1961, ed. by J. B. Birks, Academic Press, New York, 1962.

Goodman, C. D., G. D. O'Kelley, and D.A. Bromley, "A 20,000-Channel Pulse-Height Analyzer with Two Coordinate Address," pp 197-200 in Proceedings of the Symposium on Nuclear Instruments, Harwell, 1961, ed. by J. B. Birks, Academic Press, New York, 1962.

Halbert, M. L., "Surface-Barrier Counters for Nuclear Reaction Studies," pp 403-13 in Proceedings of the Nuclear Electronics Conference, Belgrade, 1961, vol 1, International Atomic Energy Agency, Vienna, 1962.

Harmatz, B., T. H. Handley, and J. W. Mihelich, "Properties of Nuclear Levels in a Number of Odd-A Nuclei $(151 \leq A \leq 191)$," Phys. Rev. 128, 1186-1230 (1962).

Howard, F. T., "Catalogue of Isochronous Cyclotrons," Nucl. Instr. Metbods 18-19, 125 (1962).

Hudson, E. D., R. S. Lord, M. B. Marshall, W. R. Smith, and E. G. Richardson, Jr., "Achievement and Measurement of the ORIC Magnetic Field," Nucl. Instr. Methods 18-19, 159 (1962).

Jones, R. J. "Remarks on Tune-Up of Analogue II," Nucl. Instr. Metbods 18-19, 469 (1962).

Jones, R. J., E. D. Hudson, R. S. Livingston, R. S. Lord, M. B. Marshall, w. R. Smith, W. H. White, Jr., and R. E. Worsham, "Unique Features in the Oak Ridge Isochronous Cyclotron," Nucl. Instr. Methods $18-19,46(1962)$.

Linder, B., and A. Zucker, "Radiative Capture of Nitrogen-14 on Phosphorus-31," Phys. Rev. 127, 1280-83 (1962).

Livingston, R. S., E. D. Hudson, R. J. Jones, R. S. Lord, J. E. Mann, J. A. Martin, F. M. Russell, N. F. Ziegler, and A. Zucker, "Concepts of an Mc ${ }^{2}$ Cyclotron," Nucl. Instr. Methods 18-19, 438 (1962).

Martin, J. A., and J. E. Mann, "Beam Characteristics in Cyclotron Analogue II," Nucl. Instr. Methods 18-19, 461 (1962).

Martin, J. A., J. E. Mann, and R. S. Livingston, "Initial Performance of an Eight-Sector Electron Cyclotron," pP 355-69 in Intemational Conference on Higb Energy Accelerators, Upton, New York, 1961, ed. by M. H. Blewett, Division of Technical Information, AEC, Washington.

Newman, E., "Proton Transfers in the Nitrogen-Boron System at 27.5 Mev," Phys. Rev. 125, 600-605 (1962).

Rost, E., "Analysis of Inelastic Scattering of Alpha Particles-II+," Phys. Rev. 128, 2708 (1962).

Toth, K. S., "Angular Distribution and Ranges of $\mathrm{N}^{13}$ Particles Resulting from 27.5-Mev N ${ }^{14}$ Ions on $\mathrm{Mg}^{24}$," F'bys. Rev. 126, 1489-92 (1962).

White, W. H. Jr., "Magnet Regulators for ORIC," Nucl. Instr. Methods 18-19, 170 (1962).

White, W. H., Jr., B. Duelli, and R. J. Jones, "Beam-Phase Measurement Studies," Nurl. In.str. Methods 18-19, 601 (1962).

Ziegler, N. F., "A High-Voltage Regulator for the ORNL 86-Inch Cyclotron," Nucl. Instr. Metbods 18-19, 197 (1962). 


\section{Advisory Committee}

The Annual Information Meeting of the Electronuclear Division was held jointly with the Physics Division on August 21-22, 1962. The Advisory Committee selected to review the se research programs was as follows:

$\begin{array}{cl}\text { Prof. H. Feshbach } & \begin{array}{l}\text { Massachusetts Institute of Technology } \\ \text { Cambridge } \\ \text { California Institute of Technology } \\ \text { Prof. W. A. Fowler }\end{array} \\ \text { Pasadena } \\ \text { Br. M. Goldhaber } & \begin{array}{l}\text { Brookhaven National Laboratory } \\ \text { Upton, N.Y. }\end{array} \\ \text { Prof. L. J. Rainwater } & \begin{array}{l}\text { Columbia University } \\ \text { New York }\end{array} \\ \text { Prof. J. A. Wheeler } & \begin{array}{l}\text { Princton Univcrsity } \\ \text { Princeton, N.J. }\end{array}\end{array}$

\section{Research Consultants}

University scientists serving, under subcontract, as consultants to the Division include the following:

C. J. Bergeron

H. F. Bowsher

G. Breit

D. A. Bromley

B. L. Cohen

R. M. Drisko

Morton Gordon

Fdith C. Halbert

M. S. Livingston

J. W. Mihelich

H. M. Moseley

J. R. Richardson

H. K. Ticho

A. Timnick

L. D. Wyly, Jr.
Louisian a State University, New Orleans

University of Michigan, Ann Arbor

Yale University, New Haven, Conn.

Yale University, New Haven, Conn.

University of Pittsburgh, Pittsburgh, $\mathrm{Pa}$.

.University of Pittsburgh, Pittsburgh, $\mathrm{Pa}$.

Michigan State University, East Lansing

Massachusetts Institute of Technology, Cambridge

University of Notre Dame, Notre Dame, Ind.

Texas Christian University, Fort Worth

University of California, Los Angeles

University of California, Los Angeles

Michigan State University, East Lansing

Georgia Institute of Technology, Atlanta 


\section{Miscellaneous Activities of the Electronuclear Division}

The Division continues to engage in a variety of educational activities as part of its research function and as a responsibility to the scientific community. Included in these are the employment and training of undergraduate and graduate students, lectures at various universities, seminars, and related matters.

\section{THESIS RESEARCH}

During the past year two PhD candidates completed their thesis research in the Electronuclear Division and were awarded their degrees. G. L. Kochanny, Jr., from Michigan State University did his research problem with the ORNL 86-Inch Cyclotron nuclear physics group. J. W. Wachter, on leave from Union Carbide Nuclear Company (Y-12 Plant), completed his research with the heavy-particle physics group in connection with his studies at the University of Tennessee. Also, M. E. Wetzen and C. B. Hose, both candidates for the master's degree from the University of Tennessee, have worked with the heavy-particle physics group.

\section{OAK RIDGE GRADUATE FELLOWSHIP}

R. F. Sweet, doctoral candidate from the University of Texas, joined the Division in October as an Oak Ridge Graduate Fellow. He is working with the proton physics group on research related to his thesis.

\section{COOPERATIVE RESEARCH PROJECT}

A cooperative program of the Electronuclear Division and Vanderbilt University in the measurement of properties of superconductors at high magnetic. fields was continued through 1962. Both the pulsedmagnet facility at Vanderbilt and the high-field solenoid at ORNL were used for these measurements.

\section{RESEARCH PARTICIPANTS}

Several faculty members from southern universities were at the Laboratory as research participants during the summer of 1962: H. F. Bowsher from the University of Tennessee and F. E. Durham from Tulane University participated in the heavy-particle physics. program. D. H. Rester of Tulane University and J. C. Stoltzfus of Virginia Polytechnic Institute were with the 86-Inch Cyclotron proton physics group. T. I. Hicks from Howard College worked with the ORIC magnet group. C. J. Bergeron from Louisiana State University at New Orleans and R. J. Deck from the Baton Rouge Campus were active in the superconductivity studies. 


\section{VISITING SCIENTISTS}

Two visiting scientists have been on assignments with the isochronous cyclotron group. Bruno Duelli completed his assignment and returned to Kernforschungszentrum, Zyklotron Laboratorium, Karlsruhe, Germany. F. M. Russell received a six-month extension from the National Institute for Research in Nuclear Science, Rutherford High Energy Laboratory, Chilton, England. In an exchange agreement, R. E. Worsham is on assignment to the Rutherford Laboratory.

\section{SUMMER EMPLOYEES}

University students employed for the three summer months included T. H. Boyer, from Yale, and M. P. Fricke, who worked with the high-energy accelerator group; J. C. Huber, from the University of Missouri, and J. D. Jones, from Mississippi State University, assigned to the ORIC group; and C. E. Johnson, from Yale, who worked with the heavy-particle physics group.

\section{CO-OP STUDENTS}

The Division continues to participate in cooperative work-study programs with educational institutions. J. H. Hemman and his alternate, B. W. Jaeger, both from the University of Missouri School of Mines and Metallurgy, completed their work with the ORIC group. R. W. Rutkowski and his alternate, S. Cushner, both from Drexel Institute of Technology, continued their work with the high-energy electronuclear machines group. A new alternating pair from Drexel, T. P. McBride and J. Farrington, began their assignments with the ORIC group.

\section{INVITATION LECTURES}

R. H. Bassel

R. W. Boom
Los Alamos Scientific Laboratory, June 14, 1962

University of Kentucky, December 14, 1962

"Inelastic Scattering of Alpha Particles"

Argonne Users Group, May 11, 1962

Institut fur Strahlen-und Kernphysik der Universitat,

Bonn, Germany, July 23, 1962

"Superconducting Magnets"

Centre d'Etudes Nucleaire, Saclay, France, July 12, 1962

"ORNL Cyclotron Program and Superconducting Magnets"

Federal Institute of Technology (ETH), Zurich, July 20, 1962

"Superconductivity "lests in External Fields and Magnet

Design Problems"

Massachusetts Institute of Technology, December 3, 1962

"ORNL Magnet Program"

Louisiana State University, December 8, 1962

"Superconducting Solenoids and Materials" 
C. D. Goodman

E. E. Gross

M. L. Halbert

J. E. Mann

K. S. Toth

A. Zucker
Georgia Institute of Technology, March 7, 1962

University of Virginia, January 9, 1962

"Studies of Nuclear Structure with Direct Interaction

Proton Reactions"

Nobel Institute, Stockholm, Sweden, August 18, 1962

"Nuclear Structure Studies with 22-Mev Protons"

Louisiana State University, May 3, 1962

"Nuclear Calculation's for the Nuclear Ship 'Savannah",

Gordon Research Conference, Nuclear Chemistry,

New London, N.H., June 28, 1962

"Angular Momentum Effects in Compound Nucleus

Reactions Induced by Heavy Ions"

Vanderbilt University, April 19, 1962

"Necessity for Graphics in Design and Building of

Elcctromechanical Components and Devices"

Gordon Research Conference, Nuclear Chemistry,

New I.ondon, N.H., June 28, 1962.

"Recent Results in Single Nucleon Transfer Reactions"

University of Groningen, Groningen, Holland,

September 12, 1962

"Heavy Ion Induced Nuclear Reactions"

\section{DIVISION RESEARCH SEMINARS}

The Division has sponsored regularly scheduled research seminars on timely topics of special interest to the various programs. The 1962 schedule of seminars, with E. Newman as chairman, included the following:

Fehmiary 13

February 27

March 13

March 20

March 22
F. J. Perey and Rrian Ruck, Neutron Physics

Division, ORNL

"Non-Local Optical Potentials".

Ralph Livingston, Chemistry Division, ORNL

"Paramagnetic Resonance Studies of

Irradiated Solids"

F. M. Russell, National Institute for Research

in Nuclear Science, Rutherford High Energy

Laboratory, Harwell, England, on assignment

with ORNL Electronuclear Division

"Some Topics in Accelerator Research at Harwell"

Seldon Datz, Chemistry Division, ORNL

"Molecular Beam Studies in Physical Chemistry"

H. Maier-Leibnitz, director Laboratorium fur

lechnische Physik der lechnischen Hochschule.

Munchen, Munich, Germany

"Physics Research Using the Swimming Pool

Reactor at Munich, Germany" 
April 3

Maỳ 3

May 8

May 15

May 29

June 5

June 12

July 3

July 10

July 24

July 30

September 4

October 16

December 11

December 18
W. A. Fairbank, Stanford University, Stanford, Calif. "Macroscopic Quantization in Superconductors and Liquid Helium"

H. Bruckmann, Nuclear Physics Institute, University of Bonn, Germany

"Research Program and Cyclotron Developments at the Nuclear Physics Institute, University of Bonn, Germany"

John Landry, Chemical Technology Division, ORNL "Project Plowshare - Peaceful Uses of Nuclear Energy"

K. O. H. Ziock, Yale University, New Haven Conn. "Muonium"

J. R. Richardson, University of California, Los Angeles "Some Recent Operating Results with the UCLA 50-Mev Cyclotron"

Herman Postma, Thermonuclear Division, ORNL

"Recent Experiments on DCX-1"

C. Hwang, University of Minnesota, Minneapolis "Proton Polarization Experiments at Intermediate Energies"

J. A. Martin, Electronuclear Division, ORNL "Beam Characteristics in Cyclotron Analogue II"

L. N. Blumberg, Electronuclear Division, ORNL "Fission Fragment Angular Distributions"

D. C. Peaslee, Australian National University, Canberra "Nuclear E1 Peak Splitting"

K. H. Bhatt, University of Chicago "Single Particle Nilsson Model for Odd- $A$ Nuclei in the 1d 2s Shell"'

H. K. Ticho, University of California, Los Angeles "Strange Particle Resonances"

D. A. Bromley, Yale University, New Haven, Conn.

"Reaction Studies with the Yale LINAC"

C. D. Goodman, Electronuclear Division, ORNL "Comments on Data Handling for Nuclear Reaction Experiments and the Use of the 20,000-Channel Multiparameter Analyzer"

C. J. Bergeron, Louisiana State University, New Orleans

"Longitudinal and Transverse Critical I-H

Characteristics for the Superconductor $\mathrm{Nb}-\mathrm{Zr}$ " 
THIS PAGE

\section{WAS INTENTIONALLY \\ LEFT BLANK}




\author{
ORNL-3432 \\ UC. 34 - Physics \\ TID.4500 (19th ed., Rev.)
}

INTERNAL DISTRIBUTION

1. Biology Library

2-4. Central Research Library

5. Reactor Division Library

6. Laboratory Shift Supervisor

7-8. ORNL - Y-12 Technical Library Document Reference Section

9-58. Laboratory Records Department

59. Laboratory Records, ORNL R.C.

60. J. B. Ball

61. R. H. Bassel

62. R. S. Bender

63. L. N. Blumberg

64. R. W. Boom

65. G. E. Boyd

66. G. L. Broyles

67. C. H. Burbage

68. F. L. Culler

69. H. L. Dickerson

70. B. Duelli

71. C. B. Fulmer

72. C. A. Gault

73. C. D. Goodman

74. E. E. Gross

75. M. L. Halbert

76. B. Harmatz

77. C. S. Harrill

78. A. Hollaender

79. A. S. Householder

80. F. T. Howard

81. E. D. Hudson

82. R. W. Johnson.

83. R. J. Jones

84. W. H. Jordan

85. M. T. Kelley

86. J. A. Lane

87. C. E. Larson

88. T. A. Lincoln

89. S. C. Lind

90. R. S. Livingston
91. R. S. Lord

92. J. E. Mann

93. M. B. Marshall

94. J. A. Martin

95. F. E. McDaniel

96. K. Z. Morgan

97. S. W. Mosko

98. M. L. Nelson

99. E. Newman

100. E. L. Olson

101. G. A. Palmer

102. J. J. Pinajian

103. R. F. Pruitt

104. A. E. Pugh

105. E. G. Richardson, Jr.

106. A. W. Riikola

107. F. M. Russell

108. J. A. Russell

109. L. B. Schneider

110. E. D. Shipley

111. M. J. Skinner

112. W. R. Smith

113. A. H. Snell

114. J. A. Swartout

115. E. H. Taylor

116. K. S. Toth

117. C. L. Viar

118. A. M. Weinberg

119. W. H. White, Jr.

120. R. E. Worsham

121. N. F. Ziegler

122-123. A. Zucker

124. D. L. Judd (consultant)

125. B. L. Cohen (consultant)

126. M. Deutsch (consultant)

127. A. M. Clogston (consultant)

128. L. J. Rainwater (consultant)

129. J. A. Wheeler (consultant) 


\section{EXTERNAL DISTRIBUTION}

130. R. W. McNamee, Union Carbide Corporation, New York

131. Research and Development Division, AEC, ORO

132-768. Given distribution as shown.in TID-4500 (19th ed., Rev.) under Physics category ( 75 copies - OTS) 
Progress reports previously issued by the Electronuclear Division are listed below with their periodending dates.

\begin{tabular}{ll} 
ORNL-1173 & September 30, 1951, Part I \\
ORNL-1235 & September 30, 1951, Part II \\
ORNL-1269 & December 31, 1951, Part I \\
ORNL-1270 & December 31, 1951, Part II \\
ORNL-1315 & March 31, 1952, Part II \\
ORNL-1339 & March 31, 1952, Part I \\
ORNL-1345 & June 30, 1952, Part I \\
ORNL-1346 & June 30, 1952, Part II \\
ORNL-1383 & September 30, 1952 \\
ORNL-1531 & March 20, 1953 \\
ORNL-1663 & September 20, 1953 \\
ORNL-1670 & March 20, 1954 \\
ORNL-1795 & September 20, 1954 \\
ORNL-1884 & March 20, 1955 \\
ORNL-2030 & September 20, 1955 \\
ORNL-2139 & March 20, 1956 \\
ORNL-2140 & March 20, 1956 \\
ORNL-2434 & October 1, 1957 \\
ORNL-2740 & October 1, 1958 \\
ORNL-3047 & March 1, 1960 \\
ORNL-3083 & January 16, 1961 \\
ORNL-3257 & December 31, 1961 \\
\hline
\end{tabular}

